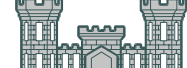

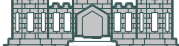

U.S. Army Corps of Engineers

Portland District

\title{
Water Velocity Measurement on an Extended-Length Submerged Bar Screen at John Day Dam
}

FINAL REPORT

April 2001

Contract DE-AC06-76RLO1830 Related Services

MPIR W66QKZ00482760

Pacific Northwest National Laboratory

P.O. Box 999

K6-85

Richland, Washington 99352 


\title{
DISCLAIMER
}

This report was prepared as an account of work sponsored by an agency of the United States Government. Neither the United States Government nor any agency thereof, nor Battelle Memorial Institute, nor any of their employees, makes any warranty, express or implied, or assumes any legal liability or responsibility for the accuracy, completeness, or usefulness of any information, apparatus, product, or process disclosed, or represents that its use would not infringe privately owned rights. Reference herein to any specific commercial product, process, or service by trade name, trademark, manufacturer, or otherwise does not necessarily constitute or imply its endorsement, recommendation, or favoring by the United States Government or any agency thereof, or Battelle Memorial Institute. The views and opinions of authors expressed herein do not necessarily state or reflect those of the United States Government or any agency thereof.

\author{
PACIFIC NORTHWEST NATIONAL LABORATORY \\ operated by \\ BATTELLE \\ for the \\ UNITED STATES DEPARTMENT OF ENERGY \\ under Contract DE-AC06-76RLO1830
}




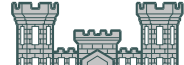

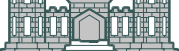

U.S. Army Corps of Engineers

Portland District

\section{Water Velocity Measurement on an Extended-Length Submerged Bar Screen at John Day Dam}

\section{FINAL REPORT}

Prepared by:

M.A. Weiland

(Pacific Northwest National Laboratory)

C.W. Escher

(MEVATEC Corporation)

April 2001

Contract DE-AC06-76RLO-1830 Related Services

MIPR W66QKZ00482760

Pacific Northwest National Laboratory

P.O. Box 999

K6-85

Richland, Washington 99352 


\section{Executive Summary}

This report describes a study of water velocity around extended-length submerged bar screen (ESBSs) at John Day Dam. The study was conducted for the U.S. Army Corps of Engineers by AScI Corporation and MEVATEC Corporation in March of 2000. This report was prepared by Pacific Northwest National Laboratory.

Testing and modifications to the perforated plates of the prototype ESBSs have been ongoing since the screens were installed at John Day Dam in 1996. After testing of the prototype ESBSs, the perforated plates that attach to the backs of the screens were found to have bolt failures and weld cracks. Several bolt and stiffener modifications were made, but cracking of the stiffener bars continued to be a problem. Following a series of vibration tests and modal analysis on many perforated plate configurations, smaller perforated plates were installed. Testing of the modified ESBSs showed no structural problems and minimal plate vibration. However, during testing of the modified ESBSs with the smaller plates, higher fish mortality was observed. Physical model testing of the new system at the Waterways Experiment Station (WES) showed significant changes to the flow from the original design. Less water was flowing through the perforated plates, more water was flowing up the screens at an increased velocity.

The objective of the research described in this report was to collect water velocity measurements near the surface of the bar screens on the ESBSs using an acoustic Doppler velocimeter and to compare those results with physical model data from WES.

Velocity measurements for comparison with physical model data were collected on March 24 and 25, 2000, using two acoustic Doppler velocimeters near the surface of the bar screens of a prototype ESBS at John Day Dam. Measurements were collected 6.75 in. above the surface of the bar screens along the bottom $36 \mathrm{ft}$ of the ESBS. Most data were collected at $155 \mathrm{MW}(21,000 \mathrm{cfs})$. A truncated data set of the bottom $6.4 \mathrm{ft}$ of the screen at $138 \mathrm{MW}(18,600 \mathrm{cfs})$ was also collected. Measurements were collected over the surface of the bar screens using a modified sweep bar to traverse the ADV's the length and width of the bar screens. This allowed us to collect velocity measurements near the surface of $66 \%$ of the bar screens on the ESBSs.

Results show that, at $155 \mathrm{MW}$ velocities were highest at the bottom of the ESBS $(7.7 \mathrm{ft} / \mathrm{sec})$ and declined to the lowest velocities about one-fourth of the distance up the ESBS. Flow velocities increased again to $7.7 \mathrm{ft} / \mathrm{sec}$ about $4 \mathrm{ft}$ below the top of the bar screen, which was as far up as we could collect data with our sampling method. Above this point, according to physical model data, velocities continued to increase to over $10 \mathrm{ft} / \mathrm{sec}$. The region of low flow was an area of transition where flows switched to moving up instead of down the bar screens. The actual point of flow change was $6.4 \mathrm{ft}$ above the bottom of the bar screens.

Velocity measurements collected on the ESBSs at John Day Dam were on average $0.8 \mathrm{ft} / \mathrm{sec}$ higher than physical model results. There is not enough information available to determine if the higher velocities at the ESBSs and if contact with the bar screens were the causes of increased fish injury and mortality rates. Another possible location of injury is in the gate well. Physical model data show flows in the gate well greater than $14 \mathrm{ft} / \mathrm{sec}$. These high velocities and possible turbulence in the gate well may result in fish injury and mortality from shear forces and contact with the side walls of the gate well. 


\section{Acknowledgments}

Many people made valuable contributions to this study. Rich Vaughn, Portland District USACE, designed and oversaw the construction of the modified sweep bar and positioning electronics and spent many long hours in the field making this project happen. Craig Smith, MEVATEC Corp., helped with construction of the modified sweep bar and helped with figures for the report. Kyle Bouchard, MEVATEC Corp., created tables and cleaned up figures for the report. Thanks also go to Derrek Faber, USGS-BRD Columbia River Research Laboratory, for helpful hints with Tecplot; Vadim Polonichko, Applications Engineer at SonTek, for providing assistance with programs to unwrap aliased data; Andy Goodwin, Civil \& Environmental Engineering, Cornell University, for helped with MATLAB; and Tony Wahl, U.S. Bureau of Reclamation, for making changes to WinADV to suit our needs. A special thanks goes to the rigging crew and staff at John Day Dam for their invaluable support and assistance. 


\section{Contents}

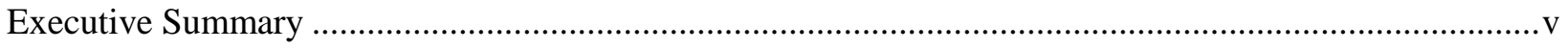

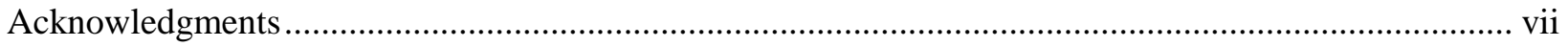

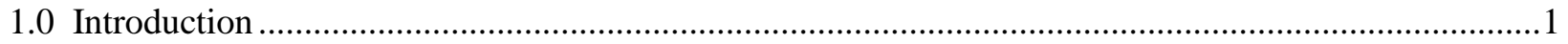

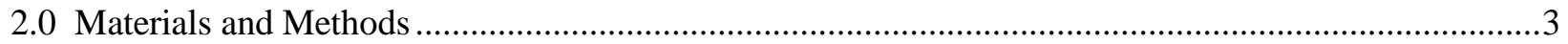

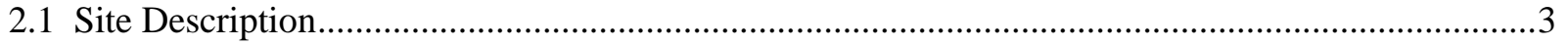

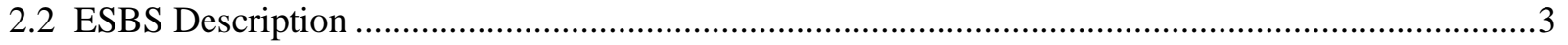

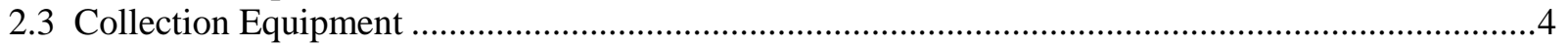

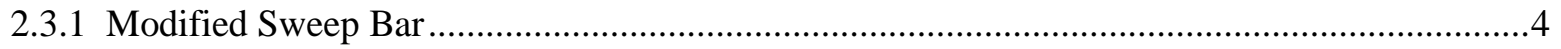

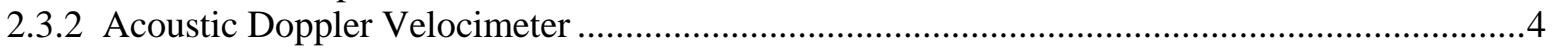

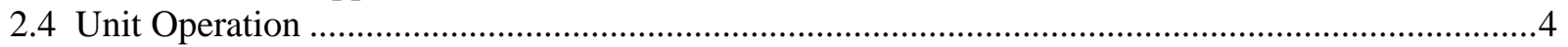

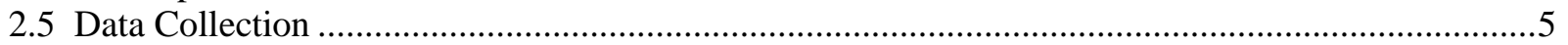

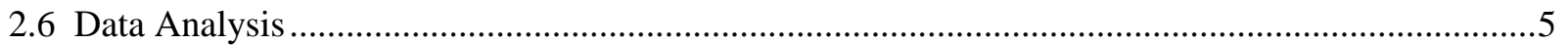

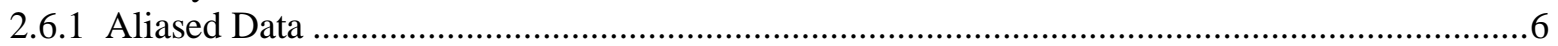

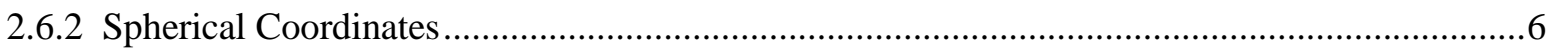

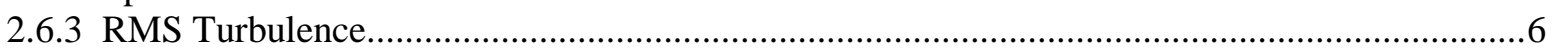

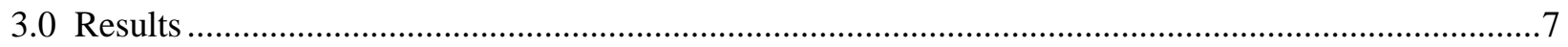

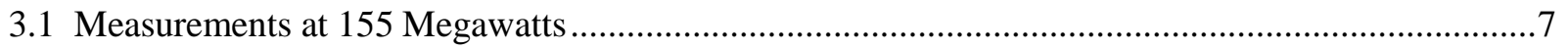

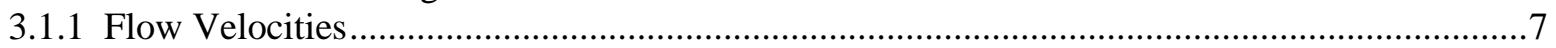

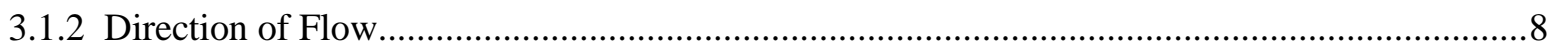

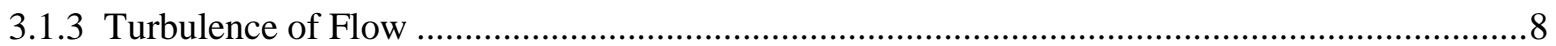

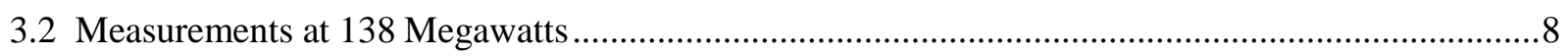

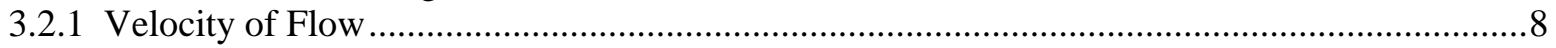

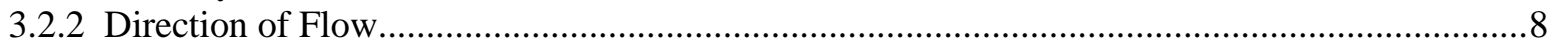

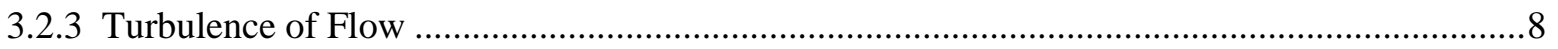

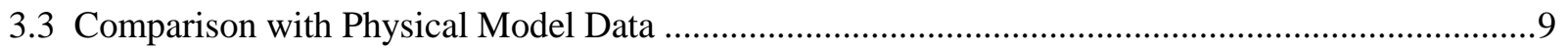

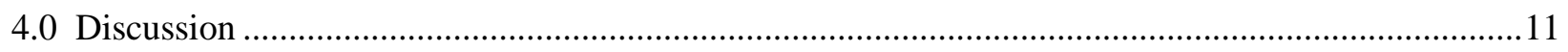

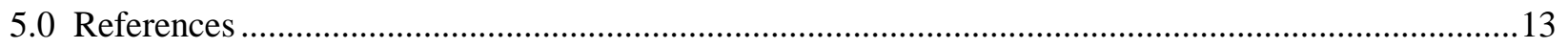

Appendix A - X, Y, and Z Vector Velocities at 155 MW Turbine Load ........................................... A-1

Appendix B_Spherical Coordinates for Velocity Data_at 155 MW Turbine Load .............................. A-15

Appendix C - Turbulence Intensity Values at 155 MW Turbine Load .............................................. A-29

Appendix D - X, Y, and Z Vector Velocities at 138 MW Turbine Load ............................................. A-43

Appendix E - Spherical Coordinates for Flow Data at 138 MW Turbine Load................................... A-47

Appendix F _ Turbulence Intensity Values at 138 MW Turbine Load ............................................. A-51 


\section{Figures}

Figure 1. Side view of a turbine intake with an ESBS deployed in the gate well slot in the extended position

Figure 2. ESBS being moved by the gantry crane at John Day Dam.

Figure 3. View of a bar screen, used to guide fish, on the upstream face of the ESBS showing position of the modified sweep bar and ADV relative to the bar screens Fig-3

Figure 4. View of the modified sweep bar, attached to the drive chains, in position across the bar screens on the ESBS .Fig-4

Figure 5. The stepper motor, attached to the end of the threaded shaft, was housed in a waterproof canister

Figure 6. A Tecnadyne position sensor was mounted on the threaded shaft, at the opposite end from the stepper motor to provide data on the position of the ADVs on the sweep Bar as the location flow data was being collected along the horizontal (Y) axis on the bar screens of the ESBS

Figure 7. A Tecnadyne position sensor was mounted to the end of the drive shaft at the top of the ESBS

Figure 8. The control panel used to control and monitor movement of the ADVs across the bar screens Fig-8

Figure 9. View of the modified sweep bar during construction showing a cable carrier Fig-9

Figure 10. Diagram of the head of an ADV describing the transmission and receiving of signals and location of the sample volume relative to the ADV Fig-10

Figure 11. View of the ESBS showing locations where flow data were collected with ADVs Fig-11

Figure 12. Relation between Cartesian and spherical coordinate systems Fig-12

Figure 13. Contour plot of magnitude velocity ( $\mathrm{ft} / \mathrm{sec}$ ) $6.75 \mathrm{in}$. from the surface of the bar screens on the ESBS at 155-MW turbine load Fig-13

Figure 14. Magnitude water velocity ( $\mathrm{ft} / \mathrm{sec})$ from the lower end $(0 \mathrm{ft})$ to $36 \mathrm{ft}$ up the ESBS Fig-14

Figure 15. Vector water velocity $(\mathrm{ft} / \mathrm{sec}) 6.75 \mathrm{in}$. from the surface of the bar screens on the ESBS at 155-MW turbine load Fig-15

Figure 16. The face view of the ESBS shows vector water velocities $(\mathrm{ft} / \mathrm{sec})$ in the $\mathrm{Y} / \mathrm{Z}$ plane, $6.75 \mathrm{in}$. from the surface of the bar screens, in four vertical zones at $2.67 \mathrm{ft}(\mathrm{A})$, $7.33 \mathrm{ft}(\mathrm{B}), 12.67 \mathrm{ft}(\mathrm{C})$, and $17.33 \mathrm{ft}(\mathrm{D})$ across the ESBS. 
Figure 17. Vector water velocity $(\mathrm{ft} / \mathrm{sec}$ ) in the $\mathrm{X}$-axis (perpendicular to the bar screens) from the lower end $(0 \mathrm{ft})$ to $36 \mathrm{ft}$ up the ESBS

Figure 18. Vector water velocity $(\mathrm{ft} / \mathrm{sec})$ in the $\mathrm{Y}$-axis (horizontal to the bar screens) from the lower end $(0 \mathrm{ft})$ to $36 \mathrm{ft}$ up the ESBS.

Figure 19. Vector water velocity ( $\mathrm{ft} / \mathrm{sec}$ ) in the Z-axis (longitudinal to the bar screens) from the lower end $(0 \mathrm{ft})$ to $36 \mathrm{ft}$ up the ESBS Fig-19

Figure 20. Angular direction of flow Phi $(\phi)$ in the perpendicular plane (X-axis) to the bar screens at 155-MW turbine load

Figure 21. Angular direction of flow Theta $(\theta)$ in the horizontal plane (Y-axis) to the bar screens at 155-MW turbine load

Figure 22. Contour plot of magnitude flow RMS turbulence 6.75 in. from the surface of the bar screens on the ESBS at $155 \mathrm{MW}$ turbine load Fig-22

Figure 23. Contour plot of RMS turbulence into the screen (x-axis) $6.75 \mathrm{in}$. from the surface of the bar screens on the ESBS at 155-MW turbine load

Figure 24. Contour plot of RMS turbulence across the screen (y-axis) $6.75 \mathrm{in.}$ from the surface of the bar screens on the ESBS at 155-MW turbine load

Figure 25. Contour plot of RMS turbulence longitudinal to the screen (z-axis) 6.75 in. from the surface of the bar screens on the ESBS at 155-MW turbine load

Figure 26. Contour plot of magnitude velocity ( $\mathrm{ft} / \mathrm{sec}) 6.75 \mathrm{in}$. from the surface of the bar screens on the ESBS at 138-MW turbine load

Figure 27. Vector water velocity $(\mathrm{ft} / \mathrm{sec}) 6.75 \mathrm{in}$. from the surface of the bar screens on the ESBS at $138 \mathrm{MW}$ turbine load

Figure 28. The face view of the ESBS shows vector water velocities $(\mathrm{ft} / \mathrm{sec})$ in the $\mathrm{Y} / \mathrm{Z}$ plane, $6.75 \mathrm{in}$. from the surface of the bar screens, in four vertical zones at $2.67 \mathrm{ft}(\mathrm{A})$, $7.33 \mathrm{ft}(\mathrm{B}), 12.67 \mathrm{ft}(\mathrm{C})$, and $17.33 \mathrm{ft}(\mathrm{D})$ across the ESBS Fig-28

Figure 29. Contour plot of magnitude flow RMS turbulence 6.75 in. from the surface of the bar screens on the ESBS at 138-MW turbine load

Figure 30. Contour plot of RMS turbulence into the screen (x-axis) 6.75 in. from the surface of the bar screens on the ESBS at 138-MW turbine load

Figure 31. Contour plot of RMS turbulence across the screen (y-axis) $6.75 \mathrm{in}$. from the surface of the bar screens on the ESBS at 138-MW turbine load

Figure 32. Contour plot of RMS turbulence longitudinal to the screen (z-axis) 6.75 in. from the surface of the bar screens on the ESBS at 138-MW turbine load Fig-32 
Figure 33. Comparison of velocity measurements between data collected in the field 6.75 in. from the bar screens at 21,000 cfs, and physical model data collected at scale 4 and 8 in. from the screen at 20,800 cfs, longitudinally (z-axis) on the ESBS ...Fig-33 


\subsection{Introduction}

John Day Dam is a multipurpose U.S. Army Corps of Engineers project located on the Columbia River at river mile 216. In 1996 three prototype extended-length submerged bar screens (ESBSs) were delivered to John Day Dam for testing. The ESBSs are guidance structures installed in the turbine intakes to divert fish out of the turbine intakes into the gate well and through juvenile fish bypass channels. Submerged traveling screens (STS) are currently used at John Day Dam to route fish away from the turbine intakes. ESBSs were found to improve guidance efficiency and cause less descaling of fish than the STS at other dams (McComas et al. 1993) so ESBSs are being evaluated at John Day Dam.

Prototype testing and modifications to the ESBSs have been ongoing since installation in 1996. After testing, the prototype ESBSs were found to have bolt failures and weld cracks on the perforated plates, which attach to the downstream side of the screens. Several bolt and stiffener modifications were made, but cracking of the stiffener bars continued to be a problem. Following a series of vibration tests and modal analysis on many perforated plate configurations, smaller plates were installed. After testing of the smaller plates on the ESBSs, no structural problems were found and plate vibration was acceptably low (USACE 1999).

During testing of the modified ESBSs with the smaller plates, higher fish mortality was observed. Model testing of the new system at the Waterways Experiment Station (WES) showed significant changes to the flow from the original design (USACE 1999).

The objectives of this research project were 1) to collect water velocity data near the surface of the ESBSs at $138 \mathrm{MW}(18,600 \mathrm{cfs})$ and $155 \mathrm{MW}(21,000 \mathrm{cfs})$ load levels using an acoustic Doppler velocimeter (ADV) and 2) to compare the results with model data from WES. 


\subsection{Materials and Methods}

\subsection{Site Description}

John Day Dam is a multipurpose U.S. Army Corps of Engineers project located on the Columbia River at river mile 216. The dam is composed of a powerhouse, spillway, and navigation lock, with fish facilities at either end of the dam. The powerhouse is $1,975 \mathrm{ft}$ long and consists of 16 turbines with nameplate capacity of $135 \mathrm{MW}$ each and overload capacity of $155.3 \mathrm{MW}$ each.

\subsection{ESBS Description}

In 1996 three prototype ESBSs were delivered to John Day Dam for testing. The ESBSs are guidance structures installed to aid in guiding fish up and out of the turbine intakes and into the gate well and juvenile fish bypass channels. The ESBSs are lowered into the gate slots of the turbine intake and the inner frame is pivoted upstream to a 55-degree angle and held in place by a support frame (Figure 1).

The ESBS is approximately $53 \mathrm{ft}$ long by $23 \mathrm{ft}$ wide, spanning the width of the turbine intake (Figure 2). It weighs about 114,000 lbs. The ESBS consists of a main outer frame that provides the structural framework for all the subcomponents of the ESBS system and supports all forces imposed during turbine operation (USCE 1999). The inner frame assembly is $40 \mathrm{ft}$ long by $20 \mathrm{ft}$ wide. The upstream surface of the inner frame consists of 1/8-in. wedge wire bar screen with $1 / 8$-in. spaces in between (Figure 3 ). This surface diverts the fish upward into the gate well while allowing water to pass through the turbine intake. The downstream surface of the inner frame is composed of perforated plates used to control water flow through the bar screen.

A sweep bar that spans the width of the bar screen is mounted on the upstream surface of the inner frame and moves vertically along the bar screens removing debris, which could clog the screen and injure the fish (Figure 4). The sweep bar consists of a bristle brush mounted on a steel tube. The brush assembly is mounted on two drive chains that pull the sweep bar up and down the vertical face of the screen. There are sprockets at the upper and lower ends of the screen to drive the chains that run vertically along the screen. The bottom sprockets act as idlers while the sprockets at the top of the screen are driven by a drive shaft. One end of the drive shaft connects to a speed reducer/motor assembly while the other end connects to a control box (USACE 1999).

The brush control system provides the operating limits of the sweep bar and controls the cycle time of the sweeping operation. A proximity switch mounted near the end of the drive shaft monitors magnets on a wheel mounted on the end of the drive shaft. The proximity switch counts the rotations of the wheel, monitors the location of the sweep bar, and controls the stopping position of the sweep bar at the top and bottom of the screen. 


\subsection{Collection Equipment}

\subsubsection{Modified Sweep Bar}

A modified sweep bar was built for mounting of the acoustic Doppler velocimeters (ADVs) and to provide horizontal and vertical movement of the ADV across the bar screens. The modified sweep bar was mounted to the ESBS on the same drive chains as were used by the sweep bar and was moved vertically using the ESBS control system in manual mode. Instead of a steel tube, a piece of 12-in. by 3in. channel was used for the main structure of the modified sweep bar (Figure 4). Two 10-ft pieces of 1.25-in. threaded shaft were mounted in blocks of ultra high molecular weight (UHMW) plastic in the channel as a screw to move the ADV along the channel. A Pacific Scientific stepper motor sealed in a waterproof housing was attached to one end of the shaft to turn the shafts (Figure 5). The housing was maintained at external pressure while underwater by an air line tapped into the housing, and fed by an air compressor. A Tecnadyne model 194 rotary position sensor was mounted to the other end of the shaft to monitor shaft rotation and determine the position of the ADV on the modified sweep bar (Figure 6).

A second Tecnadyne rotary position sensor was mounted to the end of the drive shaft at the top of the screen to determine the vertical position of the modified sweep bar (Figure 7). Calibrated output from both position sensors was monitored with Durant Eclipse series quadrature meters in a control panel located at deck level (Figure 8). A Pacific Control 5645 Indexer Drive used to operate the stepper motor was also installed in the control panel. From the control panel the horizontal position of the ADV on the bar screens was controlled and position was known to within several hundredths of an inch.

ADV cables were installed in cable carriers and attached within the channel to provide guidance and prevent damage to the cables as the ADV traversed the modified sweep bar (Figure 9). A 1/8-inch aluminum plate covered the cables and about $80 \%$ of the channel piece. Two UHMW plates were positioned at either end of the aluminum plate and attached to the threaded shaft with machined 1.25inbrass nuts. The ADVs were clamped into aluminum blocks attached to the UHMW plates (Figure 3). The two ADVField ${ }^{\mathrm{TM}}$ processor underwater canisters containing the signal processing hardware for the ADVs were attached to plates on the lower edge of the modified sweep bar (Figure 3).

\subsubsection{Acoustic Doppler Velocimeter}

Water velocity measurements were collected using two Sontek $5 \mathrm{MHz}$ ADVOcean Probe ${ }^{\mathrm{TM}}$ acoustic Doppler velocimeters and two Sontek ADVField ${ }^{\mathrm{TM}}$ processor underwater canisters. The ADV transmits with one acoustic transmitter and receives on three acoustic receivers. The transmitter is located in the center and the receivers are mounted on the end of the signal-conditioning module. The sampling volume of the probe is approximately $0.12 \mathrm{in.}$ and is located about $7 \mathrm{in}$. from the acoustic transmitter (Figure 10). The ADV measures the water velocity using the Doppler effect. The transmitter emits a short pulse of known frequency into the water. A portion of the acoustic energy is reflected off particulate matter in the water back to the receivers at a different frequency. This frequency difference (Doppler shift) is proportional to the particle velocity, which is assumed to be moving at the same velocity as the water. The ADV probe was referenced with the $\mathrm{x}$-axis perpendicular to the screen, the $\mathrm{y}$-axis across the screen, and the $\mathrm{z}$-axis longitudinal to the screen.

\subsection{Unit Operation}

Unit 7 was used for testing the ESBS. The ESBS with the modified sweep bar and ADVs was deployed in the C-slot. During testing, forebay elevation was $264.6 \mathrm{ft}$ with a $102.9 \mathrm{ft}$ head differential and the forebay water temperature was $45^{\circ} \mathrm{F}$. Velocity measurements were collected while the unit operated at 
$155 \mathrm{MW}(21,000 \mathrm{cfs})$. A second truncated data set was collected with unit operation at $138 \mathrm{MW}(18,600$ cfs). To account for equivalent head loss due to installation of the ESBS, heads of $100.6 \mathrm{ft}$ and $100.0 \mathrm{ft}$ were used for the 155-MW and 138-MW loads, respectively, to calculate discharge. The unit was held at fixed load and head differential varied little during the test.

\subsection{Data Collection}

Water velocity measurements were collected about 6.75 in. above the surface of the bar screens from the lower tip ( $0 \mathrm{ft}$ ) to approximately $36 \mathrm{ft}$ up the bar screens. Measurements were collected at predetermined fixed locations on the screen for two-minute intervals. Data were also collected during the moves between fixed-point locations. Moves between locations usually lasted between 30 and 60 seconds. Data from each of the move measurements was divided into five equal samples and the data was averaged for each sample (Figure 11). Samples were collected at $25 \mathrm{~Hz}$.

\subsection{Data Analysis}

Data were filtered to remove outlying and erroneous values. If the signal-to-noise ratio was less than 15 or the correlation between data within a data point was less than $70 \%$, the values were omitted. (The ADV pings 250 times per second. We collected data at $25 \mathrm{~Hz}, 25$ data points per second; therefore, 10 pings are averaged to provide a data point. Correlation is estimated between these 10 pings.) A spike filter was also used to remove spikes in the data, usually caused by large particles in the water, and where any value greater than $1 \mathrm{~g}$ was omitted. The 1-g filter was selected after comparing several different filter settings.

The spike filter used was developed for the $\mathrm{WinADV}^{\mathrm{TM}}$ analysis package where

given three adjacent velocity measurements at sample numbers $n-1, n$, and $n+1$ :

$$
\left(\mathrm{V}_{\mathrm{x}}, \mathrm{V}_{\mathrm{y}}, \mathrm{V}_{\mathrm{z}}\right)_{\mathrm{n}-1}\left(\mathrm{~V}_{\mathrm{x}}, \mathrm{V}_{\mathrm{y}}, \mathrm{V}_{\mathrm{z}}\right)_{\mathrm{n}}\left(\mathrm{V}_{\mathrm{x}}, \mathrm{V}_{\mathrm{y}}, \mathrm{V}_{\mathrm{z}}\right)_{\mathrm{n}+1}
$$

For each component the two accelerations were computed:

$$
\begin{aligned}
& A_{1 x}=\frac{\left(X_{x, n}-V_{X, n-1}\right)}{\Delta t} \\
& A_{2 x}=\frac{\left(X_{x, n+1}-V_{X, n}\right)}{\Delta t}
\end{aligned}
$$

The difference between the two values is the change in acceleration $\Delta \mathrm{A}_{\mathrm{x}}$ from sample $\mathrm{n}-1$ to sample $\mathrm{n}+1$. The iteration is then repeated for the $\mathrm{y}$ and $\mathrm{z}$ velocity components. The acceleration components are then combined to compute a scalar change in acceleration:

$$
\Delta A=\sqrt{\Delta A_{x}^{2}+\Delta A_{y}^{2}+\Delta A_{z}^{2}}
$$

Data sets were divided into two groups: the data that were collected at fixed locations and data that were averaged over the move between fixed locations. Data were averaged for the entire data set at the fixed locations and were averaged into five evenly spaced samples across the move between fixed locations. 


\subsubsection{Aliased Data}

Data near the upper and lower tips of the screen were aliased where water velocities were higher than the upper limit recording levels of the ADV, causing a phase reversal in the data and erroneous values. This occurred when the measured phase difference between the two acoustic pulses transmitted and received by the ADV exceeded $180^{\circ}$. The data were corrected using the "unwrap" function in MATLAB ${ }^{\mathrm{TM}}$. This function unwraps radian phases $(\mathrm{P})$ by changing absolute jumps greater than $\pi$ to their $2 \pi$ complement, unwrapping along the first non-singleton dimension of $\mathrm{P}$.

\subsubsection{Spherical Coordinates}

A spherical coordinate system was used to define the direction of the bulk flow. To define the spherical coordinates we used a polar axis and a perpendicular equatorial plane. Originating at the intersection of the plane and the axis, the origin $(\mathrm{O})$, a ray to point $\mathrm{P}$ is constructed with a length of rho $(\rho)$, the magnitude velocity. The angle phi $(\phi)$ is the angle between the line $\mathrm{OP}$ and the positive polar axis and theta $(\theta)$ is the angle between the initial ray and the projection of OP to the equatorial plane (Figure 12), where

$$
\begin{aligned}
& \text { Rho }=\sqrt{x^{2}+y^{2}+z^{2}} \\
& \text { Phi }=\cos ^{-1}(z / r h o) \\
& \text { Theta }=\tan ^{-1}(y / x)
\end{aligned}
$$

In reference to the bar screens, the $\mathrm{x}, \mathrm{y}$, and $\mathrm{z}$ coordinates are the same orientation as was used for the $\mathrm{ADV}$, where positive $\mathrm{x}$ is flow perpendicular to the bar screens, positive $\mathrm{y}$ is horizontal flow parallel to the bar screens, and positive $\mathrm{z}$ is longitudinal flow up the bar screens. From an upstream perspective looking downstream, $X$-vector velocities are perpendicular to the screen with positive $X$ into the screen, $\mathrm{Y}$-vector velocities are across the short axis of the screen with positive $\mathrm{Y}$ toward the right, and Z-vector velocities are along the long axis of the screen with positive $\mathrm{Z}$ up the screen. Theta is positive when flow is moving toward the right, across the screen, and negative when flow is moving to the left. If theta is zero, flow is moving longitudinal to the screen. Phi is always positive. When phi is $90^{\circ}$, the flow is perpendicular to the screen. When phi is greater than $90^{\circ}$, the direction of flow is down the screen. When phi is less than $90^{\circ}$, the direction of flow is up the screen. If phi is $0^{\circ}$ the bulk flow would be parallel to the screen and no flow would be through the screens.

\subsubsection{RMS Turbulence}

The root mean square (RMS) of the water velocity was calculated for each of the four fixed sample locations across the screen. The root mean square of the water velocity was also averaged for data points sampled across the spaces moved between each of the fixed sample locations.

$$
R M S\left[V_{i}\right]=\sqrt{\frac{\sum V_{i}^{2}-\left(\sum V_{i}\right)^{2} / n}{n-1}} \mathbf{V}_{\mathbf{i}} \quad \text { where } \mathbf{i}=\mathbf{x}, \mathbf{y} \text {, or } \mathbf{z} .
$$




\subsection{Results}

Water velocity measurements were collected along the upstream face of the ESBS in the C-Slot of Unit 7 at John Day Dam on March 24 and 25, 2000. Velocity measurements at 155 MW (21,000 cfs) were collected from the lower tip of the bar screen (0.0), to about $36 \mathrm{ft}$ from the bottom. Forebay elevation was $264.6 \mathrm{ft}$ above mean sea level and the head differential was $102.9 \mathrm{ft}$. The water temperature was $45^{\circ} \mathrm{F}$.

A second set of velocity measurements was recorded at $138 \mathrm{MW}$ (18,600 cfs). However, measurements were only collected over the bottom $6.5 \mathrm{ft}$ of the bar screen due to a mechanical failure resulting in termination of testing.

\subsection{Measurements at 155 Megawatts}

\subsubsection{Flow Velocities}

From an upstream perspective looking downstream, $X$-vector velocities are perpendicular to the screen with positive $\mathrm{X}$ into the screen, $\mathrm{Y}$-vector velocities are across the short axis of the screen with positive $\mathrm{Y}$ toward the right, and $\mathrm{Z}$-vector velocities are along the long axis of the screen with positive $\mathrm{Z}$ up the screen (Figure 12).

Velocities were highest near the lower and upper ends of the screen with velocities up to $7.75 \mathrm{ft} / \mathrm{sec}$. The lowest velocities were about $2.2 \mathrm{ft} / \mathrm{sec}$, approximately one-fourth of the distance up the screen. Velocities increased above this point reaching highs again at the top of the screen (Figure 13 and 14).

Flows were not directed up or down the screens over the entire length of the ESBS. At the transition zone between positive and negative flows in the Z-axis, where velocities were the lowest, the flow was across the screen toward the right side, and toward the left on the far left side of the screen (Figure 15 and 16). This was due to the reversal in flow direction. Streamlines on the left side of the screen were quicker to orient in the $+Z$ direction as flow moved up the screen than on the right side. Downward flows did not orient in the $-\mathrm{Z}$ before the water passed under the lower end of the screen.

There was a significant difference in velocity between the left and right sides of the screens (ANOVA $\mathrm{P}<0.05$ ) with flows being highest on the right side. The mean flow on the right side of the ESBSs was on average $0.2 \mathrm{ft} / \mathrm{sec}$ higher than on the left side of the bar screens.

The vector velocity into the bar screen (x-axis) declined steadily from the bottom to the top of the screen Near the top of the screen, $35.89 \mathrm{ft}$, almost no flow passed through the screen. This rapid change from about $2.5 \mathrm{ft} / \mathrm{sec}$ to less than $0.5 \mathrm{ft} / \mathrm{sec}$ in the $\mathrm{x}$-axis occurred over a distance change of less than $1 \mathrm{ft}$ on the screen (Figure 17).

There was larger variation in the Y-vector velocities across the screen (y-axis) than either the X- or Zvector velocities (Figure 18). This variation was due to differences in flow direction and velocity of flow between the right and left sides of the ESBS. Also, if the ADVs were not lined up exactly with the screen offset, it would be most noticeable in this direction.

The vector velocities along the length of the screen (z-axis) increased over the entire length of the screen (Figure 19) with negative flow around the bottom of the screen and flows increasing in the positive direction up the screen. Values for all $\mathrm{X}, \mathrm{Y}$, and $\mathrm{Z}$ velocity vectors are provided in Appendix $\mathrm{A}$. The magnitudes of velocities are provided in Appendix B with the spherical coordinate data. 


\subsubsection{Direction of Flow}

Spherical coordinates were calculated from the velocity components to provide a three-dimensional analysis of the flow. Theta is positive when flow is moving toward the right across the screen and negative when flow is moving to the left. If theta is zero, flow is moving longitudinal to the screen. Phi is always positive. When phi is $90^{\circ}$ the flow is perpendicular to the screen. When phi is greater than $90^{\circ}$ the direction of flow is down the screen and when phi is less then $90^{\circ}$ the direction of flow is up the screen.

Phi showed downward flows through the screen for the bottom $6.5 \mathrm{ft}$ of the screen. Above this point, flows were oriented up and through the screen. At $\mathrm{Z}=35.89 \mathrm{ft}$, the screen flows were almost longitudinal to the screen with very little of the flow passing through (Figure 20). As indicated by the Y vectors of the flow, theta showed similar flow across the screen to the right with flows becoming a little less skewed toward the top of the screen (Figure 21).

\subsubsection{Turbulence of Flow}

The root mean square (RMS) turbulence of the velocity showed greatest turbulence near the lower end of the screen and in the area of transition between downward and upward flows (Figure 22). There was no significant difference in turbulence between the left and right sides of the screen (ANOVA $P>0.05$ ). Flow was more turbulent in the $\mathrm{X}$ and $\mathrm{Y}$ vectors (Figures 23 and 24) than in the $\mathrm{Z}$ vector (Figure 25). Magnitude and vector turbulence values are provided in Appendix C.

\subsection{Measurements at 138 Megawatts}

\subsubsection{Velocity of Flow}

Velocities were greater than $6.5 \mathrm{ft} / \mathrm{sec}$ at the lower end of the screen and declined to about $2.5 \mathrm{ft} / \mathrm{sec}$ where flows changed direction, $6.4 \mathrm{ft}$ above the bottom of the screen (Figure 26). Flow at the bottom of the screen angled toward the right side of the screen and began to orient up the screen at $6.4 \mathrm{ft}$ above the bottom of the screen, the point where data collection was terminated (Figure 27 and 28). Values for all X, $\mathrm{Y}$, and $\mathrm{Z}$ velocities are provided in Appendix D and the magnitude velocities with the spherical coordinate data are provided in Appendix E.

\subsubsection{Direction of Flow}

The longitudinal component of the flow, phi, showed downward flows through the screen up to $6.4 \mathrm{ft}$ above the bottom where flows started to move up the screen. At this location there was still downward movement of water on the left side of the screen but about one-third of the way across the screen flows were beginning to orient up the screen.

\subsubsection{Turbulence of Flow}

The RMS turbulence of the magnitude velocity showed greatest turbulence in the lower left corner and at two patches about $1.6 \mathrm{ft}$ from the base of the screen. At the screen tip where flows were highest the turbulence was lowest, except at the lower left corner (Figure 29). Flow was more turbulent in the $X$ and $\mathrm{Y}$ vectors (Figures 30 and 31) than in the $\mathrm{Z}$ vector (Figure 32). This was also true at $155 \mathrm{MW}$. Magnitude and vector turbulence values are provided in Appendix F. 


\subsection{Comparison with Physical Model Data}

Velocity measurements collected on the 1:12 scale physical model at WES were measured, to scale, 4 in. and 8 in. from the physical model bar screens at a scale discharge of 20,800 cfs. ADV measurements collected in the field were $6.75 \mathrm{in}$. from the bar screens and discharge was $21,000 \mathrm{cfs}$. The flow velocities collected $6.75 \mathrm{in}$. from the bar screens in the field were significantly higher (ANOVA P $<0.05$ ) than the physical model data for both 4-in. and 8-in. distances (Figure 33). Field-measured velocities averaged 0.8 $\mathrm{ft} / \mathrm{sec}$ higher than either of the physical model tests. Though field measurements were higher than both physical model outputs, the physical model and field values did follow a similar trend. 


\subsection{Discussion}

ADVs were used to collect water velocity data near the bar screens on the ESBS at John Day Dam and successfully provided fine-scale three-dimensional velocity data profiles over a majority of the screen. From the setup used it was not possible to collect measurements closer than $2.67 \mathrm{ft}$ from either edge of the bar screen or over the upper $4 \mathrm{ft}$ of the bar screens. Measurements could be collected on over $66 \%$ of the bar screen surfaces on the ESBSs.

The high sampling rate and small sample volume of the ADV provided an excellent tool to collect velocity measurements close to the structure. The velocities of the flows were within the bounds of the $\mathrm{ADV}$. The $\mathrm{X}$ and $\mathrm{Y}$-axes of the probe measured flow without aliasing to over $8 \mathrm{ft} / \mathrm{sec}$. The $\mathrm{Z}$-axis measured flows to about $2.5 \mathrm{ft} / \mathrm{sec}$ before aliasing. Although the aliased data can be unwrapped, orienting the probe with the Z-axis oriented across the ESBS in the direction of low flows could alleviate this problem.

Velocity measurements collected along the bar screens of the ESBS at John Day Dam were higher (approximately $0.8 \mathrm{ft} / \mathrm{sec}$ ) than measurements collected in the WES physical model. The conditions tested in the field were slightly different than the simulated conditions tested in the physical model but probably not enough to see a $0.8 \mathrm{ft} / \mathrm{sec}$ average difference. The discharge of our test $(21,000 \mathrm{cfs})$ was 200 cfs higher than the physical model test $(20,800 \mathrm{cfs})$. This difference probably was not great enough to explain the difference in the results. Differences in distance from the screen could account for differences in velocity, but our data were collected between the two distances sampled on the physical model and resulting flows were higher than both of the physical model tests. Our results suggest that the physical model estimates are lower than in situ measurements.

There is not enough information available to determine if the increased velocities encountered by fish at the bar screens and contact with the bar screens increased injury and mortality rates. The highest rates of impingement and contact with the bar screens occur in the middle section of the ESBS (Nestler and Davidson 1995). This is where flows were measured to be nearly perpendicular to the screen. Also, as a result of the bar screens efficiency to pass flow with negligible head loss, the bar screens may be hard to detect by fish, which may lead to strikes and impingement of juvenile salmonids (Nestler and Davidson 1995).

Another possible location of injury is in the gate well. Physical model data show that flows in the gate well can be greater than $14 \mathrm{ft} / \mathrm{sec}$ in some areas. These high velocities and possible turbulence in the gate well may result in fish contact with the concrete walls of the gate well causing fish injury and mortality. 


\subsection{References}

McComas, R.L., D.A. Brege, W.D. Muir, B.P. Sandford, and D.B. Dey. 1993. Studies to Determine the Effectiveness of Extended-Length Submersible Bar Screens at McNary Dam, 1992. Coastal Zone and Estuarine Studies Division, National Marine Fisheries Service, Seattle, Washington.

Nestler, J. and R. Davidson. 1995. Imaging Smolt Behavior on an Extended-Length Submerged Bar screen and an Extended-Length Submerged Traveling Screen at the Dalles Dam in 1993. Prepared for U.S. Army Corps of Engineers District, Portland, Oregon.

U.S. Army Corps of Engineers Walla Walla District. 1999. Lower Snake and Columbia Rivers Extended Length Submerged Bar Screen (ESBS) System-Wide, Letter Report, 90 Percent Draft Report. 
PNNL-13517

Figures 


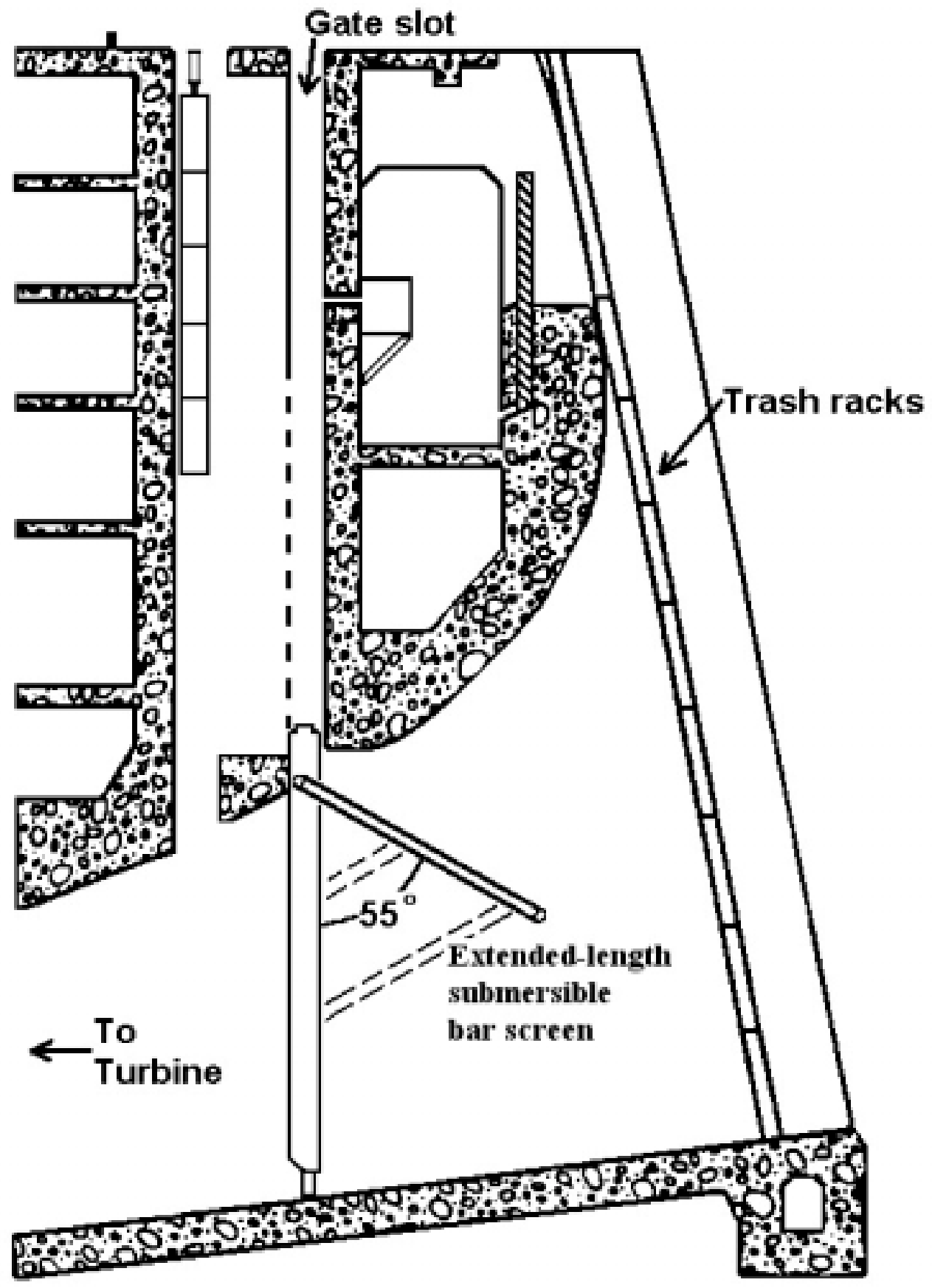

Figure 1. Side View of a Turbine Intake with an ESBS Deployed in the Gate Well Slot in the Extended Position 


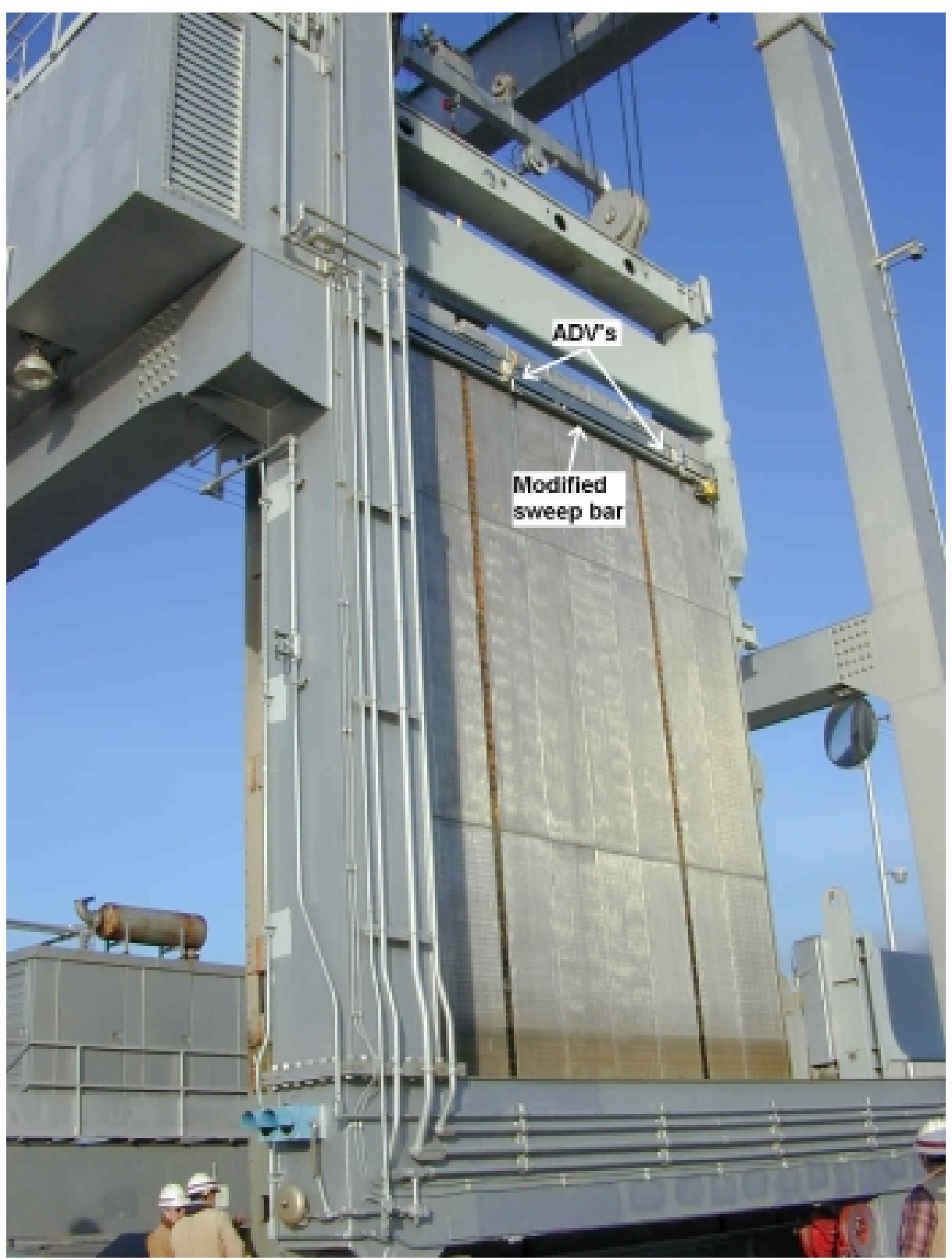

Figure 2. ESBS Being Moved by the Gantry Crane at John Day Dam. The modified sweep bar with the ADVs attached at the uppermost position at which measurements could by taken on the bar screens. 


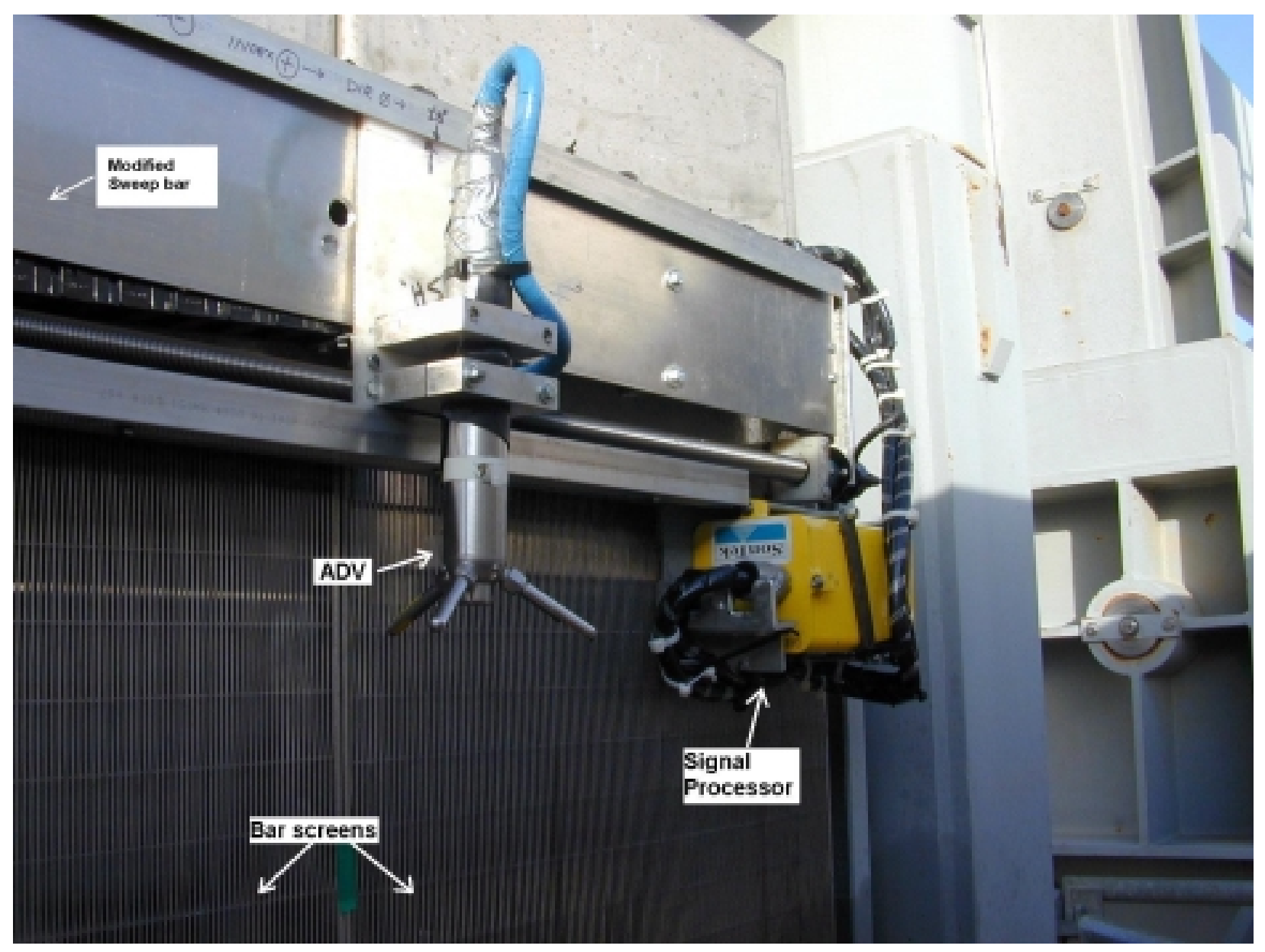

Figure 3. View of a Bar Screen, Used to Guide Fish, on the Upstream Face of the ESBS Showing Position of the Modified Sweep Bar and ADV Relative to the Bar Screens. Also in the figure are one of the two ADV's, $6.75 \mathrm{in}$. from the surface of the bar screen, and an underwater canister containing the signal processing hardware. 


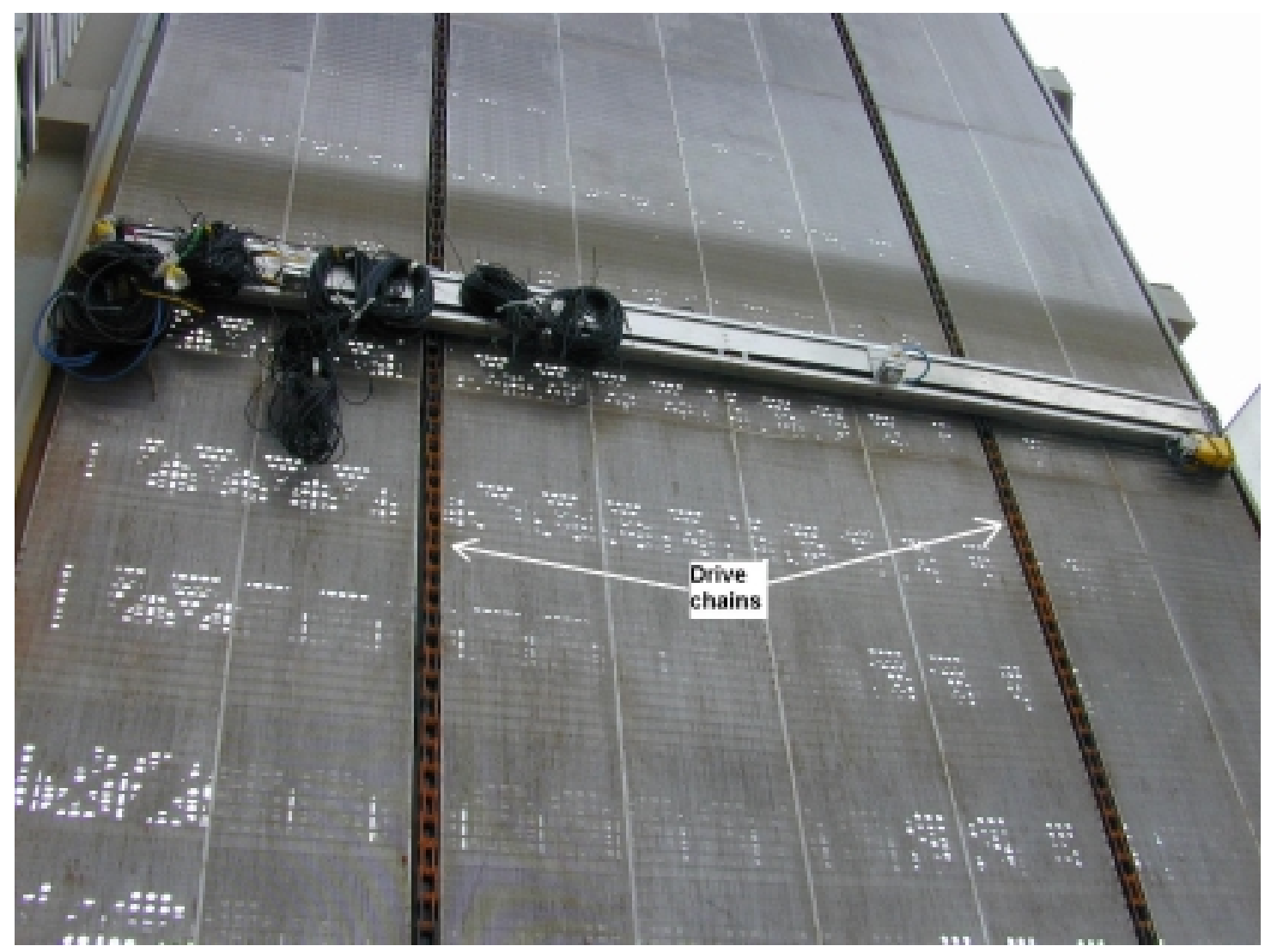

Figure 4. View of the Modified Sweep Bar, Attached to the Drive Chains, in Position across the Bar Screens on the ESBS. No brushes were attached to the modified sweep bar to reduce restriction of flow up the bar screens. 


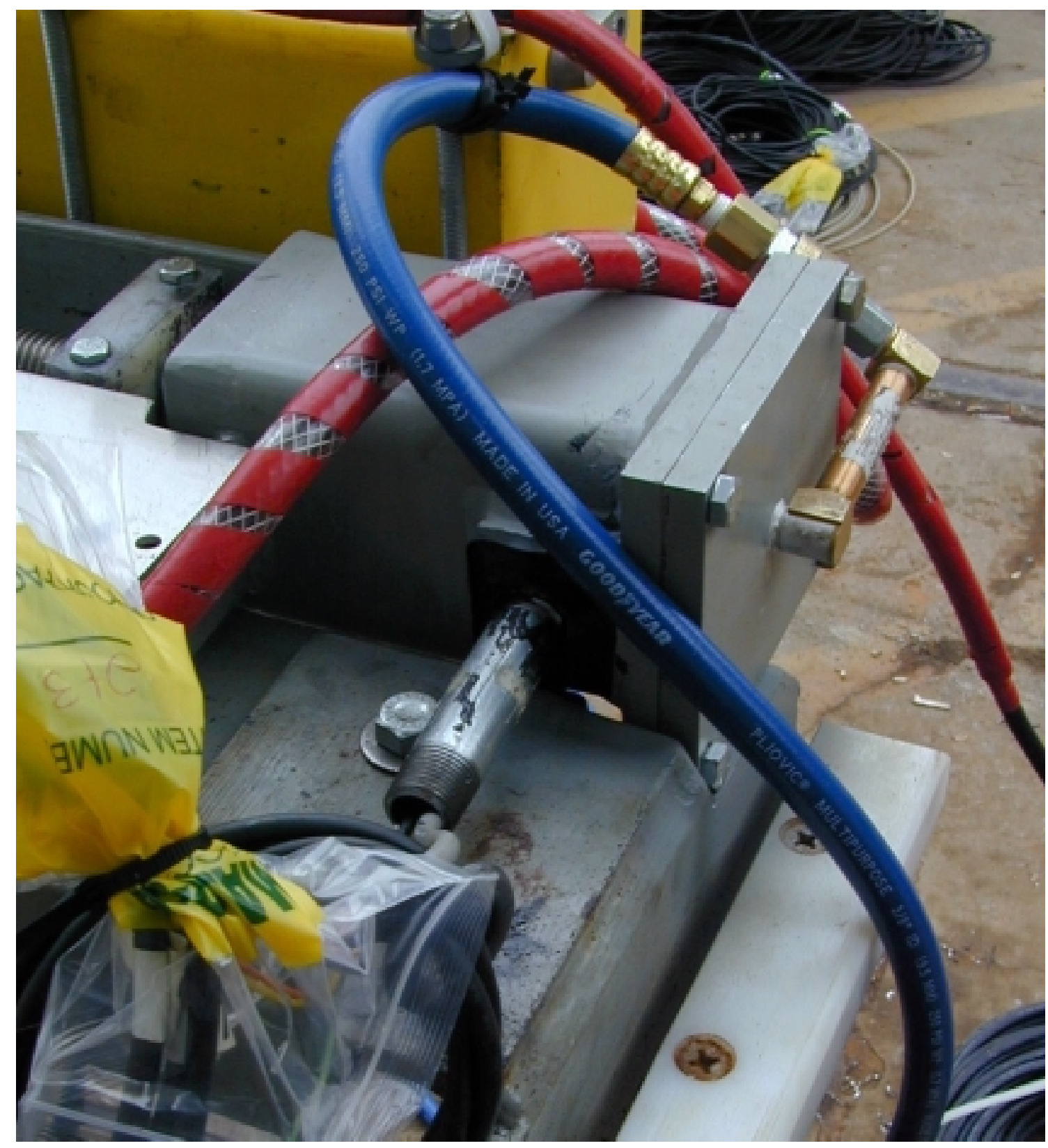

Figure 5. The Stepper Motor, Attached to the End of the Threaded Shaft, Was Housed in a Waterproof Canister. An air line was threaded into the end of the housing to keep the inside of the housing at equal pressure with the outside environment in order to reduce the chance of seal leakage. 


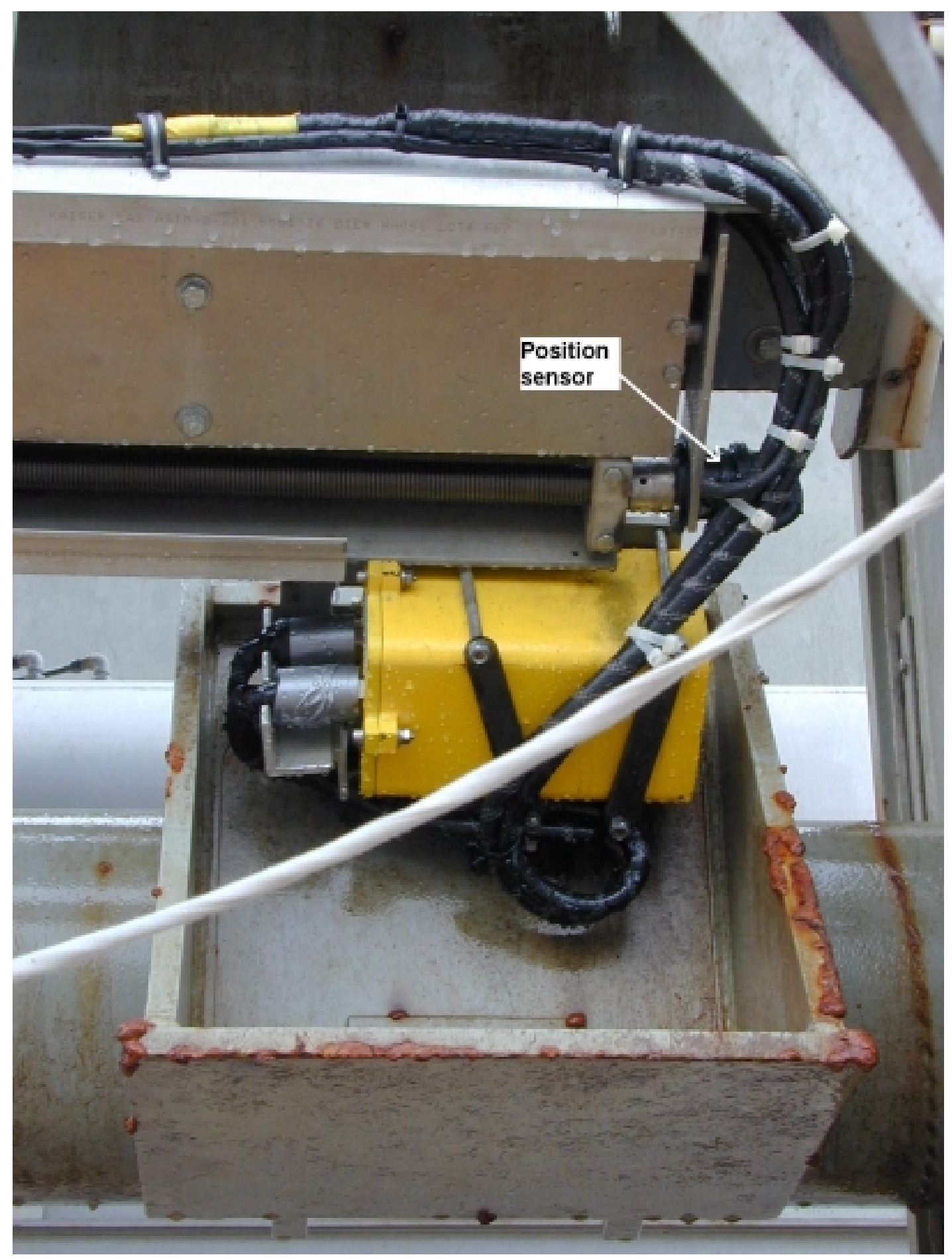

Figure 6. A Tecnadyne Position Sensor was Mounted on the Threaded Shaft at the Opposite End from the Stepper Motor to Provide Data on the Position of the ADVs on the Sweep Bar as the Location Flow Data Was Collected along the Horizontal (Y) Axis on the Bar Screens of the ESBS 


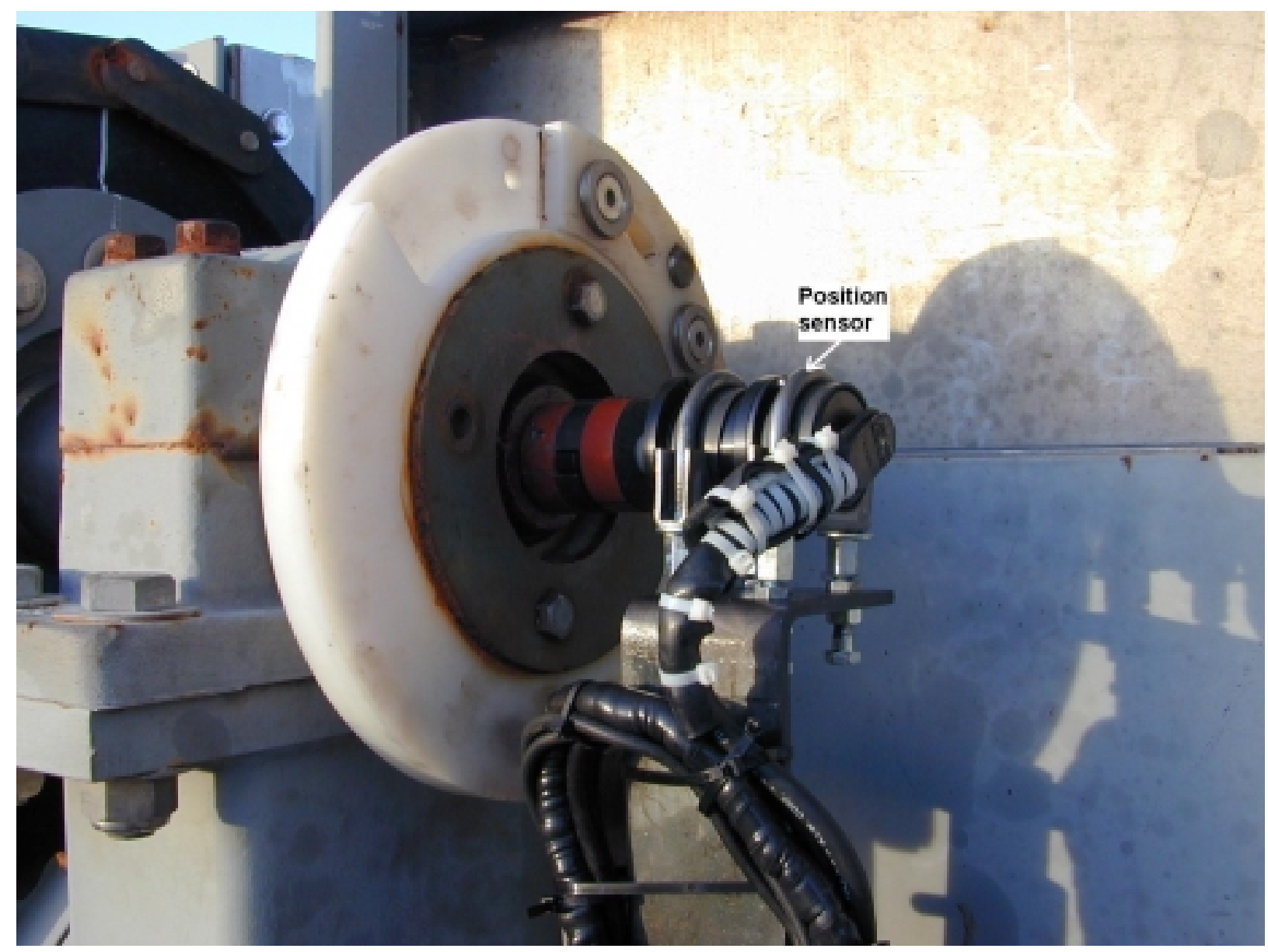

Figure 7. A Tecnadyne Position Sensor Was Mounted to the End of the Drive Shaft at the Top of the ESBS. The drive shaft is connected to the chains that move the sweep bar up and down the face of the ESBS. The position sensor provided vertical position data on the modified sweep bar. 


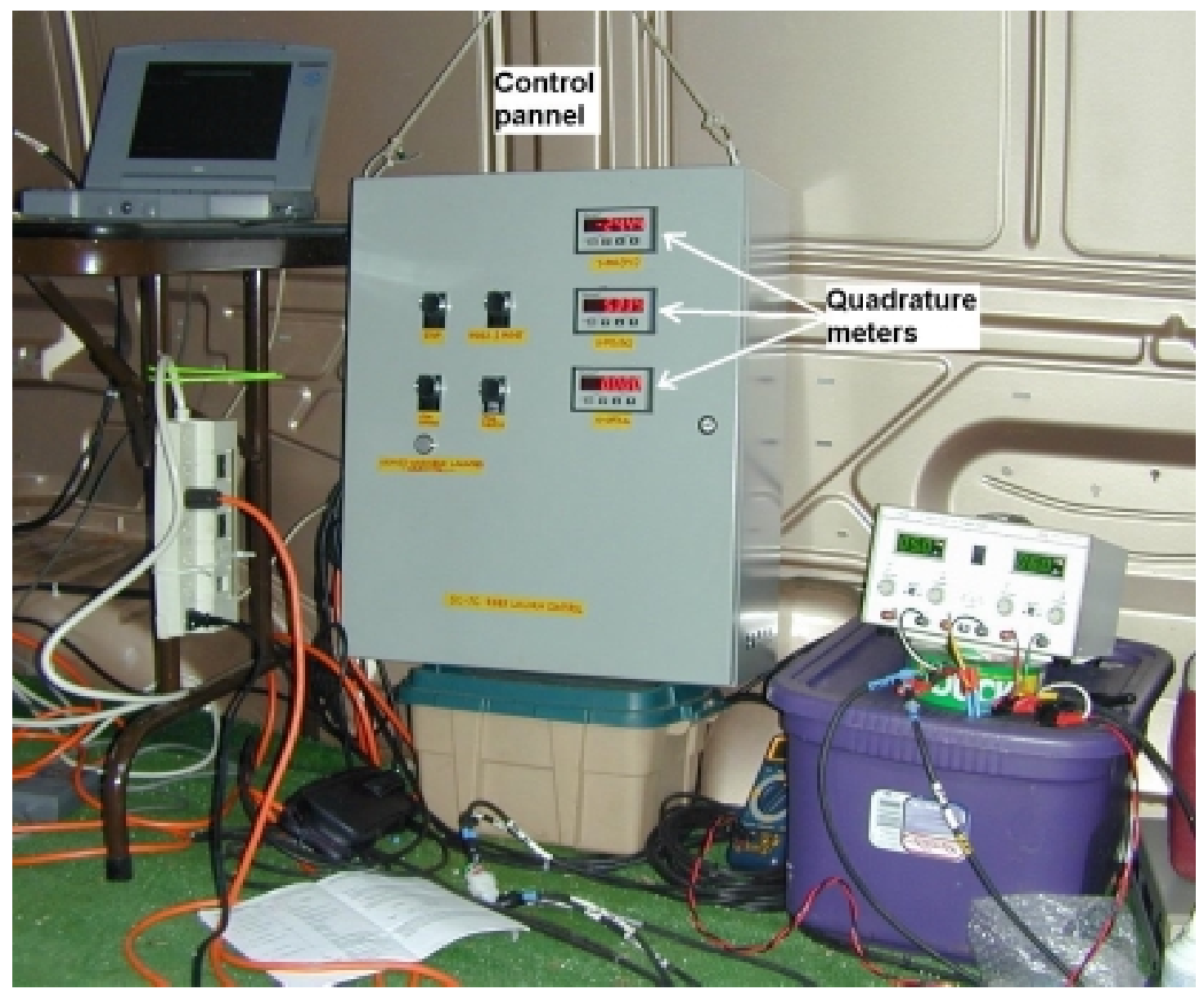

Figure 8. The Control Panel Used to Control and Monitor Movement of the ADVs across the Bar Screens. The quadrature meters displayed position of the modified sweep bar on the bar screens. These data were supplied by the two Tecnadyne position sensors. 


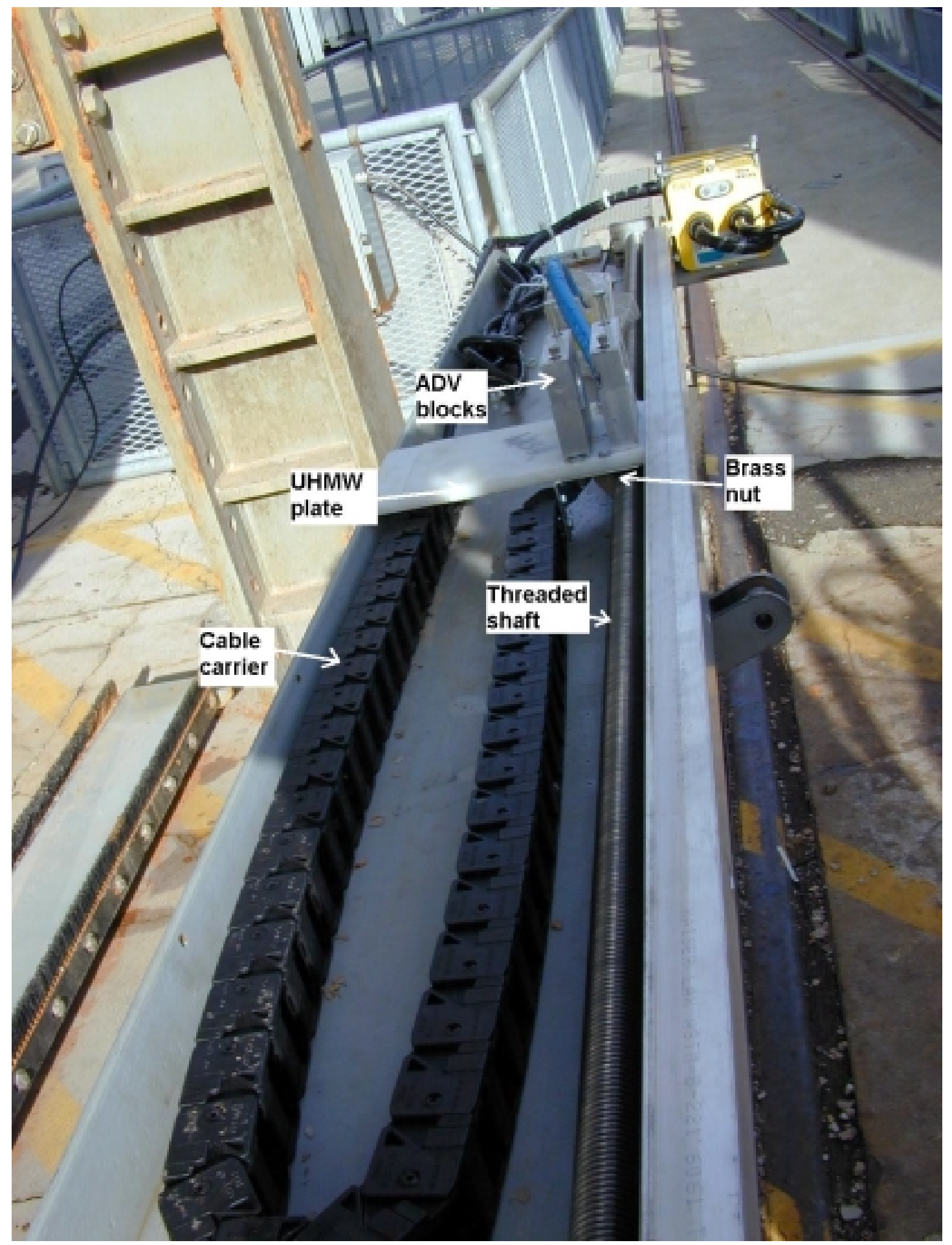

Figure 9. View of the Modified Sweep Bar during Construction showing a Cable Carrier. Also shown are the aluminum blocks that held the ADVs. These blocks were attached to a UHMW plastic plate that was connected to a brass nut that was threaded onto the shaft. 


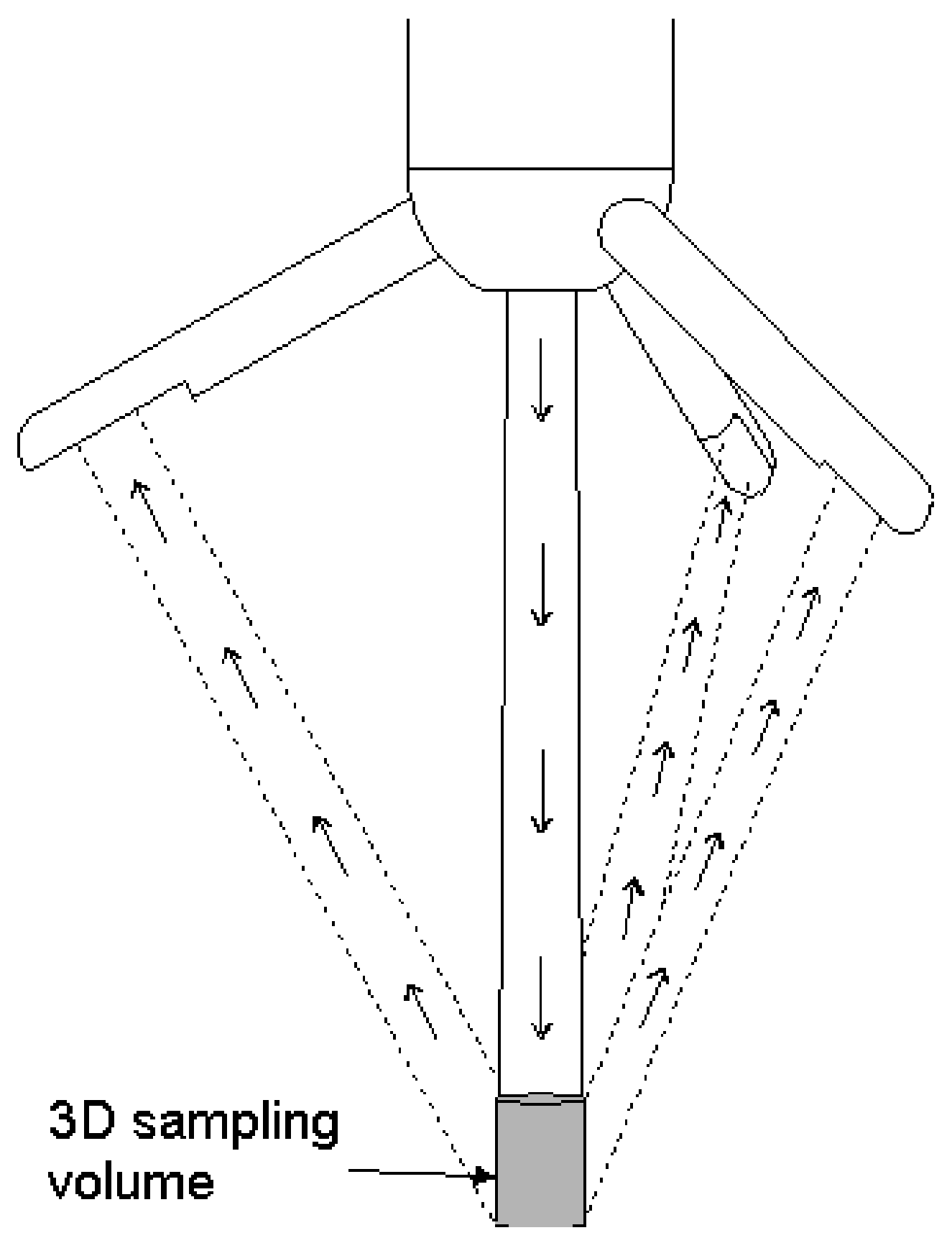

Figure 10. Diagram of the Head of an ADV Describing the Transmission and Receiving of Signals and Location of the Sample Volume Relative to the ADV 


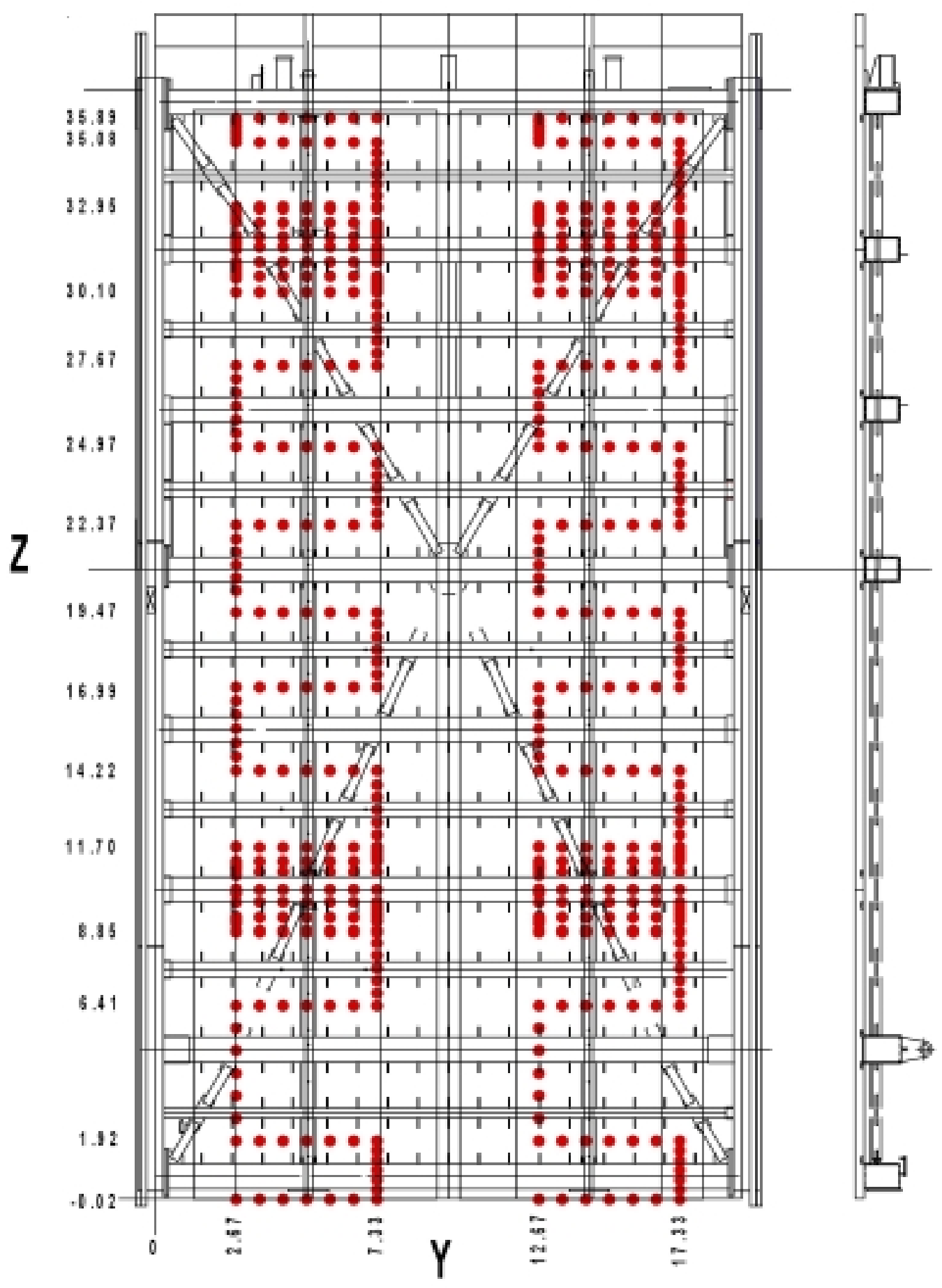

Figure 11. View of ESBS Showing Locations where Flow Data Were Collected with ADVs. Stationary samples were collected at $2.67,7.33,12.67$, and $17.33 \mathrm{ft}$ on the horizontal axis and at each of the corresponding vertical positions ( $\mathrm{ft}$ ) indicated on left side of diagram. Flow measurements were also collected during the move between stationary positions. These measurements were divided into five equal sizes and averaged, providing five estimates across the move. 

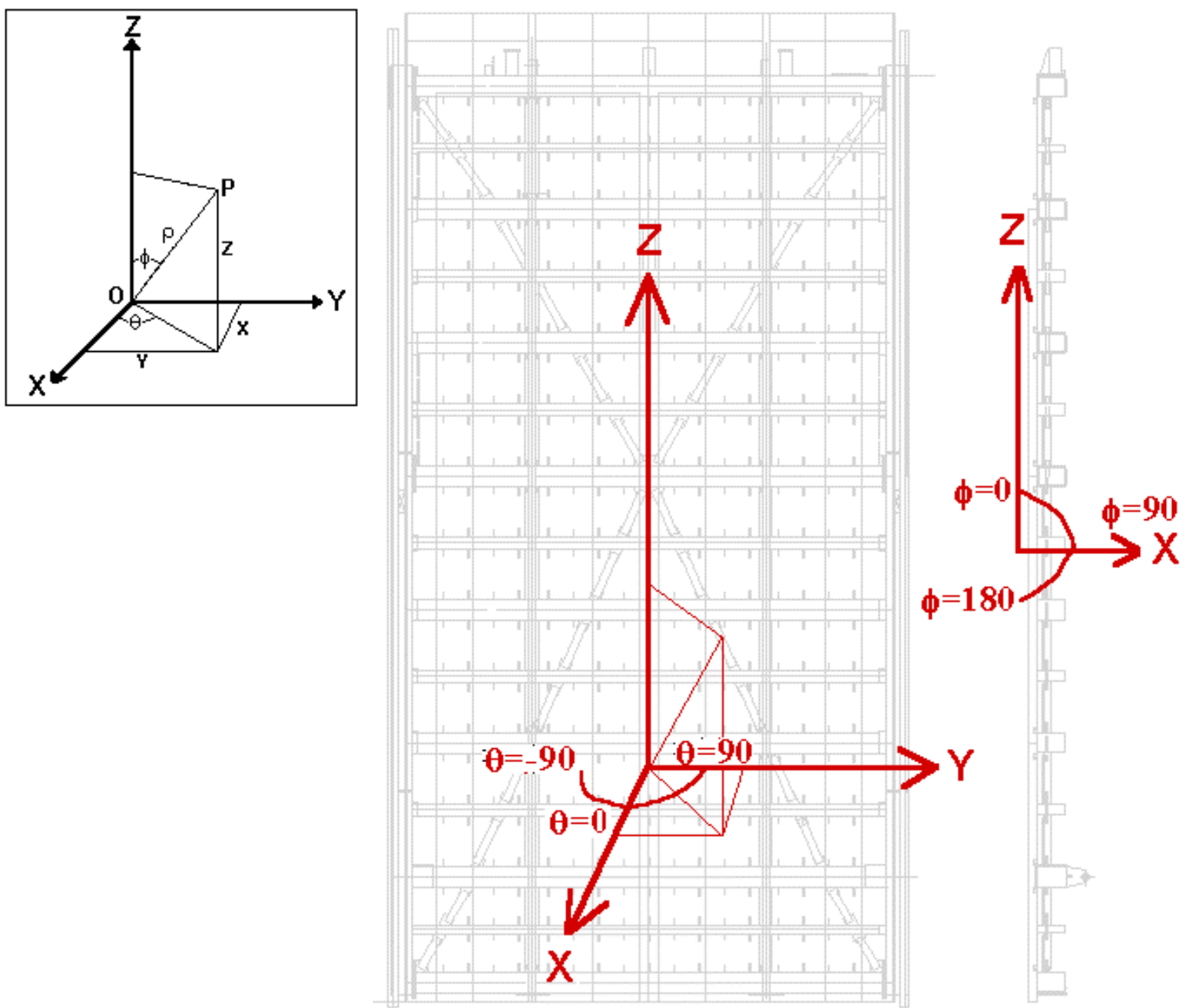

Figure 12. Relationship between Cartesian and Spherical Coordinate Systems. In the Cartesian system $\mathrm{X}$-axis was perpendicular, Y-axis horizontal (across the ESBS), and Z-axis longitudinal (up the ESBS) to the bar screens. In the spherical coordinate system, rho $(\rho)$ is the magnitude velocity and theta $(\theta)$ is the angle between the flow and the Y-axis. Phi $(\phi)$ is the angle of flow through the screens, where $0^{\circ}$ is flow up the screens parallel to the face of the screens, $90^{\circ}$ is perpendicular to the screens, and $180^{\circ}$ is flow down the screens parallel to the face of the screens. 


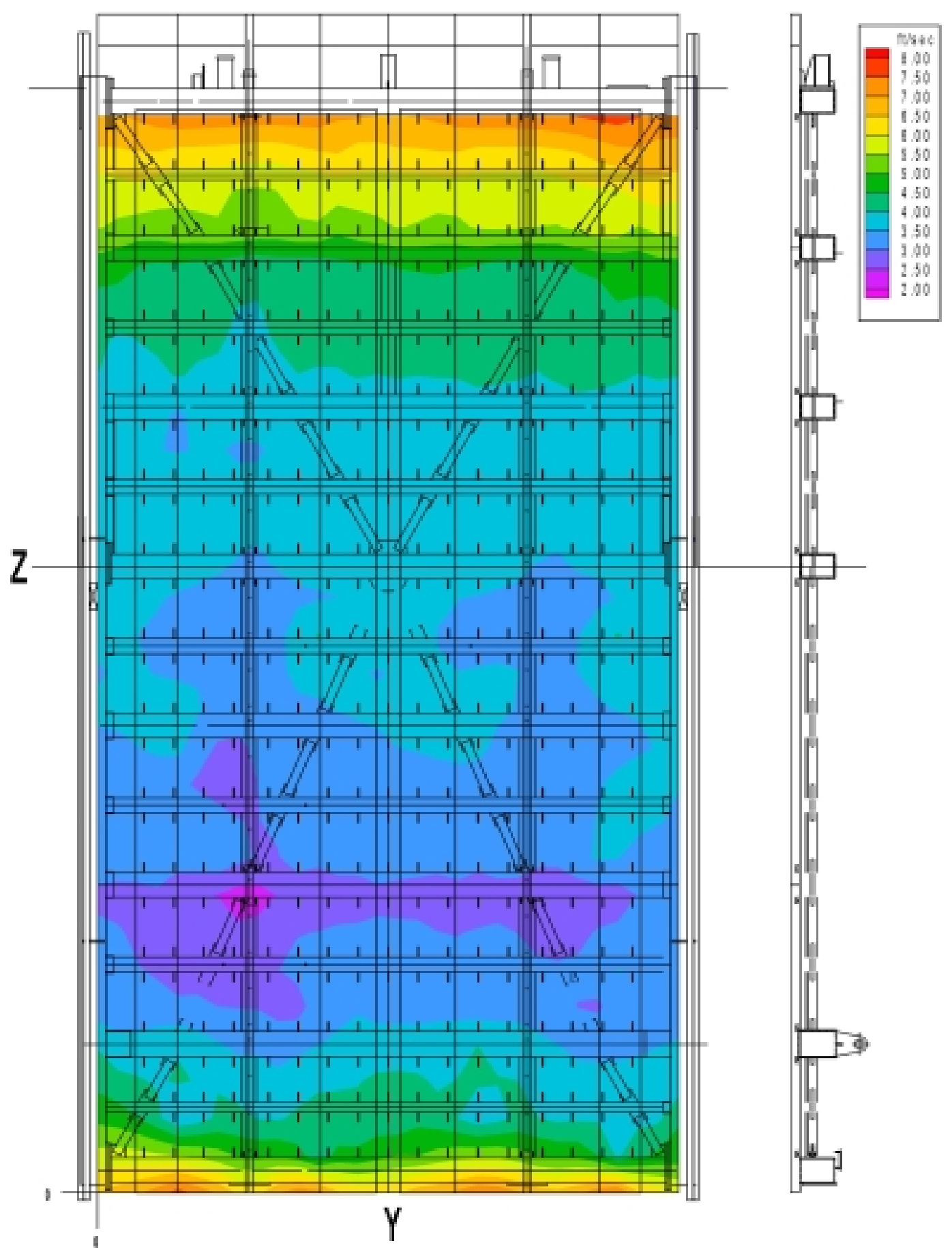

Figure 13. Contour Plot of Magnitude Velocity (ft/sec) 6.75 in. from the Surface of the Bar Screens on the ESBS at 155 MW-Turbine Load 


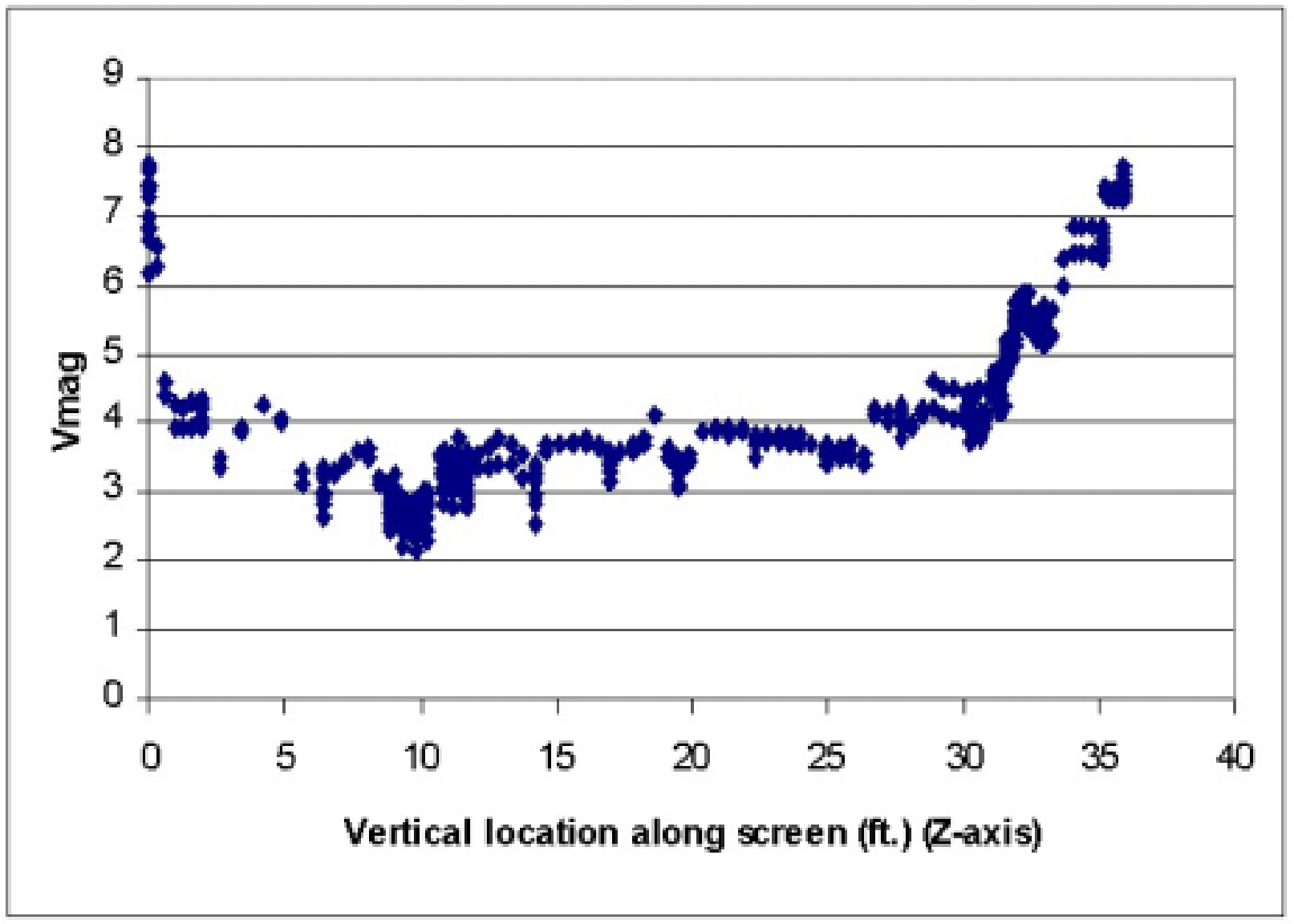

Figure 14. Magnitude Water Velocity (ft/sec) from the Lower End ( $0 \mathrm{ft})$ to $36 \mathrm{ft}$ up the ESBS. Measurements were collected 6.75 in. from the surface of the bar screens at $155 \mathrm{MW}$. 

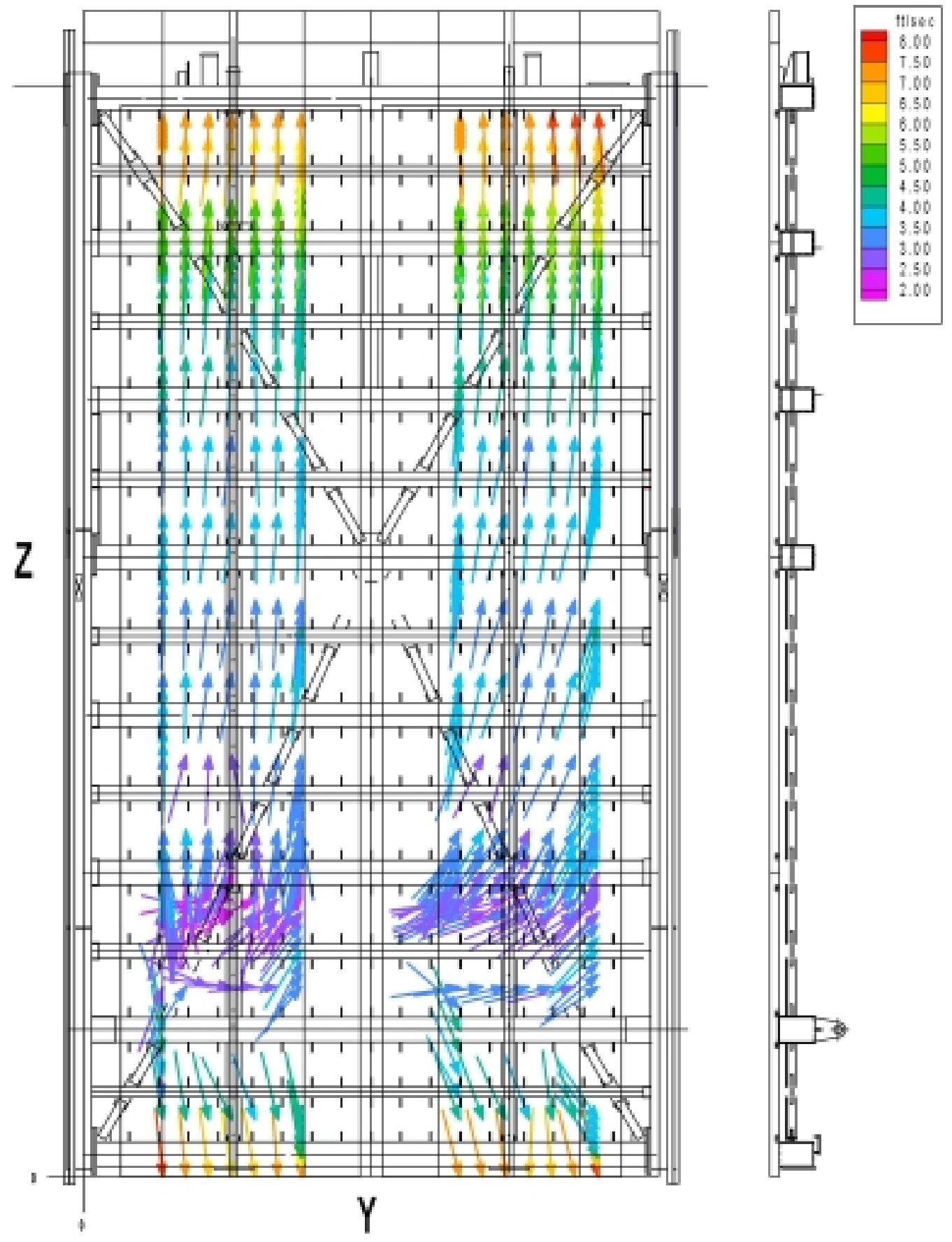

Figure 15. Vector Water Velocity (ft/sec) 6.75 in. from the Surface of the Bar Screens on the ESBS at 155-MW Turbine Load. Vector color is a function of magnitude water velocity $(\mathrm{ft} / \mathrm{sec})$ and vector direction is the direction of flow in the $\mathrm{Y} / \mathrm{Z}$ plane relative to $\mathrm{ADV}$ orientation. 

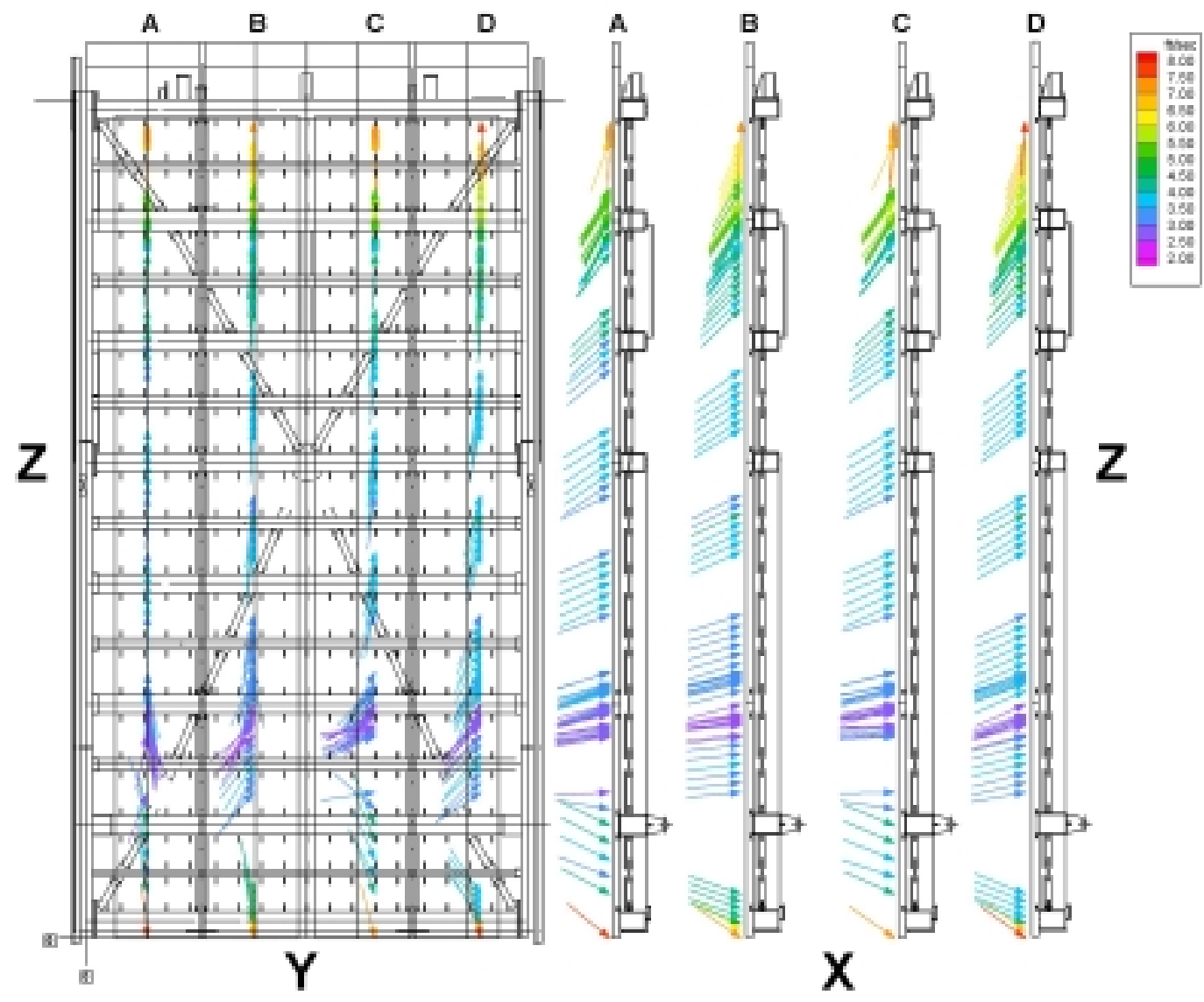

Figure 16. The Face View of the ESBS Shows Vector Water Velocities (ft/sec) in the Y/Z plane, 6.75 in. from the Surface of the Bar Screens, in Four Vertical Zones at $2.67 \mathrm{ft}(\mathrm{A}), 7.33 \mathrm{ft}(\mathrm{B}), 12.67 \mathrm{ft}(\mathrm{C})$, and $17.33 \mathrm{ft}(\mathrm{D})$ across the ESBS. The four side views of the ESBS show vector velocities in the X/Z plane relative to the orientation of the ADV at $155 \mathrm{MW}$ turbine load. Each side view corresponds by letter with a zone of the face view. Vector color is a function of magnitude velocity $(\mathrm{ft} / \mathrm{sec})$ and vector direction represents the direction of bulk flow. 


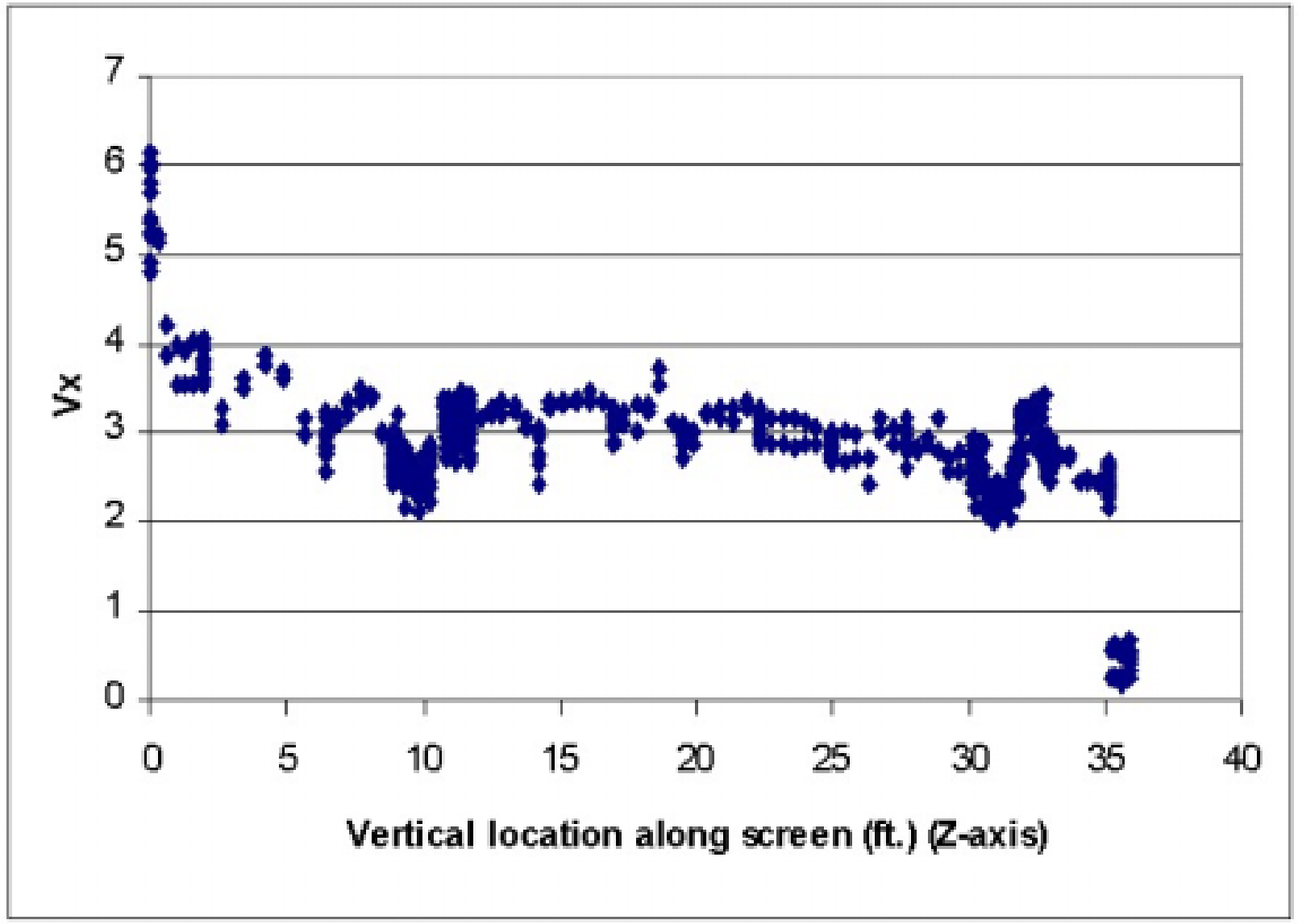

Figure 17. Vector Water Velocity ( $\mathrm{ft} / \mathrm{sec}$ ) in the X-Axis (perpendicular to the bar screens) from the Lower End $(0 \mathrm{ft})$ to $36 \mathrm{ft}$ up the ESBS. Measurements were collected $6.75 \mathrm{in}$. from the surface of the bar screens at 155-MW turbine load. 


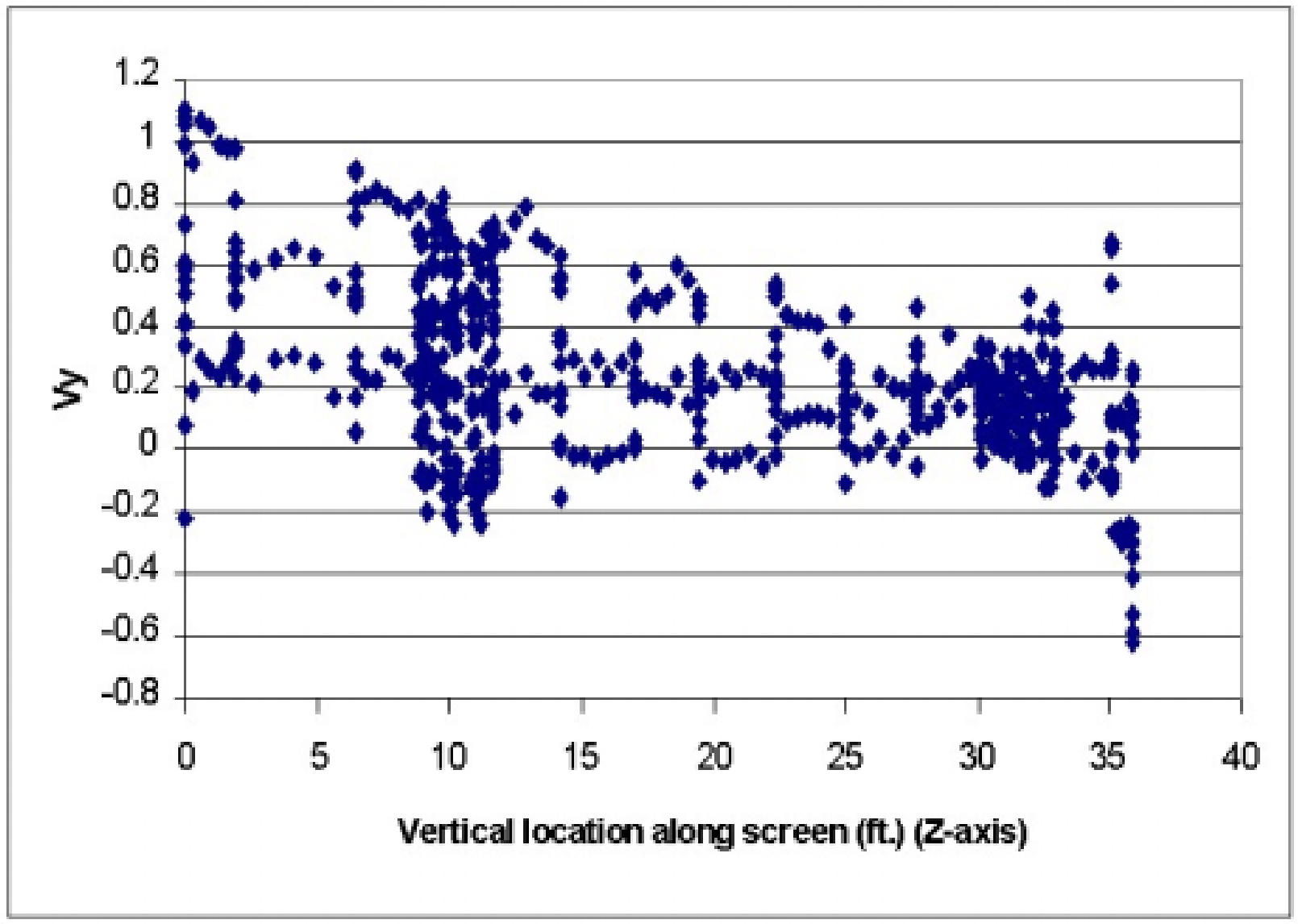

Figure 18. Vector Water Velocity (ft/sec) in the Y-Axis (horizontal to the bar screens) from the Lower end $(0 \mathrm{ft})$ to $36 \mathrm{ft}$ up the ESBS. Measurements were collected 6.75 in. from the surface of the bar screens at 155-MW turbine load. 


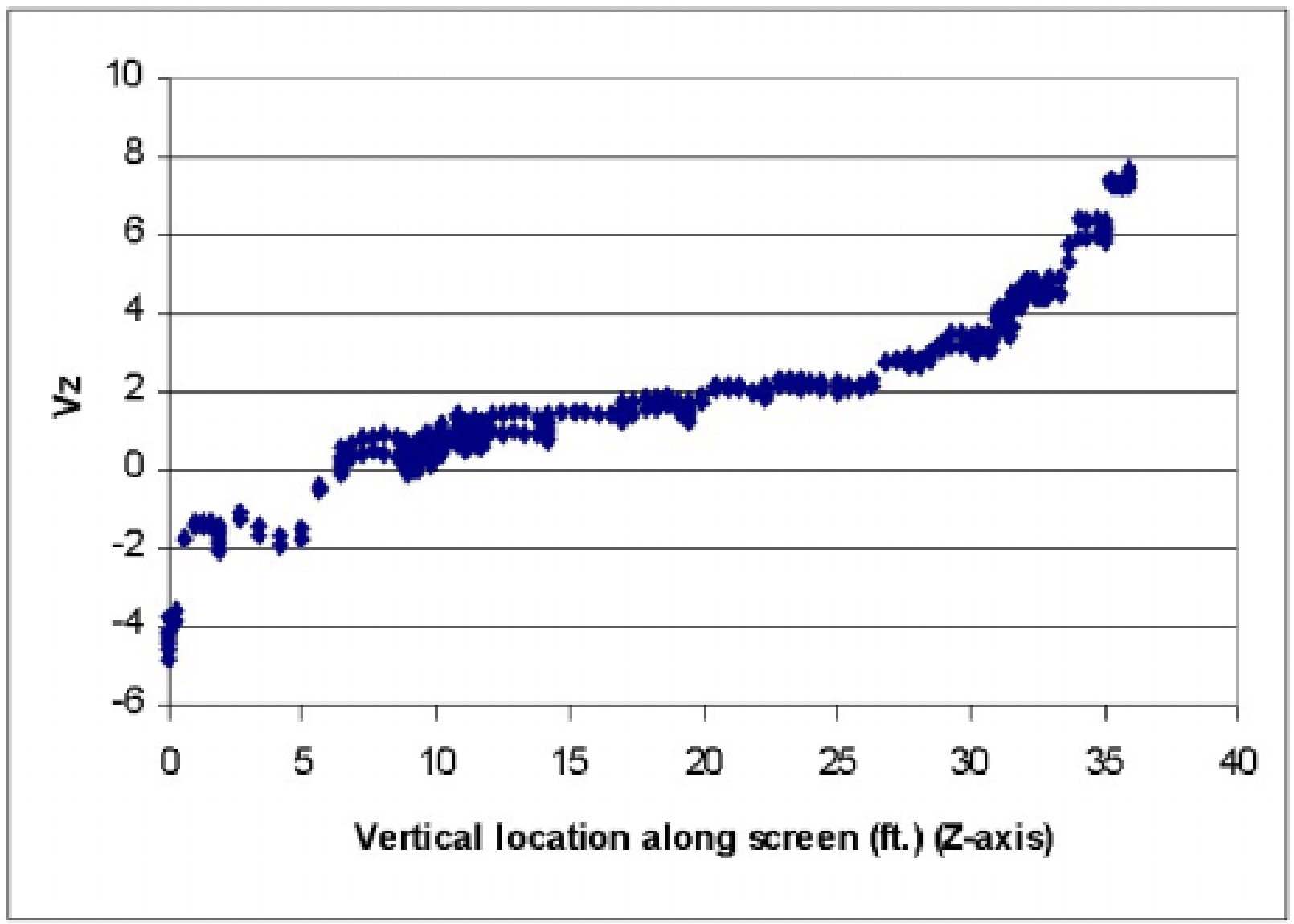

Figure 19. Vector Water Velocity ( $\mathrm{ft} / \mathrm{sec}$ ) in the Z-Axis (longitudinal to the bar screens) from the Lower End ( $0 \mathrm{ft})$ to $36 \mathrm{ft}$ up the ESBS. Measurements were collected $6.75 \mathrm{in}$. from the surface of the bar screens at 155-MW turbine load. 


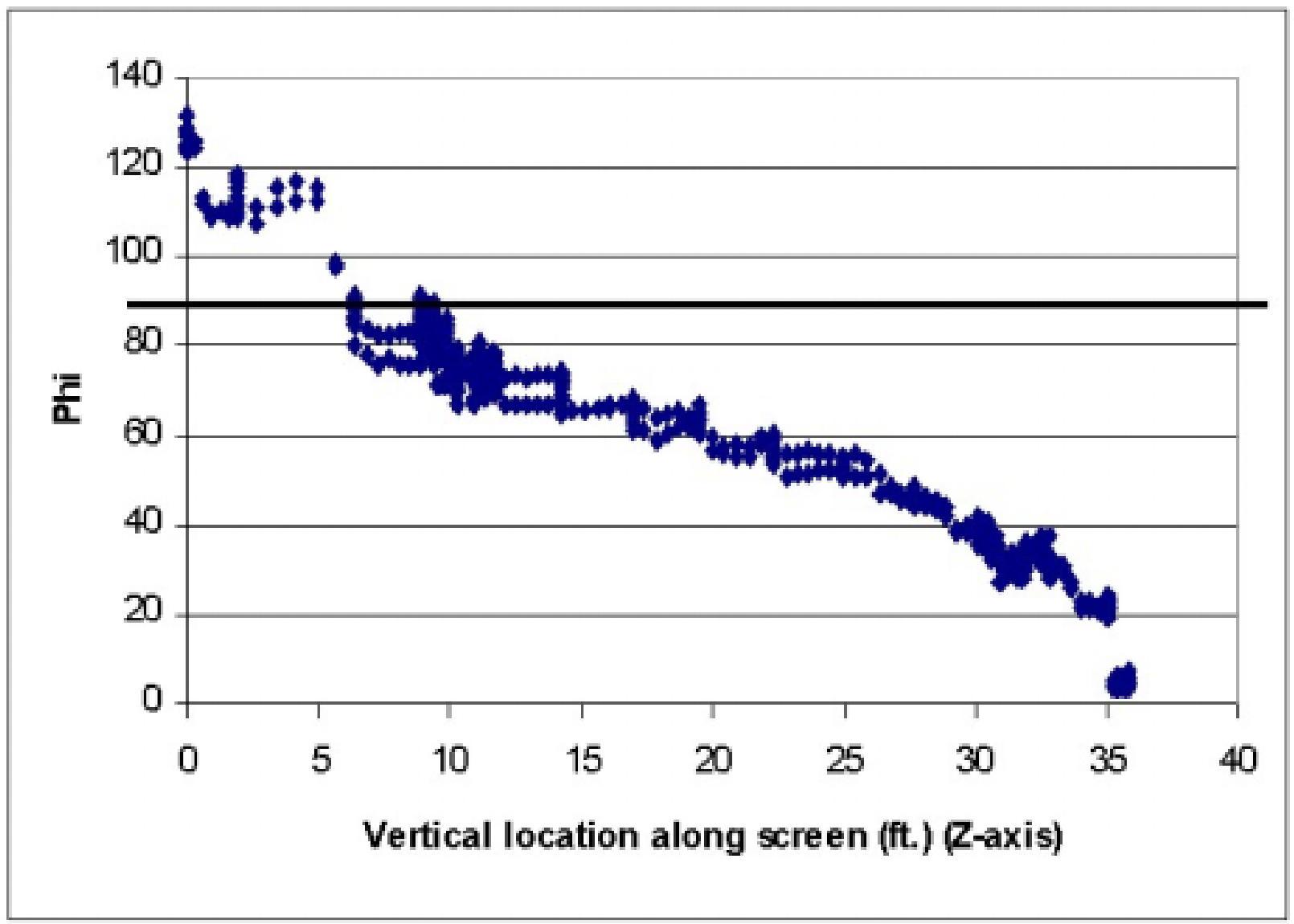

Figure 20. Angular Direction of Flow Phi $(\phi)$ in the Perpendicular Plane (X-axis) to the Bar Screens at 155-MW Turbine Load. The line at $90^{\circ}$ indicates the division between flow up the screens toward the gate well $\left(<90^{\circ}\right)$ and down the screen $\left(>90^{\circ}\right)$. If the angular direction of flow is $90^{\circ}$ then flow is perpendicular to the bar screens. 


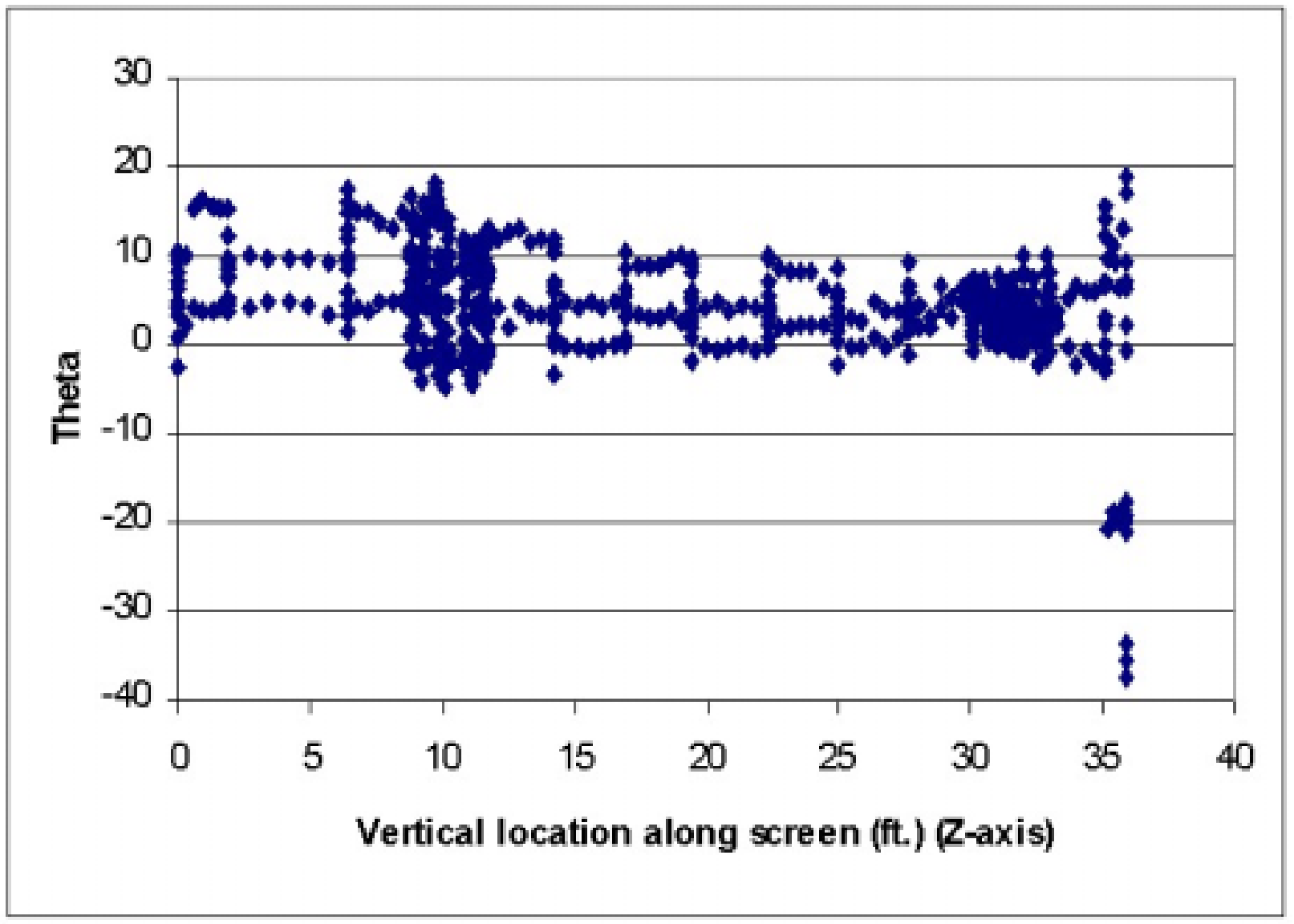

Figure 21. Angular Direction of Flow Theta $(\theta)$ in the Horizontal Plane (Y-axis) to the Bar Screens at $155-\mathrm{MW}$ turbine load. When Theta is greater than $0^{\circ}$ the flow is moving toward the right side of the ESBS across the bar screen and to the left when the Theta is less than $0^{\circ}$. When Theta equals $0^{\circ}$ flow is up the bar screens with no horizontal movement. 

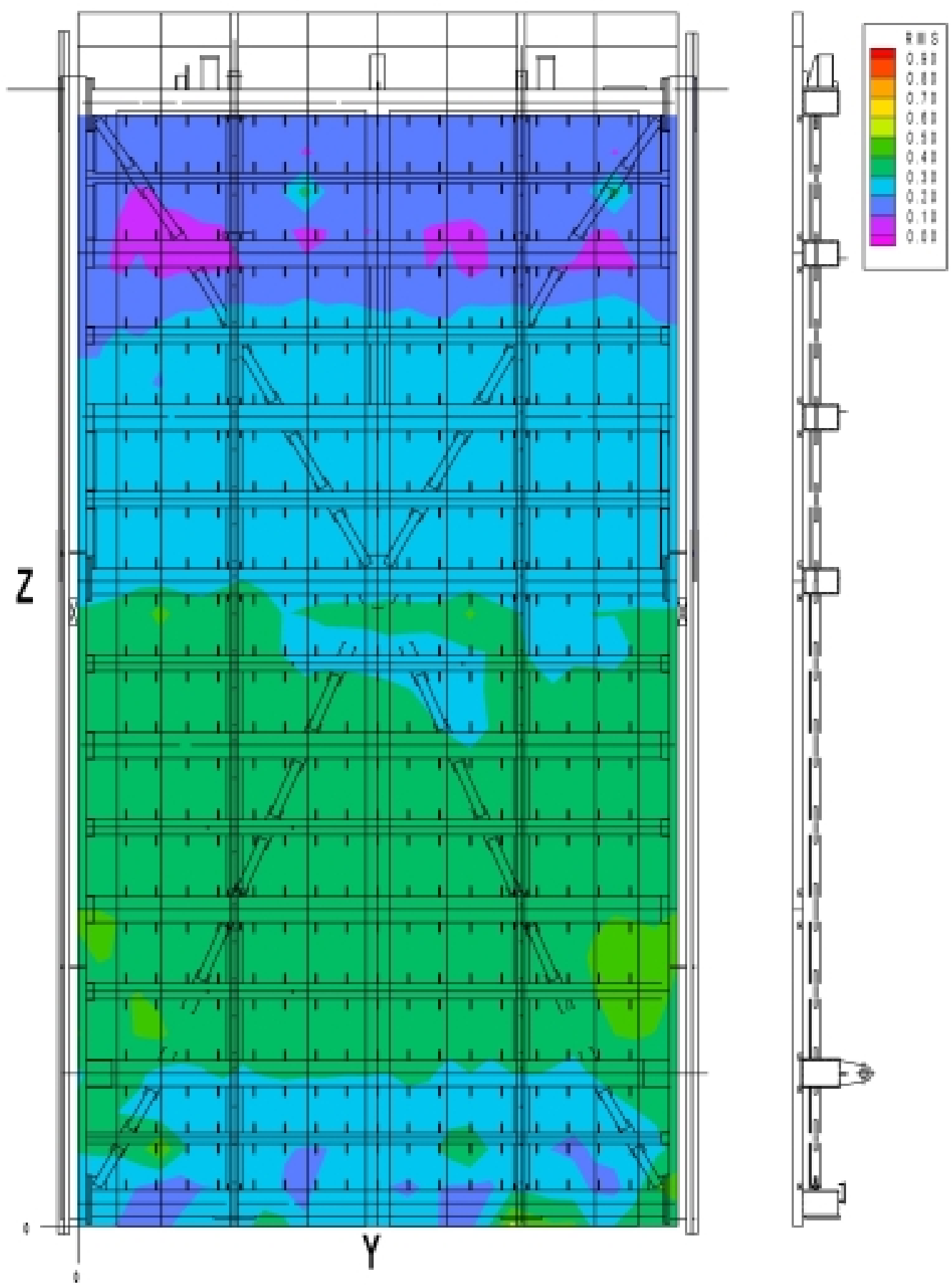

Figure 22. Contour Plot of Magnitude Flow RMS Turbulence 6.75 in. from the Surface of the Bar Screens on the ESBS at 155-MW Turbine Load 

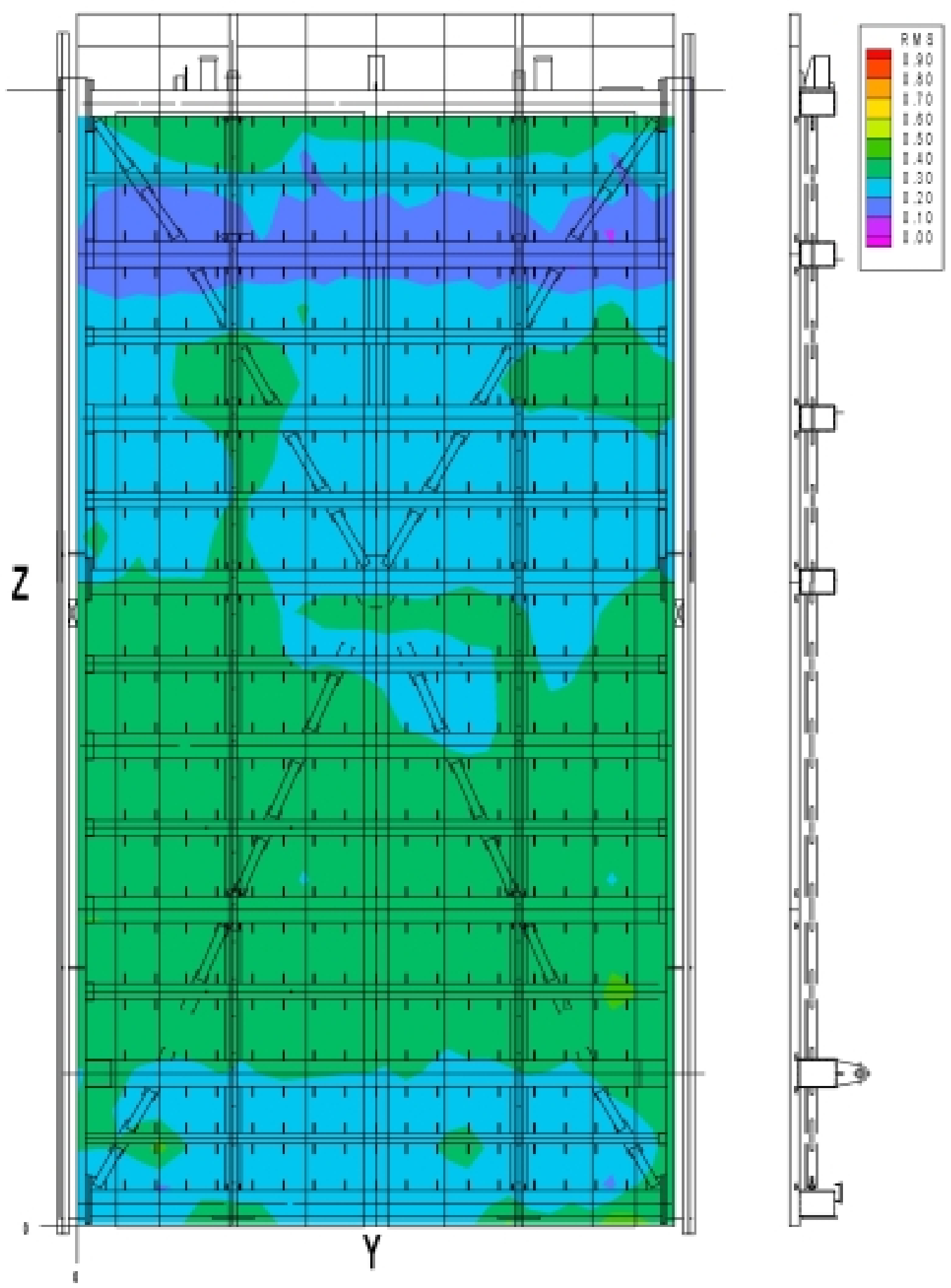

Figure 23. Contour Plot of RMS Turbulence into the Screen (x-axis) 6.75 in. from the Surface of the Bar Screens on the ESBS at 155-MW Turbine Load 

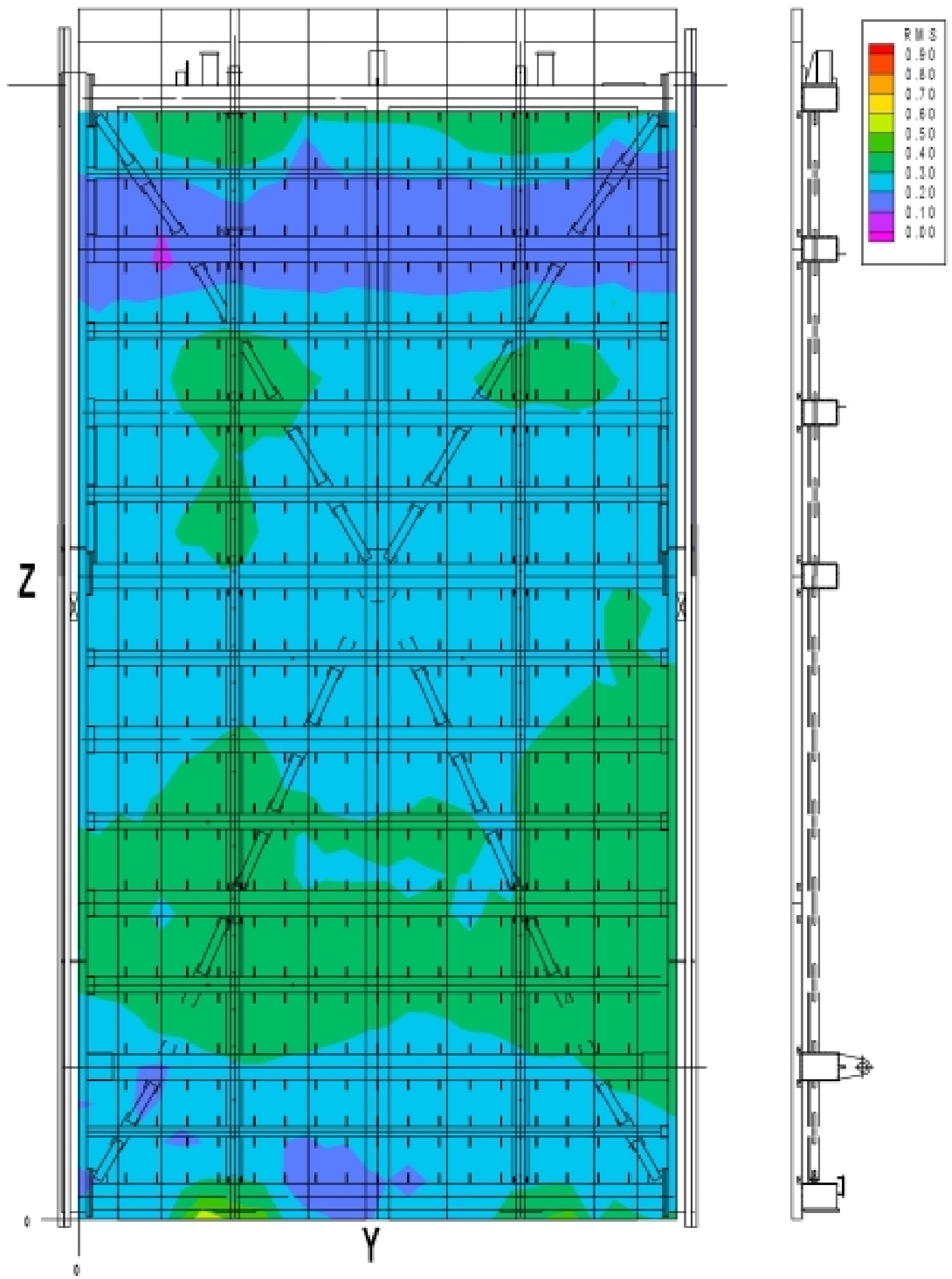

Figure 24. Contour Plot of RMS Turbulence across the Screen (y-axis) 6.75 in. from the Surface of the Bar Screens on the ESBS at 155-MW Turbine Load 

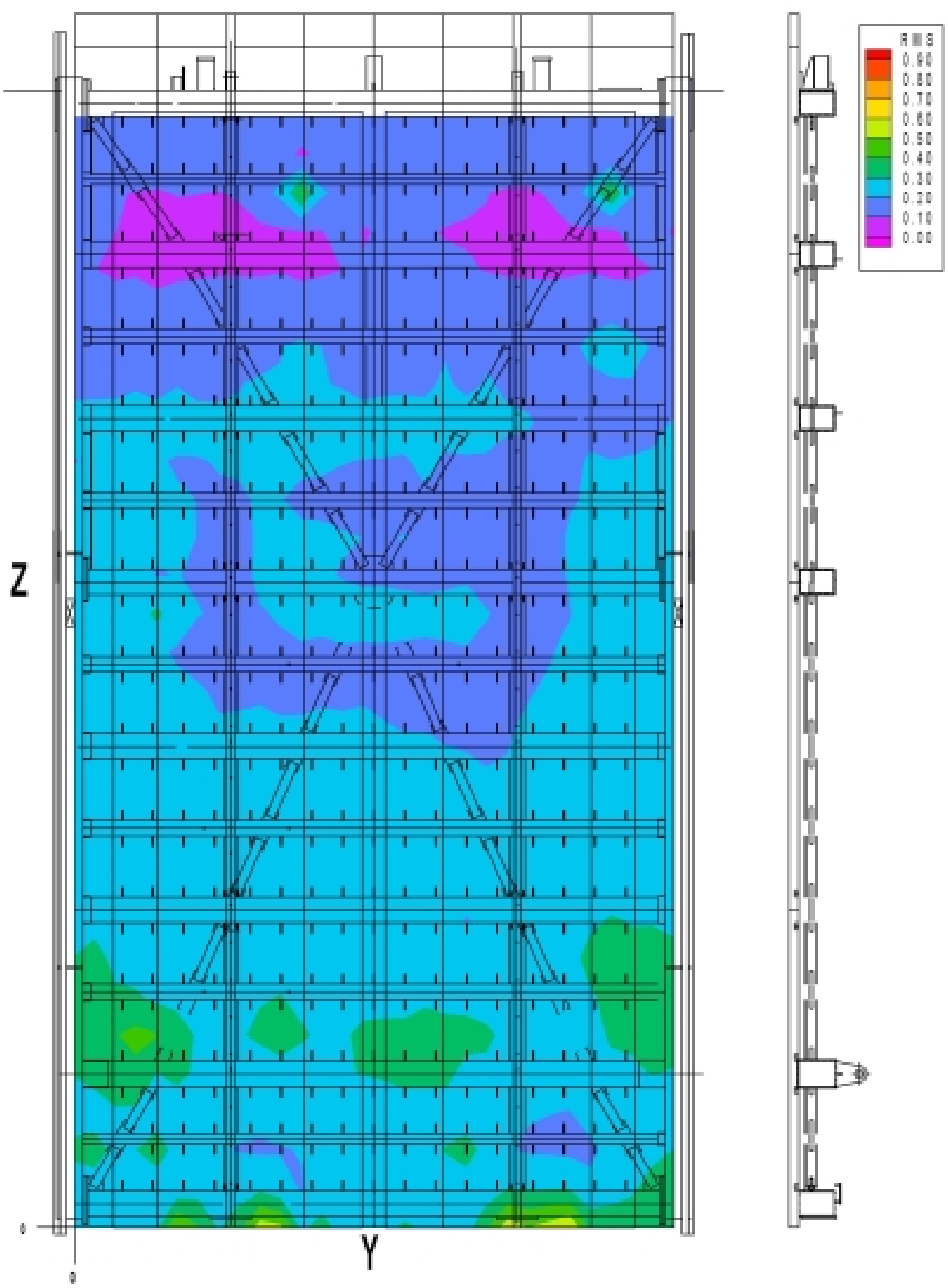

Figure 25. Contour Plot of RMS Turbulence Longitudinal to the Screen (z-axis) 6.75 in. from the Surface of the Bar Screens on the ESBS at 155-MW Turbine Load 

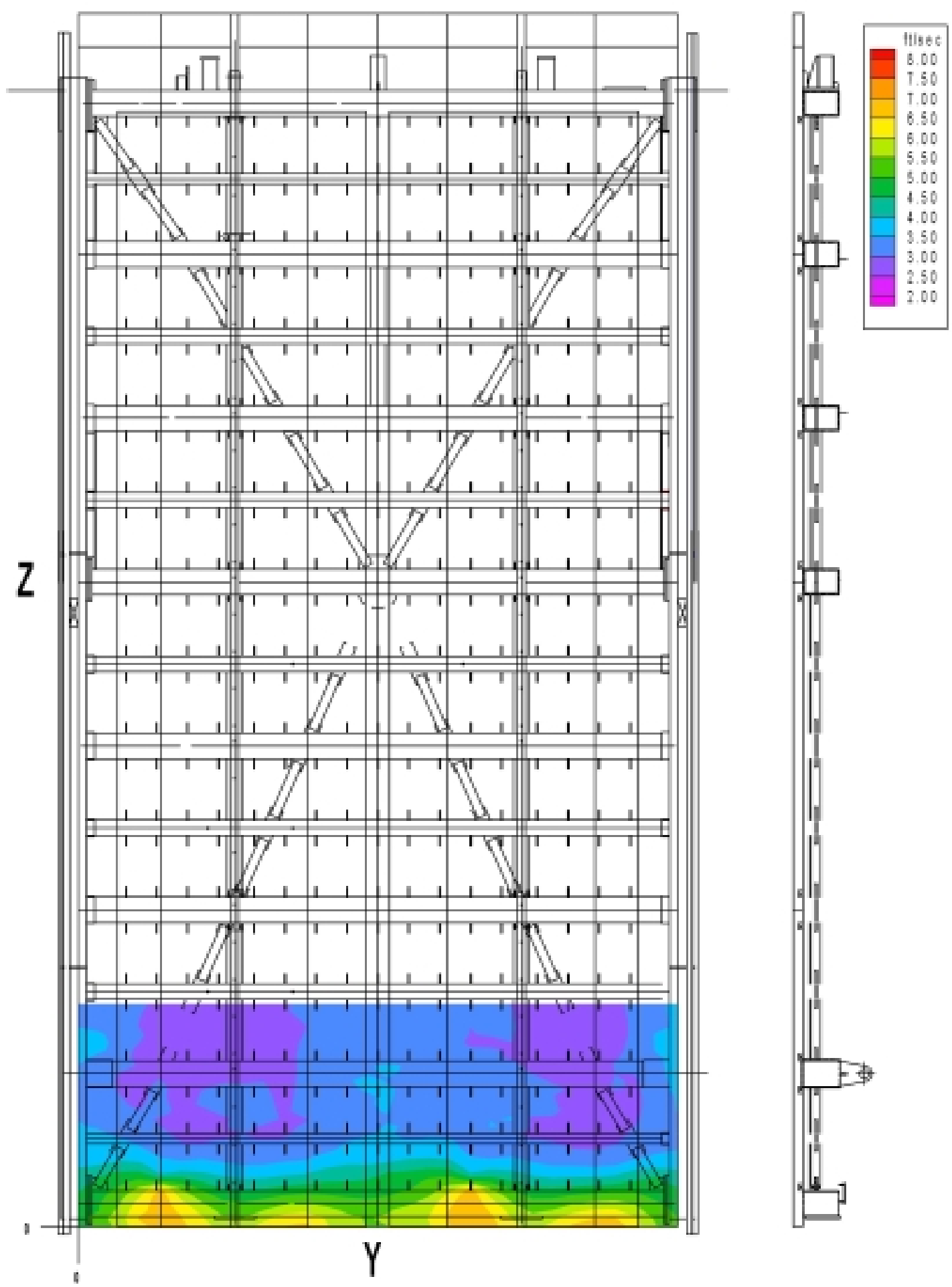

Figure 26. Contour Plot of Magnitude Velocity (ft/sec) 6.75 in. from the Surface of the Bar Screens on the ESBS at 138-MW Turbine Load 

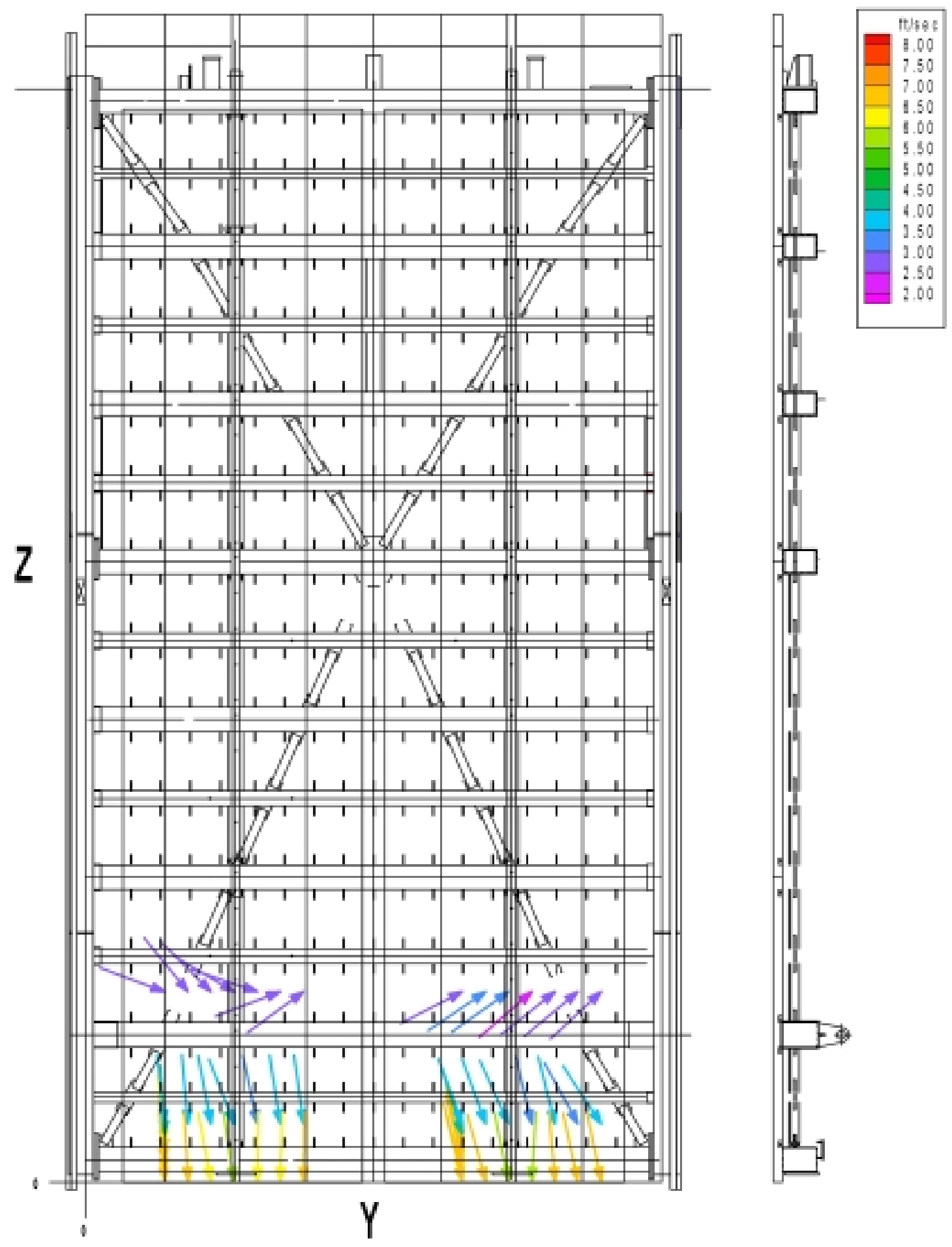

Figure 27. Vector Water Velocity (ft/sec) 6.75 in. from the Surface of the Bar Screens on the ESBS at 138-MW Turbine Load. Vector color is a function of magnitude water velocity $(\mathrm{ft} / \mathrm{sec}$ ) and vector direction is the direction of flow in the $\mathrm{Y} / \mathrm{Z}$ plane relative to ADV orientation. 

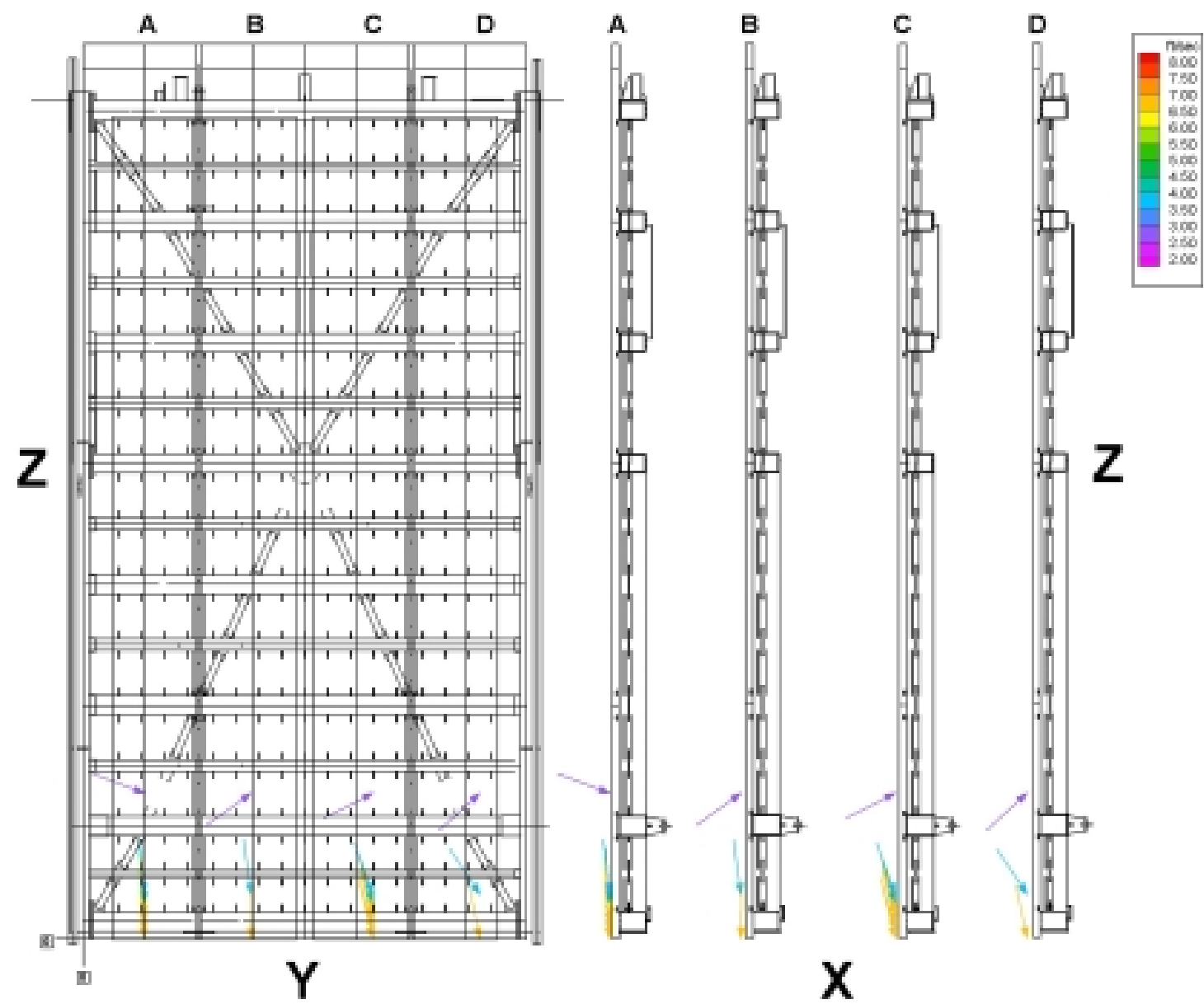

Figure 28. The Face View of the ESBS Shows Vector Water Velocities (ft/sec) in the Y/Z Plane, 6.75 in. from the Surface of the Bar Screens, in Four Vertical Zones at $2.67 \mathrm{ft}(\mathrm{A}), 7.33 \mathrm{ft}(\mathrm{B}), 12.67 \mathrm{ft}(\mathrm{C})$, and $17.33 \mathrm{ft}(\mathrm{D})$ across the ESBS. The four side views of the ESBS show vector velocities in the X/Z plane relative to the orientation of the ADV at $138 \mathrm{MW}$ turbine load. Each side view corresponds by letter with a zone of the face view. Vector color is a function of magnitude velocity $(\mathrm{ft} / \mathrm{sec})$ and vector direction represents the direction of bulk flow. 

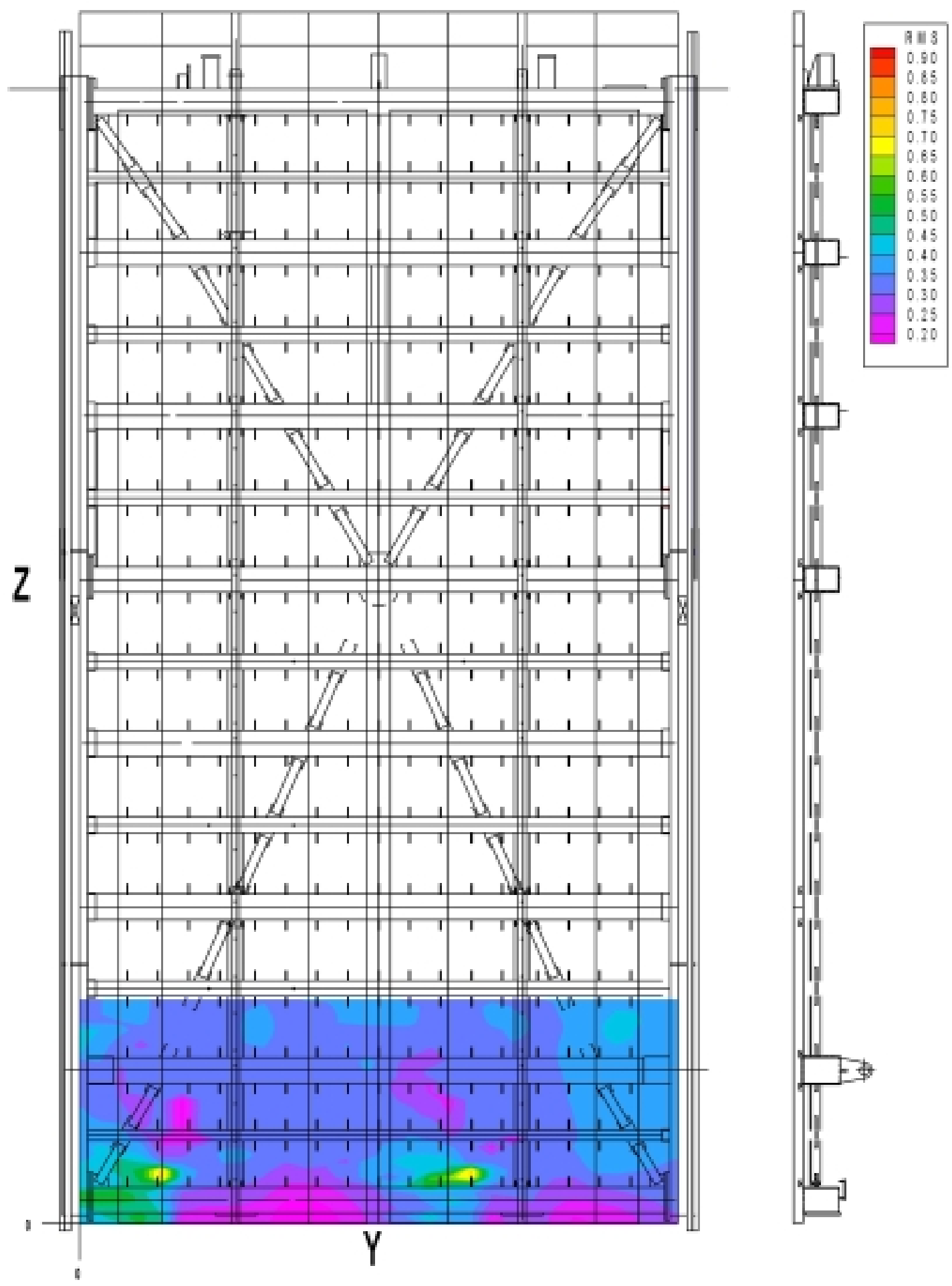

Figure 29. Contour Plot of Magnitude Flow RMS Turbulence 6.75 in. from the Surface of the Bar Screens on the ESBS at 138-MW Turbine Load 

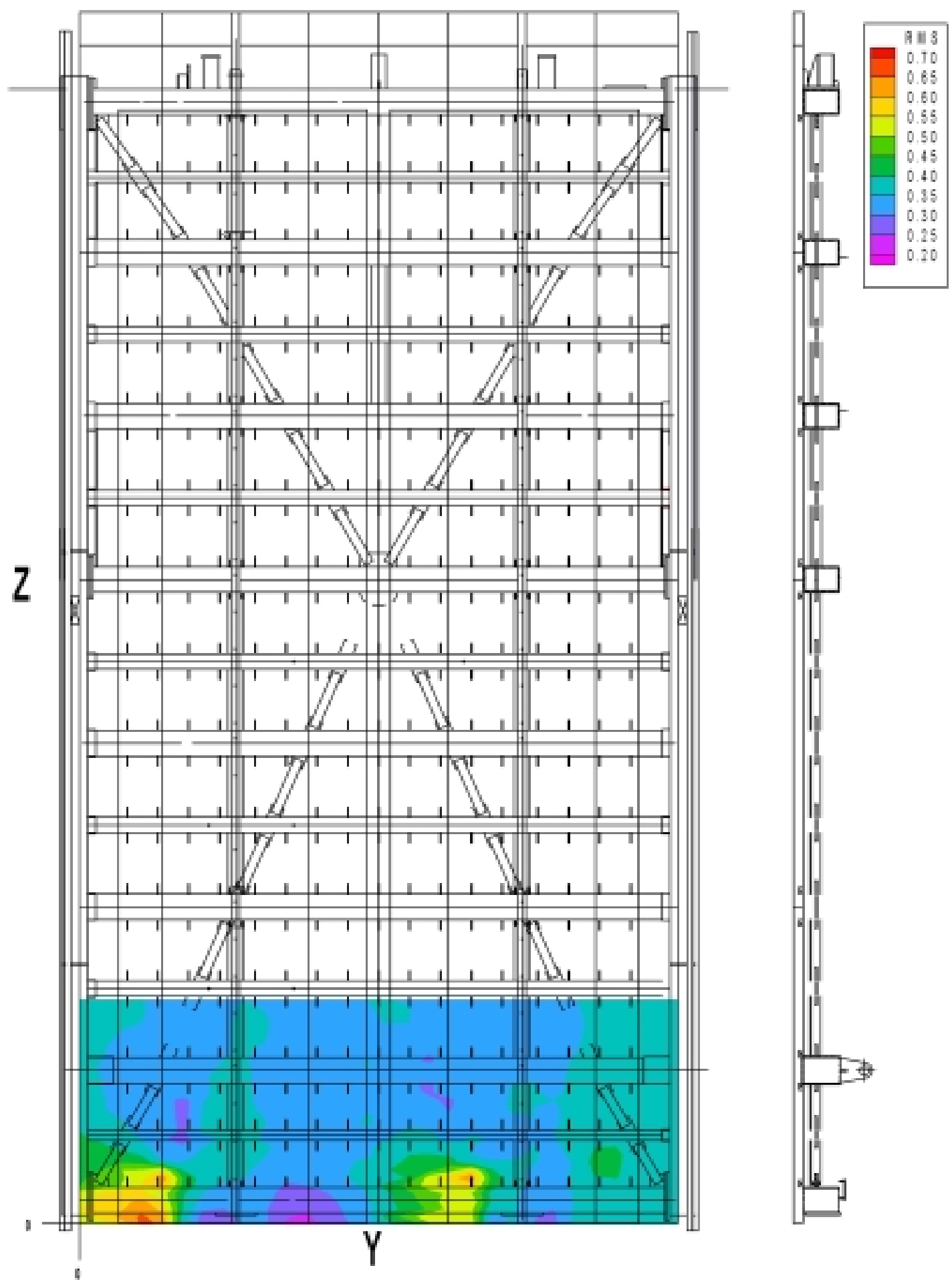

Figure 30. Contour Plot of RMS Turbulence into the Screen (x-axis) 6.75 in. from the Surface of the Bar Screens on the ESBS at 138-MW Turbine Load 

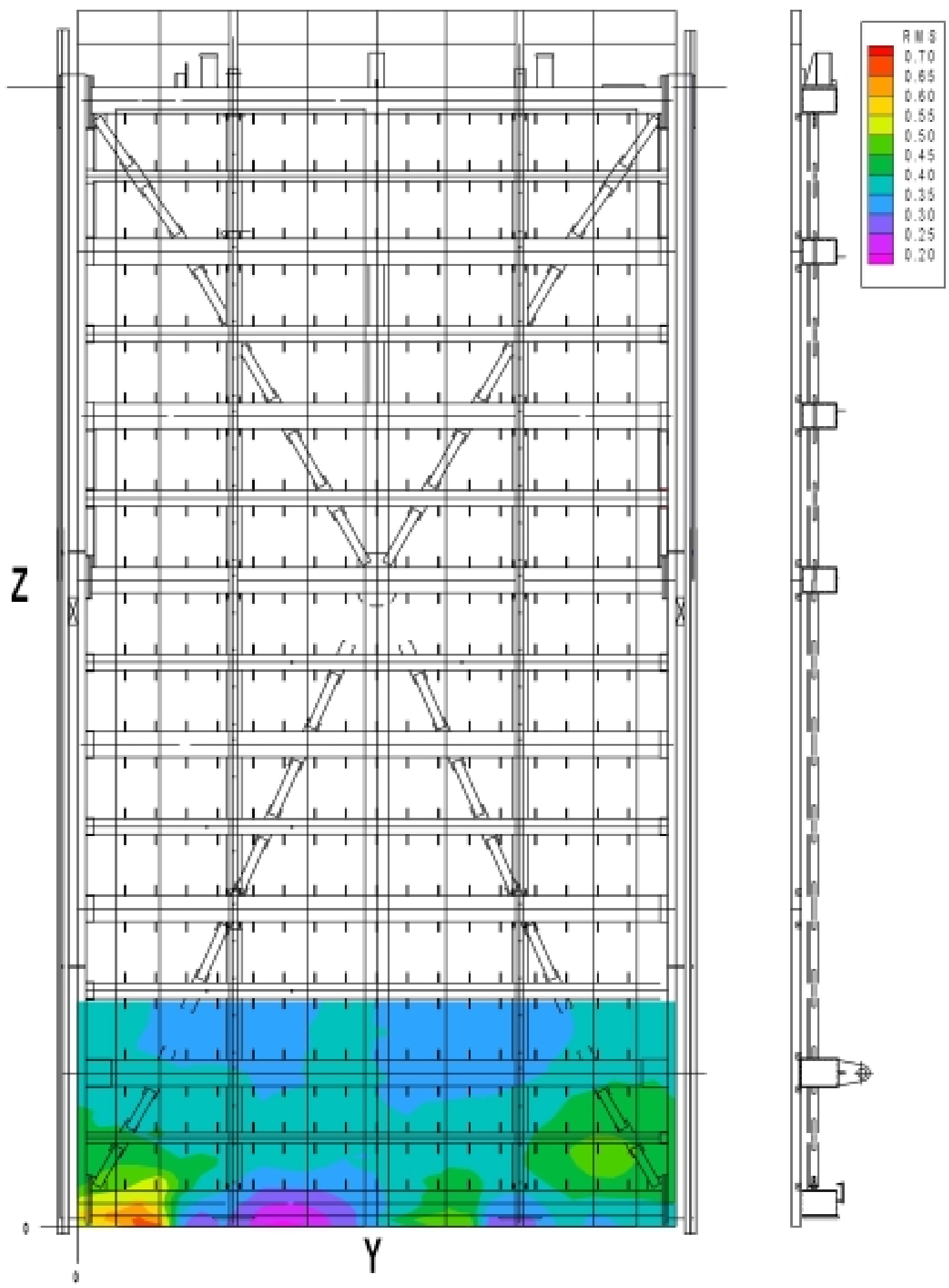

Figure 31. Contour Plot of RMS Turbulence across the Screen (y-axis) 6.75 in. from the Surface of the Bar Screens on the ESBS at 138-MW Turbine Load 

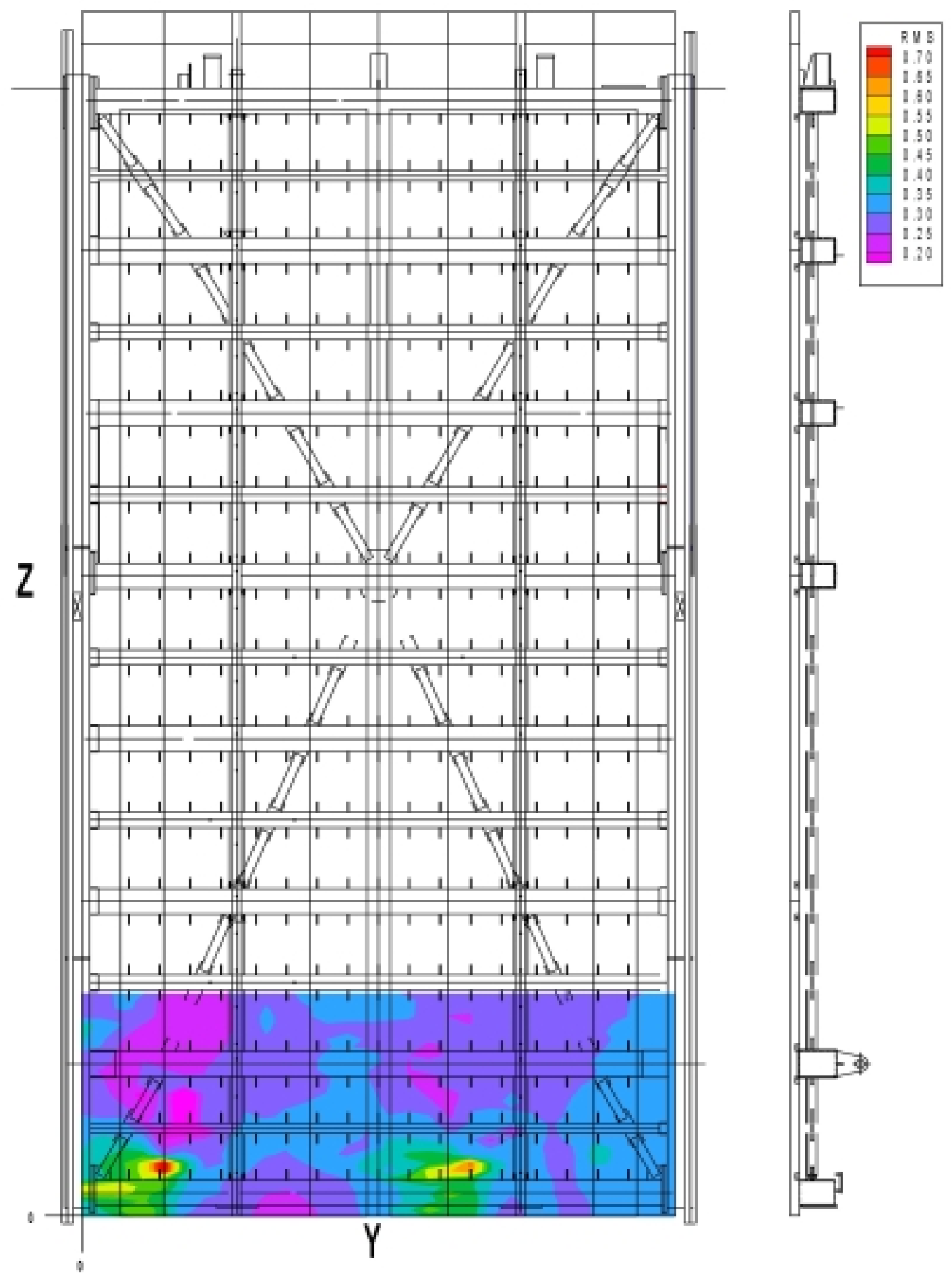

Figure 32. Contour Plot of RMS Turbulence Longitudinal to the Screen (z-axis) 6.75 in. from the Surface of the Bar Screens on the ESBS at 138-MW Turbine Load 


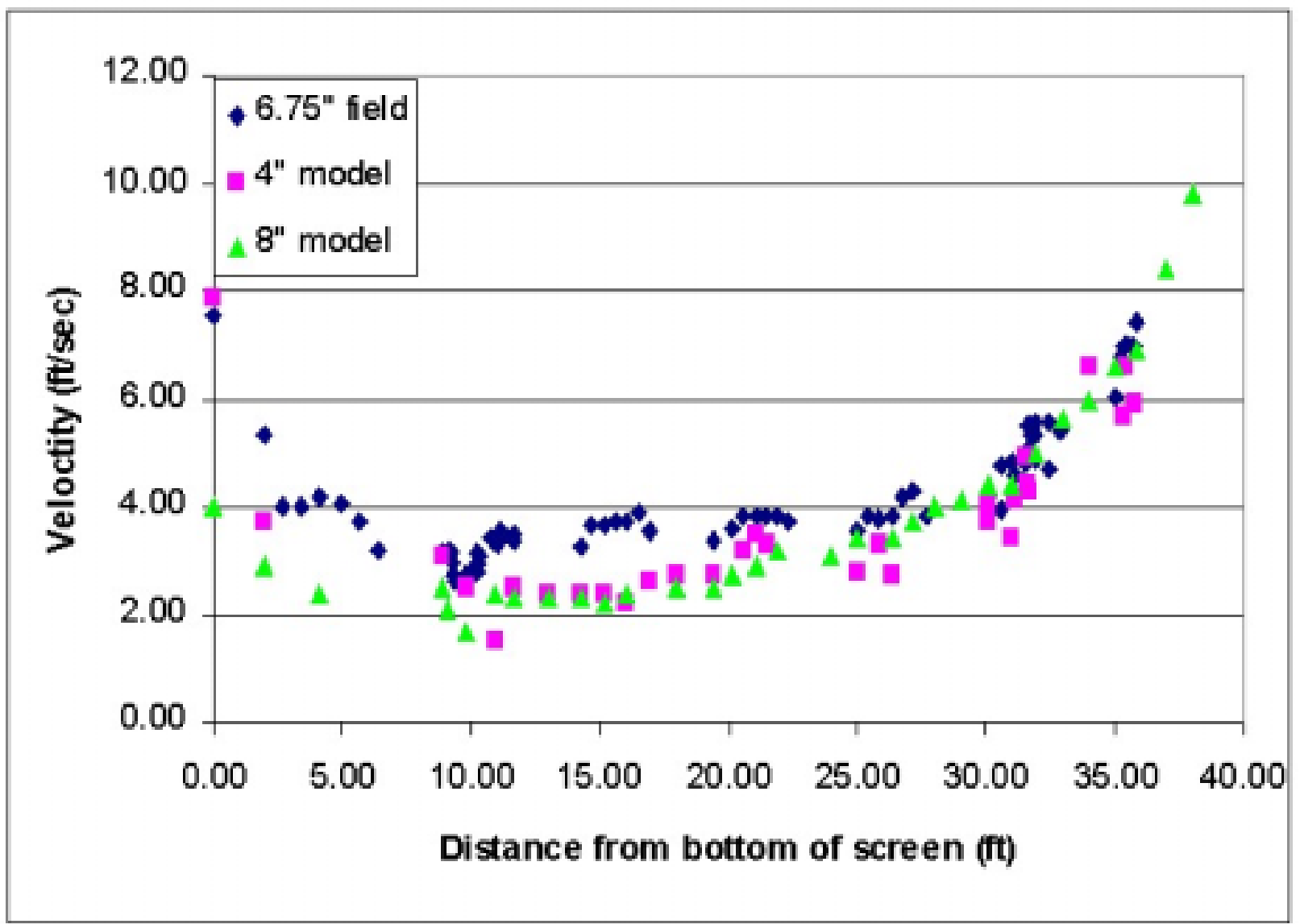

Figure 33. Comparison of Velocity Measurements between Data Collected in the Field 6.75 in. from the Bar Screens at 21,000 cfs and Physical Model Data Collected at scale 4 in. and 8 in. from the Screen at 20,800 cfs Longitudinally (z-axis) on the ESBS. 


\section{Appendix A}

\section{X, Y, and Z Vector Velocities at 155 MW Turbine Load}




\section{Appendix A}

\section{$\mathrm{X}, \mathrm{Y}$, and Z Vector Velocities at $155 \mathrm{MW}$ Turbine Load}

The table in this appendix lists $\mathrm{X}, \mathrm{Y}$, and $\mathrm{Z}$ vector velocities ( $\mathrm{ft} / \mathrm{sec}$ ) for flow data collected at locations along the bar screen surface of the ESBS at $155 \mathrm{MW}$ turbine load. Mean, minimum, and maximum vector velocities along with the standard error are provided for each sample location.

\begin{tabular}{|c|c|c|c|c|c|c|c|c|c|c|c|c|c|}
\hline \multicolumn{2}{|c|}{ Location (ft.) } & \multicolumn{4}{|c|}{ X Vector (ft./sec) } & \multicolumn{4}{|c|}{ Y Vector (ft./sec) } & \multicolumn{4}{|c|}{ Z Vector (ft./sec) } \\
\hline Vertical & Horizontal & Mean & Min & Max & Stderr & Mean & Min & Max & Stderr & Mean & Min & Max & Stderr \\
\hline-0.02 & 2.67 & 6.00 & 5.13 & 6.54 & 0.02012 & 0.51 & -0.36 & 1.35 & 0.02135 & -4.87 & -5.58 & -4.29 & 0.02383 \\
\hline-0.02 & 3.45 & 5.71 & 4.94 & 6.44 & 0.02654 & 0.41 & -0.41 & 1.54 & 0.03703 & -4.45 & -5.67 & -3.66 & 0.04763 \\
\hline-0.02 & 4.22 & 5.35 & 4.30 & 6.14 & 0.03623 & 0.55 & -0.45 & 2.05 & 0.05466 & -4.18 & -5.13 & -3.40 & 0.03047 \\
\hline-0.02 & 5.00 & 4.82 & 4.09 & 5.77 & 0.02948 & 0.59 & -0.41 & 1.83 & 0.03644 & -3.76 & -4.43 & -2.93 & 0.02180 \\
\hline-0.02 & 5.78 & 5.22 & 4.18 & 6.34 & 0.03398 & 0.73 & -0.18 & 1.78 & 0.04329 & -4.25 & -5.21 & -2.91 & 0.04688 \\
\hline-0.02 & 6.55 & 5.80 & 5.08 & 6.55 & 0.02383 & 0.34 & -0.34 & 1.32 & 0.02638 & -4.58 & -5.62 & -3.59 & 0.04654 \\
\hline-0.02 & 7.33 & 5.95 & 5.32 & 6.79 & 0.01573 & 0.08 & -0.54 & 0.61 & 0.01360 & -4.15 & -4.82 & -3.45 & 0.01410 \\
\hline-0.02 & 12.67 & 6.02 & 5.12 & 6.83 & 0.02331 & 1.10 & 0.39 & 1.57 & 0.01969 & -4.27 & -4.85 & -3.59 & 0.02240 \\
\hline-0.02 & 13.45 & 5.99 & 4.89 & 7.12 & 0.03365 & 0.98 & 0.12 & 1.97 & 0.02602 & -4.12 & -5.17 & -3.09 & 0.03615 \\
\hline-0.02 & 14.22 & 5.41 & 4.49 & 6.66 & 0.03781 & 0.61 & -0.63 & 1.67 & 0.03727 & -4.37 & -5.60 & -3.28 & 0.05273 \\
\hline-0.02 & 15.00 & 4.93 & 4.26 & 5.60 & 0.02334 & -0.22 & -1.10 & 0.61 & 0.02945 & -4.43 & -5.52 & -3.66 & 0.03691 \\
\hline-0.02 & 15.78 & 5.25 & 4.48 & 6.22 & 0.03559 & 0.42 & -0.89 & 1.35 & 0.04424 & -4.19 & -5.41 & -2.93 & 5900 \\
\hline-0.02 & 16.55 & 5.98 & 5.25 & 6.90 & 0.02582 & 1.05 & 0.29 & 1.74 & 0.02414 & -4.19 & -5.16 & -3.49 & 0.03134 \\
\hline-0.02 & 17.33 & 6.16 & 5.28 & 7.92 & 0.02383 & 1.08 & -0.01 & 2.58 & 0.02200 & -4.39 & -5.34 & -3.54 & 0.02222 \\
\hline 0.30 & 7.33 & 5.15 & 4.39 & 5.84 & 0.09680 & 0.19 & -0.16 & 0.51 & 0.04605 & -3.58 & -4.57 & -2.19 & 0.17154 \\
\hline 0.30 & 17.33 & 5.21 & 3.88 & 6.45 & 0.17892 & 0.93 & 0.44 & 1.49 & 0.06861 & -3.82 & -4.67 & -2.36 & 0.14849 \\
\hline 0.63 & 7.33 & 4.22 & 3.82 & 4.66 & 0.06580 & 0.30 & 0.05 & 0.67 & 0.04174 & -1.72 & -2.27 & -1.16 & 0.09042 \\
\hline 0.63 & 17.33 & 3.88 & 2.94 & 4.48 & 0.10278 & 1.07 & 0.68 & 1.47 & 0.05734 & \begin{tabular}{|l|}
-1.77 \\
\end{tabular} & -2.65 & -1.03 & 1173 \\
\hline 0.95 & 7.33 & 4.00 & 3.74 & 4.45 & 0.03598 & 0.27 & 0.04 & 0.55 & 0.03244 & \begin{tabular}{|l|}
-1.38 \\
\end{tabular} & -1.77 & $\begin{array}{l}-1.09 \\
\end{array}$ & 0.04350 \\
\hline 0.95 & 17.33 & 3.54 & 3.14 & 3.88 & 0.05132 & 1.05 & 0.64 & 1.58 & 0.05761 & \begin{tabular}{|l|}
-1.31 \\
\end{tabular} & -1.54 & -1.02 & 0.04036 \\
\hline 1.27 & 7.33 & 3.92 & 3.53 & 4.26 & 0.05324 & 0.24 & 0.01 & 0.54 & 0.03977 & -1.45 & -1.73 & -1.07 & 0.04745 \\
\hline 1.27 & 17.33 & 3.54 & 3.25 & 3.90 & 0.04034 & 0.98 & 0.44 & 1.47 & 0.06316 & \begin{tabular}{|l|}
-1.35 \\
\end{tabular} & -1.84 & -1.07 & 0.05737 \\
\hline 1.60 & 7.33 & 4.03 & 3.74 & 4.31 & 0.04054 & 0.29 & -0.05 & 0.55 & 0.04433 & \begin{tabular}{|l|}
-1.42 \\
\end{tabular} & -1.75 & -1.10 & 0.03361 \\
\hline 1.60 & 17.33 & 3.54 & 3.25 & 4.06 & 0.05275 & 0.97 & 0.48 & 1.39 & 0.05408 & \begin{tabular}{|l|}
-1.37 \\
\end{tabular} & -1.98 & -1.00 & 0.05247 \\
\hline 1.92 & 2.67 & 3.77 & 3.22 & 4.12 & 0.00963 & 0.34 & -0.23 & 0.96 & 0.01198 & -1.98 & -2.41 & -1.48 & 0.00971 \\
\hline 1.92 & 3.45 & 3.78 & 3.19 & 4.21 & 0.02863 & 0.32 & -0.10 & 0.71 & 0.02441 & -2.05 & -2.30 & -1.67 & 0.02143 \\
\hline 1.92 & 4.22 & 3.74 & 3.28 & 4.22 & 0.03013 & 0.48 & -0.08 & 0.93 & 0.02883 & -2.03 & -2.39 & -1.72 & 0.02638 \\
\hline 1.92 & 5.00 & 3.75 & 3.27 & 4.17 & 0.03035 & 0.81 & 0.17 & 1.16 & 0.02774 & \begin{tabular}{|l|}
-1.67 \\
\end{tabular} & -2.33 & -1.31 & 0.02694 \\
\hline 1.92 & 5.78 & 3.61 & 3.28 & 4.18 & 0.02689 & 0.50 & -0.09 & 1.05 & 0.03951 & \begin{tabular}{|l|}
-1.48 \\
\end{tabular} & -1.83 & -1.15 & 0.02351 \\
\hline 1.92 & 6.55 & 3.94 & 3.54 & 4.48 & 0.02929 & 0.35 & 0.03 & 0.67 & 0.02148 & \begin{tabular}{|l|}
-1.39 \\
\end{tabular} & -1.79 & -1.04 & 0.02409 \\
\hline 1.92 & 7.33 & 4.06 & 3.58 & 4.56 & 0.00938 & 0.33 & -0.15 & 0.86 & 0.00941 & -1.41 & -1.89 & -0.90 & 0.00932 \\
\hline 1.92 & 12.67 & 3.85 & 3.31 & 4.38 & 0.01147 & 0.64 & 0.02 & 1.09 & 0.01140 & \begin{tabular}{|l|}
-1.70 \\
\end{tabular} & -2.29 & -1.19 & 0.01180 \\
\hline 1.92 & 13.45 & 3.98 & 3.57 & 4.48 & 0.02695 & 0.59 & 0.19 & 1.14 & 0.02886 & -1.62 & -2.32 & -1.24 & 0.02593 \\
\hline 1.92 & 14.22 & 3.93 & 3.54 & 4.47 & 0.02659 & 0.67 & 0.34 & 1.17 & 0.02748 & -1.46 & -1.88 & -1.09 & 0.02452 \\
\hline 1.92 & 15.00 & 3.59 & 3.08 & 4.12 & 0.02980 & 0.55 & -0.12 & 1.10 & 0.03796 & -1.48 & -1.91 & -1.06 & 0.02320 \\
\hline 1.92 & 15.78 & 3.61 & 3.22 & 3.97 & 0.02402 & 0.24 & -0.16 & 0.72 & 0.02602 & \begin{tabular}{|l|}
-1.72 \\
\end{tabular} & -2.09 & -1.30 & 0.02504 \\
\hline 1.92 & 16.55 & 3.54 & 2.90 & 4.00 & 0.02994 & 0.56 & 0.04 & 1.08 & 0.03322 & -1.86 & -2.26 & -1.51 & 0.02223 \\
\hline 1.92 & 17.33 & 3.57 & 2.89 & 4.24 & 0.01306 & 0.98 & 0.38 & 1.45 & 0.01315 & -1.43 & -1.93 & -0.91 & 0.01145 \\
\hline 2.67 & 2.67 & 3.10 & 2.24 & 4.20 & 0.03594 & 0.21 & -0.20 & 0.88 & 0.01405 & \begin{tabular}{|l|}
-1.24 \\
\end{tabular} & -2.22 & -0.61 & 0.03044 \\
\hline 2.67 & 12.67 & 3.28 & 2.31 & 4.17 & 0.03010 & 0.58 & 0.05 & 1.05 & 0.01439 & -1.07 & -1.95 & -0.25 & 0.02824 \\
\hline 3.42 & 2.67 & 3.49 & 2.69 & 4.20 & 0.02023 & 0.30 & -0.20 & 0.81 & 0.01471 & -1.67 & -2.45 & -0.93 & 0.01775 \\
\hline 3.42 & 12.67 & 3.60 & 2.93 & 4.30 & 0.01964 & 0.62 & -0.16 & 1.14 & 0.01672 & \begin{tabular}{|l|}
-1.45 \\
\end{tabular} & -2.06 & -0.86 & 0.01698 \\
\hline
\end{tabular}




\begin{tabular}{|c|c|c|c|c|c|c|c|c|c|c|c|c|c|}
\hline 4.17 & 2.67 & 3.76 & 2.95 & 4.38 & 0.01635 & 0.31 & -0.20 & 0.91 & 0.01452 & -1.94 & -2.40 & -1.42 & 0.01427 \\
\hline 4.17 & 12.67 & 3.85 & 3.32 & 4.41 & 0.01520 & 0.66 & 0.14 & 1.14 & 0.01467 & -1.65 & -2.19 & -0.97 & 0.01499 \\
\hline 4.92 & 2.67 & 3.60 & 2.96 & 4.20 & 0.01793 & 0.28 & -0.22 & 0.78 & 0.01389 & -1.76 & -2.35 & -1.19 & 0.01891 \\
\hline 4.92 & 12.67 & 3.68 & 2.98 & 4.42 & 0.02110 & 0.63 & 0.01 & 1.15 & 0.01535 & -1.54 & -2.17 & -1.00 & 0.01641 \\
\hline 5.67 & 2.67 & 2.99 & 2.24 & 3.88 & 0.02473 & 0.17 & -0.25 & 0.69 & 0.01335 & -0.49 & -1.99 & 0.48 & 0.05430 \\
\hline 5.67 & 12.67 & 3.16 & 2.23 & 3.80 & 0.02226 & 0.53 & 0.01 & 1.13 & 0.01311 & -0.46 & -2.02 & 0.36 & 0.04424 \\
\hline 6.41 & 2.67 & 2.95 & 1.63 & 4.33 & 0.00474 & 0.06 & -1.02 & 1.42 & 0.00419 & 0.16 & -0.63 & 1.15 & 0.00333 \\
\hline 6.41 & 3.45 & 2.97 & 1.92 & 4.11 & 0.01646 & 0.06 & -0.82 & 1.20 & 0.01633 & 0.09 & -0.66 & 0.76 & 0.01348 \\
\hline 6.41 & 4.22 & 2.87 & 1.96 & 3.83 & 0.01537 & 0.17 & -0.79 & 1.18 & 0.01649 & -0.07 & -0.68 & 0.77 & 0.01223 \\
\hline 6.41 & 5.00 & 2.88 & 1.61 & 3.81 & 0.01455 & 0.30 & -0.62 & 1.70 & 0.01535 & -0.04 & -0.66 & 1.00 & 0.01332 \\
\hline 6.41 & 5.78 & 2.85 & 1.88 & 3.88 & 0.01486 & 0.49 & -0.48 & 1.40 & 0.01497 & -0.01 & -0.71 & 1.02 & 0.01320 \\
\hline 6.41 & 6.55 & 2.56 & 1.55 & 3.63 & 0.01654 & 0.47 & -0.39 & 1.59 & 0.01525 & 0.03 & -0.69 & 0.99 & 0.01568 \\
\hline 6.41 & 7.33 & 3.24 & 1.89 & 4.46 & 0.00426 & 0.26 & -0.71 & 1.26 & 0.00397 & 0.32 & -0.58 & 1.32 & 0.00358 \\
\hline 6.41 & 12.67 & 3.17 & 2.02 & 4.36 & 0.00338 & 0.49 & -0.68 & 1.69 & 0.00378 & 0.07 & -0.66 & 1.11 & 0.00284 \\
\hline 6.41 & 13.45 & 3.16 & 2.07 & 4.25 & 0.01456 & 0.51 & -0.40 & 1.58 & 0.01466 & 0.07 & -0.72 & 0.89 & 0.01236 \\
\hline 6.41 & 14.22 & 3.25 & 2.50 & 4.48 & 0.01423 & 0.76 & -0.08 & 1.76 & 0.01447 & 0.17 & -0.48 & 1.00 & 0.01117 \\
\hline 6.41 & 15.00 & 3.18 & 2.33 & 4.24 & 0.01463 & 0.91 & -0.01 & 1.75 & 0.01422 & 0.22 & -0.56 & 0.90 & 0.01081 \\
\hline 6.41 & 15.78 & 2.84 & 1.82 & 3.82 & 0.01414 & 0.89 & -0.03 & 1.79 & 0.01421 & 0.21 & -0.35 & 0.78 & 0.00955 \\
\hline 6.41 & 16.55 & 2.76 & 1.75 & 3.74 & 0.01275 & 0.57 & -0.27 & 1.52 & 0.01344 & 0.14 & -0.52 & 0.91 & 0.01014 \\
\hline 6.41 & 17.33 & 3.01 & 1.83 & 4.40 & 0.00436 & 0.81 & -0.46 & 1.99 & 0.00412 & 0.54 & -0.47 & 1.60 & 0.00377 \\
\hline 6.83 & 7.33 & 3.21 & 2.15 & 4.24 & 0.01433 & 0.23 & -0.74 & 1.17 & 0.01299 & 0.36 & -0.42 & 1.33 & 0.01264 \\
\hline 6.83 & 17.33 & 3.08 & 1.98 & 4.48 & 0.01515 & 0.82 & -0.31 & 1.99 & 0.01362 & 0.71 & -0.32 & 1.83 & 0.01302 \\
\hline 7.25 & 7.33 & 3.35 & 2.13 & 4.48 & 0.01638 & 0.23 & -0.59 & 1.31 & 343 & 0.44 & -0.57 & 1.70 & 0.01259 \\
\hline 7.25 & 17.33 & 3.22 & 2.02 & 4.44 & 0.01620 & 0.84 & -0.20 & 1.90 & 0.01467 & 0.83 & -0.21 & 1.65 & 0.01241 \\
\hline 7.66 & 7.33 & 3.51 & 2.63 & 4.92 & 0.01595 & 0.30 & -0.60 & 1.43 & 0.01409 & 0.49 & -0.49 & 1.19 & 0.01173 \\
\hline 7.66 & 17.33 & 3.36 & 2.15 & 4.69 & 0.01673 & 0.82 & -0.41 & 1.86 & 0.01430 & 0.80 & 0.02 & 1.67 & 0.01305 \\
\hline 8.08 & 7.33 & 3.43 & 2.42 & 4.51 & 0.01420 & 0.29 & -0.74 & 1.27 & 0.01403 & 0.40 & -0.59 & 1.34 & 0.01316 \\
\hline 8.08 & 17.33 & 3.40 & 2.09 & 4.60 & 0.01498 & 0.78 & -0.58 & 1.81 & 0.01420 & 0.89 & 0.16 & 1.90 & 0.01265 \\
\hline 8.50 & 7.33 & 3.03 & 2.20 & 4.07 & 0.01392 & 0.25 & -0.71 & 1.49 & 0.01304 & 0.35 & -0.42 & 1.11 & 0.01031 \\
\hline 8.50 & 17.33 & 2.97 & 1.87 & 4.28 & 0.01675 & 0.78 & -0.30 & 2.06 & 0.01415 & 0.81 & -0.26 & 1.75 & 0.01384 \\
\hline 8.85 & 2.67 & 2.80 & 1.72 & 3.81 & 0.00628 & -0.09 & -0.90 & 0.92 & 0.00560 & 0.49 & -0.41 & 1.21 & 0.00463 \\
\hline 8.85 & 3.45 & 2.72 & 1.68 & 3.68 & 0.01390 & 0.05 & -0.98 & 1.29 & 0.01502 & 0.24 & -0.34 & 0.94 & 0.01032 \\
\hline 8.85 & 4.22 & 2.67 & 1.65 & 3.65 & 0.01341 & 0.27 & -0.78 & 1.19 & 0.01301 & 0.12 & -0.57 & 0.99 & 0.01059 \\
\hline 8.85 & 5.00 & 2.41 & 1.30 & 3.29 & 0.01512 & 0.16 & -0.94 & 1.23 & 0.01510 & 0.09 & -0.59 & 0.80 & 0.01133 \\
\hline 8.85 & 5.78 & 2.85 & 1.89 & 3.93 & 0.01439 & 0.05 & -0.70 & 0.81 & 0.01399 & 0.25 & -0.63 & 1.11 & 0.01298 \\
\hline 8.85 & 6.55 & 2.87 & 2.06 & 3.88 & 0.01318 & 0.21 & -0.60 & 1.15 & 0.01315 & 0.34 & -0.57 & 1.24 & 0.01173 \\
\hline 8.85 & 7.33 & 2.89 & 1.90 & 3.97 & 0.01344 & 0.23 & -0.62 & 1.08 & 0.01169 & 0.34 & -0.30 & 1.11 & 0.01027 \\
\hline 8.85 & 12.67 & 2.98 & 1.82 & 4.18 & 0.00540 & 0.37 & -0.56 & 1.29 & 0.00522 & 0.13 & -0.51 & 0.94 & 0.00384 \\
\hline 8.85 & 13.45 & 3.02 & 2.12 & 4.14 & 0.01346 & 0.55 & -0.29 & 1.76 & 0.01365 & 0.22 & -0.69 & 1.00 & 0.00954 \\
\hline 8.85 & 14.22 & 2.75 & 1.67 & 3.81 & 0.01513 & 0.81 & -0.17 & 1.88 & 0.01330 & 0.26 & -0.47 & 0.98 & 0.00870 \\
\hline 8.85 & 15.00 & 2.61 & 1.48 & 3.56 & 0.01390 & 0.45 & -0.54 & 1.27 & 0.01249 & 0.27 & -0.45 & 0.98 & 0.01003 \\
\hline 8.85 & 15.78 & 2.74 & 1.93 & 3.91 & 0.01310 & 0.52 & -0.35 & 1.52 & 0.01398 & 0.42 & -0.35 & 1.14 & 0.01038 \\
\hline 8.85 & 16.55 & 2.77 & 1.31 & 4.06 & 0.01527 & 0.70 & -0.56 & 1.81 & 0.01411 & 0.70 & -0.21 & 1.54 & 0.01314 \\
\hline 8.85 & 17.33 & 2.80 & 1.83 & 3.96 & 0.01341 & 0.70 & -0.33 & 1.60 & 0.01288 & 0.73 & -0.05 & 1.54 & 0.01089 \\
\hline 8.92 & 2.67 & 2.65 & 1.54 & 3.98 & 0.00418 & 0.05 & -1.11 & 1.15 & 0.00386 & 0.25 & -0.54 & 1.20 & 0.00300 \\
\hline 8.92 & 3.45 & 2.64 & 1.51 & 3.88 & 0.01126 & 0.07 & -0.82 & 1.17 & 0.01109 & 0.16 & -0.60 & 0.89 & 0.00872 \\
\hline 8.92 & 4.22 & 2.60 & 1.71 & 3.64 & 0.01190 & 0.23 & -1.09 & 1.41 & 0.01201 & -0.06 & -0.82 & 0.83 & 0.00888 \\
\hline 8.92 & 5.00 & 2.43 & 1.52 & 3.51 & 0.01163 & 0.34 & -0.57 & 1.33 & 0.01073 & -0.03 & -0.68 & 0.84 & 0.00849 \\
\hline 8.92 & 5.78 & 2.51 & 1.42 & 3.62 & 0.01264 & 0.04 & -1.17 & 0.98 & 0.01170 & 0.05 & -0.80 & 0.70 & 0.00941 \\
\hline 8.92 & 6.55 & 2.78 & 1.82 & 3.87 & 0.01068 & 0.19 & -0.86 & 1.14 & 0.01103 & 0.15 & -0.60 & 1.28 & 0.00955 \\
\hline 8.92 & 7.33 & 2.85 & 1.63 & 4.07 & 0.00395 & 0.27 & -0.90 & 1.23 & 0.00380 & 0.28 & -0.53 & 1.20 & 0.00302 \\
\hline 8.92 & 12.67 & 2.90 & 1.84 & 4.05 & 0.00341 & 0.40 & -0.69 & 1.49 & 0.00367 & 0.12 & -0.59 & 1.00 & 0.00251 \\
\hline 8.92 & 13.45 & 2.86 & 1.95 & 3.84 & 0.01005 & 0.40 & -0.75 & 1.47 & 0.01146 & 0.12 & -0.56 & 0.99 & 0.00758 \\
\hline 8.92 & 14.22 & 2.96 & 1.76 & 3.86 & 0.01116 & 0.69 & -0.30 & 1.81 & 0.01191 & 0.19 & -0.39 & 0.91 & 0.00778 \\
\hline 8.92 & 15.00 & 2.50 & 1.50 & 3.65 & 0.01074 & 0.58 & -0.57 & 1.70 & 0.01193 & 0.24 & -0.73 & 1.01 & 0.00781 \\
\hline 8.92 & 15.78 & 2.56 & 1.46 & 3.76 & 0.01143 & 0.46 & -0.38 & 1.56 & 0.01134 & 0.23 & -0.47 & 1.02 & 0.00766 \\
\hline 8.92 & 16.55 & 2.68 & 1.49 & 3.85 & 0.01274 & 0.67 & -0.36 & 1.98 & 0.01180 & 0.44 & -0.46 & 1.46 & 0.01026 \\
\hline 8.92 & 17.33 & 2.67 & 1.59 & 4.08 & 0.00446 & 0.70 & -0.76 & 1.99 & 0.00393 & 0.67 & -0.34 & 1.66 & 0.00354 \\
\hline 8.94 & 2.67 & 2.84 & 2.15 & 3.60 & 0.02401 & -0.07 & -0.91 & 0.94 & 0.02744 & 0.51 & -0.09 & 1.02 & 0.01972 \\
\hline
\end{tabular}




\begin{tabular}{|c|c|c|c|c|c|c|c|c|c|c|c|c|c|}
\hline 8.94 & 12.67 & 3.01 & 2.22 & 4.12 & 0.02454 & 0.43 & -0.46 & 1.12 & 0.02044 & 0.21 & -0.23 & 0.78 & 0.01407 \\
\hline 9.02 & 2.67 & 2.72 & 2.16 & 3.27 & 0.03769 & 0.09 & -0.55 & 0.95 & 0.04428 & 0.49 & 0.06 & 1.04 & 0.03440 \\
\hline 9.02 & 12.67 & 2.99 & 2.33 & 3.89 & 0.03888 & 0.31 & -0.48 & 0.82 & 0.03709 & 0.09 & -0.49 & 0.58 & 0.02405 \\
\hline 9.10 & 2.67 & 2.96 & 2.00 & 4.06 & 0.03366 & -0.11 & -0.91 & 0.56 & 0.02815 & 0.58 & 0.02 & 1.28 & 0.02273 \\
\hline 9.10 & 12.67 & 3.19 & 2.52 & 3.86 & 0.01924 & 0.45 & -0.48 & 1.08 & 0.02164 & 0.22 & -0.44 & 0.85 & 0.02073 \\
\hline 9.19 & 2.67 & 2.66 & 2.10 & 3.29 & 0.03323 & -0.20 & -0.84 & 0.67 & 0.03831 & 0.47 & 0.04 & 1.20 & 0.02954 \\
\hline 9.19 & 12.67 & 2.79 & 2.01 & 3.25 & 0.03221 & 0.39 & -0.56 & 0.98 & 0.04427 & 0.04 & -0.39 & 0.50 & 0.02641 \\
\hline 9.27 & 2.67 & 2.57 & 1.75 & 3.74 & 0.02568 & -0.10 & -0.90 & 0.77 & 0.02435 & 0.39 & -0.48 & 1.31 & 0.02394 \\
\hline 9.27 & 12.67 & 2.74 & 1.73 & 3.59 & 0.02563 & 0.31 & -0.52 & 1.09 & 0.02200 & 0.04 & -0.53 & 0.54 & 0.01388 \\
\hline 9.35 & 2.67 & 2.52 & 1.47 & 3.62 & 0.00638 & -0.08 & -0.85 & 0.87 & 0.00550 & 0.41 & -0.39 & 1.20 & 0.00463 \\
\hline 9.35 & 3.45 & 2.43 & 1.56 & 3.23 & 0.01250 & 0.02 & -0.88 & 0.86 & 0.01246 & 0.24 & -0.49 & 0.93 & 0.01067 \\
\hline 9.35 & 4.22 & 2.38 & 1.35 & 3.25 & 0.01498 & 0.18 & -0.75 & 1.82 & 0.01614 & 0.04 & -0.60 & 0.80 & 0.01100 \\
\hline 9.35 & 5.00 & 2.16 & 1.21 & 3.01 & 0.01374 & 0.29 & -0.61 & 1.15 & 0.01379 & 0.01 & -0.53 & 0.77 & 0.00973 \\
\hline 9.35 & 5.78 & 2.40 & 1.09 & 3.59 & 0.01633 & 0.02 & -0.89 & 1.19 & 0.01459 & 0.01 & -0.66 & 0.92 & 0.01051 \\
\hline 9.35 & 6.55 & 2.69 & 1.66 & 3.68 & 0.01405 & 0.24 & -0.82 & 1.02 & 0.01321 & 0.19 & -0.68 & 1.16 & 0.01230 \\
\hline 9.35 & 7.33 & 2.68 & 1.58 & 3.95 & 0.01151 & 0.28 & -0.66 & 1.26 & 0.01054 & 0.24 & -0.52 & 1.00 & 0.00844 \\
\hline 9.35 & 12.67 & 2.78 & 1.70 & 4.15 & 0.00555 & 0.43 & -0.54 & 1.31 & 0.00550 & 0.10 & -0.52 & 0.85 & 0.00377 \\
\hline 9.35 & 13.45 & 2.76 & 1.94 & 3.71 & 0.01240 & 0.47 & -0.43 & 1.35 & 0.01256 & 0.14 & -0.47 & 0.98 & 0.00904 \\
\hline 9.35 & 14.22 & 2.84 & 1.93 & 3.83 & 0.01347 & 0.61 & -0.56 & 1.44 & 0.01380 & 0.16 & -0.43 & 0.78 & 0.00877 \\
\hline 9.35 & 15.00 & 2.41 & 1.50 & 3.24 & 0.01314 & 0.58 & -0.39 & 1.89 & 0.01454 & 0.23 & -0.29 & 0.88 & 0.00860 \\
\hline 9.35 & 15.78 & 2.46 & 1.58 & 3.49 & 0.01274 & 0.47 & -0.74 & 1.40 & 0.01366 & 0.24 & -0.42 & 1.02 & 0.00925 \\
\hline 9.35 & 16.55 & 2.71 & 1.28 & 3.75 & 0.01469 & 0.76 & -0.16 & 1.92 & 0.01416 & 0.56 & -0.31 & 1.37 & 0.01342 \\
\hline 9.35 & 17.33 & 2.70 & 1.46 & 4.08 & 0.01197 & 0.78 & -0.37 & 1.69 & 0.01145 & 0.74 & -0.08 & 1.63 & 0.01001 \\
\hline 9.38 & 2.67 & 2.50 & 1.47 & 3.47 & 0.01681 & -0.07 & -0.93 & 1.01 & 0.01527 & 0.44 & -0.22 & 1.20 & 0.01069 \\
\hline 9.38 & 12.67 & 2.65 & 1.82 & 3.67 & 0.01260 & 0.38 & -0.76 & 1.25 & 0.01314 & 0.25 & -0.27 & 0.99 & 0.00839 \\
\hline 9.44 & 7.33 & 2.74 & 1.82 & 3.63 & 0.02386 & 0.19 & -0.68 & 1.11 & 0.02586 & 0.30 & -0.22 & 1.04 & 0.01678 \\
\hline 9.44 & 17.33 & 2.54 & 1.49 & 3.35 & 0.02807 & 0.66 & 0.01 & 1.52 & 0.02546 & 0.59 & 0.02 & 1.20 & 0.01995 \\
\hline 9.52 & 7.33 & 2.44 & 1.67 & 3.27 & 0.04391 & 0.20 & -0.62 & 0.85 & 0.03706 & 0.30 & 0.08 & 0.68 & 0.01511 \\
\hline 9.52 & 17.33 & 2.51 & 1.66 & 3.32 & 0.04333 & 0.71 & -0.05 & 1.48 & 0.04178 & 0.87 & 0.37 & 1.64 & 0.02903 \\
\hline 9.60 & 7.33 & 2.59 & 1.86 & 3.45 & 0.02309 & 0.24 & -0.50 & 1.01 & 0.02418 & 0.48 & -0.09 & 1.17 & 0.02256 \\
\hline 9.60 & 17.33 & 2.56 & 1.44 & 3.40 & 0.02629 & 0.72 & -0.08 & 1.49 & 0.02313 & 0.91 & 0.26 & 1.76 & 0.02167 \\
\hline 9.69 & 7.33 & 2.35 & 1.60 & 3.02 & 0.03918 & 0.15 & -0.62 & 0.93 & 0.04207 & 0.32 & 0.01 & 0.84 & 0.02350 \\
\hline 9.69 & 17.33 & 2.46 & 1.48 & 3.43 & 0.05408 & 0.77 & -0.01 & 1.33 & 0.03800 & 0.82 & 0.36 & 1.45 & 0.02556 \\
\hline 9.77 & 7.33 & 2.54 & 1.55 & 3.60 & 0.02548 & 0.30 & -0.43 & 1.14 & 0.02466 & 0.42 & -0.24 & 0.99 & 0.01947 \\
\hline 9.77 & 17.33 & 2.53 & 1.59 & 3.71 & 0.02931 & 0.82 & 0.03 & 1.56 & 0.02220 & 0.92 & 0.29 & 1.72 & 0.02150 \\
\hline 9.84 & 2.67 & 2.46 & 1.30 & 3.62 & 0.01602 & -0.03 & -1.02 & 0.90 & 0.01467 & 0.45 & -0.24 & 1.07 & 0.00934 \\
\hline 9.84 & 12.67 & 2.68 & 1.73 & 3.78 & 0.01311 & 0.39 & -0.69 & 1.22 & 0.01331 & 0.29 & -0.30 & 0.86 & 0.00767 \\
\hline 9.85 & 2.67 & 2.41 & 1.55 & 3.51 & 0.00763 & -0.14 & -0.92 & 0.78 & 0.00651 & 0.62 & -0.07 & 1.25 & 0.00471 \\
\hline 9.85 & 3.45 & 2.38 & 1.37 & 3.49 & 0.01540 & -0.08 & -1.00 & 0.81 & 0.01430 & 0.49 & -0.20 & 1.29 & 0.01150 \\
\hline 9.85 & 4.22 & 2.29 & 1.38 & 3.43 & 0.01536 & 0.10 & -1.00 & 1.18 & 0.01472 & 0.28 & -0.32 & 0.89 & 0.00930 \\
\hline 9.85 & 5.00 & 2.12 & 0.87 & 3.04 & 0.01619 & 0.21 & -0.82 & 1.01 & 0.01469 & 0.19 & -0.34 & 0.91 & 0.00950 \\
\hline 9.85 & 5.78 & 2.46 & 1.46 & 3.43 & 0.01439 & 0.01 & -1.04 & 1.06 & 0.01665 & 0.18 & -0.43 & 0.81 & 0.01009 \\
\hline 9.85 & 6.55 & 2.52 & 1.55 & 3.56 & 0.01542 & 0.21 & -0.58 & 1.20 & 0.01343 & 0.35 & -0.42 & 1.32 & 0.01227 \\
\hline 9.85 & 7.33 & 2.50 & 1.58 & 3.69 & 0.01123 & 0.19 & -0.68 & 1.17 & 0.01147 & 0.42 & -0.11 & 1.22 & 0.00729 \\
\hline 9.85 & 12.67 & 2.59 & 1.56 & 3.56 & 0.00569 & 0.44 & -0.45 & 1.27 & 0.00551 & 0.25 & -0.25 & 1.02 & 0.00357 \\
\hline 9.85 & 13.45 & 2.55 & 1.65 & 3.53 & 0.01394 & 0.45 & -0.60 & 1.38 & 0.01358 & 0.29 & -0.25 & 1.03 & 0.00922 \\
\hline 9.85 & 14.22 & 2.50 & 1.53 & 3.59 & 0.01362 & 0.70 & -0.25 & 1.38 & 0.01272 & 0.29 & -0.38 & 0.91 & 0.00960 \\
\hline 9.85 & 15.00 & 2.28 & 1.44 & 3.22 & 0.01346 & 0.59 & -0.39 & 1.69 & 0.01455 & 0.37 & -0.30 & 1.06 & 0.00943 \\
\hline 9.85 & 15.78 & 2.41 & 1.49 & 3.52 & 0.01438 & 0.60 & -0.46 & 1.72 & 0.01561 & 0.52 & 0.00 & 1.28 & 0.00996 \\
\hline 9.85 & 16.55 & 2.45 & 1.59 & 3.63 & 0.01600 & 0.72 & -0.19 & 1.67 & 0.01456 & 0.77 & -0.08 & 1.47 & 0.01252 \\
\hline 9.85 & 17.33 & 2.44 & 1.19 & 3.85 & 0.01247 & 0.66 & -0.34 & 1.67 & 0.01147 & 0.81 & -0.03 & 1.67 & 0.01021 \\
\hline 9.93 & 2.67 & 2.47 & 1.64 & 3.63 & 0.02930 & -0.14 & -0.76 & 0.55 & 0.02227 & 0.57 & -0.07 & 1.21 & 0.01982 \\
\hline 9.93 & 12.67 & 2.47 & 1.77 & 3.31 & 0.02052 & 0.41 & -0.58 & 1.27 & 0.02131 & 0.23 & -0.14 & 0.94 & 0.01248 \\
\hline 10.00 & 2.67 & 2.58 & 1.81 & 3.18 & 0.04253 & -0.20 & -0.84 & 0.26 & 0.02862 & 0.80 & 0.22 & 1.60 & 0.03437 \\
\hline 10.00 & 12.67 & 2.77 & 2.07 & 3.75 & 0.03861 & 0.44 & -0.14 & 1.31 & 0.03154 & 0.70 & 0.16 & 1.38 & 0.03698 \\
\hline 10.07 & 2.67 & 2.76 & 1.61 & 3.95 & 0.03281 & -0.20 & -1.07 & 0.42 & 0.02439 & 0.93 & 0.34 & 1.59 & 0.02078 \\
\hline 10.07 & 12.67 & 2.72 & 2.00 & 3.63 & 0.02224 & 0.44 & -0.49 & 1.18 & 0.02316 & 0.58 & 0.18 & 1.22 & 0.01690 \\
\hline 10.13 & 2.67 & 2.79 & 2.22 & 3.70 & 0.04538 & -0.24 & -1.12 & 0.58 & 0.05702 & 1.00 & 0.52 & 1.53 & 0.03051 \\
\hline 10.13 & 12.67 & 2.69 & 2.08 & 3.53 & 0.03971 & 0.37 & -0.36 & 1.02 & 0.03578 & 0.58 & 0.21 & 0.94 & 0.02119 \\
\hline
\end{tabular}




\begin{tabular}{|c|c|c|c|c|c|c|c|c|c|c|c|c|c|}
\hline 10.2 & 2.67 & 2.82 & 2.05 & 3.71 & 0.02553 & -0.10 & -0.86 & 0.75 & 0.02677 & 1.00 & 0.33 & 1.59 & 0.01999 \\
\hline 10.2 & 12.67 & 2.68 & 1.85 & 3.53 & 0.02578 & 0.51 & -0.46 & 1.41 & 0.02574 & 0.72 & 0.28 & 1.29 & 0.01665 \\
\hline 10.27 & 2.67 & 2.76 & 1.68 & 3.91 & 0.00631 & -0.14 & -1.12 & 1.02 & 0.00554 & 0.96 & 0.22 & 1.77 & 0.00414 \\
\hline 10.27 & 3.45 & 2.68 & 1.72 & 3.73 & 0.01447 & -0.08 & -0.99 & 0.78 & 0.01297 & 0.86 & 0.21 & 1.50 & 0.00989 \\
\hline 10.27 & 4.22 & 2.66 & 1.64 & 3.85 & 0.01639 & 0.19 & -0.90 & 1.40 & 0.01512 & 0.58 & -0.14 & 1.53 & 0.01108 \\
\hline 10.27 & 5.00 & 2.23 & 1.33 & 3.32 & 0.01307 & 0.19 & -0.86 & 1.19 & 0.01183 & 0.44 & -0.12 & 1.08 & 0.00824 \\
\hline 10.27 & 5.78 & 2.37 & 1.58 & 3.45 & 0.01435 & -0.04 & -0.93 & 0.86 & 0.01350 & 0.50 & -0.06 & 1.27 & 0.00891 \\
\hline 10.27 & 6.55 & 2.72 & 1.79 & 3.82 & 0.01340 & 0.08 & -0.74 & 0.99 & 0.01289 & 0.62 & -0.12 & 1.48 & 0.01083 \\
\hline 10.27 & 7.33 & 2.77 & 1.75 & 3.86 & 0.01251 & 0.21 & -0.61 & 1.29 & 0.01166 & 0.74 & 0.11 & 1.46 & 0.00871 \\
\hline 10.27 & 12.67 & 2.80 & 1.90 & 3.86 & 0.00497 & 0.48 & -0.53 & 1.38 & 0.00491 & 0.65 & 0.06 & 1.33 & 0.00339 \\
\hline 10.27 & 13.45 & 2.81 & 1.87 & 3.75 & 0.01241 & 0.49 & -0.46 & 1.39 & 0.01188 & 0.67 & 0.00 & 1.38 & 0.00830 \\
\hline 10.27 & 14.22 & 2.62 & 1.65 & 3.67 & 0.01496 & 0.66 & -0.12 & 1.63 & 0.01263 & 0.64 & -0.05 & 1.35 & 0.00998 \\
\hline 10.27 & 15.00 & 2.48 & 1.39 & 3.45 & 0.01237 & 0.34 & -0.67 & 1.20 & 0.01152 & 0.69 & 0.07 & 1.42 & 0.00834 \\
\hline 10.27 & 15.78 & 2.70 & 1.70 & 3.62 & 0.01427 & 0.38 & -0.64 & 1.56 & 0.01369 & 0.82 & 0.09 & 1.55 & 0.00959 \\
\hline 10.27 & 16.55 & 2.72 & 1.49 & 3.87 & 0.01417 & 0.57 & -0.28 & 1.64 & 0.01302 & 1.02 & 0.45 & 1.94 & 0.01028 \\
\hline 10.27 & 17.33 & 2.66 & 1.62 & 3.69 & 0.01228 & 0.61 & -0.33 & 1.45 & 0.01112 & 1.17 & 0.28 & 2.02 & 0.00985 \\
\hline 10.29 & 2.67 & 2.78 & 1.38 & 4.23 & 0.02250 & -0.04 & -0.91 & 1.02 & 0.01384 & 0.75 & -0.14 & 1.54 & 0.01364 \\
\hline 10.29 & 12.67 & 2.88 & 1.74 & 3.97 & 0.01765 & 0.40 & -0.58 & 1.30 & 0.01306 & 0.56 & -0.24 & 1.44 & 0.01268 \\
\hline 10.75 & 2.67 & 3.39 & 2.28 & 4.46 & 0.01823 & -0.12 & -1.02 & 0.78 & 0.01582 & 1.00 & 0.11 & 1.51 & 0.01104 \\
\hline 10.75 & 12.67 & 3.33 & 2.30 & 4.45 & 0.01364 & 0.50 & -0.55 & 1.25 & 0.01420 & 0.78 & 0.03 & 1.48 & 0.01071 \\
\hline 10.86 & 2.67 & 3.16 & 2.04 & 4.37 & 0.00872 & -0.09 & -1.09 & 0.91 & 0.00783 & 1.04 & 0.29 & 1.63 & 0.00573 \\
\hline 10.86 & 3.45 & 3.13 & 2.15 & 4.26 & 0.01485 & -0.08 & -0.99 & 0.88 & 0.01452 & 1.00 & 0.32 & 1.88 & 0.01179 \\
\hline 10.86 & 4.22 & 3.07 & 2.27 & 4.11 & 0.01523 & 0.13 & -0.87 & 1.18 & 0.01321 & 0.80 & 0.13 & 1.62 & 0.01064 \\
\hline 10.86 & 5.00 & 2.73 & 1.79 & 4.24 & 0.01621 & 0.23 & -0.55 & 1.29 & 0.01382 & 0.63 & 0.01 & 1.26 & 0.01007 \\
\hline 10.86 & 5.78 & 2.95 & 2.14 & 4.19 & 0.01453 & 0.02 & -0.81 & 0.90 & 0.01381 & 0.74 & 0.04 & 1.55 & 0.01233 \\
\hline 10.86 & 6.55 & 3.09 & 2.29 & 4.05 & 0.01350 & 0.16 & -0.70 & 1.05 & 0.01334 & 0.76 & 0.02 & 1.59 & 0.01099 \\
\hline 10.86 & 7.33 & 3.11 & 1.98 & 4.34 & 0.01432 & 0.15 & -0.68 & 1.09 & 0.01167 & 0.82 & 0.13 & 1.68 & 0.01100 \\
\hline 10.86 & 12.67 & 3.12 & 2.15 & 4.25 & 0.00729 & 0.46 & -0.40 & 1.46 & 0.00700 & 0.77 & 0.16 & 1.40 & 0.00529 \\
\hline 10.86 & 13.45 & 3.10 & 2.09 & 4.08 & 0.01398 & 0.47 & -0.35 & 1.32 & 0.01223 & 0.79 & 0.17 & 1.39 & 0.00927 \\
\hline 10.86 & 14.22 & 3.11 & 2.05 & 4.13 & 0.01358 & 0.65 & -0.33 & 1.49 & 0.01229 & 0.83 & 0.10 & 1.52 & 0.01049 \\
\hline 10.86 & 15.00 & 2.81 & 1.80 & 3.85 & 0.01693 & 0.48 & -0.34 & 1.32 & 0.01439 & 0.82 & 0.23 & 1.54 & 0.01027 \\
\hline 10.86 & 15.78 & 3.22 & 2.17 & 4.23 & 0.01438 & 0.51 & -0.39 & 1.59 & 0.01301 & 1.02 & 0.43 & 1.65 & 0.00939 \\
\hline 10.86 & 16.55 & 3.26 & 2.37 & 4.29 & 0.01417 & 0.66 & -0.45 & 1.57 & 0.01438 & 1.31 & 0.45 & 2.25 & 0.01100 \\
\hline 10.86 & 17.33 & 3.20 & 2.03 & 4.30 & 0.01344 & 0.66 & -0.63 & 1.61 & 0.01375 & 1.40 & 0.71 & 2.48 & 103 \\
\hline 10.92 & 2.67 & 3.36 & 2.72 & 3.81 & 0.04263 & -0.09 & -0.71 & 0.44 & 0.03845 & 1.09 & 0.68 & 1.76 & 0.03523 \\
\hline 10.92 & 12.67 & 3.30 & 2.48 & 4.26 & 0.07760 & 0.39 & -0.52 & 1.17 & 0.06146 & 0.73 & 0.25 & 1.18 & 0.03828 \\
\hline 10.97 & 2.67 & 3.39 & 2.56 & 4.16 & 0.06651 & 0.05 & -0.73 & 0.93 & 0.05958 & 1.13 & 0.42 & 1.47 & 0.04615 \\
\hline 10.97 & 12.67 & 3.16 & 2.57 & 3.59 & 0.04332 & 0.35 & -0.16 & 0.71 & 0.04643 & 0.84 & 0.44 & 1.21 & 0.03484 \\
\hline 11.03 & 2.67 & 3.10 & 2.49 & 3.79 & 0.06201 & -0.18 & -0.64 & 0.51 & 0.04467 & 1.11 & 0.58 & 1.70 & 0.05829 \\
\hline 11.03 & 12.67 & 3.19 & 2.50 & 3.91 & 0.05102 & 0.62 & 0.01 & 1.32 & 0.05110 & 0.84 & 0.36 & 1.16 & 0.03290 \\
\hline 11.09 & 2.67 & 3.27 & 2.67 & 4.12 & 0.05124 & -0.21 & -0.89 & 0.47 & 0.05253 & 1.25 & 0.56 & 1.81 & 0.04908 \\
\hline 11.09 & 12.67 & 3.16 & 2.54 & 3.70 & 0.04651 & 0.48 & -0.40 & 1.14 & 0.05518 & 0.51 & 0.20 & 0.98 & 0.02539 \\
\hline 11.14 & 2.67 & 2.95 & 2.41 & 3.43 & 0.05662 & -0.24 & -0.93 & 0.50 & 0.07012 & 1.05 & 0.52 & 1.58 & 0.05488 \\
\hline 11.14 & 12.67 & 3.10 & 2.59 & 3.46 & 0.04036 & 0.47 & -0.07 & 0.96 & 0.04866 & 0.62 & 0.25 & 1.14 & 0.03805 \\
\hline 11.20 & 2.67 & 3.16 & 2.09 & 4.40 & 0.00555 & -0.14 & -1.02 & 0.79 & 0.00493 & 0.99 & 0.19 & 1.64 & 0.00395 \\
\hline 11.20 & 3.45 & 3.13 & 2.06 & 4.16 & 0.01393 & -0.03 & -0.92 & 1.02 & 0.01293 & 0.99 & 0.19 & 1.70 & 0.01090 \\
\hline 11.20 & 4.22 & 3.20 & 2.31 & 4.18 & 0.01440 & -0.03 & -0.94 & 0.80 & 0.01392 & 0.94 & 0.29 & 1.58 & 0.01053 \\
\hline 11.20 & 5.00 & 2.98 & 2.00 & 4.07 & 0.01448 & 0.15 & -0.74 & 1.00 & 0.01242 & 0.60 & 0.05 & 1.18 & 0.00872 \\
\hline 11.20 & 5.78 & 2.69 & 1.88 & 3.86 & 0.01592 & -0.11 & -0.92 & 0.67 & 0.01208 & 0.54 & 0.00 & 1.45 & 0.01095 \\
\hline 11.20 & 6.55 & 3.11 & 2.08 & 4.08 & 0.01281 & -0.06 & -0.93 & 1.02 & 0.01315 & 0.74 & 0.09 & 1.54 & 0.01003 \\
\hline 11.20 & 7.33 & 3.00 & 2.16 & 3.96 & 0.01195 & 0.15 & -0.56 & 0.92 & 0.01015 & 0.72 & 0.14 & 1.55 & 0.00826 \\
\hline 11.20 & 12.67 & 3.23 & 2.22 & 4.49 & 0.00515 & 0.46 & -0.66 & 1.37 & 0.00450 & 0.75 & 0.13 & 1.48 & 0.00324 \\
\hline 11.20 & 13.45 & 3.25 & 2.11 & 4.21 & 0.01276 & 0.45 & -0.38 & 1.37 & 0.01113 & 0.76 & 0.12 & 1.45 & 0.00908 \\
\hline 11.20 & 14.22 & 3.18 & 2.37 & 4.18 & 0.01340 & 0.47 & -0.40 & 1.35 & 0.01260 & 0.70 & 0.01 & 1.47 & 0.00870 \\
\hline 11.20 & 15.00 & 2.82 & 1.90 & 3.99 & 0.01388 & 0.58 & -0.63 & 1.38 & 0.01240 & 0.76 & 0.25 & 1.32 & 0.00777 \\
\hline 11.20 & 15.78 & 3.04 & 1.91 & 4.08 & 0.01459 & 0.24 & -0.68 & 1.29 & 0.01394 & 0.84 & 0.20 & 1.69 & 0.00954 \\
\hline 11.20 & 16.55 & 3.28 & 2.13 & 4.20 & 0.01336 & 0.57 & -0.44 & 1.32 & 0.01135 & 1.12 & 0.34 & 1.75 & 0.00976 \\
\hline 11.20 & 17.33 & 3.22 & 2.33 & 4.29 & 0.01187 & 0.63 & -0.30 & 1.49 & 0.01110 & 1.20 & 0.50 & 1.98 & 0.00944 \\
\hline 11.21 & 2.67 & 3.36 & 2.03 & 4.52 & 0.01718 & -0.11 & -1.01 & 0.81 & 0.01403 & 1.01 & 0.25 & 1.54 & 0.01070 \\
\hline
\end{tabular}




\begin{tabular}{|c|c|c|c|c|c|c|c|c|c|c|c|c|c|}
\hline 11.21 & 12.67 & 3.27 & 2.29 & 4.26 & 0.01497 & 0.40 & -0.41 & 1.25 & 0.01400 & 0.76 & 0.08 & 1.54 & 0.01093 \\
\hline 11.28 & 7.33 & 2.99 & 2.53 & 3.52 & 0.04016 & 0.14 & -0.35 & 0.85 & 0.05456 & 0.75 & 0.41 & 1.13 & 0.03255 \\
\hline 11.28 & 17.33 & 3.06 & 2.52 & 3.53 & 0.03429 & 0.47 & 0.05 & 1.05 & 0.03441 & 1.20 & 0.80 & 1.59 & 0.02942 \\
\hline 11.37 & 7.33 & 3.24 & 2.61 & 3.92 & 0.06822 & 0.17 & -0.46 & 1.01 & 0.06258 & 0.80 & 0.51 & 1.20 & 0.03835 \\
\hline 11.37 & 17.33 & 3.45 & 2.84 & 3.89 & 0.04659 & 0.71 & 0.21 & 1.18 & 0.04191 & 1.30 & 0.83 & 1.83 & 0.03672 \\
\hline 11.45 & 7.33 & 3.04 & 2.52 & 3.63 & 0.03727 & 0.17 & -0.54 & 0.89 & 0.05857 & 0.79 & 0.34 & 1.10 & 0.03174 \\
\hline 11.45 & 17.33 & 3.27 & 2.76 & 3.77 & 0.04284 & 0.64 & -0.14 & 1.21 & 0.04742 & 1.20 & 0.84 & 1.77 & 0.03933 \\
\hline 11.53 & 7.33 & 3.09 & 2.43 & 3.70 & 0.05015 & 0.29 & -0.32 & 0.70 & 0.03403 & 0.66 & 0.24 & 1.36 & 0.05285 \\
\hline 11.53 & 17.33 & 3.09 & 2.51 & 3.70 & 0.04597 & 0.65 & 0.16 & 1.12 & 0.03858 & 1.09 & 0.36 & 1.53 & 0.03963 \\
\hline 11.62 & 7.33 & 2.92 & 2.48 & 3.55 & 0.05034 & -0.11 & -0.56 & 0.69 & 0.04799 & 0.60 & 0.23 & 1.36 & 0.05311 \\
\hline 11.62 & 17.33 & 3.05 & 2.35 & 3.49 & 0.04972 & 0.59 & -0.30 & 1.07 & 0.05054 & 0.98 & 0.35 & 1.47 & 0.05504 \\
\hline 11.67 & 2.67 & 3.24 & 2.12 & 4.32 & 0.00409 & -0.07 & -0.97 & 0.91 & 0.00359 & 0.96 & 0.23 & 1.66 & 0.00274 \\
\hline 11.67 & 3.45 & 3.28 & 2.28 & 4.26 & 0.01212 & -0.05 & -0.98 & 1.09 & 0.01034 & 0.85 & 0.09 & 1.43 & 0.00849 \\
\hline 11.67 & 4.22 & 3.18 & 2.08 & 4.43 & 0.01426 & 0.13 & -0.96 & 1.08 & 0.01123 & 0.65 & 0.01 & 1.35 & 0.00858 \\
\hline 11.67 & 5.00 & 2.68 & 1.79 & 4.41 & 0.01289 & 0.08 & -1.02 & 1.33 & 0.01148 & 0.55 & 0.02 & 1.44 & 0.00832 \\
\hline 11.67 & 5.78 & 2.99 & 1.97 & 4.19 & 0.01200 & -0.02 & -1.04 & 1.03 & 0.01107 & 0.66 & 0.04 & 1.62 & 0.00846 \\
\hline 11.67 & 6.55 & 3.14 & 2.15 & 4.41 & 0.01350 & 0.15 & -0.89 & 1.18 & 0.01217 & 0.81 & 0.06 & 1.67 & 0.00968 \\
\hline 11.67 & 7.33 & 3.16 & 2.07 & 4.23 & 0.00408 & 0.23 & -0.64 & 1.26 & 0.00360 & 0.85 & 0.12 & 1.73 & 0.00298 \\
\hline 11.67 & 12.67 & 3.21 & 2.21 & 4.52 & 0.00377 & 0.43 & -0.57 & 1.48 & 0.00352 & 0.80 & 0.15 & 1.63 & 0.00259 \\
\hline 11.67 & 13.45 & 3.23 & 2.08 & 4.28 & 0.01155 & 0.55 & -0.66 & 1.41 & 0.01032 & 0.88 & 0.26 & 1.65 & 0.00763 \\
\hline 11.67 & 14.22 & 3.06 & 1.94 & 4.16 & 0.01275 & 0.67 & -0.25 & 1.48 & 0.01003 & 0.89 & 0.26 & 1.64 & 0.00769 \\
\hline 11.67 & 15.00 & 2.97 & 1.90 & 3.98 & 0.01198 & 0.32 & -0.58 & 1.29 & 0.01055 & 0.86 & 0.16 & 1.42 & 0.00700 \\
\hline 11.67 & 15.78 & 3.26 & 2.20 & 4.32 & 0.01173 & 0.52 & -0.40 & 1.50 & 0.01073 & 0.96 & 0.32 & 1.90 & 0.00817 \\
\hline 11.67 & 16.55 & 3.24 & 2.09 & 4.23 & 0.01169 & 0.73 & -0.15 & 1.46 & 0.01046 & 1.18 & 0.41 & 1.92 & 0.00942 \\
\hline 11.67 & 17.33 & 3.22 & 1.93 & 4.26 & 0.00403 & 0.71 & -0.65 & 1.59 & 0.00370 & 1.24 & 0.41 & 2.10 & 0.00300 \\
\hline 11.70 & 2.67 & 3.19 & 2.20 & 4.27 & 0.00845 & -0.07 & -0.83 & 0.82 & 0.00728 & 1.04 & 0.28 & 1.72 & 0.00586 \\
\hline 11.70 & 3.45 & 3.35 & 2.26 & 4.50 & 0.01783 & -0.05 & -0.98 & 0.89 & 0.01395 & 0.96 & 0.12 & 1.65 & 0.01327 \\
\hline 11.70 & 4.22 & 3.24 & 2.21 & 4.47 & 0.01766 & 0.09 & -0.73 & 0.92 & 0.01378 & 0.77 & 0.18 & 1.55 & 0.01204 \\
\hline 11.70 & 5.00 & 2.73 & 1.85 & 3.98 & 0.01759 & 0.19 & -0.85 & 1.07 & 0.01446 & 0.57 & -0.07 & 1.31 & 0.00986 \\
\hline 11.70 & 5.78 & 2.90 & 1.85 & 3.90 & 0.01631 & -0.01 & -0.92 & 0.92 & 0.01438 & 0.60 & 0.07 & 1.24 & 0.01070 \\
\hline 11.70 & 6.55 & 3.08 & 2.13 & 3.99 & 0.01433 & 0.13 & -0.75 & 1.00 & 0.01360 & 0.76 & 0.17 & 1.55 & 0.01061 \\
\hline 11.70 & 7.33 & 2.97 & 2.01 & 4.05 & 0.01154 & 0.11 & -0.90 & 1.07 & 0.01030 & 0.74 & 0.05 & 1.61 & 0.00893 \\
\hline 11.70 & 12.67 & 3.22 & 2.23 & 4.30 & 0.00760 & 0.42 & -0.87 & 1.38 & 0.00714 & 0.81 & 0.24 & 1.52 & 0.00522 \\
\hline 11.70 & 13.45 & 3.42 & 2.54 & 4.69 & 0.01580 & 0.52 & -0.25 & 1.59 & 0.01294 & 0.89 & 0.18 & 1.61 & 0.01162 \\
\hline 11.70 & 14.22 & 3.09 & 2.07 & 4.04 & 0.01572 & 0.72 & -0.22 & 1.57 & 0.01281 & 0.80 & 0.28 & 1.38 & 0.00986 \\
\hline 11.70 & 15.00 & 2.75 & 1.77 & 3.63 & 0.01489 & 0.38 & -0.49 & 1.25 & 0.01323 & 0.83 & 0.32 & 1.37 & 0.00929 \\
\hline 11.70 & 15.78 & 3.18 & 2.35 & 4.18 & 0.01425 & 0.48 & -0.40 & 1.69 & 0.01446 & 0.99 & 0.42 & 1.70 & 0.01132 \\
\hline 11.70 & 16.55 & 3.12 & 1.93 & 4.10 & 0.01441 & 0.67 & -0.35 & 1.58 & 0.01488 & 1.20 & 0.55 & 1.84 & 0.01056 \\
\hline 11.70 & 17.33 & 3.12 & 2.12 & 4.18 & 0.01074 & 0.65 & -0.30 & 1.51 & 0.00966 & 1.18 & 0.27 & 1.89 & 0.00755 \\
\hline 12.09 & 7.33 & 3.16 & 2.33 & 4.46 & 0.01989 & 0.23 & -0.51 & 1.08 & 0.01713 & 0.98 & 0.34 & 1.68 & 629 \\
\hline 12.09 & 17.33 & 3.15 & 2.17 & 4.47 & 0.02151 & 0.68 & -0.54 & 1.58 & 0.01851 & 1.38 & 0.65 & 2.09 & 0.01401 \\
\hline 12.50 & 7.33 & 3.19 & 2.35 & 3.95 & 0.01810 & 0.11 & -0.61 & 0.94 & 0.01713 & 0.95 & 0.35 & 1.60 & 0.01507 \\
\hline 12.50 & 17.33 & 3.26 & 2.42 & 4.14 & 0.01865 & 0.74 & -0.30 & 1.52 & 0.01759 & 1.41 & 0.66 & 2.17 & 0.01493 \\
\hline 12.92 & 7.33 & 3.21 & 2.44 & 4.03 & 0.01851 & 0.25 & -0.45 & 1.00 & 0.01700 & 0.97 & 0.31 & 1.70 & 0.01425 \\
\hline 12.92 & 17.33 & 3.37 & 2.36 & 4.19 & 0.01941 & 0.78 & -0.09 & 1.56 & 0.02003 & 1.48 & 0.83 & 2.17 & 0.01416 \\
\hline 13.34 & 7.33 & 3.23 & 2.50 & 4.27 & 0.02035 & 0.18 & -0.55 & 0.91 & 0.01876 & 0.95 & 0.46 & 1.55 & 0.01509 \\
\hline 13.34 & 17.33 & 3.31 & 2.48 & 3.97 & 0.01821 & 0.68 & -0.10 & 1.59 & 0.02249 & 1.46 & 0.78 & 2.14 & 0.01676 \\
\hline 13.75 & 7.33 & 3.07 & 2.07 & 4.04 & 0.02121 & 0.18 & -0.55 & 0.99 & 0.01680 & 0.93 & 0.30 & 1.53 & 0.01417 \\
\hline 13.75 & 17.33 & 3.17 & 2.31 & 4.21 & 0.01862 & 0.66 & -0.36 & 1.39 & 0.01661 & 1.36 & 0.69 & 1.99 & 0.01169 \\
\hline 14.22 & 2.67 & 2.97 & 1.87 & 4.16 & 0.00408 & 0.00 & -0.86 & 1.08 & 0.00340 & 1.05 & 0.23 & 1.77 & 0.00279 \\
\hline 14.22 & 3.45 & 2.71 & 1.98 & 3.80 & 0.01528 & 0.20 & -0.77 & 1.19 & 0.01452 & 0.75 & 0.23 & 1.62 & 0.01097 \\
\hline 14.22 & 4.22 & 2.40 & 1.50 & 3.51 & 0.01554 & 0.03 & -0.98 & 0.90 & 0.01413 & 0.75 & 0.34 & 1.54 & 0.00994 \\
\hline 14.22 & 5.00 & 2.76 & 1.87 & 3.96 & 0.01570 & -0.16 & -0.97 & 0.78 & 0.01402 & 0.83 & 0.20 & 1.39 & 0.00939 \\
\hline 14.22 & 5.78 & 3.06 & 1.98 & 4.19 & 0.01655 & 0.03 & -0.88 & 0.78 & 0.01472 & 0.90 & 0.26 & 1.51 & 0.00954 \\
\hline 14.22 & 6.55 & 3.05 & 1.92 & 4.07 & 0.01502 & 0.13 & -0.83 & 0.89 & 0.01292 & 1.00 & 0.29 & 1.67 & 0.00994 \\
\hline 14.22 & 7.33 & 3.04 & 1.90 & 4.09 & 0.00383 & 0.18 & -0.78 & 1.37 & 0.00338 & 1.01 & 0.25 & 1.70 & 0.00262 \\
\hline 14.22 & 12.67 & 3.02 & 1.86 & 4.04 & 0.00330 & 0.35 & -0.83 & 1.22 & 0.00320 & 0.98 & 0.19 & 1.71 & 0.00234 \\
\hline 14.22 & 13.45 & 2.65 & 1.83 & 3.45 & 0.01264 & 0.52 & -0.24 & 1.48 & 0.01245 & 1.10 & 0.58 & 1.68 & 0.00836 \\
\hline 14.22 & 14.22 & 2.73 & 1.79 & 3.75 & 0.01449 & 0.28 & -0.60 & 1.15 & 0.01320 & 1.10 & 0.62 & 1.77 & 0.00854 \\
\hline
\end{tabular}




\begin{tabular}{|c|c|c|c|c|c|c|c|c|c|c|c|c|c|}
\hline 14.22 & 15.00 & 2.94 & 2.05 & 3.93 & 0.01525 & 0.37 & -0.51 & 1.43 & 0.01349 & 1.13 & 0.45 & 1.91 & 0.01184 \\
\hline 14.22 & 15.78 & 3.06 & 2.18 & 4.06 & 0.01415 & 0.57 & -0.34 & 1.49 & 0.01419 & 1.27 & 0.51 & 2.08 & 0.01166 \\
\hline 14.22 & 16.55 & 2.96 & 1.89 & 3.84 & 0.01480 & 0.56 & -0.43 & 1.51 & 0.01527 & 1.35 & 0.52 & 2.10 & 0.01067 \\
\hline 14.22 & 17.33 & 2.97 & 1.94 & 4.08 & 0.00366 & 0.63 & -0.59 & 1.55 & 0.00364 & 1.42 & 0.41 & 2.19 & 0.00262 \\
\hline 14.68 & 2.67 & 3.27 & 2.03 & 4.27 & 0.01549 & -0.01 & -1.02 & 0.89 & 0.01206 & 1.47 & 0.88 & 2.10 & 0.00965 \\
\hline 14.68 & 12.67 & 3.35 & 2.32 & 4.35 & 0.01254 & 0.29 & -0.41 & 1.13 & 0.01121 & 1.53 & 0.82 & 2.16 & 0.00905 \\
\hline 15.15 & 2.67 & 3.33 & 2.50 & 4.34 & 0.01357 & -0.02 & -0.96 & 0.89 & 0.01238 & 1.51 & 0.90 & 2.15 & 0.00937 \\
\hline 15.15 & 12.67 & 3.35 & 2.54 & 4.26 & 0.01265 & 0.24 & -0.63 & 1.03 & 0.01161 & 1.50 & 0.96 & 2.09 & 0.00788 \\
\hline 15.61 & 2.67 & 3.36 & 2.41 & 4.29 & 0.01513 & -0.03 & -1.04 & 0.82 & 0.01362 & 1.50 & 0.93 & 2.07 & 0.00942 \\
\hline 15.61 & 12.67 & 3.38 & 2.48 & 4.31 & 0.01295 & 0.29 & -0.47 & 1.21 & 0.01257 & 1.52 & 1.06 & 2.17 & 0.00892 \\
\hline 16.07 & 2.67 & 3.35 & 2.35 & 4.32 & 0.01348 & -0.02 & -0.90 & 0.88 & 0.01236 & 1.45 & 0.87 & 2.13 & 0.01015 \\
\hline 16.07 & 12.67 & 3.45 & 2.70 & 4.33 & 0.01133 & 0.24 & -0.47 & 0.96 & 0.01024 & 1.46 & 0.94 & 2.16 & 0.00780 \\
\hline 16.54 & 2.67 & 3.36 & 2.41 & 4.34 & 0.01436 & 0.00 & -0.76 & 0.89 & 0.01163 & 1.44 & 0.86 & 2.02 & 0.00859 \\
\hline 16.54 & 12.67 & 3.34 & 2.44 & 4.11 & 0.01200 & 0.28 & -0.47 & 1.04 & 0.01102 & 1.43 & 0.96 & 2.00 & 0.00780 \\
\hline 16.99 & 2.67 & 3.19 & 2.12 & 4.28 & 0.00375 & 0.00 & -0.96 & 0.93 & 0.00315 & 1.35 & 0.71 & 2.02 & 0.00247 \\
\hline 16.99 & 3.45 & 3.21 & 2.29 & 4.51 & 0.01110 & 0.03 & -0.70 & 1.04 & 0.00970 & 1.39 & 0.65 & 2.00 & 0.00739 \\
\hline 16.99 & 4.22 & 3.18 & 2.25 & 4.15 & 0.01029 & 0.18 & -0.68 & 0.92 & 0.00928 & 1.26 & 0.73 & 2.02 & 0.00674 \\
\hline 16.99 & 5.00 & 2.88 & 2.03 & 3.96 & 0.01154 & 0.21 & -0.69 & 1.14 & 0.01043 & 1.25 & 0.70 & 2.09 & 0.00662 \\
\hline 16.99 & 5.78 & 3.09 & 2.02 & 4.20 & 0.01257 & 0.02 & -0.92 & 1.01 & 0.00952 & 1.36 & 0.72 & 2.19 & 0.00685 \\
\hline 16.99 & 6.55 & 3.26 & 2.29 & 4.32 & 0.01151 & 0.17 & -0.69 & 1.10 & 0.00969 & 1.40 & 0.89 & 2.04 & 0.00653 \\
\hline 16.99 & 7.33 & 3.27 & 2.35 & 4.31 & 0.00358 & 0.20 & -0.57 & 1.29 & 0.00294 & 1.47 & 0.85 & 2.24 & 0.00233 \\
\hline 16.99 & 12.67 & 3.20 & 2.33 & 4.15 & 0.00301 & 0.25 & -0.98 & 1.21 & 0.00285 & 1.39 & 0.82 & 2.08 & 0.00211 \\
\hline 16.99 & 13.45 & 3.29 & 2.52 & 4.40 & 0.00919 & 0.33 & -0.68 & 1.53 & 0.0 & 1.43 & 0.94 & 2.02 & 0.00609 \\
\hline 16.99 & 14.22 & 3.13 & 2.06 & 4.23 & 0.01101 & 0.57 & -0.28 & 1.50 & 0.00941 & 1.48 & 0.94 & 1.99 & 0.00636 \\
\hline 16.99 & 15.00 & 2.90 & 2.03 & 3.84 & 0.01019 & 0.32 & -0.58 & 1.26 & 0.00981 & 1.52 & 1.00 & 2.28 & 0.00687 \\
\hline 16.99 & 15.78 & 3.11 & 2.23 & 4.09 & 0.00945 & 0.31 & -0.71 & 1.19 & 0.00919 & 1.52 & 0.97 & 2.26 & 0.00716 \\
\hline 16.99 & 16.55 & 3.08 & 2.13 & 4.11 & 0.01085 & 0.45 & -0.42 & 1.46 & 0.01047 & 1.62 & 1.00 & 2.39 & 0.00855 \\
\hline 16.99 & 17.33 & 3.06 & 2.08 & 4.17 & 0.00357 & 0.46 & -0.50 & 1.46 & 0.00363 & 1.73 & 0.93 & 2.41 & 0.00287 \\
\hline 17.41 & 7.33 & 3.25 & 2.30 & 4.12 & 0.02312 & 0.19 & -0.58 & 1.30 & 935 & 1.42 & 0.91 & 1.94 & 0.01169 \\
\hline 17.41 & 17.33 & 3.08 & 2.37 & 4.02 & 0.02065 & 0.49 & -0.20 & 1.13 & 0.01972 & 1.73 & 0.94 & 2.34 & 0.01892 \\
\hline 17.83 & 7.33 & 3.30 & 2.62 & 4.12 & 0.01888 & 0.18 & -0.45 & 0.85 & 0.01780 & 1.62 & 1.18 & 2.11 & 0.01240 \\
\hline 17.83 & 17.33 & 3.03 & 2.22 & 4.07 & 0.01958 & 0.47 & -0.39 & 1.22 & 0.01855 & 1.83 & 1.37 & 2.47 & 0.01489 \\
\hline 18.24 & 7.33 & 3.31 & 2.41 & 4.16 & 0.02243 & 0.17 & -0.59 & 0.87 & 0.01830 & 1.57 & 1.11 & 2.02 & 0.01326 \\
\hline 18.24 & 17.33 & 3.24 & 2.38 & 4.13 & 0.02108 & 0.51 & -0.24 & 1.23 & 0.02008 & 1.87 & 1.19 & 2.47 & 860 \\
\hline 18.66 & 7.33 & 3.72 & 3.05 & 4.39 & 0.01798 & 0.23 & -0.53 & 1.12 & 0.02042 & 1.68 & 1.02 & 2.17 & 0.01401 \\
\hline 18.66 & 17.33 & 3.54 & 2.66 & 4.28 & 0.02082 & 0.59 & -0.21 & 1.29 & 0.02076 & 1.93 & 1.19 & 2.55 & 0.01700 \\
\hline 19.08 & 7.33 & 3.12 & 2.36 & 4.12 & 0.02477 & 0.15 & -0.53 & 0.68 & 0.01758 & 1.50 & 1.06 & 1.93 & 0.01336 \\
\hline 19.08 & 17.33 & 3.14 & 2.23 & 4.10 & 0.02661 & 0.55 & -0.34 & 1.27 & 0.01989 & 1.71 & 1.14 & 2.25 & 0.01604 \\
\hline 19.47 & 2.67 & 2.95 & 1.97 & 4.13 & 0.00384 & 0.04 & -0.77 & 1.09 & 0.00318 & 1.43 & 0.74 & 2.14 & 0.00252 \\
\hline 19.47 & 3.45 & 2.96 & 1.93 & 3.98 & 0.01111 & 0.04 & -0.76 & 1.03 & 0.00949 & 1.39 & 0.87 & 1.97 & 0.00698 \\
\hline 19.47 & 4.22 & 2.98 & 2.10 & 3.97 & 0.01013 & 0.16 & -0.81 & 1.03 & 0.00847 & 1.27 & 0.70 & 1.96 & 0.00631 \\
\hline 19.47 & 5.00 & 2.73 & 1.82 & 3.83 & 0.01186 & 0.23 & -0.85 & 1.21 & 0.00967 & 1.35 & 0.71 & 1.97 & 0.00654 \\
\hline 19.47 & 5.78 & 2.88 & 1.85 & 4.03 & 0.01209 & -0.10 & -0.82 & 0.62 & 0.00872 & 1.43 & 0.73 & 2.10 & 0.00684 \\
\hline 19.47 & 6.55 & 3.08 & 2.02 & 3.97 & 0.01001 & 0.09 & -0.84 & 0.80 & 0.00884 & 1.46 & 0.67 & 2.00 & 0.00676 \\
\hline 19.47 & 7.33 & 3.07 & 1.94 & 4.17 & 0.00345 & 0.15 & -0.73 & 1.07 & 0.00295 & 1.49 & 0.84 & 2.12 & 0.00228 \\
\hline 19.47 & 12.67 & 2.98 & 2.08 & 4.14 & 0.00303 & 0.19 & -0.66 & 1.10 & 0.00282 & 1.37 & 0.73 & 2.05 & 0.00191 \\
\hline 19.47 & 13.45 & 3.00 & 2.16 & 4.02 & 0.01009 & 0.25 & -0.50 & 1.04 & 0.00867 & 1.36 & 0.86 & 1.89 & 0.00520 \\
\hline 19.47 & 14.22 & 2.98 & 1.91 & 4.10 & 0.01012 & 0.49 & -0.43 & 1.36 & 0.00897 & 1.43 & 0.95 & 2.21 & 0.00565 \\
\hline 19.47 & 15.00 & 2.72 & 1.94 & 3.59 & 0.00929 & 0.28 & -0.70 & 1.10 & 0.00969 & 1.49 & 0.97 & 2.29 & 0.00584 \\
\hline 19.47 & 15.78 & 3.00 & 2.10 & 4.00 & 0.00939 & 0.27 & -0.54 & 1.20 & 0.00902 & 1.47 & 0.97 & 2.10 & 0.00589 \\
\hline 19.47 & 16.55 & 3.00 & 2.25 & 3.85 & 0.00923 & 0.44 & -0.42 & 1.43 & 0.00907 & 1.57 & 0.89 & 2.34 & 0.00772 \\
\hline 19.47 & 17.33 & 3.00 & 2.01 & 4.00 & 0.00364 & 0.48 & -0.48 & 1.46 & 0.00352 & 1.72 & 1.08 & 2.40 & 0.00266 \\
\hline 19.95 & 2.67 & 2.88 & 1.71 & 4.03 & 0.01383 & -0.03 & -0.98 & 0.89 & 0.00999 & 1.89 & 0.77 & 2.82 & 0.01151 \\
\hline 19.95 & 12.67 & 3.00 & 1.70 & 4.08 & 0.01237 & 0.21 & -0.72 & 1.09 & 0.00857 & 1.79 & 0.68 & 2.50 & 0.01006 \\
\hline 20.42 & 2.67 & 3.19 & 2.29 & 4.05 & 0.00939 & -0.04 & -1.02 & 0.99 & 0.00991 & 2.18 & 1.55 & 2.73 & 0.00677 \\
\hline 20.42 & 12.67 & 3.26 & 2.36 & 4.11 & 0.00876 & 0.27 & -0.61 & 1.09 & 0.00904 & 2.08 & 1.55 & 2.68 & 0.00564 \\
\hline 20.90 & 2.67 & 3.17 & 2.31 & 4.01 & 0.00979 & -0.03 & -0.97 & 0.86 & 0.01029 & 2.20 & 1.58 & 2.91 & 0.00692 \\
\hline 20.90 & 12.67 & 3.28 & 2.51 & 4.13 & 0.00873 & 0.23 & -0.60 & 1.08 & 0.00930 & 2.07 & 1.50 & 2.63 & 0.00571 \\
\hline 21.38 & 2.67 & 3.14 & 2.29 & 4.22 & 0.01034 & 0.00 & -0.80 & 0.86 & 0.00969 & 2.15 & 1.61 & 2.87 & 0.00675 \\
\hline
\end{tabular}




\begin{tabular}{|c|c|c|c|c|c|c|c|c|c|c|c|c|c|}
\hline 21.38 & 12.67 & 3.28 & 2.46 & 4.21 & 0.00866 & 0.27 & -0.65 & 1.01 & 0.00922 & 2.08 & 1.51 & 2.69 & 0.00534 \\
\hline 21.85 & 2.67 & 3.29 & 2.27 & 4.31 & 0.01071 & -0.05 & -0.86 & 0.84 & 0.00958 & 2.03 & 1.30 & 2.62 & 0.00769 \\
\hline 21.85 & 12.67 & 3.36 & 2.53 & 4.29 & 0.00978 & 0.24 & -0.64 & 1.07 & 0.00977 & 1.97 & 1.17 & 2.52 & 0.00604 \\
\hline 22.37 & 2.67 & 3.14 & 2.23 & 4.13 & 0.00342 & -0.02 & -0.86 & 0.99 & 0.00344 & 1.91 & 1.27 & 2.53 & 0.00243 \\
\hline 22.37 & 3.45 & 3.18 & 2.11 & 4.06 & 0.00963 & 0.04 & -0.76 & 0.96 & 0.01023 & 1.88 & 1.22 & 2.45 & 0.00758 \\
\hline 22.37 & 4.22 & 3.24 & 2.42 & 4.12 & 0.00966 & 0.24 & -0.78 & 1.12 & 0.01024 & 1.82 & 1.23 & 2.30 & 0.00618 \\
\hline 22.37 & 5.00 & 2.92 & 2.03 & 4.10 & 0.01089 & 0.22 & -0.82 & 1.28 & 0.01175 & 1.89 & 1.18 & 2.52 & 0.00626 \\
\hline 22.37 & 5.78 & 3.17 & 2.36 & 4.14 & 0.00999 & -0.02 & -0.81 & 0.92 & 0.01039 & 1.89 & 1.28 & 2.37 & 0.00698 \\
\hline 22.37 & 6.55 & 3.26 & 2.39 & 4.07 & 0.00989 & 0.18 & -0.84 & 1.01 & 0.00976 & 1.94 & 1.16 & 2.63 & 0.00761 \\
\hline 22.37 & 7.33 & 3.23 & 2.31 & 4.09 & 0.00325 & 0.13 & -0.71 & 1.29 & 0.00311 & 1.95 & 1.30 & 2.64 & 0.00243 \\
\hline 22.37 & 12.67 & 3.21 & 2.38 & 4.10 & 0.00291 & 0.22 & -0.78 & 1.09 & 0.00305 & 1.92 & 1.25 & 2.44 & 0.00193 \\
\hline 22.37 & 13.45 & 3.26 & 2.51 & 4.01 & 0.00791 & 0.31 & -0.63 & 1.12 & 0.00931 & 1.93 & 1.48 & 2.63 & 0.00538 \\
\hline 22.37 & 14.22 & 3.05 & 2.02 & 3.92 & 0.00872 & 0.54 & -0.26 & 1.36 & 0.00859 & 1.97 & 1.15 & 2.41 & 0.00531 \\
\hline 22.37 & 15.00 & 2.86 & 2.07 & 4.02 & 0.00916 & 0.19 & -0.55 & 1.06 & 0.00879 & 2.01 & 1.52 & 2.53 & 0.00551 \\
\hline 22.37 & 15.78 & 3.07 & 2.21 & 3.91 & 0.00811 & 0.37 & -0.66 & 1.33 & 0.00928 & 2.00 & 1.48 & 2.78 & 0.00622 \\
\hline 22.37 & 16.55 & 2.96 & 2.19 & 3.96 & 0.00865 & 0.52 & -0.47 & 1.49 & 0.00945 & 2.12 & 1.43 & 2.84 & 0.00748 \\
\hline 22.37 & 17.33 & 2.92 & 1.99 & 3.92 & 0.00291 & 0.49 & -0.42 & 1.40 & 0.00303 & 2.17 & 1.47 & 2.97 & 0.00244 \\
\hline 22.78 & 7.33 & 3.17 & 2.40 & 4.03 & 0.00716 & 0.10 & -0.81 & 1.05 & 0.00738 & 2.15 & 1.40 & 2.80 & 0.00533 \\
\hline 22.78 & 17.33 & 2.87 & 2.10 & 3.89 & 0.00691 & 0.43 & -0.50 & 1.37 & 0.00719 & 2.34 & 1.56 & 3.15 & 0.00573 \\
\hline 23.19 & 7.33 & 3.16 & 2.33 & 4.16 & 0.00752 & 0.11 & -0.76 & 1.18 & 0.00758 & 2.13 & 1.49 & 2.73 & 0.00519 \\
\hline 23.19 & 17.33 & 2.88 & 1.98 & 3.90 & 0.00678 & 0.42 & -0.43 & 1.40 & 0.00698 & 2.30 & 1.66 & 3.16 & 0.00533 \\
\hline 23.60 & 7.33 & 3.17 & 2.28 & 4.04 & 0.00753 & 0.11 & -0.68 & 1.02 & 0.00726 & 2.11 & 1.51 & 2.83 & 0.00518 \\
\hline 23.60 & 17.33 & 2.84 & 1.84 & 3.65 & 0.00646 & 0.41 & -0.41 & 1.34 & 0.00735 & 2.30 & 1.65 & 2.99 & 0.00525 \\
\hline 24.01 & 7.33 & 3.14 & 2.33 & 4.18 & 0.00712 & 0.12 & -0.76 & 1.04 & 0.00755 & 2.13 & 1.58 & 2.78 & 0.00532 \\
\hline 24.01 & 17.33 & 2.88 & 2.03 & 3.73 & 0.00711 & 0.41 & -0.37 & 1.28 & 0.00734 & 2.27 & 1.71 & 3.21 & 0.00544 \\
\hline 24.42 & 7.33 & 3.06 & 1.92 & 4.19 & 0.00895 & 0.11 & -0.81 & 1.14 & 0.00760 & 2.05 & 1.33 & 2.61 & 0.00587 \\
\hline 24.42 & 17.33 & 2.87 & 1.93 & 4.03 & 0.00863 & 0.33 & -0.64 & 1.36 & 0.00780 & 2.23 & 1.57 & 2.95 & 0.00573 \\
\hline 24.97 & 2.67 & 2.67 & 1.69 & 3.75 & 0.00306 & 0.01 & -0.87 & 0.86 & 0.00314 & 2.19 & 1.49 & 2.86 & 0.00231 \\
\hline 24.97 & 3.45 & 2.76 & 1.78 & 3.59 & 0.00995 & 0.02 & -0.83 & 1.01 & 0.00976 & 2.13 & 1.54 & 2.65 & 0.00660 \\
\hline 24.97 & 4.22 & 2.85 & 2.09 & 4.11 & 0.00923 & 0.15 & -0.86 & 0.93 & 0.00993 & 2.03 & 1.46 & 2.74 & 0.00689 \\
\hline 24.97 & 5.00 & 2.67 & 1.78 & 3.68 & 0.01064 & 0.26 & -0.75 & 1.22 & 0.01098 & 2.08 & 1.56 & 2.83 & 0.00719 \\
\hline 24.97 & 5.78 & 2.84 & 1.69 & 3.95 & 0.01174 & -0.10 & -0.86 & 0.93 & 0.01011 & 2.06 & 1.32 & 2.70 & 0.00731 \\
\hline 24.97 & 6.55 & 2.97 & 1.97 & 3.72 & 0.00949 & 0.08 & -0.83 & 1.22 & 0.00979 & 2.09 & 1.37 & 2.65 & 0.00762 \\
\hline 24.97 & 7.33 & 2.90 & 1.95 & 3.92 & 0.00333 & 0.12 & -0.67 & 1.25 & 0.00338 & 2.08 & 1.39 & 2.74 & 0.00254 \\
\hline 24.97 & 12.67 & 2.96 & 2.18 & 3.90 & 0.00275 & 0.17 & -0.79 & 1.00 & 0.00300 & 2.12 & 1.42 & 2.78 & 0.00196 \\
\hline 24.97 & 13.45 & 3.00 & 2.07 & 4.00 & 0.00867 & 0.21 & -0.76 & 1.09 & 0.00982 & 2.14 & 1.48 & 2.68 & 0.00620 \\
\hline 24.97 & 14.22 & 2.92 & 2.21 & 3.73 & 0.00771 & 0.44 & -0.50 & 1.27 & 0.00850 & 2.21 & 1.55 & 2.80 & 0.00534 \\
\hline 24.97 & 15.00 & 2.72 & 1.96 & 3.69 & 0.00880 & 0.17 & -0.59 & 0.99 & 0.00879 & 2.17 & 1.35 & 2.73 & 0.00641 \\
\hline 24.97 & 15.78 & 2.99 & 2.17 & 3.81 & 0.00848 & 0.08 & -0.83 & 0.84 & 0.00844 & 2.13 & 1.40 & 2.63 & 0.00622 \\
\hline 24.97 & 16.55 & 2.82 & 2.04 & 3.82 & 0.00879 & 0.26 & -0.54 & 1.27 & 0.00855 & 2.15 & 1.45 & 2.82 & 0.00627 \\
\hline 24.97 & 17.33 & 2.72 & 1.71 & 3.70 & 0.00325 & 0.28 & -0.59 & 1.27 & 0.00337 & 2.21 & 1.57 & 2.90 & 0.00229 \\
\hline 25.42 & 2.67 & 2.69 & 1.89 & 3.29 & 0.02010 & -0.02 & -0.66 & 0.90 & 0.02039 & 2.17 & 1.45 & 2.71 & 0.01520 \\
\hline 25.42 & 12.67 & 3.01 & 2.38 & 3.71 & 0.01771 & 0.16 & -0.54 & 0.80 & 0.01830 & 2.04 & 1.33 & 2.66 & 0.01363 \\
\hline 25.87 & 2.67 & 2.71 & 2.14 & 3.37 & 0.02554 & -0.01 & -0.72 & 0.58 & 0.02747 & 2.20 & 1.82 & 2.64 & 0.01794 \\
\hline 25.87 & 12.67 & 2.96 & 2.36 & 3.60 & 0.02459 & 0.13 & -0.47 & 0.74 & 0.02525 & 2.12 & 1.73 & 2.46 & 0.01627 \\
\hline 26.32 & 2.67 & 2.44 & 1.88 & 3.46 & 0.02286 & 0.03 & -0.69 & 0.84 & 0.02312 & 2.31 & 1.53 & 3.19 & 0.02969 \\
\hline 26.32 & 12.67 & 2.73 & 2.06 & 3.63 & 0.02500 & 0.24 & -0.52 & 0.96 & 0.02305 & 2.20 & 1.38 & 3.15 & 0.03182 \\
\hline 26.77 & 2.67 & 3.00 & 2.19 & 3.73 & 0.02924 & -0.01 & -0.61 & 0.62 & 0.02096 & 2.76 & 2.21 & 3.17 & 0.01850 \\
\hline 26.77 & 12.67 & 3.16 & 2.42 & 3.86 & 0.02362 & 0.20 & -0.55 & 0.94 & 0.02112 & 2.77 & 2.13 & 3.27 & 0.01836 \\
\hline 27.22 & 2.67 & 2.85 & 2.32 & 3.58 & 0.02549 & 0.04 & -0.54 & 0.70 & 0.02707 & 2.81 & 2.35 & 3.17 & 0.01533 \\
\hline 27.22 & 12.67 & 3.06 & 2.17 & 3.62 & 0.02671 & 0.20 & -0.46 & 0.80 & 0.02947 & 2.81 & 2.45 & 3.20 & 0.01574 \\
\hline 27.67 & 2.67 & 2.82 & 1.74 & 4.02 & 0.00344 & 0.09 & -0.71 & 1.05 & 0.00338 & 2.83 & 2.22 & 3.40 & 0.00223 \\
\hline 27.67 & 3.45 & 2.96 & 1.54 & 4.21 & 0.01125 & 0.13 & -1.22 & 1.42 & 0.01155 & 2.76 & 2.11 & 3.37 & 0.00622 \\
\hline 27.67 & 4.22 & 2.96 & 1.56 & 4.18 & 0.01115 & 0.31 & -1.10 & 1.39 & 0.01166 & 2.67 & 2.20 & 3.20 & 0.00577 \\
\hline 27.67 & 5.00 & 2.60 & 1.54 & 3.90 & 0.01137 & 0.19 & -1.08 & 1.31 & 0.01258 & 2.68 & 2.02 & 3.38 & 0.00623 \\
\hline 27.67 & 5.78 & 2.85 & 1.58 & 3.99 & 0.01115 & -0.05 & -1.09 & 1.49 & 0.01121 & 2.75 & 2.21 & 3.35 & 0.00648 \\
\hline 27.67 & 6.55 & 2.90 & 1.87 & 4.04 & 0.01068 & 0.11 & -1.17 & 1.31 & 0.01131 & 2.78 & 1.96 & 3.32 & 0.00654 \\
\hline 27.67 & 7.33 & 2.88 & 1.91 & 3.75 & 0.00345 & 0.18 & -0.84 & 1.26 & 0.00363 & 2.78 & 2.06 & 3.38 & 0.00238 \\
\hline 27.67 & 12.67 & 2.99 & 2.11 & 3.99 & 0.00307 & 0.22 & -0.71 & 1.11 & 0.00320 & 2.84 & 2.11 & 3.35 & 0.00218 \\
\hline
\end{tabular}




\begin{tabular}{|c|c|c|c|c|c|c|c|c|c|c|c|c|c|}
\hline 27.67 & 13.45 & 2.99 & 2.11 & 3.90 & 0.00907 & 0.34 & -0.63 & 1.48 & 0.01033 & 2.87 & 2.19 & 3.46 & 0.00644 \\
\hline 27.67 & 14.22 & 2.78 & 2.01 & 3.89 & 0.01044 & 0.46 & -0.67 & 1.41 & 0.01051 & 2.86 & 2.25 & 3.41 & 0.00582 \\
\hline 27.67 & 15.00 & 2.82 & 2.00 & 3.85 & 0.01081 & 0.08 & -0.73 & 1.25 & 0.01007 & 2.82 & 2.23 & 3.36 & 0.00621 \\
\hline 27.67 & 15.78 & 3.18 & 2.23 & 3.96 & 0.00992 & 0.18 & -0.75 & 1.46 & 0.01089 & 2.81 & 2.27 & 3.41 & 0.00578 \\
\hline 27.67 & 16.55 & 3.06 & 2.05 & 3.99 & 0.01095 & 0.24 & -0.71 & 1.43 & 0.01075 & 2.87 & 2.34 & 3.48 & 0.00619 \\
\hline 27.67 & 17.33 & 2.90 & 1.92 & 4.06 & 0.00356 & 0.16 & -0.87 & 1.28 & 0.00333 & 2.90 & 2.23 & 3.58 & 0.00207 \\
\hline 28.07 & 7.33 & 2.83 & 2.15 & 3.36 & 0.03099 & 0.22 & -0.40 & 0.89 & 0.03364 & 2.68 & 2.22 & 3.19 & 0.02429 \\
\hline 28.07 & 17.33 & 2.78 & 1.92 & 3.65 & 0.04021 & 0.09 & -0.69 & 0.84 & 0.03083 & 2.82 & 2.43 & 3.21 & 0.02113 \\
\hline 28.47 & 7.33 & 2.92 & 2.07 & 3.49 & 0.03159 & 0.10 & -0.52 & 0.71 & 0.03018 & 2.87 & 2.27 & 3.29 & 0.02309 \\
\hline 28.47 & 17.33 & 2.89 & 2.26 & 3.74 & 0.03143 & 0.14 & -0.43 & 0.90 & 0.02809 & 3.04 & 2.34 & 3.53 & 0.02631 \\
\hline 28.88 & 7.33 & 2.81 & 1.97 & 3.35 & 0.03233 & 0.19 & -0.53 & 0.83 & 0.03454 & 3.12 & 2.71 & 3.73 & 0.02463 \\
\hline 28.88 & 17.33 & 3.17 & 2.35 & 3.85 & 0.03371 & 0.37 & -0.40 & 1.07 & 0.03086 & 3.26 & 2.87 & 4.01 & 0.02536 \\
\hline 29.28 & 7.33 & 2.57 & 1.91 & 3.36 & 0.03376 & 0.23 & -0.54 & 0.88 & 0.02977 & 3.15 & 2.79 & 3.58 & 0.01805 \\
\hline 29.28 & 17.33 & 2.73 & 1.98 & 3.26 & 0.03173 & 0.14 & -0.70 & 1.00 & 0.03442 & 3.52 & 3.15 & 3.96 & 0.02199 \\
\hline 29.68 & 7.33 & 2.56 & 1.95 & 3.41 & 0.03856 & 0.28 & -0.32 & 0.77 & 0.02652 & 3.13 & 2.73 & 3.41 & 0.01535 \\
\hline 29.68 & 17.33 & 2.79 & 1.88 & 3.70 & 0.03925 & 0.23 & -0.87 & 1.02 & 0.03470 & 3.52 & 3.04 & 3.88 & 0.01771 \\
\hline 30.10 & 2.67 & 2.55 & 2.00 & 3.41 & 0.00642 & 0.17 & -0.47 & 0.75 & 0.00581 & 3.24 & 2.79 & 3.94 & 0.00425 \\
\hline 30.10 & 3.45 & 2.56 & 1.77 & 3.23 & 0.00927 & 0.18 & -0.48 & 0.87 & 0.00835 & 3.22 & 2.80 & 3.69 & 0.00609 \\
\hline 30.10 & 4.22 & 2.64 & 1.86 & 3.19 & 0.00908 & 0.27 & -0.37 & 0.89 & 0.00898 & 3.10 & 2.64 & 3.61 & 0.00577 \\
\hline 30.10 & 5.00 & 2.41 & 1.78 & 3.09 & 0.01029 & 0.27 & -0.40 & 0.84 & 0.00891 & 3.11 & 2.70 & 3.63 & 0.00632 \\
\hline 30.10 & 5.78 & 2.53 & 1.88 & 3.22 & 0.01021 & -0.03 & -0.50 & 0.59 & 0.00848 & 3.14 & 2.70 & 3.63 & 0.00615 \\
\hline 30.10 & 6.55 & 2.60 & 1.97 & 3.20 & 0.00919 & 0.16 & -0.45 & 0.79 & 0.00873 & 3.12 & 2.69 & 3.57 & 0.00567 \\
\hline 30.10 & 7.33 & 2.56 & 2.02 & 3.29 & 0.00678 & 0.20 & -0.37 & 0.87 & 0.00628 & 3.12 & 2.60 & 3.67 & 0.00437 \\
\hline 30.10 & 12.67 & 2.64 & 1.89 & 3.43 & 0.00634 & 0.14 & -0.58 & 0.84 & 0.00587 & 3.21 & 2.65 & 3.76 & 0.00439 \\
\hline 30.10 & 13.45 & 2.66 & 2.06 & 3.33 & 0.00902 & 0.16 & -0.40 & 0.87 & 0.00837 & 3.22 & 2.81 & 3.63 & 0.00570 \\
\hline 30.10 & 14.22 & 2.59 & 1.84 & 3.32 & 0.00983 & 0.33 & -0.51 & 0.93 & 0.00929 & 3.29 & 2.70 & 3.99 & 0.00642 \\
\hline 30.10 & 15.00 & 2.35 & 1.66 & 3.04 & 0.00959 & 0.15 & -0.66 & 1.00 & 0.01081 & 3.30 & 2.94 & 3.81 & 0.00558 \\
\hline 30.10 & 15.78 & 2.75 & 1.78 & 3.38 & 0.00906 & 0.14 & -0.65 & 0.77 & 0.00927 & 3.27 & 2.86 & 3.71 & 0.00561 \\
\hline 30.10 & 16.55 & 2.77 & 1.92 & 3.42 & 0.00937 & 0.21 & -0.47 & 1.04 & 0.00921 & 3.39 & 2.90 & 4.06 & 0.00633 \\
\hline 30.10 & 17.33 & 2.74 & 1.86 & 3.52 & 0.00718 & 0.20 & -0.54 & 1.09 & 0.00672 & 3.45 & 2.99 & 4.00 & 0.00478 \\
\hline 30.11 & 2.67 & 2.44 & 1.40 & 3.55 & 0.00736 & 0.06 & -0.55 & 0.74 & 0.00645 & 3.32 & 2.87 & 3.86 & 0.00404 \\
\hline 30.11 & 3.45 & 2.52 & 1.40 & 3.75 & 0.01257 & 0.18 & -0.66 & 0.97 & 0.01093 & 3.29 & 2.86 & 3.81 & 0.00676 \\
\hline 30.11 & 4.22 & 2.61 & 1.38 & 3.35 & 0.01041 & 0.26 & -0.64 & 0.99 & 0.00986 & 3.16 & 2.57 & 3.67 & 0.00583 \\
\hline 30.11 & 5.00 & 2.64 & 1.37 & 3.81 & 0.01199 & 0.26 & -0.56 & 0.98 & 0.01126 & 3.19 & 2.72 & 3.64 & 0.00660 \\
\hline 30.11 & 5.78 & 2.44 & 1.46 & 3.79 & 0.01240 & 0.14 & -0.64 & 0.87 & 0.01218 & 3.17 & 2.53 & 3.65 & 0.00647 \\
\hline 30.11 & 6.55 & 2.63 & 1.47 & 3.86 & 0.01253 & 0.03 & -0.63 & 0.93 & 0.01089 & 3.18 & 2.61 & 3.65 & 0.00628 \\
\hline 30.11 & 7.33 & 2.59 & 2.17 & 3.00 & 0.01911 & 0.22 & -0.05 & 0.50 & 0.01399 & 3.15 & 2.93 & 3.37 & 0.01226 \\
\hline 30.11 & 12.67 & 2.74 & 2.01 & 3.48 & 0.00680 & 0.11 & -0.50 & 0.75 & 0.00605 & 3.18 & 2.57 & 3.79 & 0.00451 \\
\hline 30.11 & 13.45 & 2.77 & 1.79 & 3.72 & 0.01154 & 0.11 & -0.49 & 0.95 & 0.01067 & 3.24 & 2.53 & 3.74 & 0.00690 \\
\hline 30.11 & 14.22 & 2.69 & 1.65 & 3.87 & 0.01089 & 0.28 & -0.46 & 1.00 & 0.01091 & 3.25 & 2.71 & 3.82 & 0.00700 \\
\hline 30.11 & 15.00 & 2.62 & 1.73 & 3.69 & 0.01172 & 0.34 & -0.44 & 0.98 & 0.01081 & 3.31 & 2.67 & 3.86 & 0.00694 \\
\hline 30.11 & 15.78 & 2.57 & 1.61 & 3.84 & 0.01336 & 0.07 & -0.50 & 0.89 & 0.01091 & 3.31 & 2.77 & 3.76 & 0.00632 \\
\hline 30.11 & 16.55 & 2.94 & 1.92 & 3.77 & 0.01187 & 0.14 & -0.49 & 0.99 & 0.01090 & 3.31 & 2.85 & 3.98 & 0.00653 \\
\hline 30.11 & 17.33 & 2.88 & 2.57 & 3.18 & 0.01439 & 0.22 & -0.02 & 0.51 & 0.01221 & 3.42 & 3.25 & 3.62 & 0.01125 \\
\hline 30.20 & 7.33 & 2.16 & 1.92 & 2.41 & 0.07169 & 0.23 & 0.10 & 0.42 & 0.04788 & 3.01 & 2.88 & 3.09 & 0.02925 \\
\hline 30.20 & 17.33 & 2.40 & 2.32 & 2.58 & 0.06157 & 0.13 & 0.04 & 0.27 & 0.05087 & 3.36 & 3.28 & 3.49 & 0.04493 \\
\hline 30.29 & 7.33 & 2.38 & 2.19 & 2.62 & 0.05704 & 0.19 & 0.06 & 0.40 & 0.05331 & 3.14 & 3.04 & 3.26 & 0.03185 \\
\hline 30.29 & 17.33 & 2.56 & 2.46 & 2.66 & 0.02785 & 0.19 & 0.04 & 0.35 & 0.04588 & 3.51 & 3.38 & 3.62 & 0.03632 \\
\hline 30.38 & 7.33 & 2.58 & 2.34 & 2.78 & 0.05438 & 0.23 & 0.07 & 0.32 & 0.03786 & 3.13 & 3.00 & 3.17 & 0.02169 \\
\hline 30.38 & 17.33 & 2.77 & 2.59 & 3.01 & 0.05518 & 0.22 & 0.12 & 0.32 & 0.03161 & 3.38 & 3.26 & 3.52 & 0.03569 \\
\hline 30.46 & 7.33 & 2.49 & 2.39 & 2.63 & 0.03133 & 0.33 & 0.20 & 0.46 & 0.03905 & 3.13 & 3.01 & 3.33 & 0.03831 \\
\hline 30.46 & 17.33 & 2.90 & 2.77 & 3.00 & 0.03181 & 0.18 & 0.04 & 0.40 & 0.05002 & 3.38 & 3.25 & 3.52 & 0.03389 \\
\hline 30.55 & 7.33 & 2.62 & 2.45 & 2.74 & 0.03879 & 0.27 & 0.13 & 0.39 & 0.03554 & 3.16 & 3.01 & 3.31 & 0.03967 \\
\hline 30.55 & 17.33 & 2.86 & 2.60 & 3.03 & 0.06439 & 0.26 & 0.18 & 0.32 & 0.02325 & 3.45 & 3.33 & 3.53 & 0.03455 \\
\hline 30.64 & 2.67 & 2.16 & 1.92 & 2.37 & 0.01032 & 0.09 & -0.19 & 0.32 & 0.00970 & 3.30 & 3.15 & 3.42 & 0.00562 \\
\hline 30.64 & 3.45 & 2.24 & 1.97 & 2.56 & 0.02237 & 0.15 & -0.01 & 0.39 & 0.01480 & 3.20 & 3.05 & 3.33 & 0.01244 \\
\hline 30.64 & 4.22 & 2.29 & 2.09 & 2.51 & 0.01590 & 0.19 & -0.01 & 0.47 & 0.01807 & 3.15 & 2.93 & 3.32 & 0.01243 \\
\hline 30.64 & 5.00 & 2.12 & 1.78 & 2.42 & 0.02676 & 0.22 & -0.20 & 0.47 & 0.02409 & 3.14 & 2.99 & 3.28 & 0.01326 \\
\hline 30.64 & 5.78 & 2.22 & 1.80 & 2.55 & 0.02721 & 0.06 & -0.22 & 0.40 & 0.02494 & 3.14 & 2.95 & 3.34 & 0.01406 \\
\hline
\end{tabular}




\begin{tabular}{|c|c|c|c|c|c|c|c|c|c|c|c|c|c|}
\hline 30.64 & 6.55 & 2.24 & 2.05 & 2.52 & 0.02078 & 0.20 & 0.07 & 0.39 & 0.01424 & 3.11 & 2.90 & 3.40 & 0.01666 \\
\hline 30.64 & 7.33 & 2.21 & 1.90 & 2.52 & 0.01803 & 0.24 & 0.02 & 0.41 & 0.01367 & 3.05 & 2.89 & 3.29 & 0.01326 \\
\hline 30.64 & 12.67 & 2.40 & 2.11 & 2.64 & 0.01017 & 0.02 & -0.18 & 0.24 & 0.01011 & 3.17 & 3.03 & 3.38 & 0.00773 \\
\hline 30.64 & 13.45 & 2.37 & 2.14 & 2.68 & 0.02160 & 0.05 & -0.12 & 0.21 & 0.01544 & 3.16 & 3.00 & 3.33 & 0.01420 \\
\hline 30.64 & 14.22 & 2.36 & 2.05 & 2.59 & 0.02222 & 0.22 & -0.01 & 0.47 & 0.02240 & 3.23 & 3.06 & 3.43 & 0.01409 \\
\hline 30.64 & 15.00 & 2.08 & 1.82 & 2.23 & 0.01804 & 0.13 & -0.23 & 0.49 & 0.02937 & 3.29 & 3.09 & 3.48 & 0.01491 \\
\hline 30.64 & 15.78 & 2.40 & 2.12 & 2.59 & 0.01735 & 0.13 & -0.13 & 0.40 & 0.01996 & 3.27 & 3.11 & 3.51 & 0.01587 \\
\hline 30.64 & 16.55 & 2.41 & 2.14 & 2.63 & 0.02027 & 0.17 & 0.01 & 0.44 & 0.01580 & 3.41 & 3.06 & 3.62 & 0.01853 \\
\hline 30.64 & 17.33 & 2.44 & 2.01 & 2.74 & 0.02026 & 0.14 & 0.00 & 0.41 & 0.01443 & 3.40 & 3.05 & 3.55 & 0.01322 \\
\hline 30.69 & 2.67 & 2.22 & 2.06 & 2.43 & 0.04984 & 0.04 & -0.02 & 0.12 & 0.01820 & 3.29 & 3.16 & 3.41 & 0.03305 \\
\hline 30.69 & 12.67 & 2.36 & 2.18 & 2.53 & 0.04854 & 0.04 & -0.07 & 0.24 & 0.03988 & 3.14 & 3.07 & 3.29 & 0.02850 \\
\hline 30.78 & 2.67 & 2.19 & 1.98 & 2.31 & 0.03919 & 0.13 & -0.06 & 0.28 & 0.04057 & 3.26 & 3.14 & 3.41 & 0.03119 \\
\hline 30.78 & 12.67 & 2.40 & 2.22 & 2.53 & 0.04593 & 0.05 & -0.05 & 0.25 & 0.04240 & 3.10 & 2.97 & 3.25 & 0.03171 \\
\hline 30.86 & 2.67 & 2.12 & 2.02 & 2.20 & 0.03167 & 0.13 & 0.01 & 0.28 & 0.04036 & 3.49 & 3.27 & 3.70 & 0.06425 \\
\hline 30.86 & 12.67 & 2.40 & 2.17 & 2.62 & 0.05609 & 0.02 & -0.12 & 0.18 & 0.04847 & 3.37 & 3.10 & 3.80 & 0.09275 \\
\hline 30.94 & 2.67 & 2.03 & 1.93 & 2.13 & 0.03058 & 0.00 & -0.09 & 0.14 & 0.03533 & 3.99 & 3.92 & 4.04 & 0.01705 \\
\hline 30.94 & 12.67 & 2.35 & 2.22 & 2.48 & 0.03907 & 0.06 & 0.00 & 0.14 & 0.02195 & 3.82 & 3.71 & 3.92 & 0.03205 \\
\hline 31.03 & 2.67 & 2.05 & 1.88 & 2.19 & 0.04149 & 0.12 & 0.05 & 0.21 & 0.02478 & 4.00 & 3.98 & $\begin{array}{ll}4.07 \\
\end{array}$ & 0.01198 \\
\hline 31.03 & 12.67 & 2.46 & 2.20 & 2.75 & 0.07237 & 0.03 & -0.18 & 0.17 & 0.04261 & 3.82 & 3.73 & 4.01 & 0.04171 \\
\hline 31.11 & 2.67 & 2.11 & 1.84 & 2.43 & 0.01423 & 0.00 & -0.38 & 0.35 & 0.01479 & 4.00 & 3.82 & 4.21 & 0.01047 \\
\hline 31.11 & 3.45 & 2.16 & 1.79 & 2.58 & 0.01187 & 0.23 & -0.15 & 0.54 & 0.01154 & 4.00 & 3.79 & 4.19 & 0.00680 \\
\hline 31.11 & 4.22 & 2.28 & 1.98 & 2.60 & 0.01052 & 0.31 & 0.05 & 0.57 & 0.01119 & 3.92 & 3.73 & 4.15 & 0.00839 \\
\hline 31.11 & 5.00 & 2.13 & 1.67 & 2.44 & 0.01499 & 0.14 & -0.32 & 0.57 & 0.0 & 3.81 & 3.59 & 4.07 & 0.00842 \\
\hline 31.11 & 5.78 & 2.31 & 1.89 & 2.67 & 0.01460 & 0.14 & -0.13 & 0.40 & 0.01193 & 3.82 & 3.53 & 4.00 & 0.00758 \\
\hline 31.11 & 6.55 & 2.28 & 1.91 & 2.69 & 0.01476 & 0.17 & -0.10 & 0.54 & 0.01126 & 3.82 & 3.62 & 4.05 & 0.00859 \\
\hline 31.11 & 7.33 & 2.24 & 1.83 & 2.58 & 0.01692 & 0.21 & -0.11 & 0.51 & 0.01201 & 3.85 & 3.68 & 4.17 & 0.00812 \\
\hline 31.11 & 12.67 & 2.36 & 1.99 & 2.70 & 0.01710 & 0.04 & -0.18 & 0.30 & 0.01251 & 3.80 & 3.65 & 4.04 & 0.01057 \\
\hline 31.11 & 13.45 & 2.40 & 2.09 & 2.67 & 0.01301 & 0.08 & -0.18 & 0.35 & 0.01074 & 3.85 & 3.55 & 4.14 & 0.00900 \\
\hline 31.11 & 14.22 & 2.27 & 1.78 & 2.70 & 0.01827 & 0.24 & -0.02 & 0.63 & 0.01277 & 3.94 & 3.71 & 4.17 & 0.00758 \\
\hline 31.11 & 15.00 & 2.23 & 1.82 & 2.73 & 0.01541 & 0.00 & -0.27 & 0.31 & 0.01128 & 3.97 & 3.79 & 4.15 & 0.00748 \\
\hline 31.11 & 15.78 & 2.39 & 2.12 & 2.74 & 0.00867 & 0.13 & -0.20 & 0.37 & 0.01005 & 4.07 & 3.88 & 4.34 & 0.00727 \\
\hline 31.11 & 16.55 & 2.34 & 2.09 & 2.58 & 0.00918 & 0.12 & -0.11 & 0.41 & 0.00994 & 4.07 & 3.83 & 4.41 & 0.00841 \\
\hline 31.11 & 17.33 & 2.33 & 2.10 & 2.55 & 0.01041 & 0.09 & -0.19 & 0.50 & 0.01105 & 4.14 & 3.98 & 4.32 & 0.00785 \\
\hline 31.19 & 7.33 & 2.19 & 1.89 & 2.34 & 0.06531 & 0.27 & 0.08 & 0.67 & 0.09275 & 3.81 & 3.66 & 3.99 & 0.05444 \\
\hline 31.19 & 17.33 & 2.40 & 2.33 & 2.54 & 0.03198 & 0.02 & -0.06 & 0.15 & 0.03154 & 4.06 & 4.02 & 4.12 & 0.01823 \\
\hline 31.28 & 7.33 & 2.25 & 2.09 & 2.40 & 0.04138 & 0.21 & 0.12 & 0.31 & 0.02828 & 3.78 & 3.69 & 3.83 & 0.01940 \\
\hline 31.28 & 17.33 & 2.36 & 2.17 & 2.50 & 0.04426 & 0.13 & 0.04 & 0.23 & 0.02547 & 4.14 & 4.02 & 4.28 & 0.03774 \\
\hline 31.36 & 7.33 & 2.16 & 1.88 & 2.40 & 0.09115 & 0.13 & 0.02 & 0.22 & 0.03460 & 3.58 & 3.44 & 3.73 & 0.06209 \\
\hline 31.36 & 17.33 & 2.32 & 2.16 & 2.49 & 0.05283 & 0.15 & 0.00 & 0.22 & 0.03921 & 3.91 & 3.67 & 4.07 & 0.06839 \\
\hline 31.44 & 7.33 & 2.31 & 2.06 & 2.56 & 0.06982 & 0.21 & 0.10 & 0.28 & 0.03556 & 3.45 & 3.26 & 3.60 & 0.05794 \\
\hline 31.44 & 17.33 & 2.27 & 2.22 & 2.35 & 0.02072 & 0.10 & 0.00 & 0.25 & 0.04023 & 3.76 & 3.57 & 3.93 & 0.05092 \\
\hline 31.53 & 7.33 & 2.06 & 1.77 & 2.28 & 0.09539 & 0.15 & -0.09 & 0.26 & 0.06498 & 3.70 & 3.50 & 3.90 & 0.06758 \\
\hline 31.53 & 17.33 & 2.26 & 2.07 & 2.41 & 0.06314 & 0.15 & -0.04 & 0.29 & 0.05978 & 4.11 & 3.86 & 4.29 & 0.07680 \\
\hline 31.61 & 2.67 & 2.35 & 2.14 & 2.63 & 0.01133 & -0.04 & -0.32 & 0.23 & 0.01237 & 4.39 & 4.19 & 4.61 & 0.00872 \\
\hline 31.61 & 3.45 & 2.43 & 2.15 & 2.71 & 0.01610 & 0.18 & -0.11 & 0.43 & 0.01445 & 4.34 & 4.17 & 4.48 & 0.00917 \\
\hline 31.61 & 4.22 & 2.49 & 2.29 & 2.74 & 0.01352 & 0.27 & -0.02 & 0.52 & 0.01324 & 4.24 & 4.11 & 4.44 & 0.00925 \\
\hline 31.61 & 5.00 & 2.26 & 1.92 & 2.65 & 0.01897 & 0.16 & -0.20 & 0.59 & 0.02544 & 4.13 & 3.93 & 4.36 & 0.01141 \\
\hline 31.61 & 5.78 & 2.54 & 2.08 & 2.90 & 0.02079 & -0.02 & -0.35 & 0.29 & 0.01887 & 4.17 & 4.00 & 4.47 & 0.01174 \\
\hline 31.61 & 6.55 & 2.49 & 2.02 & 2.72 & 0.01904 & 0.13 & -0.17 & 0.53 & 0.01712 & 4.25 & 4.08 & 4.49 & 0.01256 \\
\hline 31.61 & 7.33 & 2.45 & 2.18 & 2.80 & 0.01102 & 0.17 & -0.14 & 0.47 & 0.01002 & 4.23 & 4.00 & 4.45 & 0.00673 \\
\hline 31.61 & 12.67 & 2.64 & 2.27 & 2.96 & 0.01484 & 0.06 & -0.15 & 0.43 & 0.01201 & 4.27 & 4.06 & 4.50 & 0.00960 \\
\hline 31.61 & 13.45 & 2.58 & 2.31 & 2.92 & 0.02177 & 0.14 & -0.22 & 0.56 & 0.02145 & 4.28 & 4.08 & 4.48 & 0.01192 \\
\hline 31.61 & 14.22 & 2.36 & 1.96 & 2.64 & 0.01867 & 0.31 & -0.02 & 0.67 & 0.01863 & 4.28 & 4.07 & 4.48 & 0.01175 \\
\hline 31.61 & 15.00 & 2.34 & 1.96 & 2.83 & 0.02229 & 0.01 & -0.40 & 0.29 & 0.01649 & 4.31 & 4.15 & 4.48 & 0.00970 \\
\hline 31.61 & 15.78 & 2.59 & 2.36 & 2.81 & 0.01404 & 0.14 & -0.09 & 0.41 & 0.01490 & 4.42 & 4.25 & 4.63 & 0.01143 \\
\hline 31.61 & 16.55 & 2.58 & 2.36 & 2.95 & 0.01645 & 0.17 & -0.06 & 0.41 & 0.01485 & 4.51 & 4.34 & 4.74 & 0.01062 \\
\hline 31.61 & 17.33 & 2.55 & 2.28 & 3.00 & 0.00947 & 0.14 & -0.14 & 0.43 & 0.00829 & 4.51 & 4.19 & 4.69 & 0.00679 \\
\hline 31.67 & 2.67 & 2.35 & 2.13 & 2.55 & 0.03766 & 0.01 & -0.23 & 0.23 & 0.03754 & 4.43 & 4.30 & 4.54 & 0.02248 \\
\hline 31.67 & 12.67 & 2.71 & 2.48 & 2.85 & 0.03765 & -0.01 & -0.35 & 0.16 & 0.04496 & 4.29 & 4.10 & 4.49 & 0.03052 \\
\hline
\end{tabular}




\begin{tabular}{|c|c|c|c|c|c|c|c|c|c|c|c|c|c|}
\hline 31.72 & 2.67 & 2.31 & 2.16 & 2.47 & 0.03205 & 0.06 & -0.11 & 0.19 & 0.02977 & 4.40 & 4.28 & 4.54 & 0.02711 \\
\hline 31.72 & 12.67 & 2.66 & 2.47 & 2.97 & 0.04684 & 0.03 & -0.10 & 0.28 & 0.04015 & 4.25 & 4.13 & 4.42 & 0.03125 \\
\hline 31.78 & 2.67 & 2.56 & 2.27 & 2.76 & 0.06053 & -0.01 & -0.13 & 0.15 & 0.02879 & 4.51 & 4.41 & 4.69 & 0.02507 \\
\hline 31.78 & 12.67 & 2.85 & 2.57 & 3.05 & 0.04874 & 0.01 & -0.19 & 0.14 & 0.03227 & 4.31 & 4.14 & 4.47 & 0.03170 \\
\hline 31.84 & 2.67 & 2.29 & 2.03 & 2.48 & 0.04658 & -0.03 & -0.12 & 0.10 & 0.02088 & 4.36 & 4.24 & 4.45 & 0.02153 \\
\hline 31.84 & 12.67 & 2.67 & 2.43 & 2.92 & 0.04222 & 0.03 & -0.25 & 0.18 & 0.04343 & 4.20 & 4.00 & 4.57 & 0.04892 \\
\hline 31.89 & 2.67 & 2.86 & 2.66 & 3.14 & 0.04635 & -0.02 & -0.14 & 0.13 & 0.02864 & 4.68 & 4.54 & 4.83 & 0.02772 \\
\hline 31.89 & 12.67 & 3.29 & 3.12 & 3.54 & 0.04334 & 0.09 & -0.12 & 0.29 & 0.04018 & 4.53 & 4.42 & 4.64 & 0.02320 \\
\hline 31.95 & 2.67 & 2.85 & 2.64 & 3.17 & 0.01080 & 0.00 & -0.24 & 0.31 & 0.01017 & 4.64 & 4.47 & 4.88 & 0.00770 \\
\hline 31.95 & 3.45 & 3.01 & 2.65 & 3.36 & 0.01584 & 0.29 & -0.18 & 0.54 & 0.01752 & 4.58 & 4.39 & 4.76 & 0.00917 \\
\hline 31.95 & 4.22 & 3.07 & 2.79 & 3.51 & 0.01185 & 0.41 & 0.11 & 0.69 & 0.01212 & 4.47 & 4.25 & 4.62 & 0.00872 \\
\hline 31.95 & 5.00 & 2.80 & 2.41 & 3.18 & 0.01474 & 0.50 & 0.29 & 0.73 & 0.01108 & 4.37 & 4.23 & 4.53 & 0.00753 \\
\hline 31.95 & 5.78 & 2.69 & 2.29 & 3.12 & 0.01858 & 0.26 & -0.20 & 0.67 & 0.02694 & 4.35 & 4.14 & 4.54 & 0.00824 \\
\hline 31.95 & 6.55 & 3.06 & 2.69 & 3.39 & 0.01545 & 0.11 & -0.16 & 0.53 & 0.01483 & 4.46 & 4.15 & 4.73 & 0.01080 \\
\hline 31.95 & 7.33 & 3.07 & 2.73 & 3.40 & 0.01646 & 0.22 & -0.05 & 0.48 & 0.01339 & 4.46 & 4.22 & 4.74 & 0.00997 \\
\hline 31.95 & 12.67 & 3.26 & 2.98 & 3.55 & 0.01208 & 0.09 & -0.25 & 0.39 & 0.01221 & 4.51 & 4.32 & 4.70 & 0.00861 \\
\hline 31.95 & 13.45 & 3.20 & 2.86 & 3.55 & 0.01504 & 0.14 & -0.15 & 0.55 & 0.01488 & 4.56 & 4.29 & 4.80 & 0.01052 \\
\hline 31.95 & 14.22 & 2.81 & 2.46 & 3.27 & 0.01807 & 0.40 & 0.01 & 0.71 & 0.01428 & 4.54 & 4.35 & 4.74 & 0.00859 \\
\hline 31.95 & 15.00 & 2.64 & 2.30 & 2.92 & 0.01378 & 0.01 & -0.39 & 0.56 & 0.01831 & 4.52 & 4.29 & 4.75 & 0.00903 \\
\hline 31.95 & 15.78 & 2.87 & 2.46 & 3.32 & 0.02543 & -0.04 & -0.32 & 0.33 & 0.01555 & 4.58 & 4.40 & 4.81 & 0.01033 \\
\hline 31.95 & 16.55 & 3.25 & 2.96 & 3.56 & 0.01303 & 0.12 & -0.23 & 0.48 & 0.01296 & 4.76 & 4.54 & 4.98 & 0.01027 \\
\hline 31.95 & 17.33 & 3.16 & 2.88 & 3.53 & 0.01562 & 0.13 & -0.18 & 0.39 & 0.01204 & 4.78 & 4.60 & 5.09 & 0.01035 \\
\hline 32.02 & 7.33 & 3.06 & 2.74 & 3.32 & 0.05290 & 0.25 & 0.07 & 0.38 & 0.02751 & 4.50 & 4.37 & 4.71 & 0.03059 \\
\hline 32.02 & 17.33 & 3.15 & 2.87 & 3.30 & 0.04239 & 0.10 & -0.06 & 0.32 & 0.02910 & 4.77 & 4.64 & 4.86 & 0.01535 \\
\hline 32.10 & 7.33 & 3.11 & 2.89 & 3.43 & 0.04200 & 0.23 & -0.09 & 0.41 & 0.04335 & 4.52 & 4.38 & 4.65 & 0.02753 \\
\hline 32.10 & 17.33 & 3.26 & 3.04 & 3.54 & 0.03602 & 0.15 & 0.04 & 0.26 & 0.01798 & 4.85 & 4.74 & 5.00 & 0.02409 \\
\hline 32.18 & 7.33 & 3.11 & 2.90 & 3.38 & 0.04390 & 0.23 & -0.08 & 0.43 & 0.04508 & 4.49 & 4.40 & 4.55 & 0.01498 \\
\hline 32.18 & 17.33 & 3.29 & 3.05 & 3.58 & 0.04343 & 0.22 & -0.07 & 0.51 & 0.04660 & 4.86 & 4.75 & 4.97 & 0.02049 \\
\hline 32.25 & 7.33 & 3.19 & 2.94 & 3.40 & 0.03924 & 0.22 & 0.00 & 0.41 & 0.03135 & 4.47 & 4.22 & 4.57 & 0.02780 \\
\hline 32.25 & 17.33 & 3.33 & 3.22 & 3.49 & 0.02643 & 0.23 & 0.03 & 0.40 & 0.03582 & 4.80 & 4.57 & 4.92 & 0.02566 \\
\hline 32.33 & 7.33 & 3.16 & 3.00 & 3.36 & 0.03129 & 0.17 & 0.00 & 0.34 & 0.03794 & 4.52 & 4.33 & 4.72 & 0.02901 \\
\hline 32.33 & 17.33 & 3.26 & 3.11 & 3.37 & 0.02890 & 0.17 & 0.06 & 0.36 & 0.03220 & 4.83 & 4.72 & 4.90 & 0.01904 \\
\hline 32.41 & 2.67 & 2.82 & 2.41 & 3.15 & 0.01512 & 0.06 & -0.16 & 0.31 & 0.01154 & 4.54 & 4.32 & 4.70 & 0.00890 \\
\hline 32.41 & 3.45 & 3.00 & 2.68 & 3.27 & 0.01025 & 0.21 & -0.02 & 0.47 & 0.00923 & 4.48 & 4.31 & 4.68 & 0.00645 \\
\hline 32.41 & 4.22 & 3.09 & 2.72 & 3.50 & 0.01228 & 0.20 & -0.11 & 0.51 & 0.01130 & 4.55 & 4.35 & 4.84 & 0.00800 \\
\hline 32.41 & 5.00 & 3.15 & 2.82 & 3.48 & 0.01107 & 0.32 & -0.06 & 0.73 & 0.01322 & 4.57 & 4.34 & 4.80 & 0.00744 \\
\hline 32.41 & 5.78 & 3.01 & 2.47 & 3.52 & 0.02297 & 0.39 & -0.34 & 0.72 & 0.01605 & 4.51 & 4.25 & 4.78 & 0.00740 \\
\hline 32.41 & 6.55 & 3.15 & 2.69 & 3.57 & 0.01667 & 0.06 & -0.36 & 0.38 & 0.01385 & 4.53 & 4.31 & 4.75 & 0.00744 \\
\hline 32.41 & 7.33 & 3.14 & 2.84 & 3.45 & 0.01761 & 0.17 & -0.06 & 0.55 & 0.01536 & 4.53 & 4.27 & 4.73 & 0.01064 \\
\hline 32.41 & 12.67 & 3.30 & 2.93 & 3.61 & 0.01481 & 0.07 & -0.20 & 0.29 & 0.01238 & 4.43 & 4.14 & 4.66 & 0.01092 \\
\hline 32.41 & 13.45 & 3.23 & 2.90 & 3.51 & 0.01140 & 0.15 & -0.10 & 0.51 & 0.01169 & 4.50 & 4.28 & 4.73 & 0.00849 \\
\hline 32.41 & 14.22 & 3.31 & 2.96 & 3.68 & 0.01141 & 0.16 & -0.22 & 0.53 & 0.01245 & 4.58 & 4.33 & 4.87 & 0.00853 \\
\hline 32.41 & 15.00 & 3.16 & 2.57 & 3.60 & 0.02209 & 0.32 & -0.15 & 0.64 & 0.01452 & 4.67 & 4.46 & 4.91 & 0.00765 \\
\hline 32.41 & 15.78 & 2.91 & 2.47 & 3.36 & 0.01612 & 0.20 & -0.26 & 0.78 & 0.02348 & 4.70 & 4.50 & 4.93 & 0.00724 \\
\hline 32.41 & 16.55 & 3.36 & 3.07 & 3.66 & 0.01020 & 0.14 & -0.23 & 0.48 & 0.01309 & 4.82 & 4.60 & 5.08 & 0.00790 \\
\hline 32.41 & 17.33 & 3.31 & 3.02 & 3.59 & 0.01647 & 0.15 & -0.09 & 0.40 & 0.01308 & 4.86 & 4.64 & 5.05 & 0.01193 \\
\hline 32.48 & 2.67 & 2.87 & 2.79 & 2.91 & 0.04002 & -0.01 & -0.06 & 0.02 & 0.02708 & 4.53 & 4.47 & 4.61 & 0.04140 \\
\hline 32.48 & 12.67 & 3.28 & 3.06 & 3.45 & 0.11476 & 0.13 & 0.00 & 0.27 & 0.07708 & 4.40 & 4.31 & 4.54 & 0.07005 \\
\hline 32.56 & 2.67 & 2.85 & 2.82 & 2.88 & 0.03215 & 0.09 & 0.05 & 0.12 & 0.03575 & 4.49 & 4.44 & 4.54 & 0.04890 \\
\hline 32.56 & 12.67 & 3.35 & 3.27 & 3.43 & 0.07870 & -0.12 & -0.14 & -0.10 & 0.01965 & 4.39 & 4.38 & 4.40 & 0.01310 \\
\hline 32.64 & 2.67 & 2.73 & 2.62 & 2.82 & 0.05896 & 0.04 & 0.00 & 0.06 & 0.01627 & 4.53 & 4.44 & 4.60 & 0.04824 \\
\hline 32.64 & 12.67 & 3.17 & 3.04 & 3.27 & 0.06605 & 0.06 & -0.01 & 0.11 & 0.03714 & 4.40 & 4.27 & 4.58 & 0.09209 \\
\hline 32.71 & 2.67 & 2.84 & 2.75 & 2.93 & 0.08955 & 0.16 & 0.12 & 0.21 & 0.04595 & 4.39 & 4.33 & 4.46 & 0.06165 \\
\hline 32.71 & 12.67 & 3.29 & 3.29 & 3.30 & 0.00525 & -0.11 & -0.14 & -0.09 & 0.02760 & 4.39 & 4.37 & 4.41 & 0.01905 \\
\hline 32.79 & 2.67 & 2.88 & 2.83 & 2.94 & 0.05675 & 0.04 & -0.02 & 0.10 & 0.05970 & 4.47 & 4.42 & 4.51 & 0.04040 \\
\hline 32.79 & 12.67 & 3.44 & 3.43 & 3.44 & 0.00690 & 0.18 & 0.17 & 0.20 & 0.01510 & 4.42 & 4.35 & 4.48 & 0.06960 \\
\hline 32.87 & 2.67 & 2.56 & 2.31 & 2.88 & 0.01040 & 0.08 & -0.20 & 0.37 & 0.00974 & 4.62 & 4.41 & 4.85 & 0.00727 \\
\hline 32.87 & 3.45 & 2.63 & 2.34 & 2.89 & 0.01465 & 0.24 & -0.07 & 0.59 & 0.02051 & 4.59 & 4.38 & 4.81 & 0.01166 \\
\hline 32.87 & 4.22 & 2.80 & 2.58 & 3.06 & 0.01552 & 0.39 & 0.14 & 0.66 & 0.01408 & 4.50 & 4.37 & 4.70 & 0.00842 \\
\hline
\end{tabular}




\begin{tabular}{|c|c|c|c|c|c|c|c|c|c|c|c|c|c|}
\hline 32.87 & 5.00 & 2.59 & 2.12 & 2.95 & 0.02436 & 0.45 & 0.13 & 0.74 & 0.01918 & 4.46 & 4.34 & 4.66 & 0.00936 \\
\hline 32.87 & 5.78 & 2.60 & 2.22 & 3.16 & 0.02379 & 0.08 & -0.20 & 0.32 & 0.01459 & 4.44 & 4.25 & 4.57 & 0.00885 \\
\hline 32.87 & 6.55 & 2.80 & 2.47 & 3.09 & 0.01830 & 0.14 & -0.13 & 0.50 & 0.01602 & 4.44 & 4.23 & 4.61 & 0.01044 \\
\hline 32.87 & 7.33 & 2.74 & 2.46 & 3.06 & 0.01559 & 0.20 & -0.04 & 0.45 & 0.01392 & 4.44 & 4.23 & 4.67 & 0.01090 \\
\hline 32.87 & 12.67 & 2.98 & 2.51 & 3.32 & 0.01352 & 0.00 & -0.33 & 0.32 & 0.01138 & 4.54 & 4.32 & 4.72 & 0.00731 \\
\hline 32.87 & 13.45 & 2.98 & 2.63 & 3.21 & 0.01587 & 0.04 & -0.28 & 0.45 & 0.01879 & 4.58 & 4.39 & 4.78 & 0.01249 \\
\hline 32.87 & 14.22 & 2.78 & 2.42 & 3.22 & 0.02395 & 0.23 & -0.09 & 0.57 & 0.01900 & 4.63 & 4.44 & 4.82 & 0.01032 \\
\hline 32.87 & 15.00 & 2.48 & 2.07 & 2.98 & 0.02477 & -0.01 & -0.33 & 0.49 & 0.02374 & 4.69 & 4.50 & 4.87 & 0.01035 \\
\hline 32.87 & 15.78 & 2.88 & 2.52 & 3.18 & 0.01864 & -0.07 & -0.37 & 0.18 & 0.01753 & 4.71 & 4.57 & 4.84 & 0.00823 \\
\hline 32.87 & 16.55 & 2.92 & 2.64 & 3.19 & 0.01515 & 0.06 & -0.16 & 0.37 & 0.01693 & 4.79 & 4.59 & 5.02 & 0.01073 \\
\hline 32.87 & 17.33 & 2.85 & 2.56 & 3.14 & 0.01341 & 0.07 & -0.19 & 0.28 & 0.01228 & 4.82 & 4.68 & 4.99 & 0.00779 \\
\hline 32.95 & 2.67 & 2.56 & 2.28 & 2.83 & 0.01115 & 0.31 & -0.03 & 0.64 & 0.00997 & 4.66 & 4.50 & 4.84 & 0.00622 \\
\hline 32.95 & 3.45 & 2.68 & 2.42 & 2.94 & 0.01485 & 0.23 & -0.05 & 0.42 & 0.01306 & 4.60 & 4.36 & 4.80 & 0.01116 \\
\hline 32.95 & 4.22 & 2.74 & 2.35 & 2.99 & 0.01585 & 0.40 & -0.03 & 0.71 & 0.01833 & 4.51 & 4.38 & 4.69 & 0.00893 \\
\hline 32.95 & 5.00 & 2.44 & 2.13 & 2.88 & 0.02122 & 0.23 & -0.32 & 0.66 & 0.02836 & 4.50 & 4.32 & 4.69 & 0.00975 \\
\hline 32.95 & 5.78 & 2.67 & 2.28 & 2.99 & 0.01622 & 0.08 & -0.20 & 0.35 & 0.01592 & 4.50 & 4.32 & 4.68 & 0.00896 \\
\hline 32.95 & 6.55 & 2.71 & 2.25 & 2.98 & 0.01766 & 0.16 & -0.09 & 0.49 & 0.01726 & 4.52 & 4.27 & 4.71 & 0.01111 \\
\hline 32.95 & 7.33 & 2.72 & 2.30 & 3.06 & 0.01232 & 0.17 & -0.22 & 0.62 & 0.01024 & 4.51 & 4.23 & $\begin{array}{ll}4.77 \\
\end{array}$ & 0.00660 \\
\hline 32.95 & 12.67 & 2.92 & 2.51 & 3.32 & 0.01340 & 0.10 & -0.12 & 0.41 & 0.00952 & 4.61 & 4.38 & 4.80 & 0.00777 \\
\hline 32.95 & 13.45 & 2.92 & 2.62 & 3.28 & 0.01936 & 0.10 & -0.21 & 0.50 & 0.01847 & 4.66 & 4.42 & 4.86 & 0.01203 \\
\hline 32.95 & 14.22 & 2.57 & 2.16 & 3.03 & 0.02360 & 0.29 & -0.13 & 0.59 & 0.01909 & 4.74 & 4.59 & 4.88 & 0.00762 \\
\hline 32.95 & 15.00 & 2.59 & 2.00 & 3.05 & 0.02792 & -0.03 & -0.29 & 0.26 & 0.01470 & 4.79 & 4.66 & 4.98 & 0.00925 \\
\hline 32.95 & 15.78 & 2.88 & 2.59 & 3.27 & 0.01616 & 0.12 & -0.18 & 0.48 & 588 & 4.82 & 4.55 & 5.00 & 014 \\
\hline 32.95 & 16.55 & 2.84 & 2.49 & 3.26 & 0.01764 & 0.14 & -0.14 & 0.47 & 0.01541 & 4.95 & 4.74 & 5.19 & 0.01077 \\
\hline 32.95 & 17.33 & 2.83 & 2.51 & 3.18 & 0.01005 & 0.11 & -0.23 & 0.37 & 0.00837 & 4.95 & 4.74 & 5.16 & 0.00633 \\
\hline 33.31 & 7.33 & 2.71 & 2.45 & 2.88 & 0.03164 & 0.17 & -0.10 & 0.40 & 0.02966 & 4.51 & 4.38 & 4.69 & 0.02031 \\
\hline 33.31 & 17.33 & 2.75 & 2.47 & 2.98 & 0.03197 & 0.10 & -0.24 & 0.30 & 0.03072 & 4.94 & 4.77 & 5.03 & 0.01507 \\
\hline 33.66 & 7.33 & 2.72 & 2.30 & 3.27 & 0.06401 & 0.25 & -0.06 & 0.69 & 0.04279 & 5.29 & 4.30 & 5.97 & 0.14429 \\
\hline 33.66 & 17.33 & 2.74 & 2.24 & 3.29 & 0.07330 & -0.01 & -0.24 & 0.25 & 0.03754 & 5.72 & 4.76 & 6.51 & 0.14420 \\
\hline 34.02 & 7.33 & 2.47 & 2.12 & 2.68 & 0.04053 & 0.28 & 0.07 & 0.51 & 0.02896 & 5.96 & 5.80 & 6.20 & 0.02109 \\
\hline 34.02 & 17.33 & 2.47 & 2.21 & 2.67 & 0.03175 & -0.09 & -0.32 & 0.12 & 0.02728 & 6.38 & 6.24 & 6.53 & 0.01724 \\
\hline 34.37 & 7.33 & 2.48 & 2.19 & 2.66 & 0.03261 & 0.26 & 0.04 & 0.54 & 0.03255 & 5.94 & 5.85 & 6.05 & 0.01396 \\
\hline 34.37 & 17.33 & 2.47 & 2.10 & 2.70 & 0.03422 & -0.04 & -0.49 & 0.21 & 0.03794 & 6.36 & 6.12 & 6.52 & 0.02247 \\
\hline 34.73 & 7.33 & 2.44 & 2.15 & 2.76 & 0.03332 & 0.26 & 0.03 & 0.48 & 0.02670 & 5.96 & 5.75 & 6.08 & 0.01675 \\
\hline 34.73 & 17.33 & 2.42 & 2.14 & 2.72 & 0.03788 & -0.08 & -0.33 & 0.18 & 0.02627 & 6.39 & 6.16 & 6.56 & 0.02372 \\
\hline 35.08 & 2.67 & 2.27 & 1.23 & 3.68 & 0.00640 & 0.12 & -0.93 & 0.98 & 0.00657 & 6.15 & 5.52 & 6.79 & 0.00315 \\
\hline 35.08 & 3.45 & 2.43 & 0.96 & 4.04 & 0.01208 & 0.54 & -0.76 & 2.00 & 0.01226 & 6.05 & 5.42 & 6.61 & 0.00539 \\
\hline 35.08 & 4.22 & 2.59 & 1.10 & 4.04 & 0.01163 & 0.65 & -0.82 & 1.93 & 0.01152 & 5.96 & 5.43 & 6.48 & 0.00524 \\
\hline 35.08 & 5.00 & 2.43 & 0.98 & 4.09 & 0.01315 & 0.68 & -0.78 & 1.98 & 0.01265 & 5.90 & 5.23 & 6.49 & 0.00548 \\
\hline 35.08 & 5.78 & 2.34 & 1.12 & 4.04 & 0.01336 & 0.26 & -0.80 & 1.62 & 178 & 5.90 & 5.24 & 6.49 & 583 \\
\hline 35.08 & 6.55 & 2.57 & 1.01 & 4.07 & 0.01283 & 0.32 & -0.83 & 1.82 & 0.01251 & 5.87 & 5.37 & 6.55 & 0.00543 \\
\hline 35.08 & 7.33 & 2.45 & 0.79 & 4.68 & 0.00682 & 0.30 & -0.74 & 1.98 & 0.00696 & 5.92 & 5.17 & 6.58 & 0.00321 \\
\hline 35.08 & 12.67 & 2.68 & 1.40 & 3.85 & 0.00611 & -0.08 & -1.00 & 1.00 & 0.00590 & 5.94 & 5.19 & 6.60 & 0.00317 \\
\hline 35.08 & 13.45 & 2.65 & 1.72 & 3.47 & 0.01015 & 0.00 & -0.97 & 0.99 & 0.01057 & 5.98 & 5.37 & 6.52 & 0.00543 \\
\hline 35.08 & 14.22 & 2.40 & 1.52 & 3.47 & 0.01050 & 0.09 & -0.99 & 0.98 & 0.01112 & 6.07 & 5.36 & 6.55 & 0.00529 \\
\hline 35.08 & 15.00 & 2.15 & 1.50 & 3.11 & 0.00981 & -0.08 & -0.96 & 0.99 & 0.01098 & 6.15 & 5.55 & 6.67 & 0.00479 \\
\hline 35.08 & 15.78 & 2.44 & 1.52 & 3.42 & 0.01067 & -0.11 & -1.00 & 0.86 & 0.01089 & 6.18 & 5.61 & 6.85 & 0.00505 \\
\hline 35.08 & 16.55 & 2.49 & 1.52 & 3.48 & 0.01128 & 0.01 & -0.96 & 0.97 & 0.01130 & 6.26 & 5.61 & 6.69 & 0.00553 \\
\hline 35.08 & 17.33 & 2.42 & 1.38 & 3.50 & 0.00597 & -0.09 & -1.23 & 0.98 & 0.00596 & 6.37 & 5.49 & 6.97 & 0.00313 \\
\hline 35.21 & 2.67 & 0.25 & -0.74 & 1.24 & 0.01457 & 0.10 & -0.87 & 0.99 & 0.01386 & 7.40 & 6.97 & 7.90 & 0.00714 \\
\hline 35.21 & 12.67 & 0.55 & -0.24 & 1.22 & 0.01424 & -0.26 & -0.98 & 0.45 & 0.01476 & 7.31 & 6.88 & 7.78 & 0.00767 \\
\hline 35.35 & 2.67 & 0.27 & -0.69 & 1.25 & 0.01569 & 0.11 & -0.87 & 0.93 & 0.01336 & 7.33 & 6.83 & 7.75 & 0.00678 \\
\hline 35.35 & 12.67 & 0.64 & -0.24 & 1.25 & 0.01440 & -0.25 & -1.00 & 0.49 & 0.01435 & 7.24 & 6.66 & 7.67 & 0.00734 \\
\hline 35.49 & 2.67 & 0.25 & -0.76 & 1.12 & 0.01593 & 0.10 & -0.80 & 0.98 & 0.01373 & 7.37 & 6.90 & 7.80 & 0.00685 \\
\hline 35.49 & 12.67 & 0.53 & -0.25 & 1.23 & 0.01492 & -0.30 & -0.98 & 0.50 & 0.01456 & 7.25 & 6.77 & 7.61 & 0.00758 \\
\hline 35.62 & 2.67 & 0.20 & -0.80 & 1.22 & 0.01450 & 0.09 & -0.81 & 0.96 & 0.01393 & 7.33 & 6.93 & 7.69 & 0.00660 \\
\hline 35.62 & 12.67 & 0.59 & -0.24 & 1.23 & 0.01405 & -0.28 & -0.99 & 0.50 & 0.01486 & 7.22 & 6.80 & 7.80 & 0.00731 \\
\hline 35.76 & 2.67 & 0.31 & -0.70 & 1.20 & 0.01488 & 0.16 & -0.67 & 0.94 & 0.01386 & 7.36 & 6.91 & 7.77 & 0.00638 \\
\hline 35.76 & 12.67 & 0.65 & -0.14 & 1.25 & 0.01404 & -0.24 & -1.00 & 0.50 & 0.01492 & 7.24 & 6.85 & 7.73 & 0.00693 \\
\hline
\end{tabular}


Water Velocity Measurements on an ESBS at John Day Dam

\begin{tabular}{|l|l|l|l|l|l|l|l|l|l|l|l|l|l|}
\hline 35.89 & 2.67 & 0.26 & -0.96 & 1.45 & 0.00565 & 0.12 & -0.99 & 1.25 & 0.00521 & 7.38 & 6.89 & 7.87 & 0.00229 \\
\hline 35.89 & 3.45 & 0.35 & -0.79 & 1.61 & 0.01140 & 0.13 & -1.01 & 1.28 & 0.01189 & 7.31 & 6.90 & 7.83 & 0.00457 \\
\hline 35.89 & 4.22 & 0.48 & -0.77 & 1.61 & 0.01069 & 0.26 & -0.86 & 1.35 & 0.01152 & 7.28 & 6.82 & 7.69 & 0.00462 \\
\hline 35.89 & 5.00 & 0.42 & -0.55 & 1.51 & 0.01096 & 0.24 & -1.03 & 1.28 & 0.01061 & 7.29 & 6.81 & 7.86 & 0.00449 \\
\hline 35.89 & 5.78 & 0.33 & -0.77 & 1.54 & 0.01205 & 0.11 & -1.00 & 1.25 & 0.01092 & 7.26 & 6.74 & 7.81 & 0.00498 \\
\hline 35.89 & 6.55 & 0.35 & -0.80 & 1.53 & 0.01193 & 0.05 & -1.04 & 1.33 & 0.01122 & 7.24 & 6.78 & 7.69 & 0.00503 \\
\hline 35.89 & 7.33 & 0.27 & -0.97 & 1.77 & 0.00703 & -0.01 & -0.99 & 0.99 & 0.00578 & 7.23 & 6.71 & 7.77 & 0.00297 \\
\hline 35.89 & 12.67 & 0.57 & -0.49 & 1.70 & 0.00573 & -0.25 & -1.30 & 0.74 & 0.00540 & 7.28 & 6.73 & 7.87 & 0.00250 \\
\hline 35.89 & 13.45 & 0.52 & -0.88 & 1.54 & 0.01096 & -0.30 & -1.41 & 0.73 & 0.01123 & 7.27 & 6.76 & 7.78 & 0.00450 \\
\hline 35.89 & 14.22 & 0.33 & -0.69 & 1.53 & 0.01060 & -0.35 & -1.43 & 0.74 & 0.01076 & 7.33 & 6.74 & 7.78 & 0.00470 \\
\hline 35.89 & 15.00 & 0.27 & -0.79 & 1.35 & 0.01060 & -0.41 & -1.48 & 0.67 & 0.00987 & 7.42 & 6.76 & 7.92 & 0.00466 \\
\hline 35.89 & 15.78 & 0.47 & -0.42 & 1.73 & 0.01086 & -0.53 & -1.50 & 0.49 & 0.00987 & 7.45 & 6.89 & 7.95 & 0.00473 \\
\hline 35.89 & 16.55 & 0.67 & -0.38 & 1.97 & 0.01115 & -0.59 & -1.54 & 0.62 & 0.01106 & 7.55 & 6.96 & 8.11 & 0.00525 \\
\hline 35.89 & 17.33 & 0.68 & -0.45 & 1.98 & 0.00703 & -0.62 & -1.49 & 0.49 & 0.00628 & 7.64 & 7.01 & 8.30 & 0.00304 \\
\hline
\end{tabular}




\section{Appendix B}

\section{Spherical Coordinates for Velocity Data at $155 \mathrm{MW}$ Turbine Load}




\section{Appendix B}

\section{Spherical Coordinates for Velocity Data at 155 MW Turbine Load}

The table in this appendix lists spherical coordinates for velocity data collected at locations along the bar screen surface of the ESBS, where Rho is the magnitude flow velocity (ft/sec) and phi and theta are angles of the flow off the positive polar axis and equatorial planes, respectively, at $155 \mathrm{MW}$ turbine load.

\begin{tabular}{|c|c|c|c|c|c|c|c|c|c|c|c|c|c|}
\hline \multicolumn{2}{|c|}{ Location (ft.) } & \multicolumn{4}{|c|}{ Rho (magnitude velocity) } & \multicolumn{4}{|c|}{ Phi } & \multicolumn{4}{|c|}{ Theta } \\
\hline Vertical & Horizontal & Mean & Min & Max & Stderr & Mean & Min & Max & tderr & Mean & Min & Max & Stderr \\
\hline-0.02 & 2.67 & 7.75 & 7.02 & 8.11 & .01440 & 128.90 & 124.43 & 136.40 & .20179 & 4.83 & -3.27 & 13.20 & 0.20734 \\
\hline-0.02 & 3.45 & 29 & 6.76 & .90 & 02112 & 127.69 & 120.28 & 137.68 & .38429 & 4.22 & -3.92 & 16.61 & 38886 \\
\hline-0.02 & 4.22 & 86 & 6.15 & 7.35 & 02169 & 127.67 & 120.12 & 135.64 & 31089 & 6.27 & -4.66 & & \\
\hline-0.02 & .00 & 16 & 5.65 & 3.64 & & 127.67 & 116.98 & 136.15 & & .07 & -5.11 & 20.63 & \\
\hline-0.02 & 5.78 & 1 & 6.14 & 7.63 & .03068 & 128.65 & 115.32 & 136.63 & 147 & 8.20 & -1.86 & 20.00 & 9831 \\
\hline-0.02 & 6.55 & 42 & 6.71 & 8.16 & & 128.06 & 118.70 & 136.10 & & & -3.33 & & \\
\hline-0.02 & .33 & 27 & 6.77 & & & 124.90 & 117.57 & 130.71 & & 0.79 & -5.56 & 5.94 & \\
\hline-0.02 & 12.67 & 7.47 & 6.97 & 7.95 & & 124.88 & 117.30 & 131.49 & 248 & 10.32 & 3.82 & 14.14 & \\
\hline-0.02 & 13.45 & 7 & 6.61 & & & 124 & 115.81 & 133.33 & & & 1.17 & 17.63 & \\
\hline-0.02 & 14.22 & 01 & 5.97 & & & 128.53 & 119.38 & 138.54 & & 22 & -7.65 & 17.63 & \\
\hline-0.02 & 15.00 & 5 & 5.93 & & & 131.76 & 123.85 & 141.45 & 588 & -2.57 & -12.40 & 6.94 & \\
\hline-0.02 & 15.78 & 8 & 5.87 & 7. & & 128.28 & 115.17 & 138.67 & & 4.31 & -9.59 & 13.13 & \\
\hline-0.02 & 16.55 & 9 & 6.83 & & & & 117.45 & 133.54 & & 93 & 2.86 & 68 & \\
\hline-0.02 & 17.33 & 6 & 7.00 & & & 125.07 & 114.06 & 133.60 & & 9.90 & -0.06 & 22.55 & \\
\hline & 3 & & & & & & 114.71 & 132.31 & & & & 67 & \\
\hline 0 & 17.33 & 5 & 4.73 & & & & 116.61 & 133.45 & & 10.06 & 5.76 & 14.16 & \\
\hline 0. & 7.33 & & 4.0 & & & & 105.69 & 117.74 & & 4.01 & 0.78 & 9.08 & \\
\hline 0 & .33 & & & & & & 103 & 131.33 & & & 11.68 & 21.33 & \\
\hline 0. & 7.33 & 4 & 4.10 & & & & 105 & 115.28 & & 3.79 & 0.60 & 7.57 & \\
\hline 0. & 17.33 & & 3.6 & & & & 104.65 & 114.46 & & & & 23.53 & \\
\hline & & & & & & & & 89 & & & 10 & & \\
\hline 1.27 & 17.33 & 3 & 3.61 & & & & 10 & 118.08 & & 15.43 & 7.40 & 22.84 & \\
\hline 1.60 & 732 & & & & & & & 114.62 & & 4.09 & -0.72 & 7.67 & \\
\hline & .33 & & & & & & & 120.75 & & 15.29 & 19 & & \\
\hline 1.92 & 2.67 & 8 & 3.90 & & & 11 & $10 s$ & 124.59 & & 5.13 & -3.25 & \begin{tabular}{|l}
14.72 \\
\end{tabular} & \\
\hline & 3.45 & & & & & & & 124.79 & & 4.91 & & & \\
\hline & 4.22 & & & & & & & 125.26 & & & -1.23 & & \\
\hline 1.92 & 5.00 & 4 & 3. & & & 11 & 108 & 125.09 & & 12.12 & 2.79 & 26 & \\
\hline 1.9 & 5.78 & & & & & 112.03 & 107.02 & 118.38 & & 7.86 & -1.33 & 16.22 & \\
\hline & & & & & & & & & & & 0.41 & & \\
\hline 1. & 7.33 & 4 & 3.95 & 4 & & 10 & 102 & 116.46 & & 4.62 & -2.15 & 26 & 171 \\
\hline 1.92 & 12.67 & & 3.83 & & & & & 121.82 & & 9.45 & 0.33 & 16.01 & \\
\hline 1.9 & 13.45 & & 4. & & & & 10 & 121.97 & & 41 & 2.55 & 14 & \\
\hline & 14.22 & & 3.90 & & & 110.09 & 105.69 & 115.48 & & 73 & 5.24 & 16.49 & \\
\hline 1.92 & 15.00 & & 3.3 & & & & 104.57 & 118.46 & & 8.73 & -1.89 & 16.77 & \\
\hline 1.92 & 15.78 & & 3.69 & 4. & 19 & 115 & 108 & 121.58 & 64 & 3.78 & -2.64 & 11.54 & \\
\hline 1.92 & 16.55 & & 3.65 & & & 117.48 & 111.41 & 126.55 & 147 & 9.07 & 0.55 & 16.88 & 654 \\
\hline 1.92 & 17.33 & & 3.42 & & & & & 123.14 & & 15.27 & 5.40 & 23.27 & \\
\hline 2.67 & 2.67 & 3.36 & 2.51 & 4.54 & 0.0 & 111.36 & 101.43 & 126.76 & 664 & 3.94 & -4.27 & 14.21 & \\
\hline 2.67 & 12.67 & & 2.64 & & & 107.50 & 94.07 & 119.21 & 072 & 10.06 & 0.97 & 17.89 & 158 \\
\hline 3.42 & 2.67 & 3.89 & 3.33 & & & & 104.49 & 129.11 & & 4.93 & -3.23 & 14.24 & \\
\hline 3.42 & 12.67 & 3.95 & 3.35 & 4.72 & & 111.54 & 103.08 & 120.79 & 0.24511 & 9.70 & -2.54 & 19.22 & 0.25073 \\
\hline 4.17 & 2.67 & & 3.77 & 4.71 & 0.01241 & 117.17 & 108.99 & 128.44 & 3564 & 4.73 & -2.68 & 16.06 & 0.22871 \\
\hline 4.17 & 12.67 & 4.25 & 3.79 & & & & & 122.13 & & 9.62 & 2.11 & & 0.20554 \\
\hline 4.92 & 2.67 & 4.03 & 3.36 & 4.55 & 0.01937 & 115.97 & 109.00 & 124.55 & 0.24347 & 4.53 & -3.48 & 13.25 & 0.22628 \\
\hline
\end{tabular}




\begin{tabular}{|c|c|c|c|c|c|c|c|c|c|c|c|c|c|}
\hline 4.92 & 12.67 & 4.05 & 3.41 & 4.79 & 0.02151 & 112.42 & 104.01 & 120.15 & 0.23708 & 9.69 & 0.15 & 16.00 & 0.22264 \\
\hline 5.67 & 2.67 & 3.12 & 2.24 & 4.21 & 0.03133 & 98.49 & 81.52 & 122.31 & 0.91454 & 3.28 & -4.32 & 13.41 & 0.24734 \\
\hline 5.67 & 2.67 & 3.30 & 2.35 & 4.09 & 0.02523 & 97.87 & 33.93 & 124.73 & 0.73394 & 9.44 & 0.26 & 18.12 & .22529 \\
\hline 6.41 & 2.67 & 2.99 & 1.80 & 4.44 & 0.00475 & 87.24 & 69.43 & 105.00 & 0.06364 & 1.34 & -22.47 & 32.69 & 0.08315 \\
\hline 6.41 & 3.45 & 3.01 & 2.04 & 4.18 & 0.01636 & 88.35 & 75.76 & 103.54 & 0.25756 & 1.43 & -16.13 & 25.01 & .32030 \\
\hline .41 & 4.22 & 2.91 & 2.00 & 3.83 & 0.01518 & 91.63 & 73.88 & 105.25 & .24938 & 3.42 & -14.18 & 25.77 & 0.32704 \\
\hline 6.41 & 5.00 & 2.93 & 1.65 & 3.88 & 0.01474 & 90.90 & 73.39 & 103.73 & 0.26034 & 5.88 & -14.19 & 28.06 & 0.30158 \\
\hline 6.41 & 5.78 & 2.93 & 1.90 & 4.02 & 0.01502 & 90.31 & 72.85 & 103.61 & 0.25621 & 9.79 & -9.38 & 26.43 & 0.29651 \\
\hline 6.41 & 6.55 & 2.64 & 1.58 & 3.74 & 0.01728 & 89.42 & 67.11 & 113.46 & 0.34878 & 10.13 & -8.91 & 29.61 & 0.31328 \\
\hline 6.41 & 7.33 & 3.30 & 1.96 & 4.51 & 0.00438 & 84.63 & 68.64 & 101.68 & 0.06089 & 4.58 & -13.69 & 21.57 & 0.06960 \\
\hline 6.41 & 12.67 & 3.24 & 2.11 & 4.47 & 0.00345 & 88.75 & 71.45 & 103.55 & 0.05052 & 8.67 & -15.61 & 28.33 & 0.06670 \\
\hline 6.41 & .45 & 3.23 & 2.20 & 4.35 & 0.01 & 88.97 & 76.17 & 105.16 & 0.2 & 9.19 & -8.97 & 26.57 & 0.26217 \\
\hline 6.41 & 14.22 & 3.37 & 2.57 & 4.62 & 0.01453 & 87.10 & 73.90 & 99.80 & 0.18943 & 13.06 & -1.48 & 30.07 & 0.24502 \\
\hline 6.41 & 15.00 & 3.34 & 2.61 & 4.49 & 0.0 & 86.22 & 73.50 & 99.67 & 0.18461 & 16.03 & -0.18 & 33.81 & 0.25430 \\
\hline 6.41 & .78 & 3.01 & 2.01 & 3.95 & 0.0 & 85.90 & 74.28 & 96.80 & 0.1 & 17.46 & -0.54 & 35.39 & 0.27400 \\
\hline 6.41 & 16.55 & 2.84 & 1.82 & 3.76 & 0.01294 & 87.30 & 74.59 & 103.20 & 0.20570 & 11.69 & -6.08 & 30.65 & 0.27008 \\
\hline 6.41 & 17.33 & 3.20 & 1.89 & 4.71 & 0.00477 & 80.36 & 63.48 & 99.42 & 0.06280 & 14.87 & -10.06 & 34.71 & 0.07218 \\
\hline 6.83 & 7.33 & 3.27 & 2.19 & 4.35 & 0.0 & 83.75 & 99 & 98.86 & 0.2 & 3.99 & -15.19 & 20.39 & 0.22957 \\
\hline 6.83 & 17.33 & 3.30 & 2.07 & 4.77 & 0.0 & 77.81 & 64.05 & 96.62 & 0.20707 & 14.82 & -6.74 & 33.32 & 0.24357 \\
\hline 7.25 & 7.33 & 3.42 & 2.18 & 4.81 & 0.0 & 82.62 & 67.77 & 99.73 & 0.1 & 3.83 & -10.06 & 22.20 & 0.23056 \\
\hline 7.25 & 17.33 & 3.46 & 2.24 & 4.78 & 0.0 & 76.13 & 62.22 & 94.87 & & 14.65 & -3.73 & 30.59 & 0.24864 \\
\hline 7.66 & 7.33 & 3.58 & 2.67 & 5.09 & 0.0 & 82.27 & 71.66 & 99.36 & 0.1 & 4.86 & -11.26 & 23.58 & 0.23120 \\
\hline 7.66 & 7.33 & 3.59 & 2.27 & 5.06 & 0.0 & 77.21 & 62.30 & 89.73 & 0.1 & 13.73 & -7.21 & 31.57 & 39 \\
\hline 8.08 & 7.33 & 3.50 & 2.46 & 4.56 & & 83.60 & 70.99 & 102.56 & & 4.81 & -12.93 & 22.82 & 51 \\
\hline 8.08 & 7.33 & 3.64 & 2.48 & 4.88 & 0.0 & 75.81 & 61.75 & 87.29 & & 12.89 & -10.86 & 32.59 & 0.23322 \\
\hline 8.50 & 33 & 3.09 & 2.22 & 4.15 & 1 & 83.60 & 68.41 & 97.94 & & 4.69 & $\begin{array}{l}-17.47 \\
\end{array}$ & 26.33 & .24705 \\
\hline 8.50 & .33 & 3.21 & 1.96 & 4.79 & & 75.59 & 98 & 95.54 & & 14.64 & -8.00 & 37.56 & .25660 \\
\hline 8.85 & 2.67 & 2.87 & 1.77 & 3.92 & 0.0 & 80.36 & 68.00 & 99.46 & 0.0 & -1.67 & -19.68 & 18.74 & 0.11422 \\
\hline 8.85 & 3.45 & 2.76 & 1.68 & 3.77 & & 85.12 & 37 & 97.28 & 78 & 1.17 & -19.25 & 26.67 & 81 \\
\hline 8.85 & 4.22 & 2.71 & 1.67 & 3.77 & 0.1 & 87.41 & 73.41 & 101.65 & 0.2 & 5.76 & -18.65 & 24.77 & 0.28120 \\
\hline 8.85 & 5.00 & 2.46 & 1.40 & 3.34 & 0.0 & 87.87 & 71.17 & 103.74 & 0.2 & 3.78 & -23.77 & 31.89 & 0.36204 \\
\hline 8.85 & 78 & 2.89 & 1.95 & 4.05 & & 85.12 & 23 & 06 & 79 & 0.87 & -14.80 & 16.88 & 345 \\
\hline 8.85 & .55 & 2.93 & 2.09 & 3.94 & 0.0 & 83.53 & 65.56 & 101.32 & 0.2 & 4.08 & -13.12 & 21.48 & 0.26206 \\
\hline 8.85 & 7.33 & 2.94 & 2.02 & 4.02 & & 83.49 & 66.51 & 95.90 & & 4.56 & -14.17 & 21.45 & 335 \\
\hline 8.85 & .67 & 3.03 & 1.82 & 4.18 & & 87.51 & 50 & 02.19 & 26 & 7.09 & -11.53 & 27.35 & 940 \\
\hline 8.85 & .45 & 3.10 & 2.21 & 4.36 & & 85.93 & 80 & 104.76 & & 10.30 & -5.61 & 29.73 & 0.25023 \\
\hline 8.85 & 22 & 2.91 & 1.90 & 3.93 & & 84.78 & 72.18 & 101.10 & 0.1 & 16.65 & -3.73 & 41.84 & 0.28588 \\
\hline 8.85 & .00 & 2.69 & 1.52 & 3.64 & & 84.22 & 67.93 & 99.40 & 36 & 9.71 & -12.64 & 31.42 & 0.27565 \\
\hline 8.85 & .78 & 2.85 & 1.94 & 4.15 & & 81.67 & & 96.84 & & 0.82 & -7.50 & 31.03 & 0.28431 \\
\hline 8.85 & .55 & 2.98 & 1.44 & 4.27 & & 76.52 & & 94.70 & & 4.04 & -14.18 & 33.59 & 0.27482 \\
\hline 8.85 & .33 & 3.00 & 1.98 & 4.32 & & 76.09 & 96 & 91.04 & 21 & 3.96 & -8.10 & 34.28 & .24652 \\
\hline 8.92 & 2.67 & 2.70 & 1.56 & 4.11 & & 84.96 & 65.50 & & & 1.25 & -22.29 & 25.79 & .08455 \\
\hline 8.92 & 3.45 & 2.68 & 1.51 & 3.89 & & 86.69 & 72.98 & 103.56 & & 1.69 & -17.38 & 24.09 & 0.24030 \\
\hline 8.92 & 4.22 & 2.65 & 1.73 & 3.71 & & 91.33 & 73.32 & 111.38 & 0.1 & 4.96 & -20.68 & 27.40 & .26067 \\
\hline 8.92 & 5.00 & 2.49 & 1.60 & 3.53 & & 90.71 & 70.19 & 106.32 & & 8.01 & -16.78 & 29.92 & 0.25791 \\
\hline 8.92 & 5.78 & 2.54 & 1.48 & 3.70 & & 88.90 & 72.10 & 106.60 & & 0.88 & -21.01 & 23.56 & 0.26365 \\
\hline 8.92 & 6.55 & 2.83 & 1.88 & 3.89 & & 87.11 & 69.05 & 105.30 & 414 & 3.80 & -18.04 & 24.65 & 0.22837 \\
\hline 8.92 & 7.33 & 2.91 & 1.67 & 4.12 & & 84.58 & 66.71 & 103.06 & & 5.39 & -18.82 & 25.20 & 0.07589 \\
\hline 8.92 & 2.67 & 2.96 & 1.98 & 4.07 & & 87.73 & 71.87 & 102.60 & 0.0 & 7.78 & -14.55 & 28.05 & 0.07166 \\
\hline 8.92 & 3.45 & 2.93 & 1.99 & 3.85 & & 87.65 & 71.86 & 101.59 & & 7.97 & -14.32 & 28.17 & 0.22562 \\
\hline 8.92 & 22 & 3.07 & 2.01 & 4.02 & & 86.48 & 71.72 & 98.76 & 614 & 3.22 & -6.58 & 33.03 & 0.22753 \\
\hline 8.92 & .00 & 2.62 & 1.60 & 3.70 & & 84.69 & 63.59 & 111.05 & 0.17521 & 12.95 & -12.55 & 37.34 & 0.26059 \\
\hline 8.92 & 15.78 & 2.64 & 1.53 & 3.83 & 0.0 & 84.96 & 69.40 & 101.21 & 0.16461 & 10.08 & -9.78 & 32.03 & 0.24578 \\
\hline 8.92 & 16.55 & 2.83 & 1.76 & 4.16 & & 81.34 & 95 & 100.20 & & 3.89 & -9.33 & 43.97 & 0.23856 \\
\hline 8.92 & 17.33 & 2.88 & 1.61 & 4.36 & 0.00485 & 76.64 & 59.59 & 98.23 & 0.06453 & 14.56 & $\begin{array}{l}-17.57 \\
\end{array}$ & 37.45 & 0.07877 \\
\hline 8.94 & 2.67 & 2.92 & 2.16 & 3.69 & 0.02563 & 80.11 & 69.77 & 92.04 & 0.35317 & -1.26 & -18.41 & 17.82 & 0.54449 \\
\hline 8.94 & 12.67 & 3.06 & 2.27 & 4.15 & 0.02 & 86.10 & 76.70 & 95.09 & 0.2 & 8.04 & -10.16 & 20.26 & 0.39089 \\
\hline 9.02 & 2.67 & 2.80 & 2.18 & 3.42 & & 79.99 & 69.50 & 88.81 & 0.64089 & 2.22 & -12.23 & 19.42 & 0.95085 \\
\hline 9.02 & 12.67 & 3.03 & 2.36 & 3.94 & 0.03820 & 88.46 & 79.68 & 101.34 & 0.48248 & 5.86 & -11.45 & 17.34 & 0.72238 \\
\hline
\end{tabular}




\begin{tabular}{|c|c|c|c|c|c|c|c|c|c|c|c|c|c|}
\hline 9.10 & 2.67 & 3.05 & 2.02 & 4.16 & 0.03467 & 79.02 & 65.87 & 89.60 & 0.39420 & -2.09 & $\begin{array}{l}-17.10 \\
\end{array}$ & 12.94 & 0.55270 \\
\hline 9.10 & 12.67 & 3.25 & 2.53 & 3.93 & 0.01959 & 86.20 & 75.76 & 97.69 & 0.36222 & 8.06 & -7.78 & 18.84 & 0.38073 \\
\hline .19 & 67 & 2.74 & .16 & 3.51 & .03524 & 80.20 & 5.82 & 89.16 & .57834 & -4.11 & -19.29 & 13.93 & .80665 \\
\hline 19 & 2.67 & 2.84 & 2.04 & 3.34 & 0.03262 & 89.32 & 79.69 & 97.87 & 0.53588 & 7.90 & -12.23 & 18.28 & .89504 \\
\hline 9.27 & 2.67 & 2.63 & 1.83 & 3.95 & 0.02688 & 81.67 & 64.90 & 101.78 & 0.50235 & -1.86 & -17.20 & 20.38 & .54593 \\
\hline 9.27 & 2.67 & 2.78 & 1.82 & 3.59 & .02544 & 89.34 & 80.13 & 104.46 & .30266 & 6.43 & -12.57 & 24.75 & .46633 \\
\hline 9.35 & 2.67 & 2.58 & 1.50 & 3.74 & 0.00660 & 81.09 & 65.44 & 101.91 & 0.09612 & -1.65 & -29.04 & 18.58 & 0.12692 \\
\hline 9.35 & 3.45 & 2.48 & 1.58 & 3.33 & 0.01262 & 84.61 & 65.87 & 104.79 & 0.24855 & 0.45 & -21.79 & 20.58 & 0.29280 \\
\hline 9.35 & 4.22 & 2.43 & 1.35 & 3.25 & 0.01490 & 89.25 & 73.89 & 106.13 & 0.26263 & 4.44 & -20.98 & 53.04 & 0.38848 \\
\hline 9.35 & 5.00 & 2.21 & 1.22 & 3.02 & 0.01342 & 89.76 & 71.82 & 103.18 & 0.25274 & 7.82 & -16.58 & 33.66 & 0.37863 \\
\hline 9.35 & 5.78 & 2.44 & 1.18 & 3.60 & 0.01601 & 89.91 & 69.76 & 107.79 & 0.25384 & 0.39 & -26.18 & 33.83 & 0.36439 \\
\hline 9.35 & 6.55 & 2.74 & 1.69 & 3.74 & 0.01405 & 86.15 & 69.63 & 112.27 & 0.25837 & 5.04 & -15.34 & 22.68 & 0.28641 \\
\hline 9.35 & 7.33 & 2.73 & 1.60 & 4.00 & 0.01174 & 85.17 & 69.80 & 101.13 & 0.17393 & 5.90 & -15.49 & 25.62 & 0.22425 \\
\hline 9.35 & 12.67 & 2.84 & 1.72 & 4.21 & 0.00 & 88.01 & 72.13 & 102.69 & 0.07623 & 8.57 & -11.90 & 30.41 & 0.11093 \\
\hline 9.35 & .45 & 2.83 & 1.95 & 3.84 & 0.0 & 87.13 & 72.54 & 98.77 & 0.18044 & 9.54 & -9.93 & 25.76 & .25508 \\
\hline 9.35 & 14.22 & 2.94 & 2.08 & 3.88 & 0.01297 & 86.91 & 75.98 & 98.31 & 0.17069 & 12.18 & -9.37 & 32.52 & 0.28306 \\
\hline 9.35 & 15.00 & 2.52 & 1.54 & 3.43 & 0.01351 & 84.81 & 71.92 & 97.94 & 0.19655 & 13.46 & -9.71 & 42.53 & 0.33060 \\
\hline 9.35 & .78 & 2.55 & 1.61 & 3.51 & & 84.60 & 68.50 & 101.53 & 055 & 10.82 & -15.64 & 37.44 & .31519 \\
\hline 9.35 & 16.55 & 2.90 & 1.61 & 4.00 & 0.0 & 79.00 & 63.87 & 98.82 & 0.24558 & 15.57 & -3.31 & 42.62 & 0.28751 \\
\hline 9.35 & 17.33 & 2.94 & 1.65 & 4.23 & 0.0 & 75.64 & 59.69 & 92.17 & 0.17604 & 16.02 & -7.74 & 36.16 & .23043 \\
\hline 9.38 & 2.67 & 2.57 & 1.48 & 3.63 & & 80.28 & 65.06 & 94.43 & 0.22071 & -1.52 & -20.99 & 27.97 & .35623 \\
\hline 9.38 & 12.67 & 2.72 & 1.90 & 3.74 & 0.0 & 84.72 & 70.51 & 96.32 & 0.17156 & 8.13 & -20.64 & 27.14 & 0.27805 \\
\hline 9.44 & 7.33 & 2.79 & 1.88 & 3.67 & 0.0 & 83.92 & 69.39 & 95.62 & 0.34601 & 3.95 & -14.16 & 23.40 & .53605 \\
\hline 9.44 & 7.33 & 2.72 & 1.91 & 3.57 & & 77.53 & 62.92 & 55 & 0.40958 & 14.56 & 0.15 & 36.53 & .53551 \\
\hline 9.52 & 7.33 & 2.49 & 1.71 & 3.28 & 0.0 & 82.94 & 77.10 & 88.38 & 0.34194 & 4.92 & -13.23 & 21.47 & 0.88274 \\
\hline 9.52 & 33 & 2.79 & .95 & 3.52 & 24 & 71.71 & 58.78 & 82.05 & 0.60254 & 16.00 & -1.24 & 33.12 & .97676 \\
\hline 9.60 & 7.33 & 2.68 & 1.87 & 3.53 & 19 & 80.00 & 64.86 & 92.14 & 0.43754 & 5.17 & -12.68 & 21.26 & .52562 \\
\hline 9.60 & 17.33 & 2.84 & 1.89 & 3.67 & 0.0 & 71.18 & 56.43 & 83.71 & 0.41890 & 15.70 & -2.30 & 30.30 & 0.49210 \\
\hline 9.69 & 33 & 2.41 & 1.62 & 3.10 & & 82.25 & 71.84 & 89.68 & 075 & 3.82 & -14.07 & 25.13 & .01124 \\
\hline 9.69 & 17.33 & 2.73 & 2.05 & 3.71 & 01 & 72.34 & 61.96 & 82.78 & 0.52134 & 17.57 & -0.28 & 37.26 & 0.91096 \\
\hline 9.77 & 7.33 & 2.62 & 1.57 & 3.67 & 0.0 & 80.85 & 67.14 & 96.01 & 0.41971 & 6.71 & -8.64 & 27.25 & 0.55459 \\
\hline 77 & 33 & 2.83 & 74 & 3.98 & & 71.15 & 58.48 & 34 & 237 & 8.05 & 0.78 & 39.21 & .47502 \\
\hline 9.84 & 2.67 & 2.53 & 1.32 & 3.66 & 05 & 79.57 & 63.30 & 95.39 & 0.20850 & -0.73 & -28.31 & 19.46 & 0.34758 \\
\hline 9.84 & 12.67 & 2.74 & 1.76 & 3.79 & & 83.96 & 71.54 & 96.46 & 048 & 8.20 & -13.28 & 29.31 & .28066 \\
\hline 9.85 & 67 & 2.52 & 1.65 & 3.63 & & 75.76 & 59.83 & 91.36 & 020 & 3.35 & -23.18 & 22.07 & .15831 \\
\hline 9.85 & 3.45 & 2.46 & 1.53 & 3.52 & & 78.55 & 62.45 & 94.59 & 949 & -1.84 & -23.66 & 22.87 & .34968 \\
\hline 9.85 & 4.22 & 2.34 & 1.48 & 3.51 & & 83.15 & 67.94 & 97.47 & 0.22785 & 2.59 & -23.20 & 27.45 & 0.36714 \\
\hline 9.85 & 00 & 2.18 & 0.90 & 3.07 & & 85.02 & 68.23 & 00.69 & 24943 & 5.34 & -29.55 & 29.02 & .40516 \\
\hline 9.85 & 5.78 & 2.50 & 1.49 & 3.55 & & 85.92 & 73.01 & 103.15 & 377 & 0.23 & -22.42 & 21.04 & 0.38149 \\
\hline 9.85 & 6.55 & 2.58 & 1.65 & 3.64 & & 82.14 & 60.98 & 99.25 & 547 & 4.83 & -11.57 & 32.70 & .30561 \\
\hline 9.85 & 7.33 & 2.57 & 1.64 & 3.73 & & 80.60 & 61.00 & 2.75 & 628 & 4.36 & -17.95 & 30.00 & .26300 \\
\hline 9.85 & 67 & 2.66 & & 3.61 & & 84.70 & 71.23 & & 657 & 9.65 & -10.13 & 28.62 & .11923 \\
\hline 9.85 & .45 & 2.63 & 1.82 & 3.58 & & 83.72 & 71.81 & 96.21 & 0.20024 & 0.05 & -12.81 & 36.19 & .30673 \\
\hline 9.85 & .22 & 2.64 & 1.67 & 3.71 & 44 & 83.81 & 69.01 & 100.96 & 0.20553 & 5.64 & -6.54 & 36.24 & .29047 \\
\hline 9.85 & .00 & 2.41 & 1.60 & 3.57 & & 81.07 & 64.81 & 96.71 & 970 & 4.34 & -11.56 & 39.27 & .34684 \\
\hline 9.85 & .78 & 2.58 & 1.72 & 3.64 & & 78.32 & 63.03 & 89.93 & 0.20649 & 3.85 & -11.22 & 38.78 & .35918 \\
\hline 9.85 & .55 & 2.69 & 1.67 & 3.88 & & 73.44 & 56.20 & 91.47 & 0.25076 & 6.37 & -5.73 & 40.75 & 0.32455 \\
\hline 9.85 & 17.33 & 2.69 & 1.37 & 4.05 & & 72.46 & 53.70 & 84 & 974 & 15.09 & -8.53 & 39.41 & 0.25125 \\
\hline 9.93 & 2.67 & 2.56 & 1.74 & 3.67 & & 77.31 & 62.79 & 91.88 & 0.41053 & -3.14 & -21.16 & 16.96 & 0.54535 \\
\hline 9.93 & 12.67 & 2.54 & 1.83 & 3.39 & & 84.70 & 68.58 & 93.45 & 0.28173 & 9.41 & -14.12 & 27.94 & 0.48121 \\
\hline .00 & 2.67 & 2.73 & 2.03 & 3.29 & & 72.93 & 57.96 & 84.98 & 0.74146 & -4.45 & -20.03 & 6.35 & 0.64732 \\
\hline 10.00 & 12.67 & 2.92 & 2.19 & 3.83 & & 76.45 & 65.55 & 86.77 & 0.61705 & 9.09 & -3.07 & 27.61 & 0.64350 \\
\hline 10.07 & 2.67 & 2.95 & 1.83 & 4.09 & 393 & 71.66 & 63.25 & 83.97 & 0.35496 & -4.26 & -22.37 & 10.14 & 0.52648 \\
\hline 10.07 & .67 & 2.85 & 2.17 & 3.79 & 07 & 78.23 & 64.97 & 86.16 & 095 & 9.26 & -10.35 & 25.64 & 0.48862 \\
\hline 10.13 & 2.67 & 3.01 & 2.39 & 4.01 & 0.04778 & 70.59 & 62.59 & 78.69 & 0.54953 & -4.79 & -24.45 & 11.18 & 1.13787 \\
\hline 10.13 & 12.67 & 2.80 & 2.19 & 3.57 & 0.04006 & 77.99 & 70.92 & 86.04 & 0.43030 & 7.79 & -8.28 & 23.29 & 0.76011 \\
\hline 10.20 & 2.67 & 3.02 & 2.19 & 4.02 & & 70.59 & 60.50 & 82.15 & 0.36069 & -2.10 & -22.12 & 14.78 & 0.54177 \\
\hline 10.20 & 12.67 & 2.85 & 1.00 & 3.79 & 0.02839 & 75.34 & 64.59 & 84.06 & 0.29039 & 10.50 & $\begin{array}{l}-11.17 \\
\end{array}$ & 26.50 & 0.51479 \\
\hline 10.27 & 2.67 & 2.96 & 1.85 & 4.08 & 0.00647 & 70.89 & 53.93 & 86.04 & 0.07526 & -2.76 & -24.29 & 26.80 & 0.11730 \\
\hline
\end{tabular}




\begin{tabular}{|c|c|c|c|c|c|c|c|c|c|c|c|c|c|}
\hline 10.27 & 3.45 & 2.84 & 1.88 & 3.88 & 0.01496 & 72.31 & 58.56 & 85.26 & 0.18895 & -1.52 & -20.72 & 16.82 & 0.27627 \\
\hline 10.27 & 4.22 & 2.76 & 1.74 & 4.07 & 0.01689 & 77.81 & 61.58 & 93.02 & 0.21084 & 3.99 & -23.99 & 29.99 & 0.33086 \\
\hline 0.27 & 00 & 2.31 & .40 & 3.35 & 01297 & 78.83 & 55.15 & 93.16 & .21053 & 4.80 & -20.29 & 34.63 & .30554 \\
\hline 10.27 & 78 & 2.45 & .66 & 3.55 & 0.01453 & 78.24 & 61.79 & 91.53 & 0.20573 & -0.80 & -19.05 & 23.39 & .32388 \\
\hline 10.27 & 6.55 & 2.82 & 1.88 & 3.89 & 0.01397 & 77.27 & 57.12 & 92.61 & 0.20457 & 1.57 & -17.65 & 20.89 & 0.27367 \\
\hline 10.27 & 7.33 & 2.90 & .96 & 3.99 & .01300 & 75.24 & 61.80 & 87.91 & .15956 & 4.18 & -15.52 & 24.63 & .24223 \\
\hline 10.27 & 12.67 & 2.94 & 2.02 & 4.06 & 0.00518 & 77.11 & 65.71 & 88.88 & 0.06284 & 9.59 & -12.38 & 29.72 & 0.09768 \\
\hline 10.27 & 3.45 & 2.95 & 2.03 & 3.91 & 0.01267 & 76.97 & 63.32 & 89.93 & 0.15355 & 9.79 & -10.34 & 28.48 & 0.24100 \\
\hline 10.27 & 22 & 2.80 & 1.91 & 3.81 & 0.01505 & 76.71 & 61.74 & 91.01 & 0.19743 & 14.20 & -2.45 & 36.30 & 0.27499 \\
\hline 10.27 & .00 & 2.62 & 1.48 & 3.61 & 0.01263 & 74.63 & 57.01 & 88.64 & 0.17789 & 7.82 & -12.70 & 28.13 & 0.26011 \\
\hline 10.27 & 15.78 & 2.87 & 1.92 & 3.83 & 0.01432 & 73.20 & 55.30 & 88.27 & 0.20071 & 7.95 & -14.99 & 27.72 & 0.28168 \\
\hline 10.27 & .55 & 2.99 & 1.84 & 4.21 & 0.01 & 69.90 & 55.83 & 81.35 & 236 & 11.83 & -7.09 & 31.01 & 0.25934 \\
\hline 10.27 & 17.33 & 2.99 & 1.76 & 4.19 & 0.01295 & 66.92 & 48.24 & 83.83 & 0.17668 & 12.81 & -8.27 & 34.13 & 0.23356 \\
\hline 10.29 & 2.67 & 2.91 & 1.56 & 4.37 & 0.0 & 75.27 & 62.68 & 93.42 & & -0.76 & -18.56 & 28.97 & 0.29604 \\
\hline 10.29 & 2.67 & 2.99 & 1.97 & 4.13 & 0.0 & 79.44 & 66.66 & 95.21 & & 8.06 & -10.45 & 27.23 & 0.26508 \\
\hline 10.75 & 2.67 & 3.56 & 2.45 & 4.68 & 0.01811 & 73.58 & 62.45 & 88.42 & 993 & -2.01 & -17.82 & 12.44 & 0.27295 \\
\hline 10.75 & 12.67 & 3.48 & 2.35 & 4.69 & 0.01 & 76.97 & 67.49 & 89.57 & 069 & 8.47 & -11.23 & 20.68 & 0.24041 \\
\hline 10.86 & .67 & 3.35 & 2.11 & 4.51 & 0.0 & 71.91 & 58.21 & 83.93 & & -1.65 & -23.95 & 16.60 & 498 \\
\hline 10.86 & 3.45 & 3.31 & 2.34 & 4.37 & 0.0 & 72.41 & 58.99 & 84.27 & 90 & -1.45 & -18.44 & 16.53 & 0.27079 \\
\hline 10.86 & 4.22 & 3.20 & 2.36 & 4.24 & 0.0 & 75.39 & 62.31 & 87.61 & & 2.46 & $\begin{array}{l}-17.67 \\
\end{array}$ & 23.19 & 911 \\
\hline 10.86 & 5.00 & 2.83 & 1.95 & 4.29 & & 76.99 & 63.84 & 89.84 & & 4.94 & -14.00 & 27.82 & 386 \\
\hline 10.86 & 5.78 & 3.06 & 2.24 & 4.46 & 0.0 & 76.17 & 62.16 & 89.06 & 596 & 0.35 & -16.59 & 16.35 & 0.27171 \\
\hline 10.86 & 55 & 3.21 & 2.36 & 4.11 & 0.0 & 76.38 & 63.78 & 89.58 & & 2.95 & -13.14 & 22.13 & 38 \\
\hline 10.86 & 33 & 3.24 & 2.10 & 4.50 & & 75.37 & 61.82 & 87.67 & & 2.80 & -13.92 & 19.42 & 640 \\
\hline 10.86 & 2.67 & 3.27 & 2.23 & 4.33 & 0.0 & 76.28 & 64.88 & 87.12 & & 8.27 & -8.29 & 24.05 & 0.12551 \\
\hline 10.86 & .45 & 3.26 & 2.20 & 4.23 & 8 & 75.98 & 67.16 & 86.93 & & 8.67 & -6.28 & 23.18 & .22221 \\
\hline 10.86 & .22 & 3.31 & 2.17 & 4.37 & 3 & 75.53 & 65.93 & 88.25 & & 11.92 & -7.04 & 28.33 & 0.22701 \\
\hline 10.86 & .00 & 3.00 & 1.97 & 4.13 & 0.0 & 74.09 & 62.54 & 85.17 & 28 & 9.86 & -8.70 & 33.18 & 820 \\
\hline 10.86 & .78 & 3.43 & 2.28 & 4.53 & & 72.60 & 60.10 & 83.26 & & 9.01 & -6.42 & 24.15 & 94 \\
\hline 10.86 & 16.55 & 3.60 & 2.56 & 4.66 & 0.1 & 68.63 & 51.76 & 82.16 & 35 & 11.23 & -8.11 & 25.00 & 0.2 \\
\hline 10.86 & 17.33 & 3.58 & 2.41 & 4.88 & 0.0 & 66.93 & 49.98 & 77.30 & 89 & 11.49 & -12.75 & 26.46 & 491 \\
\hline 92 & 67 & 3.55 & 2.89 & 4.06 & & 72.16 & 63.13 & 77.83 & & -1.59 & -13.00 & 7.18 & 15 \\
\hline 10.92 & 12.67 & 3.43 & 2.54 & 4.37 & & 77.78 & 71.75 & 85.37 & & 6.40 & -11.51 & 19.02 & 320 \\
\hline 10.97 & 2.67 & 3.60 & 2.60 & 4.41 & & 71.73 & 64.99 & 80.63 & & 0.81 & -12.22 & 18.25 & 914 \\
\hline 10.97 & 67 & 3.31 & 2.68 & 3.76 & & 75.28 & 68.76 & 81.61 & & 6.36 & -2.86 & 14.13 & 540 \\
\hline 11.03 & .67 & 3.32 & 2.59 & 3.97 & & 70.41 & 62.45 & 80.75 & & -3.33 & -12.74 & 9.51 & 299 \\
\hline 11.03 & 2.67 & 3.38 & 2.65 & 4.02 & & 75.51 & 67.72 & 84.02 & & 11.10 & 0.20 & 25.90 & 132 \\
\hline 11.09 & 2.67 & 3.53 & 2.80 & 4.45 & & 69.25 & 61.03 & 80.97 & & -3.64 & -15.28 & 7.07 & 571 \\
\hline 11.09 & .67 & 3.26 & 2.63 & 3.79 & & 80.89 & 73.05 & 86.57 & & 8.50 & -6.82 & 22.48 & 268 \\
\hline 1 & 2.67 & 3.17 & 2.54 & 3.68 & & 70.90 & 62.96 & 78.64 & & -4.28 & -15.92 & 8.54 & \\
\hline 11.14 & 67 & 3.22 & 2.67 & 3.60 & & 78.85 & 68.98 & 85.59 & 45 & 8.65 & -1.22 & 18.81 & 355 \\
\hline 11.20 & 2.67 & 3.34 & 2.20 & 4.44 & & 72.64 & 60.03 & 86.78 & & -2.57 & -21.67 & 16.68 & 006 \\
\hline 11.20 & 3.45 & 3.31 & 2.21 & 4.31 & & 72.63 & 60.50 & 85.67 & & -0.52 & -17.94 & 18.93 & 124 \\
\hline 11.20 & 4.22 & 3.35 & 2.45 & 4.26 & 18 & 73.72 & 62.84 & 84.27 & 17 & -0.44 & -17.75 & 14.92 & .25269 \\
\hline 11.20 & 5.00 & 3.07 & 2.10 & 4.14 & & 78.71 & 65.93 & 89.15 & & 3.18 & -12.91 & 22.82 & 0.24708 \\
\hline 11.20 & 5.78 & 2.77 & 1.94 & 4.00 & & 78.75 & 63.89 & 89.91 & & -2.32 & -21.69 & 15.00 & 420 \\
\hline 11.20 & 6.55 & 3.22 & 2.10 & 4.29 & & 76.78 & 61.32 & 87.99 & 160 & -1.12 & -19.70 & 17.88 & 0.24231 \\
\hline 11.20 & .33 & 3.11 & 2.19 & 4.00 & & 76.47 & 64.70 & 87.18 & & 2.84 & -11.75 & 17.71 & 450 \\
\hline 11.20 & 2.67 & 3.37 & 2.31 & 4.62 & & 77.12 & 65.41 & 87.69 & & 8.18 & $\begin{array}{l}-13.69 \\
\end{array}$ & 25.68 & 985 \\
\hline 11.20 & 3.45 & 3.39 & 2.24 & 4.36 & & 77.13 & 66.83 & 87.86 & & 7.97 & -6.79 & 23.69 & 599 \\
\hline 11.20 & 22 & 3.31 & 2.50 & 4.31 & & 77.73 & 67.48 & 89.85 & 503 & 8.53 & -6.54 & 23.17 & 0.22446 \\
\hline 11.20 & .00 & 3.00 & 2.11 & 4.27 & & 75.11 & 61.47 & 85.61 & 889 & 11.50 & -14.74 & 32.31 & 0.25028 \\
\hline 11.20 & .78 & 3.19 & 2.11 & 4.21 & & 74.63 & 62.04 & 86.63 & 477 & 4.38 & -16.93 & 25.54 & 0.25762 \\
\hline 11.20 & .55 & 3.54 & 2.42 & 4.51 & & 71.49 & 61.30 & 83.60 & & 9.86 & -7.78 & 21.97 & 323 \\
\hline 11.20 & 17.33 & 3.51 & 2.55 & 4.70 & 0.01267 & 70.08 & 57.29 & 80.71 & 0.14058 & 11.04 & -6.87 & 26.03 & 0.19210 \\
\hline 11.21 & 2.67 & 3.53 & 2.17 & 4.77 & 0.01728 & 73.26 & 63.27 & 85.50 & 0.17179 & -2.00 & $\begin{array}{l}-16.07 \\
\end{array}$ & 11.06 & 0.24033 \\
\hline 11.21 & 12.67 & 3.41 & 2.37 & 4.38 & & 77.05 & 66.69 & 88.63 & & 6.92 & -7.31 & 22.73 & 0.24655 \\
\hline 11.28 & 1.00 & 3.11 & 2.65 & 3.62 & 0.03808 & 76.03 & 67.99 & 82.81 & 0.65044 & 2.61 & -6.42 & 17.01 & 1.03725 \\
\hline 11.28 & 17.33 & 3.33 & 2.68 & 3.78 & 0.03724 & 68.86 & 62.43 & 74.61 & 0.47508 & 8.58 & 0.94 & 18.05 & 0.60385 \\
\hline
\end{tabular}




\begin{tabular}{|c|c|c|c|c|c|c|c|c|c|c|c|c|c|}
\hline 11.37 & 7.33 & 3.36 & 2.67 & 4.03 & 0.07151 & 76.18 & 68.85 & 80.62 & 0.57240 & 2.88 & -9.19 & \begin{tabular}{|l|}
17.21 \\
\end{tabular} & 1.09148 \\
\hline 11.37 & 17.33 & 3.77 & 3.17 & 4.37 & 0.04765 & 69.71 & 60.46 & 77.66 & 0.53625 & 11.61 & 3.75 & 20.50 & 0.69196 \\
\hline 11.45 & 33 & 3.18 & 2.69 & 3.68 & 03468 & 75.58 & 9.32 & 83.49 & 0.60160 & 3.25 & 10.58 & \begin{tabular}{|l|}
16.51 \\
\end{tabular} & .11368 \\
\hline .45 & .33 & 3.55 & 2.93 & 4.15 & 0.04564 & 70.30 & 59.93 & 75.31 & 0.60307 & 11.02 & -2.10 & 20.72 & .78545 \\
\hline 11.53 & 7.33 & 3.19 & 2.48 & 3.94 & 0.05648 & 78.28 & 64.97 & 85.66 & 0.83348 & 5.34 & -5.60 & 12.42 & .59776 \\
\hline 11.53 & .33 & 3.35 & 2.70 & 4.06 & 0.05256 & 71.13 & 65.94 & 83.10 & .54501 & 11.80 & 3.23 & 20.96 & .68796 \\
\hline 11.62 & 7.33 & 3.00 & 2.52 & 3.62 & 0.05133 & 78.58 & 67.05 & 85.45 & 0.98692 & -2.09 & -10.33 & 13.66 & 0.96505 \\
\hline 11.62 & 17.33 & 3.28 & 2.62 & 3.78 & 0.05860 & 72.75 & 64.15 & 83.29 & 0.82377 & 10.82 & -6.53 & 19.01 & 0.94697 \\
\hline 11.67 & 2.67 & 3.40 & 2.31 & 4.43 & 0.00415 & 73.61 & 60.78 & 85.58 & 0.04451 & -1.31 & -18.27 & 17.60 & 0.06459 \\
\hline 11.67 & 3.45 & 3.41 & 2.38 & 4.40 & 0.01220 & 75.62 & 64.21 & 88.08 & 0.14183 & -0.84 & -18.72 & 19.97 & 0.18412 \\
\hline 11.67 & 4.22 & 3.27 & 2.23 & 4.57 & 0.01457 & 78.52 & 63.80 & 89.79 & 0.13708 & 2.49 & -17.16 & 25.18 & 0.20874 \\
\hline 11.67 & 5.00 & 2.77 & 1.83 & 4.48 & 0.01 & 78.45 & 64.14 & 89.61 & 0.16323 & 1.65 & -20.82 & 25.60 & 828 \\
\hline 11.67 & 5.78 & 3.09 & 2.04 & 4.32 & 0.01241 & 77.79 & 63.90 & 89.25 & 0.14676 & -0.59 & -19.89 & 17.48 & 0.21126 \\
\hline 11.67 & 6.55 & 3.27 & 2.22 & 4.62 & 0.01 & 75.60 & 63.03 & 88.92 & 0.16100 & 2.65 & -18.77 & 19.71 & 144 \\
\hline & 7.33 & 3.30 & 2.26 & 4.52 & 0.0 & 75.02 & 61.65 & 87.25 & 0.04773 & 4.10 & -14.58 & 23.37 & 521 \\
\hline 11.67 & 2.67 & 3.36 & 2.26 & 4.71 & 0.00387 & 76.28 & 62.66 & 87.55 & 0.04224 & 7.64 & -10.83 & 25.38 & 0.06205 \\
\hline 11.67 & .45 & 3.42 & 2.20 & 4.57 & 0.01 & 75.07 & 64.07 & 84.72 & 0.11631 & 9.70 & -10.17 & 25.12 & 076 \\
\hline & 22 & 3.28 & 2.13 & 4.41 & & 74.12 & 61.92 & 85.07 & 795 & 12.36 & -4.36 & 27.39 & \\
\hline 11.67 & .00 & 3.13 & 2.04 & 4.12 & 0.0 & 74.03 & 61.32 & 86.37 & 0.12730 & 6.20 & -9.83 & 26.88 & 527 \\
\hline 11.67 & .78 & 3.46 & 2.35 & 4.68 & 0.0 & 73.83 & 57.99 & 84.41 & 0.12881 & 8.93 & -6.72 & 27.50 & \\
\hline & .55 & 3.55 & 2.21 & 4.55 & & 70.50 & 58.20 & 83.11 & 0.14326 & 12.54 & -2.66 & 27.48 & \\
\hline 11.67 & .33 & 3.54 & 2.16 & 4.68 & 0.0 & 69.39 & 55.87 & 82.92 & 0.04549 & 12.34 & -12.52 & 31.75 & 345 \\
\hline 0 & 2.67 & 3.38 & 2.32 & 4.51 & 0.0 & 72.15 & 61.60 & 83.78 & 158 & -1.16 & -15.00 & 16.34 & \\
\hline & 45 & 3.51 & 2.28 & 4.72 & & 74.09 & 64.89 & 87.86 & 575 & -0.89 & -17.85 & 16.35 & \\
\hline 11.70 & 4.22 & 3.36 & 2.29 & 4.54 & 0.0 & 76.66 & 64.10 & 87.01 & 557 & 1.67 & -12.70 & 16.28 & 476 \\
\hline & .00 & 2.82 & 1.93 & 4.06 & 0 & 78.38 & 62.75 & 91.25 & 110 & 4.16 & -17.20 & 26.96 & \\
\hline & 78 & 2.99 & 1.93 & 3.99 & & 78.29 & 64.80 & 88.50 & 586 & -0.28 & -20.18 & 17.09 & \\
\hline 11.70 & 6.55 & 3.19 & 2.27 & 4.19 & 0.0 & 76.29 & 65.80 & 87.05 & 005 & 2.42 & -16.19 & 19.28 & \\
\hline & 33 & 3.09 & 2.01 & 4.14 & & 76.15 & 64.33 & 88.88 & & 2.01 & -19.06 & \begin{tabular}{|l|}
21.06 \\
\end{tabular} & \\
\hline 70 & 67 & 3.37 & 2.37 & 4.53 & & 76.06 & 65.01 & 85.53 & 240 & 7.39 & -16.16 & 24.18 & 443 \\
\hline 11.70 & .45 & 3.59 & 2.64 & 4.93 & 0.0 & 75.62 & 62.61 & 87.29 & 0.17710 & 8.63 & -5.31 & 25.69 & 396 \\
\hline & 22 & 3.29 & 2.26 & 4.20 & & 75.85 & 64.31 & 85.03 & 957 & 13.12 & -3.74 & 29.09 & \\
\hline 70 & .00 & 2.92 & 1.96 & 3.76 & 0 & 73.48 & 59.63 & 83.51 & 0.17787 & 8.02 & -10.44 & \begin{tabular}{|l|}
27.19 \\
\end{tabular} & 005 \\
\hline & 78 & 3.38 & 2.45 & 4.50 & & 73.08 & 63.23 & 82.94 & 976 & 8.48 & -6.73 & 24.66 & 26 \\
\hline & 55 & 3.43 & 2.31 & 4.38 & & 69.43 & 56.41 & 79.01 & 225 & 2.06 & -6.04 & \begin{tabular}{|l|}
27.28 \\
\end{tabular} & \\
\hline & .33 & 3.42 & 2.19 & 4.52 & & 69.77 & 56.03 & 84.22 & 481 & 11.64 & -5.87 & 27.26 & 044 \\
\hline 12.09 & 7.33 & 3.34 & 2.38 & 4.74 & 0.0 & 73.05 & 64.45 & 83.97 & 0.22708 & 4.13 & -9.11 & 20.23 & 528 \\
\hline 12.09 & .33 & 3.53 & 2.52 & 4.83 & & 66.95 & 54.26 & 77.27 & 0.21148 & 11.97 & 12.34 & 27.94 & 175 \\
\hline 12.50 & 7.33 & 3.35 & 2.52 & 4.25 & & 73.57 & 64.07 & 83.88 & 4777 & 1.93 & -11.25 & 18.83 & 586 \\
\hline 12.50 & .33 & 3.65 & 2.66 & 4.72 & & 67.23 & 57.78 & 78.50 & 925 & 12.71 & -5.62 & 28.19 & \\
\hline 12.92 & .33 & 3.38 & 2.57 & 4.20 & & 73.31 & 61.60 & 84.74 & 752 & 4.35 & -7.78 & \begin{tabular}{|l|}
19.11 \\
\end{tabular} & 93 \\
\hline 12.92 & .33 & 3.78 & 2.82 & 4.64 & & 66.89 & 56.30 & 76.12 & 520 & 13.03 & -1.32 & 26.21 & 349 \\
\hline 13.34 & 7.33 & 3.39 & 2.61 & 4.40 & & 73.80 & 63.20 & 82.34 & 0.23523 & 3.22 & -10.55 & \begin{tabular}{|l|}
16.19 \\
\end{tabular} & 621 \\
\hline 13.34 & .33 & 3.71 & 2.85 & 4.51 & & 66.74 & 57.95 & 75.40 & 0.21436 & 11.59 & -1.62 & 26.44 & 038 \\
\hline 13.75 & 7.33 & 3.23 & 2.16 & 4.30 & & 73.37 & 58.42 & 84.98 & 751 & 3.30 & -11.58 & 21.97 & 603 \\
\hline 13.75 & .33 & 3.53 & 2.60 & 4.54 & & 67.31 & 58.53 & 79.41 & 848 & 11.68 & -6.57 & \begin{tabular}{|l|}
26.80 \\
\end{tabular} & 790 \\
\hline 14.22 & 2.67 & 3.17 & 2.02 & 4.39 & & 70.65 & 56.53 & 84.76 & 0.04559 & 0.00 & -19.74 & 19.41 & 647 \\
\hline 14.22 & 3.45 & 2.84 & 2.08 & 3.89 & & 74.67 & 60.23 & 85.28 & 779 & 4.13 & -13.82 & 24.02 & 368 \\
\hline 14.22 & 4.22 & 2.54 & 1.60 & 3.68 & & 72.80 & 57.63 & 82.98 & 0.21941 & 0.72 & -20.23 & \begin{tabular}{|l|}
22.34 \\
\end{tabular} & 0.33585 \\
\hline 14.22 & 5.00 & 2.91 & 1.91 & 4.10 & & 73.41 & 61.90 & 85.56 & 0.17155 & -3.38 & -21.56 & 18.24 & 0.29682 \\
\hline 14.22 & 5.78 & 3.21 & 2.17 & 4.35 & & 73.67 & 63.38 & 85.01 & 510 & 0.46 & -16.11 & 15.60 & 649 \\
\hline 14.22 & 6.55 & 3.23 & 2.06 & 4.19 & & 71.98 & 61.02 & 84.41 & & 2.49 & -18.36 & \begin{tabular}{|l|}
16.76 \\
\end{tabular} & 0.24630 \\
\hline 14.22 & 7.33 & 3.23 & 2.09 & 4.31 & & 71.84 & 61.03 & 85.52 & 0.04253 & 3.42 & $\begin{array}{l}-14.77 \\
\end{array}$ & 31.64 & 0.06430 \\
\hline 14.22 & 67 & 3.21 & 2.11 & 4.30 & & 72.20 & 61.76 & 86.24 & 355 & 6.55 & -13.05 & 25.84 & 946 \\
\hline 14.22 & 13.45 & 2.93 & 2.01 & 3.71 & 0.01309 & 67.95 & 56.73 & 78.24 & 0.14385 & 11.10 & -4.69 & 31.92 & 0.26664 \\
\hline 14.22 & 14.22 & 2.98 & 2.11 & 4.09 & 0.01507 & 68.20 & 52.49 & 77.36 & 0.15027 & 5.83 & -10.29 & 29.69 & 0.27106 \\
\hline 14.22 & 00 & 3.19 & 2.23 & 4.43 & 20 & 69.28 & 54.54 & 80.21 & 0.17249 & 7.04 & -9.80 & 26.42 & 326 \\
\hline 14.22 & 15.78 & 3.38 & 2.39 & 4.46 & 0.01577 & 67.93 & 58.09 & 80.07 & 0.16814 & 10.40 & -6.80 & \begin{tabular}{|l|}
27.31 \\
\end{tabular} & 0.25848 \\
\hline 14.22 & 16.55 & 3.32 & 2.01 & 4.24 & 0.01639 & 66.04 & 54.96 & 77.33 & 0.15272 & 10.54 & -8.14 & 30.55 & 0.28350 \\
\hline
\end{tabular}




\begin{tabular}{|c|c|c|c|c|c|c|c|c|c|c|c|c|c|}
\hline 14.22 & 17.33 & 3.37 & 2.26 & 4.39 & 0.00390 & 65.03 & 52.99 & 80.85 & 0.03883 & 11.95 & $\begin{array}{l}-10.39 \\
\end{array}$ & 33.17 & 0.06861 \\
\hline 14.68 & 2.67 & 3.61 & 2.45 & 4.62 & 0.01527 & 65.81 & 50.54 & 77.25 & 0.16033 & -0.25 & -18.37 & 18.51 & 0.21399 \\
\hline 14.68 & 12.67 & 3.71 & 2.80 & 4.73 & 0.01289 & 65.65 & 54.95 & 75.89 & 0.13389 & 4.99 & -7.33 & 19.36 & .19113 \\
\hline 15.15 & 2.67 & 3.67 & 2.81 & 4.72 & 0.01368 & 65.56 & 53.93 & 75.57 & 0.14396 & -0.38 & $\begin{array}{l}-16.07 \\
\end{array}$ & 14.72 & .21282 \\
\hline 15.15 & 12.67 & 3.70 & 2.91 & 4.63 & 0.01266 & 65.90 & 55.75 & 74.32 & 0.12353 & 4.08 & -11.31 & 16.89 & 0.19769 \\
\hline 15.61 & 2.67 & 3.70 & 2.70 & 4.60 & 0.01463 & 65.97 & 54.98 & 74.74 & 0.15642 & -0.65 & -18.04 & 14.45 & 0.23569 \\
\hline 15.61 & 12.67 & 3.74 & 2.88 & 4.68 & 0.01305 & 65.96 & 55.44 & 74.43 & 0.13620 & 4.92 & -8.05 & 18.75 & 0.21120 \\
\hline 16.07 & 2.67 & 3.67 & 2.72 & 4.59 & 0.01335 & 66.64 & 52.63 & 77.39 & 0.16108 & -0.35 & -15.62 & 14.25 & 0.21443 \\
\hline 16.07 & 12.67 & 3.76 & 3.01 & 4.70 & 0.01180 & 67.17 & 58.78 & 75.03 & 850 & 3.90 & -7.99 & 16.03 & 0.17008 \\
\hline 16.54 & 2.67 & 3.67 & 2.72 & 4.66 & 0.01444 & 66.76 & 56.70 & 76.74 & 0.13135 & 0.00 & -12.80 & 16.45 & 0.20113 \\
\hline 16.54 & 12.67 & 3.66 & 2.91 & 4.48 & 0.01212 & 66.88 & 55.88 & 74.97 & 0.11779 & 4.83 & -9.60 & 19.16 & 0.19111 \\
\hline 16.99 & 2.67 & 3.48 & 2.46 & 4.52 & 0.00381 & 67.00 & 54.95 & 78.21 & 931 & 0.02 & -17.72 & 16.05 & 693 \\
\hline 16.99 & 3.45 & 3.52 & 2.70 & 4.84 & 0.01113 & 66.65 & 52.48 & 78.93 & 989 & 0.59 & -14.29 & 16.88 & 0.17471 \\
\hline 16.99 & 4.22 & 3.44 & 2.50 & 4.43 & 0.0 & 68.40 & 58.76 & 77.21 & & 3.18 & -11.41 & 17.94 & \\
\hline 16.99 & 5.00 & 3.17 & 2.35 & 4.28 & 0.0 & 66.55 & 52.44 & 77.22 & & 4.21 & -12.91 & 21.97 & \\
\hline 16.99 & 5.78 & 3.40 & 2.34 & 4.54 & 0.01290 & 66.33 & 52.90 & 75.12 & 704 & 0.22 & -16.84 & 16.71 & 514 \\
\hline 16.99 & 6.55 & 3.57 & 2.55 & 4.57 & 0.0 & 66.72 & 56.09 & 75.39 & 52 & 2.92 & -12.95 & 20.21 & 057 \\
\hline 16.99 & 7.33 & 3.61 & 2.66 & 4.56 & & 65.90 & 53.12 & 75.77 & & 3.52 & -10.18 & 21.18 & \\
\hline 16.99 & 12.67 & 3.51 & 2.65 & 4.44 & 0.0 & 66.56 & 53.17 & 76.32 & 204 & 4.37 & -18.19 & 23.67 & 090 \\
\hline 16.99 & 13.45 & 3.61 & 2.79 & 4.75 & 0.0 & 66.70 & 55.76 & 76.22 & & 5.58 & $\begin{array}{l}-12.44 \\
\end{array}$ & 28.93 & \\
\hline 16.99 & 4.22 & 3.52 & 2.50 & 4.63 & & 65.07 & 53.77 & 73.80 & & 10.41 & -5.82 & 26.02 & \\
\hline 16.99 & 15.00 & 3.31 & 2.38 & 4.21 & 0.0 & 62.57 & 50.50 & 71.31 & & 6.25 & -11.58 & 30.50 & 576 \\
\hline 16.99 & 15.78 & 3.50 & 2.67 & 4.36 & 0.0 & 64.08 & 50.59 & 73.12 & & 5.73 & -11.96 & 23.70 & \\
\hline 16.99 & .55 & 3.53 & 2.57 & 4.61 & & 62.65 & 50.51 & 71.19 & & 8.35 & -6.49 & 27.14 & \\
\hline 16.99 & 17.33 & 3.57 & 2.54 & 4.58 & 0.0 & 60.97 & 46.66 & 71.86 & 90 & 8.54 & -9.24 & 28.50 & 666 \\
\hline 17.41 & 7.33 & 3.57 & 2.65 & 4.33 & 2 & 66.35 & 56.74 & 76.30 & & 3.31 & -11.21 & 21.61 & \\
\hline 17.41 & 17.33 & 3.59 & 2.77 & 4.62 & 0.0 & 61.08 & 49.48 & 73.34 & & 9.04 & -4.04 & 22.32 & \\
\hline 17.83 & 7.33 & 3.70 & 3.04 & 4.40 & 0.0 & 63.89 & 53.75 & 69.87 & 78 & 3.04 & -8.50 & 14.47 & 943 \\
\hline 17.83 & 17.33 & 3.60 & 2.79 & 4.39 & & 59.25 & 47.26 & 68.43 & & 8.95 & -8.40 & 24.78 & \\
\hline 18.24 & 7.33 & 3.68 & 2.85 & 4.46 & 0.0 & 64.60 & 51.93 & 72.58 & & 2.95 & -11.28 & 13.20 & 698 \\
\hline 18.24 & 17.33 & 3.80 & 3.22 & 4.65 & 0.0 & 60.28 & 46.33 & 71.99 & 165 & 9.01 & -3.80 & 20.72 & 376 \\
\hline 18.66 & 33 & 4.11 & 3.59 & 4.71 & & 65.75 & 56.15 & 75.16 & & 3.57 & -9.85 & 16.36 & \\
\hline 18.66 & 17.33 & 4.10 & 3.38 & 4.78 & 32 & 61.78 & 51.22 & 71.46 & & 9.48 & -3.50 & 20.23 & 427 \\
\hline 19.08 & 7.33 & 3.48 & 2.70 & 4.42 & & 64.31 & 54.07 & 74.04 & & 2.68 & -11.81 & 11.96 & 587 \\
\hline 19.08 & .33 & 3.63 & 2.70 & 4.62 & & 61.75 & 52.07 & 71.51 & & 9.94 & -6.34 & 24.12 & \\
\hline 19.47 & 2.67 & 3.30 & 2.29 & 4.44 & & 64.18 & 48.09 & 77.19 & & 0.88 & -16.24 & 20.65 & \\
\hline 19.47 & 3.45 & 3.29 & 2.22 & 4.31 & & 64.87 & 53.13 & 73.00 & & 0.87 & -14.66 & 21.15 & 480 \\
\hline 19.47 & 4.22 & 3.25 & 2.36 & 4.22 & & 66.98 & 56.40 & 76.65 & & 3.06 & -12.70 & 19.32 & 157 \\
\hline 19.47 & 5.00 & 3.07 & 2.12 & 4.22 & & 63.81 & 47.84 & 74.78 & & 4.80 & -15.79 & 21.85 & 084 \\
\hline 19.47 & 5.78 & 3.23 & 2.15 & 4.33 & & 63.67 & 51.58 & 73.48 & & -1.95 & -17.85 & 12.96 & \\
\hline 19.47 & .55 & 3.43 & 2.54 & 4.32 & & 64.66 & 50.89 & 77.99 & & 1.72 & -15.04 & 16.90 & 666 \\
\hline 19.47 & 7.33 & 3.43 & 2.51 & 4.43 & & 64.22 & 49.95 & 74.52 & & 2.71 & -15.18 & 20.94 & 511 \\
\hline 19.47 & 12.67 & 3.31 & 2.32 & 4.38 & & 65.39 & 54.15 & 74.94 & & 3.64 & -13.35 & 20.03 & 401 \\
\hline 19.47 & 13.45 & 3.32 & 2.51 & 4.32 & & 65.67 & 56.21 & 74.96 & & 4.66 & -9.46 & 20.52 & 494 \\
\hline 19.47 & .22 & 3.36 & 2.34 & 4.41 & & 64.66 & 52.24 & 74.03 & & 9.46 & -7.84 & 26.65 & 293 \\
\hline 19.47 & .00 & 3.13 & 2.36 & 3.91 & & 61.47 & 50.19 & 70.93 & & 5.95 & -16.08 & 24.15 & \\
\hline 19.47 & .78 & 3.37 & 2.55 & 4.28 & & 63.94 & 53.29 & 72.90 & 57 & 5.15 & -9.97 & 20.48 & 764 \\
\hline 19.47 & .55 & 3.43 & 2.57 & 4.39 & & 62.71 & 48.44 & 72.95 & & 8.21 & -8.96 & 25.33 & 811 \\
\hline 19.47 & 17.33 & 3.51 & 2.63 & 4.47 & & 60.47 & 44.38 & 71.46 & 675 & 9.01 & -10.45 & 27.29 & 568 \\
\hline 19.95 & 2.67 & 3.46 & 2.23 & 4.64 & & 56.91 & 41.94 & 74.01 & & -0.54 & -21.96 & 19.07 & 162 \\
\hline 19.95 & 12.67 & 3.52 & 2.05 & 4.54 & & 59.43 & 45.61 & 75.05 & 80 & 3.99 & -14.39 & 26.35 & 436 \\
\hline 20.42 & 2.67 & 3.88 & 3.16 & 4.70 & & 55.71 & 43.60 & 66.42 & 264 & -0.79 & $\begin{array}{l}-16.07 \\
\end{array}$ & 14.80 & 796 \\
\hline 20.42 & 12.67 & 3.89 & 3.11 & 4.62 & & 57.54 & 47.16 & 67.75 & 149 & 4.69 & -10.84 & 18.89 & 890 \\
\hline 20.90 & 2.67 & 3.88 & 3.08 & 4.52 & & 55.20 & 43.10 & 65.70 & & -0.47 & -16.58 & 16.76 & 482 \\
\hline 20.90 & 12.67 & 3.90 & 3.17 & 4.64 & 0.00696 & 57.88 & 47.90 & 67.15 & 0.11499 & 3.89 & -10.44 & 19.21 & 0.16163 \\
\hline 21.38 & 2.67 & 3.83 & 3.07 & 4.74 & & 55.54 & 42.96 & 66.48 & 0.13128 & -0.05 & -13.92 & 17.44 & 0.17674 \\
\hline 21.38 & 12.67 & 3.91 & 3.19 & 4.67 & & 57.68 & 46.32 & 67.00 & & 4.61 & -11.59 & 18.93 & 094 \\
\hline 21.85 & 2.67 & 3.89 & 2.85 & 4.78 & 0.00896 & 58.31 & 45.67 & 69.21 & 0.14315 & -0.91 & $\begin{array}{l}-15.37 \\
\end{array}$ & 16.44 & 0.16780 \\
\hline 21.85 & 12.67 & 3.92 & 3.05 & 4.74 & 0.00850 & 59.70 & 48.78 & 72.80 & 0.11503 & 4.02 & -12.91 & 18.05 & 0.16545 \\
\hline
\end{tabular}




\begin{tabular}{|c|c|c|c|c|c|c|c|c|c|c|c|c|c|}
\hline 22.37 & 2.67 & 3.69 & 2.83 & 4.68 & 0.00301 & 58.65 & 43.48 & 70.05 & 0.04560 & -0.42 & $\begin{array}{l}-17.64 \\
\end{array}$ & 18.20 & 0.06312 \\
\hline 22.37 & 3.45 & 3.71 & 2.81 & 4.42 & 0.00834 & 59.46 & 44.76 & 70.99 & 0.14017 & 0.75 & -14.87 & 17.03 & 0.18333 \\
\hline 22.37 & 4.22 & 3.74 & 2.98 & 4.54 & 00864 & 60.71 & 50.56 & 70.50 & .11370 & 4.28 & -14.01 & 21.79 & .18298 \\
\hline 22.37 & 5.00 & 3.52 & 2.71 & 4.59 & 0.00919 & 57.17 & 43.26 & 69.60 & 0.14570 & 4.19 & -15.83 & 21.62 & .22265 \\
\hline 22.37 & 5.78 & 3.72 & 2.86 & 4.57 & 0.00905 & 59.22 & 48.16 & 69.02 & 0.12561 & -0.43 & -16.38 & 15.46 & 0.18775 \\
\hline 22.37 & 6.55 & 3.82 & 2.92 & 4.57 & .00872 & 59.24 & 47.23 & 69.66 & 0.13469 & 3.09 & -14.38 & 17.30 & .17093 \\
\hline 22.37 & 7.33 & 3.79 & 2.74 & 4.58 & 0.00279 & 59.00 & 45.68 & 69.07 & 0.04458 & 2.32 & -14.17 & 20.83 & 0.05519 \\
\hline 22.37 & 12.67 & 3.76 & 2.93 & 4.49 & 0.00252 & 59.14 & 46.94 & 72.00 & 0.03720 & 3.97 & -12.56 & 21.19 & 0.05422 \\
\hline 22.37 & .45 & 3.82 & 3.09 & 4.58 & 0.00684 & 59.47 & 50.17 & 68.55 & 0.10063 & 5.37 & -11.49 & 19.28 & 0.16213 \\
\hline 22.37 & 4.22 & 3.69 & 2.94 & 4.63 & 0.00750 & 57.49 & 46.92 & 68.78 & 0.10786 & 10.07 & -5.16 & 27.49 & 0.15963 \\
\hline 22.37 & 15.00 & 3.52 & 2.75 & 4.57 & 0.00758 & 55.00 & 43.03 & 66.07 & 0.12337 & 3.78 & -11.61 & 20.47 & 0.17411 \\
\hline 22.37 & .78 & 3.70 & 3.04 & 4.61 & 0.00712 & 57.10 & 46.07 & 66.49 & 358 & 6.94 & -12.01 & 25.53 & 0.17214 \\
\hline 22.37 & 16.55 & 3.71 & 2.96 & 4.74 & 0.00753 & 54.87 & 42.44 & 66.30 & 0.13376 & 9.95 & -8.48 & 26.11 & 0.17956 \\
\hline 22.37 & 17.33 & 3.69 & 2.83 & 4.64 & 0.00 & 53.86 & 39.43 & 66.50 & 408 & 9.51 & -8.86 & 27.88 & 0.05817 \\
\hline 22.78 & 7.33 & 3.85 & 3.00 & 4.49 & 0.0 & 55.92 & 44.77 & 67.80 & 211 & 1.79 & -14.23 & 17.49 & 362 \\
\hline 22.78 & 17.33 & 3.75 & 3.10 & 4.63 & 0.00594 & 51.16 & 37.10 & 67.87 & 0.10258 & 8.62 & -10.30 & 28.46 & 0.14296 \\
\hline 23.19 & 7.33 & 3.83 & 2.95 & 4.68 & 0.00615 & 56.08 & 44.32 & 69.09 & 15 & 2.01 & -14.60 & 23.50 & 0.13739 \\
\hline 23.19 & 7.33 & 3.73 & 2.93 & 4.53 & 0.0 & 51.78 & 35.72 & 62.71 & & 8.21 & -7.66 & 25.47 & 672 \\
\hline 23.60 & 7.33 & 3.82 & 3.10 & 4.57 & 0.00621 & 56.39 & 40.21 & 66.76 & 269 & 2.04 & -12.88 & 18.93 & 159 \\
\hline 23.60 & 17.33 & 3.69 & 2.86 & 4.43 & 0.0 & 51.41 & 39.52 & 62.60 & 30 & 8.27 & -8.36 & 27.10 & \\
\hline 24.01 & .33 & 3.82 & 3.05 & 4.65 & 95 & 55.95 & 42.65 & 65.82 & 377 & 2.13 & -14.08 & 19.54 & \\
\hline 24.01 & 17.33 & 3.71 & 3.04 & 4.53 & 0.00620 & 52.06 & 37.56 & 62.95 & 090 & 8.06 & -7.69 & 26.96 & 334 \\
\hline 24.42 & 7.33 & 3.71 & 2.80 & 4.64 & 0.0 & 56.18 & 39.20 & 68.09 & 23 & 2.04 & -14.34 & 19.52 & 322 \\
\hline 24.42 & 7.33 & 3.68 & 2.90 & 4.63 & 20 & 52.32 & 35.94 & 65.96 & & 6.46 & -14.20 & 24.60 & 345 \\
\hline 24.97 & 2.67 & 3.47 & 2.60 & 4.28 & 0.00262 & 50.74 & 37.08 & 64.87 & 14 & 0.23 & -21.42 & 19.34 & 756 \\
\hline 24.97 & 3.45 & 3.50 & 2.78 & 4.30 & 55 & 52.37 & 35.23 & 64.29 & & 0.37 & -18.33 & 19.46 & 27 \\
\hline 24.97 & 4.22 & 3.52 & 2.84 & 4.48 & 65 & 54.70 & 40.06 & 66.73 & & 3.04 & -16.53 & 18.69 & \\
\hline 24.97 & 5.00 & 3.42 & 2.63 & 4.27 & 0.00877 & 52.18 & 37.19 & 65.01 & 92 & 5.37 & -19.49 & 25.24 & 308 \\
\hline 24.97 & 78 & 3.53 & 2.59 & 4.47 & 26 & 54.08 & 37.32 & 65.34 & & -2.14 & -19.66 & 21.77 & \\
\hline 24.97 & 6.55 & 3.65 & 2.92 & 4.36 & 770 & 54.95 & 40.49 & 67.67 & 56 & 1.60 & -15.80 & 20.56 & 122 \\
\hline 24.97 & 7.33 & 3.60 & 2.67 & 4.29 & 0.00271 & 54.38 & 40.35 & 66.93 & 84 & 2.30 & -14.49 & 20.84 & 655 \\
\hline 24.97 & 67 & 3.66 & 2.82 & 4.52 & 41 & 54.43 & 42.84 & 67.74 & & 3.28 & -14.08 & 18.35 & 770 \\
\hline 24.97 & .45 & 3.71 & 2.91 & 4.42 & 0.0 & 54.63 & 41.63 & 67.02 & 70 & 4.05 & -15.09 & 20.95 & 692 \\
\hline 24.97 & 22 & 3.70 & 3.12 & 4.39 & 34 & 53.18 & 41.86 & 63.28 & & 8.68 & -9.51 & 28.40 & 707 \\
\hline 24.97 & 00 & 3.50 & 2.82 & 4.31 & 43 & 51.47 & 38.80 & 66.49 & & 3.47 & -13.24 & 22.18 & 555 \\
\hline 24.97 & 78 & 3.69 & 2.64 & 4.41 & & 54.64 & 44.30 & 68.27 & & 1.42 & -15.88 & 16.01 & \\
\hline 24.97 & .55 & 3.58 & 2.88 & 4.31 & 0.00685 & 52.89 & 39.87 & 68.03 & & 5.35 & -11.16 & 26.24 & 592 \\
\hline 24.97 & 17.33 & 3.53 & 2.81 & 4.34 & 55 & 51.16 & 35.28 & 63.81 & 75 & 5.87 & -12.17 & 30.56 & 155 \\
\hline 25.42 & 2.67 & 3.48 & 2.80 & 3.97 & & 51.18 & 41.25 & 63.84 & & -0.39 & -14.95 & 18.45 & 561 \\
\hline 25.42 & 2.67 & 3.66 & 3.03 & 4.16 & & 55.91 & 46.14 & 67.57 & & 3.08 & -11.50 & 15.09 & \\
\hline 25.87 & 2.67 & 3.51 & 2.94 & 3.94 & 00 & 50.99 & 40.65 & 61.19 & 55 & -0.28 & -14.29 & 14.64 & 764 \\
\hline 25.87 & 2.67 & 3.66 & 3.11 & 4.18 & & 54.40 & 46.84 & 63.58 & & 2.45 & -9.25 & 12.87 & 968 \\
\hline 26.32 & 2.67 & 3.39 & 2.69 & 4.16 & 89 & 46.80 & 34.77 & 63.15 & & 0.71 & -17.71 & 21.38 & \\
\hline 26.32 & 2.67 & 3.55 & 2.94 & 4.40 & 83 & 51.53 & 36.51 & 67.56 & 48 & 4.98 & -11.16 & 19.73 & 158 \\
\hline 26.77 & 2.67 & 4.09 & 3.44 & 4.68 & & 47.38 & 35.60 & 57.34 & & -0.30 & -12.13 & 11.83 & 959 \\
\hline 26.77 & 12.67 & 4.22 & 3.58 & 4.99 & & 48.90 & 37.82 & 59.23 & & 3.62 & -9.04 & 14.77 & \\
\hline 27.22 & 2.67 & 4.02 & 3.52 & 4.45 & 82 & 45.47 & 37.96 & 54.19 & 322 & 0.78 & -10.56 & 13.23 & 1140 \\
\hline 27.22 & 2.67 & 4.18 & 3.45 & 4.64 & & 47.52 & 38.98 & 55.65 & & 3.61 & -8.71 & 14.08 & 821 \\
\hline 27.67 & 2.67 & 4.01 & 3.28 & 4.80 & 0.00254 & 44.96 & 28.06 & 57.56 & 625 & 1.77 & $\begin{array}{l}-14.87 \\
\end{array}$ & 22.08 & 836 \\
\hline 27.67 & 3.45 & 4.07 & 3.09 & 5.07 & & 47.06 & 29.93 & 62.49 & & 2.57 & -26.69 & 31.38 & 0.22222 \\
\hline 27.67 & 4.22 & 4.02 & 3.03 & 4.82 & 315 & 48.21 & 30.95 & 60.54 & 520 & 6.06 & -17.43 & 28.62 & 0.22575 \\
\hline 27.67 & 5.00 & 3.77 & 2.96 & 4.77 & 0.00829 & 44.29 & 29.49 & 62.31 & 50 & 4.17 & -22.74 & 34.61 & 0.27858 \\
\hline 27.67 & 5.78 & 3.99 & 3.18 & 5.04 & 0.00834 & 46.10 & 30.23 & 59.09 & 028 & -1.07 & -29.16 & 35.58 & 0.22779 \\
\hline 27.67 & 6.55 & 4.05 & 3.09 & 5.16 & 17 & 46.28 & 32.79 & 59.90 & & 2.25 & -21.94 & 29.77 & 235 \\
\hline 27.67 & 7.33 & 4.03 & 3.29 & 4.72 & 0.00265 & 46.06 & 34.28 & 57.82 & 0.04620 & 3.59 & -18.17 & 24.50 & 0.07219 \\
\hline 27.67 & 12.67 & 4.15 & 3.31 & 4.97 & 0.00254 & 46.70 & 35.17 & 59.59 & 0.03879 & 4.19 & -14.61 & 24.36 & 0.06052 \\
\hline 27.67 & 45 & 4.18 & 3.30 & 4.82 & 36 & 46.51 & 35.56 & 59.17 & 01 & 6.49 & -10.40 & 30.06 & 399 \\
\hline 27.67 & 14.22 & 4.04 & 3.37 & 4.85 & 0.00806 & 44.66 & 34.04 & 56.16 & 0.13148 & 9.28 & -10.45 & 28.61 & 0.20479 \\
\hline 27.67 & 15.00 & 4.01 & 3.26 & 4.67 & 0.00790 & 45.09 & 33.38 & 58.53 & 0.13778 & 1.52 & -18.34 & 26.43 & 0.20541 \\
\hline
\end{tabular}




\begin{tabular}{|c|c|c|c|c|c|c|c|c|c|c|c|c|c|}
\hline 27.67 & 15.78 & 4.27 & 3.40 & 5.03 & 0.00755 & 48.64 & 38.05 & 59.93 & 0.11771 & 3.19 & $\begin{array}{l}-16.42 \\
\end{array}$ & 29.52 & 0.19439 \\
\hline 27.67 & 16.55 & 4.22 & 3.43 & 5.05 & 0.00795 & 46.98 & 33.23 & 57.90 & 0.13471 & 4.45 & -14.32 & 24.72 & 0.19771 \\
\hline 27.67 & 7.33 & .12 & 3.34 & 4.97 & 0.00257 & 45.03 & 2.04 & 57.47 & 0.04501 & 3.18 & -15.71 & 23.52 & .06586 \\
\hline 28.07 & 7.33 & 3.93 & 3.51 & 4.44 & 0.02755 & 46.77 & 37.96 & 54.65 & 0.40772 & 4.41 & -8.75 & 18.63 & .69147 \\
\hline 28.07 & 17.33 & 3.98 & 3.32 & 4.56 & 0.02894 & 44.56 & 35.19 & 53.74 & 0.50467 & 1.77 & -13.28 & 20.50 & .62941 \\
\hline 28.47 & 7.33 & 4.11 & 3.47 & 4.74 & 0.02946 & 45.61 & 35.46 & 52.11 & .36924 & 1.87 & -11.05 & 12.09 & .58497 \\
\hline 28.47 & 17.33 & 4.21 & 3.57 & 4.69 & 0.02796 & 43.62 & 36.70 & 53.04 & 0.40453 & 2.85 & -9.05 & 17.66 & 0.56265 \\
\hline 28.88 & 7.33 & 4.22 & 3.70 & 4.78 & 0.02608 & 42.20 & 31.06 & 49.63 & 0.42503 & 3.92 & -12.84 & 17.63 & 0.70926 \\
\hline 28.88 & 17.33 & 4.59 & 3.85 & 5.21 & 0.02757 & 44.45 & 34.46 & 52.65 & 0.38740 & 6.73 & -6.50 & 19.04 & 0.57612 \\
\hline 29.28 & 7.33 & 4.09 & 3.65 & 4.78 & 0.02419 & 39.37 & 30.39 & 49.41 & 0.40282 & 5.29 & -11.40 & 22.05 & 0.67110 \\
\hline 29.28 & 17.33 & 4.48 & 3.90 & 5.07 & 0.02461 & 37.96 & 30.02 & 45.81 & 0.37516 & 3.02 & -14.05 & 22.37 & 0.73648 \\
\hline 29.68 & 7.33 & 4.07 & 3.48 & 4.63 & 0.02659 & 39.44 & 31.12 & 48.62 & 637 & 6.24 & -7.16 & 20.12 & 0.61487 \\
\hline 29.68 & 17.33 & 4.52 & 3.96 & 5.18 & 0.02788 & 38.52 & 27.46 & 46.03 & 0.41995 & 4.68 & -21.45 & 18.48 & 0.71588 \\
\hline 30.10 & 2.67 & 4.13 & 3.48 & 4.76 & 0.00528 & 38.28 & 29.97 & 49.13 & & 3.74 & -10.39 & 16.41 & 0.13040 \\
\hline 30.10 & 3.45 & 4.13 & 3.54 & 4.71 & 0.00735 & 38.64 & 29.63 & 47.79 & & 3.98 & -11.44 & 21.52 & 0.18673 \\
\hline 30.10 & 4.22 & 4.10 & 3.54 & 4.62 & 0.00721 & 40.61 & 30.61 & 48.63 & 437 & 5.77 & -8.49 & 20.31 & 0.19043 \\
\hline 30.10 & 5.00 & 3.95 & 3.41 & 4.49 & 0.00764 & 38.02 & 29.68 & 47.16 & 726 & 6.32 & -10.17 & 19.93 & 0.20941 \\
\hline 30.10 & 5.78 & 4.04 & 3.55 & 4.68 & 0.00 & 38.90 & 29.63 & 48.12 & & -0.76 & -13.29 & 12.58 & .19325 \\
\hline 30.10 & 6.55 & 4.07 & 3.55 & 4.65 & 0.00709 & 39.89 & 31.15 & 47.70 & 96 & 3.56 & -9.09 & 17.65 & 0.19336 \\
\hline 30.10 & 7.33 & 4.05 & 3.50 & 4.75 & 0.00 & 39.42 & 30.24 & 47.82 & 95 & 4.49 & -9.32 & 19.49 & 088 \\
\hline 30.10 & 2.67 & 4.17 & 3.47 & 4.85 & 0.00 & 39.50 & 30.85 & 49.51 & & 3.01 & -14.82 & 17.49 & 2815 \\
\hline 30.10 & 3.45 & 4.19 & 3.56 & 4.86 & 0.00733 & 39.69 & 31.27 & 46.27 & & 3.39 & -9.07 & 17.45 & 0.17970 \\
\hline 30.10 & 14.22 & 4.21 & 3.65 & 4.96 & 0.0 & 38.49 & 30.23 & 48.49 & & 7.22 & -11.22 & 19.55 & .20510 \\
\hline 30.10 & .00 & 4.07 & 3.62 & 4.60 & 0.0 & 35.59 & 25.79 & 43.12 & & 3.62 & -17.20 & 22.08 & .26156 \\
\hline 30.10 & 15.78 & 4.28 & 3.50 & 4.84 & 0.00777 & 40.16 & 31.17 & 46.26 & 46 & 2.69 & -13.99 & 17.54 & 0.19155 \\
\hline 30.10 & .55 & 4.39 & 3.84 & 4.95 & 77 & 39.41 & 28.27 & 46.15 & & 4.31 & $\begin{array}{l}-10.69 \\
\end{array}$ & 22.19 & 9075 \\
\hline 30.10 & 17.33 & 4.42 & 3.83 & 5.07 & 0.00590 & 38.55 & 28.89 & 47.10 & & 4.15 & -11.61 & 20.75 & 055 \\
\hline 30.11 & 2.67 & 4.14 & 3.40 & 5.03 & 0.00541 & 36.40 & 21.13 & 47.05 & 45 & 1.45 & -16.98 & 21.15 & 0.15402 \\
\hline 30.11 & 3.45 & 4.16 & 3.33 & 5.11 & 61 & 37.57 & 23.45 & 47.44 & & 4.13 & -21.12 & 22.08 & 411 \\
\hline 30.11 & 4.22 & 4.12 & 3.30 & 4.80 & 0.0 & 39.73 & 26.73 & 49.50 & & 5.71 & -14.81 & 23.27 & 0.21474 \\
\hline 30.11 & 5.00 & 4.17 & 3.17 & 5.10 & 0.00869 & 39.79 & 26.48 & 52.74 & 510 & 5.68 & -13.10 & 34.97 & 0.24780 \\
\hline 30.11 & 78 & 4.03 & 3.38 & 4.96 & 61 & 37.68 & 24.33 & 52.35 & & 3.20 & -17.08 & 25.68 & 431 \\
\hline 30.11 & 6.55 & 4.14 & 3.29 & 4.99 & 390 & 39.69 & 24.41 & 54.17 & 79 & 0.74 & -15.88 & 22.32 & 0.24295 \\
\hline 30.11 & 7.33 & 4.09 & 3.69 & 4.42 & & 39.56 & 35.44 & 43.37 & & 4.79 & -1.13 & 10.44 & .31651 \\
\hline 30.11 & 67 & 4.21 & 3.48 & 4.97 & 58 & 40.75 & 30.39 & 50.81 & & 2.31 & -11.93 & 16.48 & 2787 \\
\hline 30.11 & 45 & 4.28 & 3.47 & 5.14 & 85 & 40.68 & 27.02 & 49.80 & & 2.28 & -13.09 & 21.36 & 184 \\
\hline 30.11 & 14.22 & 4.24 & 3.54 & 5.24 & 0.00864 & 39.81 & 25.07 & 50.08 & & 6.04 & -11.43 & 27.87 & 0.23689 \\
\hline 30.11 & .00 & 4.25 & 3.48 & 5.16 & 97 & 38.63 & 28.23 & 52.15 & 32 & 7.57 & -9.31 & 24.32 & .24095 \\
\hline 30.11 & .78 & 4.21 & 3.50 & 5.10 & & 37.83 & 24.15 & 48.84 & & 1.49 & -12.70 & 22.26 & 0.25222 \\
\hline 30.11 & 16.55 & 4.45 & 3.47 & 5.29 & & 41.68 & 30.30 & 48.92 & & 2.67 & -10.16 & 20.60 & 222 \\
\hline 30.11 & 17.33 & 4.48 & 4.19 & 4.78 & 55 & 40.24 & 37.86 & 43.69 & 99 & 4.30 & -0.52 & 10.18 & .24054 \\
\hline 30.20 & 7.33 & 3.71 & 3.48 & 3.88 & & 35.77 & 34.13 & 38.34 & & 6.22 & 2.26 & 12.42 & .43387 \\
\hline 30.20 & 17.33 & 4.14 & 4.04 & 4.21 & 16 & 35.55 & 33.78 & 37.86 & & 3.07 & 0.83 & 6.68 & 1.26390 \\
\hline 30.29 & 7.33 & 3.95 & 3.82 & 4.12 & 21 & 37.23 & 35.01 & 39.50 & 44 & 4.51 & 1.52 & 9.62 & 1.27114 \\
\hline 30.29 & 17.33 & 4.35 & 4.22 & 4.47 & 44 & 36.23 & 35.07 & 37.26 & & 4.12 & 0.83 & 7.70 & 0183 \\
\hline 30.38 & 7.33 & 4.06 & 3.95 & 4.15 & & 39.61 & 36.60 & 42.94 & & 5.02 & 1.43 & 7.39 & 805 \\
\hline 30.38 & 17.33 & 4.38 & 4.19 & 4.64 & 339 & 39.43 & 37.51 & 40.66 & 352 & 4.42 & 2.47 & 6.36 & 0.60855 \\
\hline 30.46 & 7.33 & 4.01 & 3.91 & 4.18 & & 38.86 & 37.15 & 40.72 & & 7.55 & 4.69 & 10.20 & 9949 \\
\hline 30.46 & 17.33 & 4.46 & 4.28 & 4.60 & 0.03957 & 40.76 & 39.55 & 42.07 & 330 & 3.53 & 0.81 & 7.79 & 0.97422 \\
\hline 30.55 & 7.33 & 4.11 & 3.90 & 4.22 & 0.04446 & 39.78 & 38.23 & 41.49 & & 5.80 & 2.96 & 9.03 & .81617 \\
\hline 30.55 & 17.33 & 4.49 & 4.24 & 4.60 & 268 & 39.77 & 38.13 & 42.32 & 656 & 5.27 & 3.61 & 6.48 & 0.47239 \\
\hline 30.64 & 2.67 & 3.94 & 3.72 & 4.10 & 0.00802 & 33.27 & 29.90 & 35.80 & 357 & 2.35 & -4.98 & 8.10 & 0.25930 \\
\hline 30.64 & 3.45 & 3.91 & 3.70 & 4.16 & 0.01690 & 34.98 & 31.19 & 38.16 & 542 & 3.91 & -0.26 & 9.52 & 0.36693 \\
\hline 30.64 & 4.22 & 3.90 & 3.69 & 4.08 & 10 & 36.12 & 33.21 & 38.61 & & 4.89 & -0.14 & 12.35 & 0.45479 \\
\hline 30.64 & 5.00 & 3.81 & 3.62 & 3.97 & 0.01563 & 34.21 & 29.33 & 38.49 & 0.39011 & 5.90 & -4.93 & 12.80 & 0.63850 \\
\hline 30.64 & 5.78 & 3.85 & 3.59 & 4.16 & 0.01888 & 35.30 & 28.56 & 39.54 & 522 & 1.49 & -6.83 & 9.97 & 0.65891 \\
\hline 30.64 & 6.55 & 3.84 & 3.59 & 4.14 & 56 & 35.85 & 32.62 & 39.75 & & 5.06 & 1.89 & 9.77 & 0.35414 \\
\hline 30.64 & 7.33 & 3.78 & 3.59 & 4.01 & 0.01509 & 36.05 & 31.11 & 39.65 & 0.24652 & 6.11 & 0.48 & 11.36 & 0.36205 \\
\hline 30.64 & 12.67 & 3.98 & 3.72 & 4.21 & 0.00917 & 37.09 & 33.71 & 40.01 & 0.12854 & 0.49 & -4.42 & 5.84 & 0.23957 \\
\hline
\end{tabular}




\begin{tabular}{|c|c|c|c|c|c|c|c|c|c|c|c|c|c|}
\hline 30.64 & 3.45 & 3.95 & 3.77 & 4.20 & 0.01945 & 36.87 & 33.74 & 39.88 & 0.24541 & 1.20 & -3.00 & 5.60 & 0.37589 \\
\hline 30.64 & 4.22 & 4.00 & 3.84 & 4.24 & 0.01781 & 36.28 & 31.87 & 40.25 & 0.27099 & 5.27 & -0.33 & 11.44 & 0.55413 \\
\hline 30.64 & 5.00 & 3.90 & 3.67 & 4.11 & 0.01629 & 32.39 & 28.66 & 34.83 & 0.24173 & 3.72 & -6.15 & 13.50 & .81666 \\
\hline 30.64 & 5.78 & 4.06 & 3.78 & 4.31 & 0.01758 & 36.36 & 33.84 & 38.95 & 0.22653 & 3.08 & -3.62 & 9.34 & .47092 \\
\hline 30.64 & 6.55 & 4.18 & 3.86 & 4.46 & 0.01980 & 35.31 & 31.68 & 38.97 & 0.26279 & 4.13 & 0.32 & 10.27 & 0.37506 \\
\hline 30.64 & 17.33 & 4.19 & 3.65 & 4.41 & 0.01977 & 35.71 & 32.63 & 38.99 & 0.19047 & 3.40 & -0.10 & 10.16 & 0.34241 \\
\hline 30.69 & 2.67 & 3.97 & 3.78 & 4.10 & 0.04102 & 34.01 & 32.63 & 36.48 & 0.63217 & 1.03 & -0.44 & 2.79 & 0.43971 \\
\hline 30.69 & 12.67 & 3.93 & 3.77 & 4.10 & 0.04463 & 36.95 & 35.51 & 39.19 & 0.47938 & 1.10 & -1.51 & 6.28 & 1.01510 \\
\hline 30.78 & 2.67 & 3.93 & 3.72 & 4.05 & 0.04061 & 33.90 & 32.29 & 35.00 & 0.44801 & 3.28 & -1.47 & 6.96 & 1.02819 \\
\hline 30.78 & 12.67 & 3.93 & 3.83 & 4.12 & 0.03623 & 37.71 & 35.33 & 40.03 & 0.63292 & 1.15 & -1.17 & 5.85 & 0.98953 \\
\hline 30.86 & 2.67 & 4.09 & 3.91 & 4.28 & 0.05244 & 31.36 & 28.70 & 33.44 & 0.67750 & 3.48 & 0.26 & 7.97 & \\
\hline 30.86 & 12.67 & 4.15 & 3.93 & 4.55 & 0.07597 & 35.53 & 31.85 & 40.23 & 1.06985 & 0.45 & -3.18 & 4.12 & 1.15833 \\
\hline 30.94 & 2.67 & 4.47 & 4.41 & 4.55 & 0.02024 & 26.96 & 26.00 & 28.31 & 0.36403 & 0.15 & -2.44 & 3.88 & 1.00707 \\
\hline 30.94 & 2.67 & 4.49 & 4.32 & 4.61 & 0.03895 & 31.58 & 30.18 & 33.32 & 533 & 1.48 & -0.05 & 3.35 & \\
\hline 31.03 & 2.67 & 4.50 & 4.40 & 4.56 & 0.02289 & 27.15 & 25.35 & 28.83 & 0.4 & 3.49 & 1.27 & 5.89 & \\
\hline 31.03 & 12.67 & 4.55 & 4.48 & 4.64 & 0.02325 & 32.83 & 29.18 & 36.41 & 1.00423 & 0.51 & -4.60 & 3.91 & 1.0 \\
\hline 31.11 & 2.67 & 4.53 & 4.37 & 4.71 & 0.01 & 27.83 & 25.07 & 31.34 & 0.1 & -0.01 & -10.92 & 9.94 & 592 \\
\hline 31.11 & 3.45 & 4.55 & 4.31 & 4.79 & 0.00796 & 28.49 & 24.25 & 33.97 & 399 & 6.09 & -3.63 & 14.23 & 0.3 \\
\hline 31.11 & 4.22 & 4.55 & 4.32 & 4.81 & 0.00852 & 30.42 & 26.47 & 33.67 & 0.13250 & 7.78 & 1.11 & 13.34 & 0.2 \\
\hline 31.11 & 5.00 & 4.37 & 4.15 & 4.61 & 0.0 & 29.34 & 22.97 & 33.62 & 51 & 3.74 & -8.18 & 14.56 & 113 \\
\hline 31.11 & 5.78 & 4.47 & 4.09 & 4.75 & 0.0 & 31.25 & 26.12 & 35.35 & 0.17399 & 3.47 & -3.04 & 10.63 & 771 \\
\hline 31.11 & 6.55 & 4.45 & 4.16 & 4.78 & 0.01074 & 30.97 & 25.79 & 35.26 & 0.17211 & 4.36 & -2.34 & 13.87 & 0.27797 \\
\hline 31.11 & 7.33 & 4.46 & 4.13 & 4.76 & & 30.29 & 25.71 & 33.96 & & 5.34 & -2.66 & 13.71 & \\
\hline 31.11 & 12.67 & 4.48 & 4.20 & 4.67 & 0.0 & 31.85 & 28.40 & 35.28 & 0.2 & 0.97 & -5.07 & 7.32 & 0.3 \\
\hline 31.11 & 13.45 & 4.54 & 4.33 & 4.86 & 0.0 & 32.02 & 28.17 & 35.76 & 34 & 1.87 & -4.13 & 8.08 & 0.2 \\
\hline 31.11 & .22 & 4.56 & 4.33 & 4.82 & & 30.04 & 23.83 & 35.36 & & 6.07 & -0.46 & 14.96 & \\
\hline 31.11 & 5.00 & 4.55 & 4.31 & 4.83 & 0.0 & 29.34 & 24.48 & 35.85 & 30 & 0.07 & -7.02 & 7.00 & 0.2 \\
\hline 31.11 & .78 & 4.72 & 4.49 & 4.96 & 0.0 & 30.48 & 27.19 & 34.17 & & 3.15 & -5.08 & 8.27 & 350 \\
\hline 31.11 & .55 & 4.70 & 4.48 & 4.96 & & 29.95 & 26.93 & 32.63 & & 2.98 & -2.68 & 9.56 & \\
\hline 31.11 & 17.33 & 4.75 & 4.54 & 4.97 & 63 & 29.42 & 26.36 & 31.82 & & 2.22 & -4.65 & 11.66 & 0.2 \\
\hline 31.19 & 7.33 & 4.41 & 4.28 & 4.63 & 0.0 & 30.18 & 26.01 & 32.20 & & 6.94 & 1.96 & 17.01 & 319 \\
\hline 31.19 & 17.33 & 4.72 & 4.65 & 4.77 & 71 & 30.53 & 29.87 & 32.18 & & 0.50 & -1.35 & 3.70 & 88 \\
\hline 31.28 & 7.33 & 4.40 & 4.31 & 4.49 & 16 & 30.90 & 29.03 & 32.32 & & 5.34 & 2.93 & 7.64 & 362 \\
\hline 31.28 & .33 & 4.77 & 4.72 & 4.87 & & 29.73 & 27.41 & 32.00 & & 3.17 & 1.00 & 5.26 & \\
\hline 31.36 & 7.33 & 4.19 & 4.01 & 4.36 & 57 & 31.09 & 27.79 & 34.89 & 25 & 3.54 & 0.44 & 6.77 & 742 \\
\hline 31.36 & 17.33 & 4.54 & 4.26 & 4.73 & & 30.73 & 29.49 & 31.86 & & 3.72 & -0.07 & 5.00 & 341 \\
\hline 31.44 & 7.33 & 4.16 & 4.00 & 4.27 & & 33.97 & 30.20 & 38.32 & & 5.23 & 2.47 & 7.63 & 104 \\
\hline 31.44 & 17.33 & 4.40 & 4.20 & 4.57 & 62 & 31.14 & 30.17 & 31.98 & 0.2 & 2.44 & -0.08 & 6.23 & 498 \\
\hline 31.53 & 7.33 & 4.24 & 4.06 & 4.38 & & 29.27 & 25.84 & 33.20 & & 4.27 & -2.31 & 7.44 & 992 \\
\hline 31.53 & .33 & 4.69 & 4.43 & 4.81 & & 28.88 & 25.87 & 30.49 & & 3.82 & -0.85 & 7.52 & \\
\hline 31.61 & 2.67 & 4.98 & 4.74 & 5.24 & 48 & 28.19 & 25.82 & 31.22 & 62 & -0.87 & -8.37 & 5.55 & 010 \\
\hline 31.61 & 3.45 & 4.98 & 4.77 & 5.18 & & 29.28 & 26.78 & 31.90 & & 4.17 & -2.65 & 9.91 & 903 \\
\hline 31.61 & 4.22 & 4.93 & 4.79 & 5.08 & & 30.66 & 28.21 & 33.39 & & 6.18 & -0.35 & 11.65 & 115 \\
\hline 31.61 & 5.00 & 4.72 & 4.50 & 4.94 & 42 & 28.88 & 24.92 & 33.25 & 64 & 3.86 & -5.60 & 13.64 & 0.62753 \\
\hline 31.61 & 5.78 & 4.89 & 4.71 & 5.14 & 0.01227 & 31.39 & 25.66 & 35.26 & 939 & -0.42 & -8.02 & 6.87 & 0.43004 \\
\hline 31.61 & 6.55 & 4.93 & 4.69 & 5.12 & & 30.47 & 25.52 & 33.58 & 23 & 3.07 & -3.96 & 12.37 & 426 \\
\hline 31.61 & 7.33 & 4.89 & 4.70 & 5.21 & & 30.22 & 26.99 & 33.84 & 0.12125 & 4.08 & -3.28 & 10.65 & 0.23566 \\
\hline 31.61 & .67 & 5.03 & 4.76 & 5.25 & 29 & 31.73 & 27.97 & 35.45 & & 1.36 & -3.31 & 8.98 & 0.26240 \\
\hline 31.61 & 13.45 & 5.00 & 4.80 & 5.23 & 0.01229 & 31.17 & 27.51 & 35.11 & 145 & 3.12 & -4.36 & 12.96 & 0.48643 \\
\hline 31.61 & 14.22 & 4.90 & 4.74 & 5.16 & 0.01409 & 29.09 & 24.64 & 31.78 & 861 & 7.40 & -0.57 & 16.11 & 0.44811 \\
\hline 31.61 & 15.00 & 4.91 & 4.66 & 5.17 & 0.0 & 28.52 & 24.46 & 33.21 & 0.2 & 0.32 & -9.61 & 7.13 & 0.40254 \\
\hline 31.61 & 15.78 & 5.13 & 4.92 & 5.43 & 0.01 & 30.45 & 28.47 & 32.94 & 0.13502 & 3.05 & -2.16 & 8.43 & 0.32061 \\
\hline 31.61 & 16.55 & 5.20 & 4.97 & 5.51 & 0.01453 & 29.79 & 27.61 & 32.78 & 0.14614 & 3.71 & -1.40 & 9.23 & 0.32794 \\
\hline 31.61 & 17.33 & 5.19 & 4.80 & 5.45 & & 29.55 & 26.73 & 33.64 & & 3.11 & -3.05 & 8.52 & 0.18270 \\
\hline 31.67 & 2.67 & 5.02 & 4.86 & 5.21 & 0.03241 & 28.01 & 25.79 & 29.32 & 0.33968 & 0.36 & -5.30 & 5.56 & 0.90789 \\
\hline 31.67 & 12.67 & 5.07 & 4.85 & 5.31 & 0.03817 & 32.26 & 29.82 & 33.75 & 0.33921 & -0.11 & -7.13 & 3.49 & 0.95025 \\
\hline 31.72 & 2.67 & 4.98 & 4.83 & 5.09 & 0.02954 & 27.75 & 25.73 & 29.28 & 0.34604 & 1.34 & -2.79 & 4.32 & 0.72541 \\
\hline 31.72 & 12.67 & 5.01 & 4.91 & 5.21 & 0.03714 & 32.09 & 29.67 & 34.82 & 0.47429 & 0.67 & -2.13 & 6.49 & 0.88657 \\
\hline 31.78 & 2.67 & 5.19 & 4.97 & 5.44 & 0.04830 & 29.60 & 27.06 & 31.31 & 0.49089 & -0.21 & -2.81 & 3.67 & 0.65579 \\
\hline
\end{tabular}




\begin{tabular}{|c|c|c|c|c|c|c|c|c|c|c|c|c|c|}
\hline 31.78 & 12.67 & 5.16 & 4.96 & 5.40 & 0.04159 & 33.46 & 29.89 & 35.26 & 0.44867 & 0.33 & -3.65 & 3.04 & 0.65144 \\
\hline 31.84 & 2.67 & 4.92 & 4.70 & 5.04 & 0.03751 & 27.70 & 25.54 & 29.58 & 0.41265 & -0.72 & -3.01 & 2.44 & 0.51194 \\
\hline 31.84 & 12.67 & 4.98 & 4.83 & 5.27 & 0.04232 & 32.50 & 30.01 & 34.97 & 0.55041 & 0.58 & -5.82 & 4.04 & .97241 \\
\hline 31.89 & 2.67 & 5.49 & 5.34 & 5.70 & 0.03399 & 31.43 & 29.45 & 33.48 & 0.44003 & -0.41 & -2.95 & 2.54 & .56396 \\
\hline 31.89 & 2.67 & 5.60 & 5.51 & 5.74 & 0.02253 & 36.05 & 34.32 & 38.73 & 0.44535 & 1.64 & -1.99 & 5.07 & 0.69429 \\
\hline 31.95 & 2.67 & 5.45 & 5.24 & 5.70 & 0.00915 & 31.62 & 29.34 & 34.78 & 0.10069 & 0.01 & -4.74 & 5.88 & 0.20489 \\
\hline 31.95 & 3.45 & 5.49 & 5.26 & 5.67 & 0.00966 & 33.46 & 29.41 & 37.48 & 0.16495 & 5.40 & -3.63 & 10.92 & 0.33301 \\
\hline 31.95 & 4.22 & 5.44 & 5.20 & 5.67 & 0.00922 & 34.76 & 32.64 & 38.43 & 0.11779 & 7.64 & 2.05 & 12.86 & 0.22882 \\
\hline 31.95 & 5.00 & 5.22 & 5.04 & 5.52 & 0.01052 & 33.07 & 29.03 & 35.70 & 0.13643 & 10.17 & 5.47 & 15.45 & 0.23071 \\
\hline 31.95 & 5.78 & 5.13 & 4.89 & 5.38 & 0.01129 & 31.97 & 27.13 & 37.00 & 0.19214 & 5.56 & -4.25 & 13.65 & 0.56460 \\
\hline 31.95 & 6.55 & 5.41 & 5.01 & 5.78 & 0.01 & 34.55 & 31.07 & 38.03 & 0.14241 & 2.10 & -2.81 & 9.21 & 0.27352 \\
\hline 31.95 & 7.33 & 5.42 & 5.23 & 5.62 & 0.01074 & 34.65 & 31.01 & 37.78 & 0.16712 & 4.13 & -1.00 & 9.19 & 0.25270 \\
\hline 31.95 & 12.67 & 5.57 & 5.36 & 5.80 & 0.00918 & 35.88 & 32.86 & 38.90 & 0.11877 & 1.57 & -4.37 & 7.11 & 0.21632 \\
\hline 31.95 & 45 & 5.57 & 5.37 & 5.81 & 0.0 & 35.08 & 30.80 & 38.31 & 0.14756 & 2.52 & -2.86 & 10.25 & 0.27135 \\
\hline 31.95 & .22 & 5.36 & 5.17 & 5.61 & 0.01 & 32.02 & 28.49 & 36.18 & 0.17487 & 8.20 & 0.12 & 14.33 & 0.28659 \\
\hline 31.95 & 15.00 & 5.24 & 4.98 & 5.49 & 0.01006 & 30.32 & 27.22 & 34.01 & 0.14273 & 0.18 & -8.05 & 13.00 & 0.39833 \\
\hline 31.95 & .78 & 5.41 & 5.09 & 5.79 & 0.01 & 32.08 & 28.19 & 35.64 & 0.20484 & -0.84 & -6.95 & 5.73 & 985 \\
\hline 31.95 & 16.55 & 5.76 & 5.49 & 6.03 & 0.0 & 34.33 & 31.90 & 37.24 & 0.12508 & 2.17 & -4.09 & 8.40 & 565 \\
\hline 31.95 & 17.33 & 5.73 & 5.49 & 5.99 & 0.01205 & 33.53 & 30.82 & 36.36 & 0.14449 & 2.32 & -3.32 & 6.90 & 0.21318 \\
\hline 32.02 & 7.33 & 5.45 & 5.21 & 5.63 & 0.0 & 34.28 & 30.52 & 36.40 & 599 & 4.72 & 1.26 & 7.45 & 304 \\
\hline 32.02 & 17.33 & 5.72 & 5.50 & 5.88 & 0.0 & 33.45 & 31.26 & 34.56 & 0.31450 & 1.72 & -1.22 & 5.62 & 0.5 \\
\hline 32.10 & 7.33 & 5.49 & 5.26 & 5.65 & 0.03 & 34.66 & 33.40 & 37.53 & 0.32284 & 4.27 & -1.49 & 7.60 & 0.78455 \\
\hline 32.10 & .33 & 5.85 & 5.63 & 6.08 & & 33.96 & 32.56 & 35.74 & 015 & 2.62 & 0.73 & 4.26 & 98 \\
\hline 32.18 & 7.33 & 5.47 & 5.36 & 5.59 & 0.0 & 34.80 & 32.94 & 37.36 & 0.38846 & 4.26 & -1.57 & 8.41 & 480 \\
\hline 32.18 & 17.33 & 5.88 & 5.71 & 6.04 & 0.03 & 34.12 & 32.23 & 36.46 & 0.36408 & 3.71 & -1.18 & 8.63 & 0.7 \\
\hline 32.25 & 7.33 & 5.50 & 5.36 & 5.61 & & 35.64 & 32.89 & 38.07 & 149 & 3.87 & -0.03 & 7.34 & 97 \\
\hline 32.25 & 17.33 & 5.85 & 5.63 & 6.01 & 0.0 & 34.84 & 33.49 & 35.72 & 415 & 3.86 & 0.49 & 6.98 & 0.6 \\
\hline 32.33 & 7.33 & 5.52 & 5.37 & 5.67 & 0.0 & 35.03 & 32.88 & 36.34 & 123 & 3.06 & -0.03 & 6.10 & 330 \\
\hline 32.33 & .33 & 5.83 & 5.68 & 5.95 & & 34.10 & 32.63 & 35.33 & 28 & 3.05 & 1.04 & 6.06 & 668 \\
\hline 32.41 & 2.67 & 5.34 & 5.10 & 5.57 & 99 & 31.86 & 27.83 & 34.79 & 543 & 1.19 & -3.29 & 6.21 & 0.2 \\
\hline 32.41 & 3.45 & 5.40 & 5.24 & 5.67 & 0.0 & 33.90 & 30.33 & 37.01 & 38 & 3.98 & -0.36 & 9.08 & 639 \\
\hline 32.41 & 4.22 & 5.50 & 5.21 & 5.81 & & 34.27 & 31.54 & 39.03 & & 3.79 & -1.92 & 9.44 & 873 \\
\hline 32.41 & 5.00 & 5.56 & 5.33 & 5.78 & & 34.76 & 31.50 & 37.65 & 82 & 5.81 & -1.19 & 13.42 & 469 \\
\hline 32.41 & 5.78 & 5.44 & 5.09 & 5.80 & & 33.88 & 28.85 & 38.55 & & 7.33 & -7.18 & 12.85 & 880 \\
\hline 32.41 & 6.55 & 5.52 & 5.22 & 5.80 & 25 & 34.77 & 30.12 & 38.20 & 730 & 1.06 & -7.18 & 7.14 & 323 \\
\hline 32.41 & 7.33 & 5.51 & 5.29 & 5.71 & & 34.78 & 32.22 & 38.04 & & 3.06 & -1.03 & 10.14 & 355 \\
\hline 32.41 & 67 & 5.52 & 5.30 & 5.76 & & 36.66 & 33.11 & 41.09 & 559 & 1.21 & -3.76 & 4.99 & 570 \\
\hline 32.41 & .45 & 5.55 & 5.27 & 5.81 & & 35.69 & 32.66 & 38.61 & 80 & 2.75 & -1.90 & 8.85 & 629 \\
\hline 32.41 & .22 & 5.65 & 5.46 & 5.87 & & 35.86 & 32.04 & 39.24 & 203 & 2.84 & -3.65 & 9.23 & 679 \\
\hline 32.41 & .00 & 5.65 & 5.33 & 6.00 & & 34.21 & 28.86 & 38.73 & & 5.97 & -2.56 & 12.97 & \\
\hline 32.41 & .78 & 5.54 & 5.21 & 5.90 & 50 & 31.87 & 27.91 & 35.02 & 53 & 4.00 & -4.72 & 14.75 & 913 \\
\hline 32.41 & .55 & 5.88 & 5.62 & 6.11 & & 34.95 & 31.75 & 37.70 & & 2.32 & -4.25 & 7.40 & 0.22058 \\
\hline 32.41 & 17.33 & 5.88 & 5.64 & 6.13 & & 34.27 & 32.11 & 36.45 & & 2.53 & -1.64 & 6.70 & 0.22402 \\
\hline 32.48 & 2.67 & 5.36 & 5.33 & 5.39 & 07 & 32.32 & 31.16 & 33.05 & 56 & -0.16 & -1.23 & 0.46 & 0.53725 \\
\hline 32.48 & 12.67 & 5.50 & 5.45 & 5.56 & 0.0 & 36.73 & 34.05 & 38.38 & 463 & 2.38 & 0.03 & 4.62 & 1.32582 \\
\hline 32.56 & 2.67 & 5.32 & 5.26 & 5.38 & & 32.39 & 32.37 & 32.41 & & 1.73 & 1.03 & 2.43 & 877 \\
\hline 32.56 & 12.67 & 5.52 & 5.46 & 5.58 & & 37.33 & 36.76 & 37.90 & 0.57233 & -2.11 & -2.39 & -1.82 & 0.28643 \\
\hline 32.64 & 2.67 & 5.29 & 5.22 & 5.40 & 0. & 31.03 & 29.90 & 31.65 & 537 & 0.73 & 0.07 & 1.14 & 169 \\
\hline 32.64 & 12.67 & 5.43 & 5.38 & 5.50 & 02 & 35.78 & 33.62 & 37.43 & 1.12671 & 1.14 & -0.20 & 2.02 & 61 \\
\hline 32.71 & 2.67 & 5.24 & 5.23 & 5.24 & & 32.91 & 31.70 & 34.12 & 1.21180 & 3.23 & 2.40 & 4.05 & 0.82262 \\
\hline 32.71 & 12.67 & 5.49 & 5.47 & 5.51 & 0.0 & 36.89 & 36.80 & 36.97 & 0.0 & -1.98 & -2.46 & -1.49 & 0.48275 \\
\hline 32.79 & 2.67 & 5.32 & 5.31 & 5.32 & & 32.86 & 32.12 & 33.60 & 0.74199 & 0.86 & -0.35 & 2.06 & 1.20263 \\
\hline 32.79 & 12.67 & 5.60 & 5.54 & 5.66 & 0.05864 & 37.94 & 37.55 & 38.33 & 0.38875 & 3.00 & 2.75 & 3.26 & 0.25707 \\
\hline 32.87 & 2.67 & 5.29 & 5.09 & 5.54 & 0.00 & 29.03 & 26.68 & 33.03 & & 1.74 & -4.48 & 8.32 & 0.21489 \\
\hline 32.87 & 3.45 & 5.30 & 5.04 & 5.51 & 0.01173 & 29.99 & 27.55 & 33.03 & 0.16359 & 5.19 & -1.54 & 12.67 & 0.43701 \\
\hline 32.87 & 4.22 & 5.31 & 5.11 & 5.47 & 0.01004 & 32.17 & 30.16 & 35.08 & 0.15703 & 8.03 & 2.86 & 13.27 & 0.28299 \\
\hline 32.87 & 5.00 & 5.18 & 4.88 & 5.41 & 0.01453 & 30.52 & 25.14 & 34.34 & 0.25280 & 9.89 & 3.12 & 17.18 & 0.39421 \\
\hline 32.87 & 5.78 & 5.15 & 4.95 & 5.46 & 0.01286 & 30.43 & 26.35 & 35.39 & 0.24329 & 1.68 & -4.28 & 7.14 & 0.32268 \\
\hline 32.87 & 6.55 & 5.26 & 5.08 & 5.52 & 0.01131 & 32.30 & 29.25 & 36.14 & 0.19209 & 2.84 & -2.76 & 10.80 & 0.33289 \\
\hline
\end{tabular}




\begin{tabular}{|c|c|c|c|c|c|c|c|c|c|c|c|c|c|}
\hline 32.87 & 7.33 & 5.22 & 5.02 & 5.48 & 0.01235 & 31.80 & 28.96 & 34.54 & 0.15957 & 4.18 & -0.87 & 9.01 & 0.28840 \\
\hline 32.87 & 12.67 & 5.43 & 5.21 & 5.68 & 0.00856 & 33.26 & 28.44 & 37.18 & 0.13434 & -0.05 & -5.82 & 6.56 & 0.22175 \\
\hline 32.87 & .45 & 46 & 5.27 & 5.70 & 0.01270 & 33.10 & 28.88 & 35.26 & .16482 & 0.83 & -5.34 & 8.20 & .36166 \\
\hline 32.87 & .22 & 5.41 & 5.24 & 5.61 & 0.01325 & 31.07 & 27.27 & 35.47 & 0.23491 & 4.87 & -1.72 & 11.67 & .39243 \\
\hline 32.87 & 15.00 & 5.31 & 5.08 & 5.62 & 0.01467 & 27.88 & 23.49 & 32.09 & 0.23994 & -0.06 & -7.52 & 12.31 & .55829 \\
\hline 32.87 & .78 & 5.53 & 5.30 & 5.67 & 0.00989 & 31.52 & 28.04 & 34.12 & .18241 & -1.46 & -7.62 & 3.50 & .35535 \\
\hline 32.87 & 16.55 & 5.61 & 5.42 & 5.81 & 0.01112 & 31.40 & 28.55 & 34.20 & 0.15312 & 1.21 & -3.14 & 7.38 & 0.32962 \\
\hline 32.87 & 17.33 & 5.60 & 5.44 & 5.78 & 0.00956 & 30.66 & 27.69 & 33.40 & 0.12497 & 1.34 & -3.74 & 5.87 & 0.24640 \\
\hline 32.95 & 2.67 & 5.32 & 5.14 & 5.56 & 0.00757 & 28.97 & 26.11 & 31.83 & 0.10983 & 6.82 & -0.64 & 13.56 & 0.22314 \\
\hline 32.95 & 3.45 & 5.33 & 5.11 & 5.61 & 0.01142 & 30.36 & 27.04 & 33.21 & 0.15661 & 5.01 & -1.01 & 9.14 & 0.27799 \\
\hline 32.95 & 4.22 & 5.30 & 5.11 & 5.52 & 0.01006 & 31.53 & 28.01 & 33.99 & 0.15945 & 8.25 & -0.60 & 15.30 & 0.38525 \\
\hline 32.95 & 5.00 & 5.14 & 4.92 & 5.34 & 0.01349 & 28.66 & 25.19 & 33.44 & 0.22030 & 5.28 & -8.41 & 14.90 & 0.65213 \\
\hline 32.95 & 5.78 & 5.23 & 5.00 & 5.40 & 0.01086 & 30.73 & 26.72 & 34.53 & 0.16585 & 1.65 & -4.23 & 7.56 & 0.33888 \\
\hline 32.95 & 6.55 & 5.28 & 4.95 & 5.47 & 0.0 & 31.07 & 26.45 & 34.12 & 0.17987 & 3.30 & -1.79 & 9.87 & 0.36013 \\
\hline 32.95 & 7.33 & 5.27 & 4.97 & 5.53 & 0.0 & 31.14 & 26.77 & 34.74 & 309 & 3.61 & -5.15 & 13.59 & 813 \\
\hline 32.95 & 12.67 & 5.46 & 5.16 & 5.77 & 0.00931 & 32.41 & 28.38 & 35.94 & 0.12936 & 2.00 & -2.27 & 8.17 & 0.18891 \\
\hline 32.95 & 13.45 & 5.50 & 5.24 & 5.75 & 0.01247 & 32.08 & 29.06 & 34.93 & 0.19637 & 2.04 & -4.01 & 9.54 & 0.36696 \\
\hline 32.95 & .22 & 5.41 & 5.25 & 5.63 & 0.0 & 28.57 & 24.30 & 33.37 & 645 & 6.38 & -2.85 & 12.99 & 131 \\
\hline 32.95 & 15.00 & 5.45 & 5.23 & 5.70 & 0.01434 & 28.40 & 22.49 & 32.59 & 0.27024 & -0.62 & -6.28 & 5.71 & 0.32791 \\
\hline 32.95 & 15.78 & 5.62 & 5.37 & 5.94 & 0.0 & 30.95 & 28.14 & 34.68 & 570 & 2.42 & -3.73 & 8.85 & 04 \\
\hline 32.95 & 16.55 & 5.71 & 5.50 & 6.03 & 0.0 & 29.90 & 26.88 & 32.83 & 873 & 2.75 & -2.89 & 9.70 & 454 \\
\hline 32.95 & 17.33 & 5.71 & 5.46 & 6.00 & 0.00740 & 29.78 & 26.84 & 33.43 & 0.09406 & 2.11 & -4.83 & 7.94 & 0.16814 \\
\hline 33.31 & 7.33 & 5.27 & 5.06 & 5.43 & 0.0 & 31.09 & 27.96 & 32.62 & 15 & 3.69 & -2.12 & 8.49 & 492 \\
\hline 33.31 & 17.33 & 5.66 & 5.37 & 5.84 & 34 & 29.14 & 27.03 & 31.13 & 89 & 2.09 & -5.04 & 6.42 & 970 \\
\hline 33.66 & 7.33 & 5.97 & 5.04 & 6.48 & 0.1 & 27.60 & 22.04 & 35.54 & 274 & 5.32 & -1.15 & 16.82 & 064 \\
\hline 33.66 & 33 & 6.36 & 5.58 & 6.93 & & 25.93 & 19.56 & 32.10 & 956 & -0.19 & -5.29 & 5.12 & 52 \\
\hline 34.02 & 7.33 & 6.46 & 6.28 & 6.65 & 39 & 22.63 & 19.42 & 24.96 & 858 & 6.51 & 1.56 & 10.81 & 476 \\
\hline 34.02 & 17.33 & 6.84 & 6.70 & 6.99 & 0.0 & 21.23 & 18.86 & 22.92 & 416 & -2.17 & -7.05 & 2.69 & 440 \\
\hline 34.37 & 33 & 6.44 & 6.27 & 6.60 & & 22.84 & 20.64 & 24.50 & 951 & 6.02 & 1.02 & 13.87 & 721 \\
\hline 34.37 & 17.33 & 6.83 & 6.62 & 6.99 & 0.0 & 21.27 & 18.01 & 23.29 & 237 & -0.84 & -11.26 & 5.15 & 046 \\
\hline 34.73 & 7.33 & 6.45 & 6.31 & 6.58 & 0.01 & 22.42 & 19.88 & 25.24 & 513 & 6.01 & 0.68 & 10.31 & 335 \\
\hline 34.73 & 33 & 6.84 & 6.69 & 6.96 & & 20.78 & 18.23 & 23.78 & 90 & -1.88 & -7.34 & 4.33 & 89 \\
\hline 35.08 & 2.67 & 6.57 & 5.91 & 7.42 & 04 & 20.52 & 10.90 & 33.65 & 592 & 2.86 & -26.28 & 35.93 & 783 \\
\hline 35.08 & 3.45 & 6.57 & 5.74 & 7.46 & & 22.56 & 10.05 & 36.41 & 21 & 12.27 & -19.97 & 9.82 & 363 \\
\hline 35.08 & 22 & 6.55 & 5.97 & 7.54 & & 24.31 & 11.10 & 37.94 & 32 & 13.99 & 13.62 & 6.03 & 177 \\
\hline 35.08 & 5.00 & 6.44 & 5.79 & 7.16 & & 23.34 & 11.01 & 38.09 & 55 & 15.55 & -14.58 & 55.37 & 171 \\
\hline 35.08 & 5.78 & 6.37 & 5.52 & 7.46 & & 21.97 & 11.30 & 34.01 & 66 & 6.56 & -21.19 & 44.52 & 047 \\
\hline 35.08 & .55 & 6.44 & 5.47 & 7.09 & & 23.96 & 11.18 & 36.54 & 08 & 7.13 & -15.10 & 3.94 & 359 \\
\hline 35.08 & 7.33 & 6.44 & 5.53 & 7.78 & & 22.90 & 8.54 & 42.19 & 68 & 6.88 & -29.45 & 50.86 & 130 \\
\hline 35.08 & .67 & 6.53 & 5.93 & 7.40 & & 24.46 & 12.67 & 34.38 & & -1.78 & -23.78 & 23.98 & \\
\hline 35.08 & .45 & 6.55 & 5.98 & 7.06 & 81 & 24.04 & 15.85 & 31.61 & 10 & -0.05 & -23.37 & 3.64 & 043 \\
\hline 35.08 & 22 & 6.55 & 5.95 & 7.12 & & 21.77 & 13.82 & 32.82 & & 2.31 & -27.40 & 25.47 & 796 \\
\hline 35.08 & .00 & 6.53 & 5.98 & 7.03 & & 19.45 & 13.26 & 28.04 & 596 & -2.09 & -29.23 & 26.58 & 0.29442 \\
\hline 35.08 & .78 & 6.66 & 6.09 & 7.32 & 05 & 21.73 & 14.04 & 30.48 & 69 & -2.80 & -24.90 & 27.00 & .26004 \\
\hline 35.08 & 16.55 & 6.76 & 6.04 & 7.51 & & 21.88 & 13.51 & 31.25 & & 0.10 & -26.19 & 23.36 & 039 \\
\hline 35.08 & 17.33 & 6.83 & 6.25 & 7.52 & & 21.04 & 12.02 & 31.69 & 25 & -2.19 & -38.57 & 24.82 & 248 \\
\hline 35.21 & 2.67 & 7.42 & 6.97 & 7.91 & & 3.56 & 0.08 & 11.58 & 606 & 9.55 & -87.96 & 89.86 & 2.08131 \\
\hline 35.21 & 2.67 & 7.34 & 6.92 & 7.80 & & 5.38 & 0.20 & 11.68 & 621 & -20.89 & -89.09 & 89.71 & 1.53891 \\
\hline 35.35 & 2.67 & 7.35 & 6.84 & 7.75 & & 3.74 & 0.30 & 10.80 & 063 & 10.93 & -89.26 & 89.81 & 2.07634 \\
\hline 35.35 & 12.67 & 7.29 & 6.75 & 7.72 & & 6.04 & 0.40 & 10.81 & 987 & -18.80 & -87.29 & 89.93 & 1.45497 \\
\hline 35.49 & 2.67 & 7.39 & 6.92 & 7.83 & 93 & 3.72 & 0.22 & 9.65 & 7775 & 9.12 & -88.62 & 89.53 & 2.00150 \\
\hline 35.49 & 12.67 & 7.29 & 6.80 & 7.70 & & 5.56 & 0.32 & 11.13 & 220 & 20.15 & -89.72 & 86.71 & 1.73098 \\
\hline 35.62 & 2.67 & 7.34 & 6.94 & 7.73 & 0.00655 & 3.48 & 0.17 & 11.21 & 0.08051 & 6.46 & -89.36 & 89.65 & 2.17972 \\
\hline 35.62 & .67 & 7.26 & 6.84 & 7.83 & 25 & 5.84 & 0.39 & 12.44 & 08 & -19.60 & -88.28 & 85.64 & 1.5 \\
\hline 35.76 & 2.67 & 7.38 & 6.95 & 7.78 & 0.00633 & 3.98 & 0.09 & 9.94 & 0.08809 & 12.88 & -87.16 & 89.24 & 1.97899 \\
\hline 35.76 & 12.67 & 7.29 & 6.87 & 7.77 & 0.00688 & 6.04 & 0.47 & 11.65 & 0.10441 & -18.46 & -87.35 & 87.68 & 1.35033 \\
\hline 35.89 & 2.67 & 7.40 & 6.89 & 7.94 & & 3.80 & 0.11 & 14.32 & 274 & 9.35 & -89.98 & 89.98 & 0.74534 \\
\hline 35.89 & 3.45 & 7.33 & 6.92 & 7.84 & 0.00460 & 4.42 & 0.22 & 15.67 & 0.07255 & 6.44 & -89.23 & 89.88 & 1.48137 \\
\hline 35.89 & 4.22 & 7.31 & 6.85 & 7.74 & 0.00464 & 5.29 & 0.36 & 13.79 & 0.07424 & 18.95 & -88.88 & 89.60 & 1.25498 \\
\hline
\end{tabular}


Water Velocity Measurements on an ESBS at John Day Dam

\begin{tabular}{|c|c|c|c|c|c|c|c|c|c|c|c|c|c|}
\hline 35.89 & 5.00 & 7.32 & 6.82 & 8.01 & 0.00458 & 4.83 & 0.32 & 13.88 & 0.06968 & 17.18 & -89.98 & 89.91 & 1.37183 \\
\hline 35.89 & 5.78 & 7.29 & 6.74 & 7.89 & 0.00505 & 4.28 & 0.23 & 13.87 & 0.07196 & 7.13 & -89.85 & 89.81 & 1.43144 \\
\hline 35.89 & 6.55 & 7.27 & 6.80 & 7.72 & 0.00501 & 4.36 & 0.08 & 12.62 & 07233 & 2.08 & -89.95 & \begin{tabular}{|l|}
89.47 \\
\end{tabular} & .42216 \\
\hline 35.89 & 7.33 & 7.25 & 6.71 & 7.77 & 0.00294 & 3.98 & 0.12 & 13.99 & 0.03877 & -0.88 & -89.87 & 89.92 & 0.79675 \\
\hline 35.89 & 2.67 & 7.33 & 6.76 & 7.94 & 0.00247 & 5.71 & 0.01 & 15.51 & 0.0 & 17.74 & -89.91 & 89.98 & 0.56146 \\
\hline 35.89 & 3.45 & 7.31 & 6.77 & 7.85 & 0.0 & 5.67 & 0.25 & 14.01 & 69 & -19.12 & -89.92 & 89.93 & 1.18712 \\
\hline 35.89 & 14.22 & 7.37 & 6.83 & 7.82 & 0.0 & 4.87 & 0.05 & 13.11 & 0.0 & -20.15 & -89.97 & 89.85 & 1.42398 \\
\hline 35.89 & 5.00 & 7.45 & 6.84 & 7.96 & 0.0 & 4.80 & 0.06 & 12.01 & 0.0 & -20.99 & -89.94 & 89.98 & 1.57221 \\
\hline 35.89 & 15.78 & 7.50 & 6.98 & 7.97 & 0.0 & 6.13 & 0.09 & 14.61 & 0.0 & -33.64 & -89.47 & 89.80 & 1.24823 \\
\hline 35.89 & 16.55 & 7.62 & 7.10 & 8.15 & 0.00516 & 7.30 & 0.31 & 16.44 & 0.08163 & -35.64 & -89.88 & 89.06 & 0.93103 \\
\hline 35.89 & 17.33 & 7.72 & 7.03 & 8.37 & 0.00298 & 7.41 & 0.29 & 16.52 & 0.04904 & -37.55 & -89.85 & 89.99 & 0.53090 \\
\hline
\end{tabular}




\section{Appendix C}

\section{Turbulence Intensity Values at 155 MW Turbine Load}




\section{Appendix C}

\section{Turbulence Intensity Values at 155 MW Turbine Load}

The table in this appendix lists turbulence intensity values for $\mathrm{X}, \mathrm{Y}$, and $\mathrm{Z}$ vectors and magnitude turbulence intensity for flow data collected at locations along the bar screen surface of the ESBS, at 155 MW turbine load.

\begin{tabular}{|c|c|c|c|c|c|}
\hline \multicolumn{2}{|c|}{ Location (ft.) } & \multicolumn{4}{|c|}{ RMS Turbulence Intensity (ft/sec) } \\
\hline Vertical & Horizontal & Magnitude & X-vector & Y-vector & Z-vector \\
\hline-0.02 & 2.67 & 0.17 & 0.23 & 0.25 & 0.27 \\
\hline-0.02 & 3.45 & 0.24 & 0.31 & 0.43 & 0.55 \\
\hline-0.02 & 4.22 & 0.25 & 0.42 & 0.63 & 0.35 \\
\hline-0.02 & 5.00 & 0.20 & 0.34 & 0.42 & 0.25 \\
\hline-0.02 & 5.78 & 0.36 & 0.39 & 0.50 & 0.54 \\
\hline-0.02 & 6.55 & 0.33 & 0.28 & 0.31 & 0.54 \\
\hline-0.02 & 7.33 & 0.15 & 0.25 & 0.22 & 0.22 \\
\hline-0.02 & 12.67 & 0.17 & 0.27 & 0.23 & 0.26 \\
\hline-0.02 & 13.45 & 0.34 & 0.39 & 0.30 & 0.42 \\
\hline-0.02 & 14.22 & 0.60 & 0.44 & 0.43 & 0.61 \\
\hline-0.02 & 15.00 & 0.36 & 0.27 & 0.34 & 0.43 \\
\hline-0.02 & 15.78 & 0.50 & 0.41 & 0.51 & 0.68 \\
\hline-0.02 & 16.55 & 0.24 & 0.30 & 0.28 & 0.37 \\
\hline-0.02 & 17.33 & 0.23 & 0.38 & 0.35 & 0.35 \\
\hline 0.30 & 7.33 & 0.66 & 0.40 & 0.19 & 0.71 \\
\hline 0.30 & 17.33 & 0.84 & 0.74 & 0.28 & 0.61 \\
\hline 0.63 & 7.33 & 0.34 & 0.27 & 0.17 & 0.37 \\
\hline 0.63 & 17.33 & 0.38 & 0.42 & 0.24 & 0.46 \\
\hline 0.95 & 7.33 & 0.14 & 0.15 & 0.14 & 0.18 \\
\hline 0.95 & 17.33 & 0.22 & 0.22 & 0.24 & 0.17 \\
\hline 1.27 & 7.33 & 0.19 & 0.22 & 0.16 & 0.20 \\
\hline 1.27 & 17.33 & 0.17 & 0.17 & 0.26 & 0.24 \\
\hline 1.60 & 7.33 & 0.15 & 0.17 & 0.19 & 0.14 \\
\hline 1.60 & 17.33 & 0.21 & 0.22 & 0.23 & 0.22 \\
\hline 1.92 & 2.67 & 0.16 & 0.18 & 0.23 & 0.19 \\
\hline 1.92 & 3.45 & 0.16 & 0.21 & 0.18 & 0.16 \\
\hline 1.92 & 4.22 & 0.17 & 0.22 & 0.21 & 0.19 \\
\hline 1.92 & 5.00 & 0.21 & 0.22 & 0.20 & 0.19 \\
\hline 1.92 & 5.78 & 0.18 & 0.20 & 0.29 & 0.17 \\
\hline 1.92 & 6.55 & 0.19 & 0.21 & 0.15 & 0.17 \\
\hline 1.92 & 7.33 & 0.16 & 0.18 & 0.18 & 0.18 \\
\hline 1.92 & 12.67 & 0.18 & 0.22 & 0.22 & 0.22 \\
\hline 1.92 & 13.45 & 0.17 & 0.20 & 0.21 & 0.19 \\
\hline 1.92 & 14.22 & 0.17 & 0.19 & 0.20 & 0.18 \\
\hline 1.92 & 15.00 & 0.19 & 0.22 & 0.28 & 0.17 \\
\hline 1.92 & 15.78 & 0.15 & 0.17 & 0.19 & 0.18 \\
\hline 1.92 & 16.55 & 0.17 & 0.22 & 0.24 & 0.16 \\
\hline 1.92 & 17.33 & 0.23 & 0.25 & 0.25 & 0.22 \\
\hline 2.67 & 2.67 & 0.59 & 0.50 & 0.19 & 0.42 \\
\hline 2.67 & 12.67 & 0.48 & 0.42 & 0.20 & 0.39 \\
\hline 3.42 & 2.67 & 0.29 & 0.28 & 0.20 & 0.25 \\
\hline 3.42 & 12.67 & 0.28 & 0.27 & 0.23 & 0.23 \\
\hline 4.17 & 2.67 & 0.17 & 0.22 & 0.20 & 0.20 \\
\hline 4.17 & 12.67 & 0.18 & 0.21 & 0.20 & 0.21 \\
\hline 4.92 & 2.67 & 0.27 & 0.25 & 0.19 & 0.26 \\
\hline
\end{tabular}


Water Velocity Measurements on an ESBS at John Day Dam

\begin{tabular}{|c|c|c|c|c|c|}
\hline 4.92 & 12.67 & 0.30 & 0.29 & 0.21 & 0.23 \\
\hline 5.67 & 2.67 & 0.43 & 0.34 & 0.18 & 0.75 \\
\hline 5.67 & 12.67 & 0.35 & 0.31 & 0.18 & 0.61 \\
\hline 6.41 & 2.67 & 0.42 & 0.42 & 0.37 & 0.29 \\
\hline 6.41 & 3.45 & 0.35 & 0.35 & 0.35 & 0.29 \\
\hline 6.41 & 4.22 & 0.33 & 0.34 & 0.36 & 0.27 \\
\hline 6.41 & 5.00 & 0.33 & 0.32 & 0.34 & 0.29 \\
\hline 6.41 & 5.78 & 0.33 & 0.33 & 0.33 & 0.29 \\
\hline 6.41 & 6.55 & 0.38 & 0.37 & 0.34 & 0.35 \\
\hline 6.41 & 7.33 & 0.37 & 0.36 & 0.34 & 0.30 \\
\hline 6.41 & 12.67 & 0.32 & 0.31 & 0.35 & 0.26 \\
\hline 6.41 & 13.45 & 0.33 & 0.33 & 0.33 & 0.28 \\
\hline 6.41 & 14.22 & 0.33 & 0.33 & 0.33 & 0.26 \\
\hline 6.41 & 15.00 & 0.33 & 0.34 & 0.33 & 0.25 \\
\hline 6.41 & 15.78 & 0.33 & 0.33 & 0.33 & 0.22 \\
\hline 6.41 & 16.55 & 0.30 & 0.30 & 0.31 & 0.23 \\
\hline 6.41 & 17.33 & 0.42 & 0.38 & 0.36 & 0.33 \\
\hline 6.83 & 7.33 & 0.38 & 0.37 & 0.33 & 0.32 \\
\hline 6.83 & 17.33 & 0.43 & 0.40 & 0.36 & 0.34 \\
\hline 7.25 & 7.33 & 0.42 & 0.40 & 0.33 & 0.31 \\
\hline 7.25 & 17.33 & 0.43 & 0.41 & 0.37 & 0.31 \\
\hline 7.66 & 7.33 & 0.38 & 0.37 & 0.33 & 0.27 \\
\hline 7.66 & 17.33 & 0.46 & 0.42 & 0.36 & 0.33 \\
\hline 8.08 & 7.33 & 0.35 & 0.34 & 0.34 & 0.32 \\
\hline 8.08 & 17.33 & 0.40 & 0.38 & 0.36 & 0.32 \\
\hline 8.50 & 7.33 & 0.36 & 0.36 & 0.33 & 0.26 \\
\hline 8.50 & 17.33 & 0.50 & 0.45 & 0.38 & 0.37 \\
\hline 8.85 & 2.67 & 0.37 & 0.36 & 0.32 & 0.26 \\
\hline 8.85 & 3.45 & 0.34 & 0.33 & 0.36 & 0.25 \\
\hline 8.85 & 4.22 & 0.32 & 0.32 & 0.31 & 0.26 \\
\hline 8.85 & 5.00 & 0.34 & 0.34 & 0.34 & 0.25 \\
\hline 8.85 & 5.78 & 0.33 & 0.33 & 0.32 & 0.30 \\
\hline 8.85 & 6.55 & 0.33 & 0.32 & 0.32 & 0.29 \\
\hline 8.85 & 7.33 & 0.37 & 0.36 & 0.31 & 0.27 \\
\hline 8.85 & 12.67 & 0.32 & 0.32 & 0.31 & 0.23 \\
\hline 8.85 & 13.45 & 0.34 & 0.33 & 0.33 & 0.23 \\
\hline 8.85 & 14.22 & 0.36 & 0.38 & 0.33 & 0.22 \\
\hline 8.85 & 15.00 & 0.33 & 0.33 & 0.29 & 0.24 \\
\hline 8.85 & 15.78 & 0.33 & 0.31 & 0.33 & 0.25 \\
\hline 8.85 & 16.55 & 0.41 & 0.38 & 0.35 & 0.32 \\
\hline 8.85 & 17.33 & 0.40 & 0.36 & 0.35 & 0.30 \\
\hline 8.92 & 2.67 & 0.37 & 0.36 & 0.34 & 0.26 \\
\hline 8.92 & 3.45 & 0.34 & 0.34 & 0.33 & 0.26 \\
\hline 8.92 & 4.22 & 0.34 & 0.33 & 0.34 & 0.25 \\
\hline 8.92 & 5.00 & 0.34 & 0.35 & 0.32 & 0.25 \\
\hline 8.92 & 5.78 & 0.35 & 0.35 & 0.32 & 0.26 \\
\hline 8.92 & 6.55 & 0.32 & 0.32 & 0.33 & 0.29 \\
\hline 8.92 & 7.33 & 0.35 & 0.34 & 0.33 & 0.26 \\
\hline 8.92 & 12.67 & 0.31 & 0.31 & 0.33 & 0.23 \\
\hline 8.92 & 13.45 & 0.31 & 0.31 & 0.35 & 0.23 \\
\hline 8.92 & 14.22 & 0.32 & 0.32 & 0.34 & 0.22 \\
\hline 8.92 & 15.00 & 0.33 & 0.33 & 0.37 & 0.24 \\
\hline 8.92 & 15.78 & 0.33 & 0.32 & 0.32 & 0.22 \\
\hline 8.92 & 16.55 & 0.41 & 0.39 & 0.36 & 0.31 \\
\hline 8.92 & 17.33 & 0.43 & 0.40 & 0.35 & 0.32 \\
\hline 8.94 & 2.67 & 0.34 & 0.31 & 0.36 & 0.26 \\
\hline 8.94 & 12.67 & 0.34 & 0.33 & 0.28 & 0.19 \\
\hline 9.02 & 2.67 & 0.31 & 0.29 & 0.35 & 0.27 \\
\hline 9.02 & 12.67 & 0.32 & 0.33 & 0.31 & 0.20 \\
\hline
\end{tabular}


Water Velocity Measurements on an ESBS at John Day Dam

\begin{tabular}{|c|c|c|c|c|c|}
\hline 9.10 & 2.67 & 0.43 & 0.42 & 0.35 & 0.28 \\
\hline 9.10 & 12.67 & 0.26 & 0.26 & 0.29 & 0.28 \\
\hline 9.19 & 2.67 & 0.29 & 0.27 & 0.31 & 0.24 \\
\hline 9.19 & 12.67 & 0.27 & 0.26 & 0.36 & 0.22 \\
\hline 9.27 & 2.67 & 0.35 & 0.33 & 0.32 & 0.31 \\
\hline 9.27 & 12.67 & 0.35 & 0.35 & 0.30 & 0.19 \\
\hline 9.35 & 2.67 & 0.37 & 0.36 & 0.31 & 0.26 \\
\hline 9.35 & 3.45 & 0.30 & 0.30 & 0.30 & 0.26 \\
\hline 9.35 & 4.22 & 0.33 & 0.33 & 0.36 & 0.25 \\
\hline 9.35 & 5.00 & 0.30 & 0.31 & 0.31 & 0.22 \\
\hline 9.35 & 5.78 & 0.38 & 0.38 & 0.34 & 0.25 \\
\hline 9.35 & 6.55 & 0.32 & 0.32 & 0.30 & 0.28 \\
\hline 9.35 & 7.33 & 0.36 & 0.35 & 0.32 & 0.26 \\
\hline 9.35 & 12.67 & 0.33 & 0.33 & 0.32 & 0.22 \\
\hline 9.35 & 13.45 & 0.31 & 0.31 & 0.31 & 0.22 \\
\hline 9.35 & 14.22 & 0.31 & 0.32 & 0.33 & 0.21 \\
\hline 9.35 & 15.00 & 0.32 & 0.31 & 0.35 & 0.21 \\
\hline 9.35 & 15.78 & 0.32 & 0.32 & 0.34 & 0.23 \\
\hline 9.35 & 16.55 & 0.38 & 0.35 & 0.34 & 0.32 \\
\hline 9.35 & 17.33 & 0.41 & 0.38 & 0.36 & 0.32 \\
\hline 9.38 & 2.67 & 0.39 & 0.38 & 0.34 & 0.24 \\
\hline 9.38 & 12.67 & 0.32 & 0.31 & 0.32 & 0.20 \\
\hline 9.44 & 7.33 & 0.31 & 0.30 & 0.33 & 0.21 \\
\hline 9.44 & 17.33 & 0.40 & 0.38 & 0.34 & 0.27 \\
\hline 9.52 & 7.33 & 0.35 & 0.35 & 0.30 & 0.12 \\
\hline 9.52 & 17.33 & 0.33 & 0.35 & 0.34 & 0.24 \\
\hline 9.60 & 7.33 & 0.34 & 0.31 & 0.32 & 0.30 \\
\hline 9.60 & 17.33 & 0.38 & 0.36 & 0.32 & 0.30 \\
\hline 9.69 & 7.33 & 0.32 & 0.32 & 0.34 & 0.19 \\
\hline 9.69 & 17.33 & 0.45 & 0.45 & 0.32 & 0.21 \\
\hline 9.77 & 7.33 & 0.34 & 0.33 & 0.32 & 0.25 \\
\hline 9.77 & 17.33 & 0.41 & 0.38 & 0.29 & 0.28 \\
\hline 9.84 & 2.67 & 0.37 & 0.37 & 0.33 & 0.21 \\
\hline 9.84 & 12.67 & 0.32 & 0.32 & 0.32 & 0.19 \\
\hline 9.85 & 2.67 & 0.39 & 0.38 & 0.32 & 0.23 \\
\hline 9.85 & 3.45 & 0.34 & 0.34 & 0.31 & 0.25 \\
\hline 9.85 & 4.22 & 0.34 & 0.34 & 0.33 & 0.21 \\
\hline 9.85 & 5.00 & 0.36 & 0.36 & 0.33 & 0.21 \\
\hline 9.85 & 5.78 & 0.32 & 0.32 & 0.37 & 0.22 \\
\hline 9.85 & 6.55 & 0.34 & 0.34 & 0.30 & 0.27 \\
\hline 9.85 & 7.33 & 0.34 & 0.33 & 0.34 & 0.22 \\
\hline 9.85 & 12.67 & 0.32 & 0.31 & 0.30 & 0.19 \\
\hline 9.85 & 13.45 & 0.32 & 0.32 & 0.31 & 0.21 \\
\hline 9.85 & 14.22 & 0.31 & 0.31 & 0.29 & 0.22 \\
\hline 9.85 & 15.00 & 0.33 & 0.31 & 0.34 & 0.22 \\
\hline 9.85 & 15.78 & 0.34 & 0.33 & 0.36 & 0.23 \\
\hline 9.85 & 16.55 & 0.38 & 0.36 & 0.33 & 0.28 \\
\hline 9.85 & 17.33 & 0.41 & 0.38 & 0.35 & 0.31 \\
\hline 9.93 & 2.67 & 0.39 & 0.38 & 0.29 & 0.26 \\
\hline 9.93 & 12.67 & 0.29 & 0.28 & 0.29 & 0.17 \\
\hline 10.00 & 2.67 & 0.34 & 0.35 & 0.23 & 0.28 \\
\hline 10.00 & 12.67 & 0.37 & 0.33 & 0.27 & 0.31 \\
\hline 10.07 & 2.67 & 0.43 & 0.41 & 0.31 & 0.26 \\
\hline 10.07 & 12.67 & 0.31 & 0.30 & 0.32 & 0.23 \\
\hline 10.13 & 2.67 & 0.36 & 0.35 & 0.43 & 0.23 \\
\hline 10.13 & 12.67 & 0.34 & 0.33 & 0.30 & 0.18 \\
\hline 10.20 & 2.67 & 0.33 & 0.32 & 0.33 & 0.25 \\
\hline 10.20 & 12.67 & 0.39 & 0.35 & 0.35 & 0.23 \\
\hline 10.27 & 2.67 & 0.39 & 0.38 & 0.34 & 0.25 \\
\hline
\end{tabular}


Water Velocity Measurements on an ESBS at John Day Dam

\begin{tabular}{|c|c|c|c|c|c|}
\hline 10.27 & 3.45 & 0.38 & 0.36 & 0.33 & 0.25 \\
\hline 10.27 & 4.22 & 0.40 & 0.39 & 0.36 & 0.26 \\
\hline 10.27 & 5.00 & 0.33 & 0.34 & 0.30 & 0.21 \\
\hline 10.27 & 5.78 & 0.34 & 0.34 & 0.32 & 0.21 \\
\hline 10.27 & 6.55 & 0.36 & 0.34 & 0.33 & 0.28 \\
\hline 10.27 & 7.33 & 0.37 & 0.36 & 0.33 & 0.25 \\
\hline 10.27 & 12.67 & 0.34 & 0.32 & 0.32 & 0.22 \\
\hline 10.27 & 13.45 & 0.33 & 0.33 & 0.31 & 0.22 \\
\hline 10.27 & 14.22 & 0.37 & 0.36 & 0.31 & 0.24 \\
\hline 10.27 & 15.00 & 0.33 & 0.33 & 0.30 & 0.22 \\
\hline 10.27 & 15.78 & 0.34 & 0.34 & 0.33 & 0.23 \\
\hline 10.27 & 16.55 & 0.39 & \begin{tabular}{l|l|}
0.37 \\
\end{tabular} & 0.34 & 0.27 \\
\hline 10.27 & 17.33 & 0.38 & 0.36 & 0.33 & 0.29 \\
\hline 10.29 & 2.67 & 0.54 & 0.51 & 0.31 & 0.31 \\
\hline 10.29 & 12.67 & 0.44 & 0.42 & 0.31 & 0.30 \\
\hline 10.75 & 2.67 & 0.38 & 0.38 & 0.33 & 0.23 \\
\hline 10.75 & 12.67 & 0.32 & 0.31 & 0.32 & 0.25 \\
\hline 10.86 & 2.67 & 0.39 & 0.38 & 0.34 & 0.25 \\
\hline 10.86 & 3.45 & 0.35 & 0.34 & 0.34 & 0.27 \\
\hline 10.86 & 4.22 & 0.36 & 0.36 & 0.31 & 0.25 \\
\hline 10.86 & 5.00 & 0.36 & 0.36 & 0.31 & 0.22 \\
\hline 10.86 & 5.78 & 0.34 & 0.33 & 0.31 & 0.28 \\
\hline 10.86 & 6.55 & 0.33 & 0.32 & 0.31 & 0.26 \\
\hline 10.86 & 7.33 & 0.39 & 0.37 & 0.30 & 0.29 \\
\hline 10.86 & 12.67 & 0.34 & 0.33 & 0.32 & 0.24 \\
\hline 10.86 & 13.45 & 0.35 & 0.34 & 0.30 & 0.23 \\
\hline 10.86 & 14.22 & 0.35 & 0.34 & 0.30 & 0.26 \\
\hline 10.86 & 15.00 & 0.39 & 0.39 & 0.33 & 0.24 \\
\hline 10.86 & 15.78 & 0.36 & 0.34 & 0.31 & 0.22 \\
\hline 10.86 & 16.55 & 0.36 & 0.35 & 0.35 & 0.27 \\
\hline 10.86 & 17.33 & 0.39 & 0.36 & 0.37 & 0.30 \\
\hline 10.92 & 2.67 & 0.28 & 0.27 & 0.24 & 0.22 \\
\hline 10.92 & 12.67 & 0.53 & 0.51 & 0.40 & 0.25 \\
\hline 10.97 & 2.67 & 0.42 & 0.41 & 0.37 & 0.28 \\
\hline 10.97 & 12.67 & 0.26 & 0.25 & 0.27 & 0.20 \\
\hline 11.03 & 2.67 & 0.36 & 0.34 & 0.24 & 0.32 \\
\hline 11.03 & 12.67 & 0.30 & 0.32 & 0.32 & 0.21 \\
\hline 11.09 & 2.67 & 0.35 & 0.32 & 0.32 & 0.30 \\
\hline 11.09 & 12.67 & 0.30 & 0.30 & 0.36 & 0.16 \\
\hline 11.14 & 2.67 & 0.36 & 0.29 & 0.36 & 0.29 \\
\hline 11.14 & 12.67 & 0.25 & 0.25 & 0.30 & 0.23 \\
\hline 11.20 & 2.67 & 0.36 & 0.35 & 0.31 & 0.25 \\
\hline 11.20 & 3.45 & 0.36 & 0.34 & 0.32 & 0.27 \\
\hline 11.20 & 4.22 & 0.33 & 0.33 & 0.31 & 0.24 \\
\hline 11.20 & 5.00 & 0.37 & 0.37 & 0.32 & 0.22 \\
\hline 11.20 & 5.78 & 0.38 & 0.37 & 0.28 & 0.25 \\
\hline 11.20 & 6.55 & 0.33 & 0.32 & 0.33 & 0.25 \\
\hline 11.20 & 7.33 & 0.33 & 0.32 & 0.27 & 0.22 \\
\hline 11.20 & 12.67 & 0.35 & 0.34 & 0.30 & 0.22 \\
\hline 11.20 & 13.45 & 0.35 & 0.34 & 0.29 & 0.24 \\
\hline 11.20 & 14.22 & 0.32 & 0.31 & 0.29 & 0.20 \\
\hline 11.20 & 15.00 & 0.36 & 0.36 & 0.32 & 0.20 \\
\hline 11.20 & 15.78 & 0.36 & 0.35 & 0.33 & 0.23 \\
\hline 11.20 & 16.55 & 0.36 & 0.34 & 0.29 & 0.25 \\
\hline 11.20 & 17.33 & 0.34 & 0.32 & 0.30 & 0.26 \\
\hline 11.21 & 2.67 & 0.36 & 0.36 & 0.29 & 0.22 \\
\hline 11.21 & 12.67 & 0.34 & 0.33 & 0.31 & 0.24 \\
\hline 11.28 & 7.33 & 0.23 & 0.24 & 0.32 & 0.19 \\
\hline 11.28 & 17.33 & 0.24 & 0.22 & 0.22 & 0.19 \\
\hline
\end{tabular}


Water Velocity Measurements on an ESBS at John Day Dam

\begin{tabular}{|c|c|c|c|c|c|}
\hline 11.37 & 7.33 & 0.39 & 0.37 & 0.34 & 0.21 \\
\hline 11.37 & 17.33 & 0.30 & 0.29 & 0.27 & 0.23 \\
\hline 11.45 & 7.33 & 0.22 & 0.24 & 0.38 & 0.20 \\
\hline 11.45 & 17.33 & 0.27 & 0.26 & 0.28 & 0.24 \\
\hline 11.53 & 7.33 & 0.34 & 0.31 & 0.21 & 0.32 \\
\hline 11.53 & 17.33 & 0.32 & 0.28 & 0.23 & 0.24 \\
\hline 11.62 & 7.33 & 0.28 & 0.27 & 0.26 & 0.29 \\
\hline 11.62 & 17.33 & 0.33 & 0.28 & 0.29 & 0.31 \\
\hline 11.67 & 2.67 & 0.35 & 0.35 & 0.30 & 0.23 \\
\hline 11.67 & 3.45 & 0.36 & 0.36 & 0.31 & 0.25 \\
\hline 11.67 & 4.22 & 0.41 & 0.40 & 0.32 & 0.24 \\
\hline 11.67 & 5.00 & 0.38 & 0.37 & 0.33 & 0.24 \\
\hline 11.67 & 5.78 & 0.37 & 0.36 & 0.33 & 0.25 \\
\hline 11.67 & 6.55 & 0.39 & 0.37 & 0.34 & 0.27 \\
\hline 11.67 & 7.33 & 0.37 & 0.35 & 0.31 & 0.26 \\
\hline 11.67 & 12.67 & 0.35 & 0.34 & 0.32 & 0.23 \\
\hline 11.67 & 13.45 & 0.37 & 0.36 & 0.32 & 0.24 \\
\hline 11.67 & 14.22 & 0.38 & 0.37 & 0.29 & 0.22 \\
\hline 11.67 & 15.00 & 0.36 & 0.36 & 0.31 & 0.21 \\
\hline 11.67 & 15.78 & 0.37 & 0.36 & 0.33 & 0.25 \\
\hline 11.67 & 16.55 & 0.36 & 0.34 & 0.31 & 0.28 \\
\hline 11.67 & 17.33 & 0.38 & 0.36 & 0.33 & 0.27 \\
\hline 11.70 & 2.67 & 0.37 & 0.36 & 0.31 & 0.25 \\
\hline 11.70 & 3.45 & 0.41 & 0.39 & 0.30 & 0.29 \\
\hline 11.70 & 4.22 & 0.40 & 0.39 & 0.31 & 0.27 \\
\hline 11.70 & 5.00 & 0.40 & 0.40 & 0.33 & 0.22 \\
\hline 11.70 & 5.78 & 0.37 & 0.37 & 0.32 & 0.24 \\
\hline 11.70 & 6.55 & 0.33 & 0.32 & 0.30 & 0.23 \\
\hline 11.70 & 7.33 & 0.37 & 0.36 & 0.32 & 0.28 \\
\hline 11.70 & 12.67 & 0.36 & 0.34 & 0.32 & 0.23 \\
\hline 11.70 & 13.45 & 0.37 & 0.36 & 0.29 & 0.26 \\
\hline 11.70 & 14.22 & 0.36 & 0.36 & 0.29 & 0.23 \\
\hline 11.70 & 15.00 & 0.34 & 0.34 & 0.31 & 0.22 \\
\hline 11.70 & 15.78 & 0.36 & 0.33 & 0.33 & 0.26 \\
\hline 11.70 & 16.55 & 0.36 & 0.33 & 0.34 & 0.24 \\
\hline 11.70 & 17.33 & 0.37 & 0.35 & 0.31 & 0.24 \\
\hline 12.09 & 7.33 & 0.39 & 0.35 & 0.30 & 0.29 \\
\hline 12.09 & 17.33 & 0.41 & 0.39 & 0.33 & 0.25 \\
\hline 12.50 & 7.33 & 0.33 & 0.32 & 0.30 & 0.26 \\
\hline 12.50 & 17.33 & 0.39 & 0.34 & 0.32 & 0.27 \\
\hline 12.92 & 7.33 & 0.32 & 0.32 & 0.29 & 0.24 \\
\hline 12.92 & 17.33 & 0.36 & 0.33 & 0.34 & 0.24 \\
\hline 13.34 & 7.33 & 0.33 & 0.32 & 0.29 & 0.24 \\
\hline 13.34 & 17.33 & 0.34 & 0.29 & 0.36 & 0.27 \\
\hline 13.75 & 7.33 & 0.40 & 0.38 & 0.30 & 0.26 \\
\hline 13.75 & 17.33 & 0.36 & 0.34 & 0.31 & 0.22 \\
\hline 14.22 & 2.67 & 0.37 & 0.36 & 0.30 & 0.24 \\
\hline 14.22 & 3.45 & 0.35 & 0.33 & 0.32 & 0.24 \\
\hline 14.22 & 4.22 & 0.35 & 0.34 & 0.31 & 0.22 \\
\hline 14.22 & 5.00 & 0.37 & 0.36 & 0.32 & 0.22 \\
\hline 14.22 & 5.78 & 0.37 & 0.36 & 0.32 & 0.21 \\
\hline 14.22 & 6.55 & 0.35 & 0.34 & 0.30 & 0.23 \\
\hline 14.22 & 7.33 & 0.35 & 0.34 & 0.30 & 0.23 \\
\hline 14.22 & 12.67 & 0.32 & 0.30 & 0.29 & 0.21 \\
\hline 14.22 & 13.45 & 0.30 & 0.29 & 0.28 & 0.19 \\
\hline 14.22 & 14.22 & 0.35 & 0.33 & 0.30 & 0.20 \\
\hline 14.22 & 15.00 & 0.41 & 0.37 & 0.32 & 0.28 \\
\hline 14.22 & 15.78 & 0.37 & 0.33 & 0.33 & 0.27 \\
\hline 14.22 & 16.55 & 0.39 & 0.35 & 0.36 & 0.25 \\
\hline
\end{tabular}


Water Velocity Measurements on an ESBS at John Day Dam

\begin{tabular}{|c|c|c|c|c|c|}
\hline 14.22 & 17.33 & 0.35 & 0.33 & 0.33 & 0.24 \\
\hline 14.68 & 2.67 & 0.36 & 0.36 & 0.28 & 0.23 \\
\hline 14.68 & 12.67 & 0.32 & 0.31 & 0.28 & 0.22 \\
\hline 15.15 & 2.67 & 0.31 & 0.31 & 0.28 & 0.22 \\
\hline 15.15 & 12.67 & 0.31 & 0.31 & 0.28 & 0.19 \\
\hline 15.61 & 2.67 & 0.32 & 0.33 & 0.30 & 0.21 \\
\hline 15.61 & 12.67 & 0.31 & 0.30 & 0.29 & 0.21 \\
\hline 16.07 & 2.67 & 0.30 & 0.30 & 0.28 & 0.23 \\
\hline 16.07 & 12.67 & 0.28 & 0.27 & 0.24 & 0.18 \\
\hline 16.54 & 2.67 & 0.34 & 0.33 & 0.27 & 0.20 \\
\hline 16.54 & 12.67 & 0.30 & 0.30 & 0.27 & 0.19 \\
\hline 16.99 & 2.67 & 0.33 & 0.32 & 0.27 & 0.21 \\
\hline 16.99 & 3.45 & 0.34 & 0.34 & 0.29 & 0.22 \\
\hline 16.99 & 4.22 & 0.31 & 0.30 & 0.27 & 0.20 \\
\hline 16.99 & 5.00 & 0.34 & 0.33 & 0.30 & 0.19 \\
\hline 16.99 & 5.78 & 0.39 & 0.38 & 0.29 & 0.21 \\
\hline 16.99 & 6.55 & 0.34 & 0.33 & 0.28 & 0.19 \\
\hline 16.99 & 7.33 & 0.32 & 0.32 & 0.26 & 0.21 \\
\hline 16.99 & 12.67 & 0.29 & 0.28 & 0.26 & 0.19 \\
\hline 16.99 & 13.45 & 0.31 & 0.29 & 0.28 & 0.19 \\
\hline 16.99 & 14.22 & 0.31 & 0.32 & 0.28 & 0.19 \\
\hline 16.99 & 15.00 & 0.30 & 0.30 & 0.29 & 0.21 \\
\hline 16.99 & 15.78 & 0.31 & 0.30 & 0.29 & 0.23 \\
\hline 16.99 & 16.55 & 0.32 & 0.32 & 0.31 & 0.25 \\
\hline 16.99 & 17.33 & 0.32 & 0.32 & 0.32 & 0.26 \\
\hline 17.41 & 7.33 & 0.33 & 0.35 & 0.29 & 0.18 \\
\hline 17.41 & 17.33 & 0.34 & 0.31 & 0.30 & 0.29 \\
\hline 17.83 & 7.33 & 0.27 & 0.28 & 0.26 & 0.18 \\
\hline 17.83 & 17.33 & 0.28 & 0.31 & 0.29 & 0.23 \\
\hline 18.24 & 7.33 & 0.33 & 0.33 & 0.27 & 0.20 \\
\hline 18.24 & 17.33 & 0.28 & 0.32 & 0.30 & 0.28 \\
\hline 18.66 & 7.33 & 0.23 & 0.25 & 0.29 & 0.20 \\
\hline 18.66 & 17.33 & 0.26 & 0.31 & 0.31 & 0.25 \\
\hline 19.08 & 7.33 & 0.34 & 0.36 & 0.25 & 0.19 \\
\hline 19.08 & 17.33 & 0.39 & 0.38 & 0.29 & 0.23 \\
\hline 19.47 & 2.67 & 0.33 & 0.33 & 0.27 & 0.22 \\
\hline 19.47 & 3.45 & 0.33 & 0.32 & 0.27 & 0.20 \\
\hline 19.47 & 4.22 & 0.32 & 0.31 & 0.26 & 0.19 \\
\hline 19.47 & 5.00 & 0.34 & 0.35 & 0.28 & 0.19 \\
\hline 19.47 & 5.78 & 0.35 & 0.35 & 0.25 & 0.20 \\
\hline 19.47 & 6.55 & 0.30 & 0.30 & 0.26 & 0.20 \\
\hline 19.47 & 7.33 & 0.31 & 0.31 & 0.26 & 0.20 \\
\hline 19.47 & 12.67 & 0.29 & 0.28 & 0.26 & 0.18 \\
\hline 19.47 & 13.45 & 0.31 & 0.31 & 0.26 & 0.16 \\
\hline 19.47 & 14.22 & 0.31 & 0.32 & 0.28 & 0.18 \\
\hline 19.47 & 15.00 & 0.27 & 0.28 & 0.29 & 0.18 \\
\hline 19.47 & 15.78 & 0.28 & 0.28 & 0.27 & 0.18 \\
\hline 19.47 & 16.55 & 0.31 & 0.29 & 0.29 & 0.24 \\
\hline 19.47 & 17.33 & 0.32 & 0.32 & 0.31 & 0.24 \\
\hline 19.95 & 2.67 & 0.46 & 0.41 & 0.30 & 0.34 \\
\hline 19.95 & 12.67 & 0.45 & 0.40 & 0.28 & 0.32 \\
\hline 20.42 & 2.67 & 0.24 & 0.28 & 0.29 & 0.20 \\
\hline 20.42 & 12.67 & 0.23 & 0.28 & 0.29 & 0.18 \\
\hline 20.90 & 2.67 & 0.24 & 0.28 & 0.30 & 0.20 \\
\hline 20.90 & 12.67 & 0.21 & 0.27 & 0.29 & 0.18 \\
\hline 21.38 & 2.67 & 0.25 & 0.30 & 0.28 & 0.20 \\
\hline 21.38 & 12.67 & 0.22 & 0.27 & 0.28 & 0.16 \\
\hline 21.85 & 2.67 & 0.26 & 0.31 & 0.27 & 0.22 \\
\hline 21.85 & 12.67 & 0.26 & 0.30 & 0.30 & 0.19 \\
\hline
\end{tabular}


Water Velocity Measurements on an ESBS at John Day Dam

\begin{tabular}{|c|c|c|c|c|c|}
\hline 22.37 & 2.67 & 0.26 & 0.29 & 0.29 & 0.21 \\
\hline 22.37 & 3.45 & 0.25 & 0.29 & 0.31 & 0.23 \\
\hline 22.37 & 4.22 & 0.26 & 0.29 & 0.31 & 0.19 \\
\hline 22.37 & 5.00 & 0.27 & 0.32 & 0.34 & 0.18 \\
\hline 22.37 & 5.78 & 0.26 & 0.29 & 0.30 & 0.20 \\
\hline 22.37 & 6.55 & 0.25 & 0.29 & 0.28 & 0.22 \\
\hline 22.37 & 7.33 & 0.24 & 0.28 & 0.27 & 0.21 \\
\hline 22.37 & 12.67 & 0.23 & 0.27 & 0.28 & 0.18 \\
\hline 22.37 & 13.45 & 0.22 & 0.25 & 0.30 & 0.17 \\
\hline 22.37 & 14.22 & 0.24 & 0.28 & 0.27 & 0.17 \\
\hline 22.37 & 15.00 & 0.23 & 0.28 & 0.27 & 0.17 \\
\hline 22.37 & 15.78 & 0.22 & 0.25 & 0.29 & 0.19 \\
\hline 22.37 & 16.55 & 0.23 & 0.27 & 0.29 & 0.23 \\
\hline 22.37 & 17.33 & 0.23 & 0.27 & 0.28 & 0.22 \\
\hline 22.78 & 7.33 & 0.21 & 0.27 & 0.27 & 0.20 \\
\hline 22.78 & 17.33 & 0.23 & 0.27 & 0.28 & 0.22 \\
\hline 23.19 & 7.33 & 0.23 & 0.28 & 0.28 & 0.19 \\
\hline 23.19 & 17.33 & 0.23 & 0.26 & 0.27 & 0.21 \\
\hline 23.60 & 7.33 & 0.23 & 0.27 & 0.27 & 0.19 \\
\hline 23.60 & 17.33 & 0.22 & 0.25 & 0.28 & 0.20 \\
\hline 24.01 & 7.33 & 0.22 & 0.26 & 0.28 & 0.19 \\
\hline 24.01 & 17.33 & 0.24 & 0.27 & 0.28 & 0.21 \\
\hline 24.42 & 7.33 & 0.29 & 0.33 & 0.28 & 0.22 \\
\hline 24.42 & 17.33 & 0.27 & 0.33 & 0.30 & 0.22 \\
\hline 24.97 & 2.67 & 0.24 & 0.27 & 0.28 & 0.21 \\
\hline 24.97 & 3.45 & 0.23 & 0.29 & 0.28 & 0.19 \\
\hline 24.97 & 4.22 & 0.23 & 0.27 & 0.29 & 0.20 \\
\hline 24.97 & 5.00 & 0.25 & 0.30 & 0.31 & 0.20 \\
\hline 24.97 & 5.78 & 0.26 & 0.33 & 0.28 & 0.21 \\
\hline 24.97 & 6.55 & 0.23 & 0.29 & 0.30 & 0.23 \\
\hline 24.97 & 7.33 & 0.22 & 0.27 & 0.28 & 0.21 \\
\hline 24.97 & 12.67 & 0.23 & 0.26 & 0.28 & 0.18 \\
\hline 24.97 & 13.45 & 0.22 & 0.26 & 0.30 & 0.19 \\
\hline 24.97 & 14.22 & 0.20 & 0.25 & 0.27 & 0.17 \\
\hline 24.97 & 15.00 & 0.23 & 0.27 & 0.27 & 0.20 \\
\hline 24.97 & 15.78 & 0.21 & 0.26 & 0.26 & 0.19 \\
\hline 24.97 & 16.55 & 0.22 & 0.28 & 0.28 & 0.20 \\
\hline 24.97 & 17.33 & 0.22 & 0.27 & 0.29 & 0.19 \\
\hline 25.42 & 2.67 & 0.24 & 0.29 & 0.29 & 0.22 \\
\hline 25.42 & 12.67 & 0.22 & 0.27 & 0.27 & 0.20 \\
\hline 25.87 & 2.67 & 0.19 & 0.26 & 0.28 & 0.18 \\
\hline 25.87 & 12.67 & 0.20 & 0.26 & 0.26 & 0.17 \\
\hline 26.32 & 2.67 & 0.31 & 0.28 & 0.28 & 0.36 \\
\hline 26.32 & 12.67 & 0.28 & 0.32 & 0.30 & 0.41 \\
\hline 26.77 & 2.67 & 0.26 & 0.35 & 0.25 & 0.22 \\
\hline 26.77 & 12.67 & 0.26 & 0.31 & 0.28 & 0.24 \\
\hline 27.22 & 2.67 & 0.19 & 0.25 & 0.27 & 0.15 \\
\hline 27.22 & 12.67 & 0.20 & 0.27 & 0.30 & 0.16 \\
\hline 27.67 & 2.67 & 0.22 & 0.29 & 0.29 & 0.19 \\
\hline 27.67 & 3.45 & 0.26 & 0.35 & 0.36 & 0.19 \\
\hline 27.67 & 4.22 & 0.25 & 0.35 & 0.36 & 0.18 \\
\hline 27.67 & 5.00 & 0.26 & 0.35 & 0.39 & 0.19 \\
\hline 27.67 & 5.78 & 0.26 & 0.35 & 0.35 & 0.20 \\
\hline 27.67 & 6.55 & 0.25 & 0.33 & 0.35 & 0.20 \\
\hline 27.67 & 7.33 & 0.22 & 0.29 & 0.31 & 0.20 \\
\hline 27.67 & 12.67 & 0.23 & 0.28 & 0.29 & 0.20 \\
\hline 27.67 & 13.45 & 0.23 & 0.28 & 0.32 & 0.20 \\
\hline 27.67 & 14.22 & 0.25 & 0.33 & 0.33 & 0.18 \\
\hline 27.67 & 15.00 & 0.25 & 0.34 & 0.31 & 0.19 \\
\hline
\end{tabular}


Water Velocity Measurements on an ESBS at John Day Dam

\begin{tabular}{|c|c|c|c|c|c|}
\hline 27.67 & 15.78 & 0.24 & 0.31 & \begin{tabular}{l|l|}
0.34 \\
\end{tabular} & 0.18 \\
\hline 27.67 & 16.55 & 0.25 & 0.34 & 0.34 & 0.19 \\
\hline 27.67 & 17.33 & 0.23 & 0.32 & 0.30 & 0.19 \\
\hline 28.07 & 7.33 & 0.25 & 0.28 & 0.30 & 0.22 \\
\hline 28.07 & 17.33 & 0.27 & 0.38 & 0.29 & 0.20 \\
\hline 28.47 & 7.33 & 0.27 & 0.29 & 0.27 & 0.21 \\
\hline 28.47 & 17.33 & 0.26 & 0.30 & 0.27 & 0.25 \\
\hline 28.88 & 7.33 & 0.23 & 0.28 & \begin{tabular}{l|l|}
0.30 \\
\end{tabular} & 0.22 \\
\hline 28.88 & 17.33 & 0.26 & 0.32 & 0.29 & 0.24 \\
\hline 29.28 & 7.33 & 0.22 & 0.31 & 0.27 & 0.17 \\
\hline 29.28 & 17.33 & 0.23 & 0.30 & 0.32 & 0.21 \\
\hline 29.68 & 7.33 & 0.24 & 0.35 & 0.24 & 0.14 \\
\hline 29.68 & 17.33 & 0.26 & 0.37 & 0.33 & 0.17 \\
\hline 30.10 & 2.67 & 0.19 & 0.24 & 0.21 & 0.16 \\
\hline 30.10 & 3.45 & 0.19 & 0.24 & 0.21 & 0.15 \\
\hline 30.10 & 4.22 & 0.18 & 0.23 & 0.23 & 0.15 \\
\hline 30.10 & 5.00 & 0.19 & 0.26 & 0.23 & 0.16 \\
\hline 30.10 & 5.78 & 0.19 & 0.26 & \begin{tabular}{l|}
0.21 \\
\end{tabular} & 0.15 \\
\hline 30.10 & 6.55 & 0.18 & 0.23 & 0.22 & 0.14 \\
\hline 30.10 & 7.33 & 0.19 & 0.24 & 0.22 & 0.15 \\
\hline 30.10 & 12.67 & 0.20 & 0.24 & 0.22 & 0.16 \\
\hline 30.10 & 13.45 & 0.19 & 0.23 & 0.21 & 0.15 \\
\hline 30.10 & 14.22 & 0.20 & 0.25 & 0.24 & 0.16 \\
\hline 30.10 & 15.00 & 0.18 & 0.25 & 0.28 & 0.14 \\
\hline 30.10 & 15.78 & 0.20 & 0.23 & 0.24 & 0.14 \\
\hline 30.10 & 16.55 & 0.20 & 0.24 & 0.24 & 0.16 \\
\hline 30.10 & 17.33 & 0.21 & 0.26 & \begin{tabular}{|l|}
0.24 \\
\end{tabular} & 0.17 \\
\hline 30.11 & 2.67 & 0.22 & 0.30 & 0.26 & 0.16 \\
\hline 30.11 & 3.45 & 0.24 & 0.32 & 0.28 & 0.17 \\
\hline 30.11 & 4.22 & 0.19 & 0.27 & 0.25 & 0.15 \\
\hline 30.11 & 5.00 & 0.22 & 0.31 & 0.29 & 0.17 \\
\hline 30.11 & 5.78 & 0.22 & 0.32 & 0.31 & 0.16 \\
\hline 30.11 & 6.55 & 0.23 & 0.32 & 0.28 & 0.16 \\
\hline 30.11 & 7.33 & 0.14 & 0.16 & 0.11 & 0.10 \\
\hline 30.11 & 12.67 & 0.23 & 0.28 & 0.25 & 0.19 \\
\hline 30.11 & 13.45 & 0.23 & 0.30 & 0.27 & 0.18 \\
\hline 30.11 & 14.22 & 0.22 & 0.28 & \begin{tabular}{|l|}
0.28 \\
\end{tabular} & 0.18 \\
\hline 30.11 & 15.00 & 0.23 & 0.30 & 0.28 & 0.18 \\
\hline 30.11 & 15.78 & 0.24 & 0.34 & 0.28 & 0.16 \\
\hline 30.11 & 16.55 & 0.25 & 0.30 & \begin{tabular}{l|}
0.28 \\
\end{tabular} & 0.17 \\
\hline 30.11 & 17.33 & 0.11 & 0.12 & 0.10 & 0.09 \\
\hline 30.20 & 7.33 & 0.14 & 0.18 & 0.12 & 0.07 \\
\hline 30.20 & 17.33 & 0.08 & 0.12 & 0.10 & 0.09 \\
\hline 30.29 & 7.33 & 0.12 & 0.14 & 0.13 & 0.08 \\
\hline 30.29 & 17.33 & 0.11 & 0.07 & 0.12 & 0.10 \\
\hline 30.38 & 7.33 & 0.07 & 0.14 & 0.10 & 0.06 \\
\hline 30.38 & 17.33 & 0.16 & 0.15 & 0.08 & 0.09 \\
\hline 30.46 & 7.33 & 0.10 & 0.08 & \begin{tabular}{l|l|}
0.10 \\
\end{tabular} & 0.10 \\
\hline 30.46 & 17.33 & 0.10 & 0.08 & 0.13 & 0.09 \\
\hline 30.55 & 7.33 & 0.12 & 0.10 & 0.09 & 0.10 \\
\hline 30.55 & 17.33 & 0.13 & 0.16 & 0.06 & 0.08 \\
\hline 30.64 & 2.67 & 0.09 & 0.12 & 0.11 & 0.06 \\
\hline 30.64 & 3.45 & 0.10 & 0.14 & 0.09 & 0.08 \\
\hline 30.64 & 4.22 & 0.09 & 0.10 & \begin{tabular}{l|l}
0.11 \\
\end{tabular} & 0.08 \\
\hline 30.64 & 5.00 & 0.10 & 0.16 & 0.15 & 0.08 \\
\hline 30.64 & 5.78 & 0.12 & 0.17 & 0.15 & 0.09 \\
\hline 30.64 & 6.55 & 0.11 & 0.13 & 0.09 & 0.10 \\
\hline 30.64 & 7.33 & 0.11 & 0.13 & 0.10 & 0.09 \\
\hline 30.64 & 12.67 & 0.10 & 0.12 & 0.12 & 0.09 \\
\hline
\end{tabular}


Water Velocity Measurements on an ESBS at John Day Dam

\begin{tabular}{|c|c|c|c|c|c|}
\hline 30.64 & 13.45 & 0.12 & 0.13 & 0.09 & 0.09 \\
\hline 30.64 & 14.22 & 0.11 & 0.14 & 0.14 & 0.09 \\
\hline 30.64 & 15.00 & 0.10 & 0.11 & 0.18 & 0.09 \\
\hline 30.64 & 15.78 & 0.11 & 0.10 & 0.12 & 0.10 \\
\hline 30.64 & 16.55 & 0.12 & 0.12 & 0.10 & 0.11 \\
\hline 30.64 & 17.33 & 0.14 & 0.14 & 0.10 & 0.09 \\
\hline 30.69 & 2.67 & 0.11 & 0.13 & 0.05 & 0.09 \\
\hline 30.69 & 12.67 & 0.12 & 0.13 & 0.11 & 0.08 \\
\hline 30.78 & 2.67 & 0.11 & 0.10 & 0.11 & 0.08 \\
\hline 30.78 & 12.67 & 0.10 & 0.12 & 0.11 & 0.08 \\
\hline 30.86 & 2.67 & 0.14 & 0.08 & 0.11 & 0.17 \\
\hline 30.86 & 12.67 & 0.20 & 0.15 & 0.13 & 0.25 \\
\hline 30.94 & 2.67 & 0.05 & 0.08 & 0.09 & 0.05 \\
\hline 30.94 & 12.67 & 0.10 & 0.10 & 0.06 & 0.08 \\
\hline 31.03 & 2.67 & 0.06 & 0.11 & 0.07 & 0.03 \\
\hline 31.03 & 12.67 & 0.06 & 0.19 & 0.11 & 0.11 \\
\hline 31.11 & 2.67 & 0.09 & 0.11 & 0.12 & 0.08 \\
\hline 31.11 & 3.45 & 0.09 & 0.13 & 0.13 & 0.07 \\
\hline 31.11 & 4.22 & 0.09 & 0.11 & 0.12 & 0.09 \\
\hline 31.11 & 5.00 & 0.10 & 0.16 & 0.17 & 0.09 \\
\hline 31.11 & 5.78 & 0.10 & 0.16 & 0.13 & 0.08 \\
\hline 31.11 & 6.55 & 0.12 & 0.16 & 0.12 & 0.09 \\
\hline 31.11 & 7.33 & 0.11 & 0.17 & 0.12 & 0.08 \\
\hline 31.11 & 12.67 & 0.10 & 0.14 & 0.10 & 0.09 \\
\hline 31.11 & 13.45 & 0.11 & 0.14 & 0.12 & 0.10 \\
\hline 31.11 & 14.22 & 0.11 & 0.20 & 0.14 & 0.08 \\
\hline 31.11 & 15.00 & 0.11 & 0.17 & 0.12 & 0.08 \\
\hline 31.11 & 15.78 & 0.09 & 0.09 & 0.11 & 0.08 \\
\hline 31.11 & 16.55 & 0.09 & 0.10 & 0.11 & 0.09 \\
\hline 31.11 & 17.33 & 0.09 & 0.10 & 0.11 & 0.08 \\
\hline 31.19 & 7.33 & 0.14 & 0.16 & 0.23 & 0.13 \\
\hline 31.19 & 17.33 & 0.06 & 0.08 & 0.08 & 0.04 \\
\hline 31.28 & 7.33 & 0.07 & 0.10 & 0.07 & 0.05 \\
\hline 31.28 & 17.33 & 0.06 & 0.11 & 0.06 & 0.09 \\
\hline 31.36 & 7.33 & 0.15 & 0.20 & 0.08 & 0.14 \\
\hline 31.36 & 17.33 & 0.18 & 0.12 & 0.09 & 0.15 \\
\hline 31.44 & 7.33 & 0.10 & 0.17 & 0.09 & 0.14 \\
\hline 31.44 & 17.33 & 0.12 & 0.05 & 0.10 & 0.12 \\
\hline 31.53 & 7.33 & 0.12 & 0.21 & 0.15 & 0.15 \\
\hline 31.53 & 17.33 & 0.15 & 0.14 & 0.13 & 0.17 \\
\hline 31.61 & 2.67 & 0.10 & 0.11 & 0.12 & 0.08 \\
\hline 31.61 & 3.45 & 0.09 & 0.12 & 0.11 & 0.07 \\
\hline 31.61 & 4.22 & 0.07 & 0.10 & 0.10 & 0.07 \\
\hline 31.61 & 5.00 & 0.10 & 0.15 & 0.20 & 0.09 \\
\hline 31.61 & 5.78 & 0.09 & 0.16 & 0.14 & 0.09 \\
\hline 31.61 & 6.55 & 0.10 & 0.15 & 0.13 & 0.10 \\
\hline 31.61 & 7.33 & 0.09 & 0.14 & 0.12 & 0.08 \\
\hline 31.61 & 12.67 & 0.10 & 0.14 & 0.12 & 0.09 \\
\hline 31.61 & 13.45 & 0.09 & 0.17 & 0.16 & 0.09 \\
\hline 31.61 & 14.22 & 0.11 & 0.14 & 0.14 & 0.09 \\
\hline 31.61 & 15.00 & 0.12 & 0.17 & 0.13 & 0.08 \\
\hline 31.61 & 15.78 & 0.11 & 0.11 & 0.11 & 0.09 \\
\hline 31.61 & 16.55 & 0.11 & 0.13 & 0.11 & 0.08 \\
\hline 31.61 & 17.33 & 0.09 & 0.12 & 0.10 & 0.08 \\
\hline 31.67 & 2.67 & 0.11 & 0.12 & 0.12 & 0.07 \\
\hline 31.67 & 12.67 & 0.13 & 0.12 & 0.15 & 0.10 \\
\hline 31.72 & 2.67 & 0.09 & 0.10 & 0.09 & 0.09 \\
\hline 31.72 & 12.67 & 0.12 & 0.15 & 0.13 & 0.10 \\
\hline 31.78 & 2.67 & 0.16 & 0.20 & 0.10 & 0.08 \\
\hline
\end{tabular}


Water Velocity Measurements on an ESBS at John Day Dam

\begin{tabular}{|c|c|c|c|c|c|}
\hline 31.78 & 12.67 & 0.14 & 0.16 & \begin{tabular}{l|l}
0.11 \\
\end{tabular} & 0.11 \\
\hline 31.84 & 2.67 & 0.12 & 0.15 & 0.07 & 0.07 \\
\hline 31.84 & 12.67 & 0.13 & 0.13 & 0.14 & 0.15 \\
\hline 31.89 & 2.67 & 0.11 & 0.15 & 0.09 & 0.09 \\
\hline 31.89 & 12.67 & 0.07 & 0.14 & \begin{tabular}{|l|}
0.13 \\
\end{tabular} & 0.07 \\
\hline 31.95 & 2.67 & 0.10 & 0.11 & 0.11 & 0.08 \\
\hline 31.95 & 3.45 & 0.09 & 0.15 & 0.17 & 0.09 \\
\hline 31.95 & 4.22 & 0.09 & 0.11 & \begin{tabular}{l|}
0.11 \\
\end{tabular} & 0.08 \\
\hline 31.95 & 5.00 & 0.10 & 0.14 & 0.10 & 0.07 \\
\hline 31.95 & 5.78 & 0.11 & 0.18 & 0.25 & 0.08 \\
\hline 31.95 & 6.55 & 0.13 & 0.14 & 0.14 & 0.10 \\
\hline 31.95 & 7.33 & 0.09 & 0.15 & 0.12 & 0.09 \\
\hline 31.95 & 12.67 & 0.10 & 0.13 & 0.13 & 0.09 \\
\hline 31.95 & 13.45 & 0.11 & 0.14 & \begin{tabular}{l|l|}
0.14 \\
\end{tabular} & 0.10 \\
\hline 31.95 & 14.22 & 0.11 & 0.17 & 0.13 & 0.08 \\
\hline 31.95 & 15.00 & 0.09 & 0.13 & 0.17 & 0.09 \\
\hline 31.95 & 15.78 & 0.18 & 0.24 & 0.15 & 0.10 \\
\hline 31.95 & 16.55 & 0.10 & 0.12 & 0.12 & 0.10 \\
\hline 31.95 & 17.33 & 0.11 & 0.14 & 0.11 & 0.09 \\
\hline 32.02 & 7.33 & 0.13 & 0.18 & 0.10 & 0.11 \\
\hline 32.02 & 17.33 & 0.12 & 0.15 & 0.10 & 0.05 \\
\hline 32.10 & 7.33 & 0.14 & 0.15 & 0.15 & 0.10 \\
\hline 32.10 & 17.33 & 0.12 & 0.12 & 0.06 & 0.08 \\
\hline 32.18 & 7.33 & 0.08 & 0.15 & 0.15 & 0.05 \\
\hline 32.18 & 17.33 & 0.11 & 0.14 & 0.15 & 0.07 \\
\hline 32.25 & 7.33 & 0.08 & 0.14 & 0.11 & 0.10 \\
\hline 32.25 & 17.33 & 0.11 & 0.09 & 0.12 & 0.09 \\
\hline 32.33 & 7.33 & 0.09 & 0.10 & \begin{tabular}{l|l|}
0.13 \\
\end{tabular} & 0.10 \\
\hline 32.33 & 17.33 & 0.08 & 0.10 & 0.11 & 0.06 \\
\hline 32.41 & 2.67 & 0.10 & 0.13 & 0.10 & 0.08 \\
\hline 32.41 & 3.45 & 0.08 & 0.11 & 0.10 & 0.07 \\
\hline 32.41 & 4.22 & 0.11 & 0.14 & 0.13 & 0.09 \\
\hline 32.41 & 5.00 & 0.09 & 0.12 & 0.15 & 0.08 \\
\hline 32.41 & 5.78 & 0.17 & 0.26 & 0.18 & 0.08 \\
\hline 32.41 & 6.55 & 0.11 & 0.19 & 0.15 & 0.08 \\
\hline 32.41 & 7.33 & 0.10 & 0.14 & 0.12 & 0.09 \\
\hline 32.41 & 12.67 & 0.09 & 0.13 & \begin{tabular}{|l|}
0.11 \\
\end{tabular} & 0.09 \\
\hline 32.41 & 13.45 & 0.10 & 0.13 & 0.13 & 0.09 \\
\hline 32.41 & 14.22 & 0.10 & 0.13 & 0.14 & 0.09 \\
\hline 32.41 & 15.00 & 0.14 & 0.25 & \begin{tabular}{l|}
0.16 \\
\end{tabular} & 0.09 \\
\hline 32.41 & 15.78 & 0.13 & 0.18 & 0.26 & 0.08 \\
\hline 32.41 & 16.55 & 0.10 & 0.11 & 0.15 & 0.09 \\
\hline 32.41 & 17.33 & 0.10 & 0.13 & 0.10 & 0.10 \\
\hline 32.48 & 2.67 & 0.03 & 0.07 & 0.05 & 0.07 \\
\hline 32.48 & 12.67 & 0.06 & 0.20 & 0.13 & 0.12 \\
\hline 32.56 & 2.67 & 0.08 & 0.05 & 0.05 & 0.07 \\
\hline 32.56 & 12.67 & 0.08 & 0.11 & 0.03 & 0.02 \\
\hline 32.64 & 2.67 & 0.10 & 0.10 & 0.03 & 0.08 \\
\hline 32.64 & 12.67 & 0.06 & 0.11 & 0.06 & 0.16 \\
\hline 32.71 & 2.67 & 0.00 & 0.13 & 0.06 & 0.09 \\
\hline 32.71 & 12.67 & 0.03 & 0.01 & 0.04 & 0.03 \\
\hline 32.79 & 2.67 & 0.01 & 0.08 & 0.08 & 0.06 \\
\hline 32.79 & 12.67 & 0.08 & 0.01 & 0.02 & 0.10 \\
\hline 32.87 & 2.67 & 0.09 & 0.12 & 0.11 & 0.08 \\
\hline 32.87 & 3.45 & 0.09 & 0.11 & 0.16 & 0.09 \\
\hline 32.87 & 4.22 & 0.08 & 0.12 & 0.11 & 0.07 \\
\hline 32.87 & 5.00 & 0.11 & 0.19 & 0.15 & 0.07 \\
\hline 32.87 & 5.78 & 0.10 & 0.19 & 0.11 & 0.07 \\
\hline 32.87 & 6.55 & 0.09 & 0.14 & 0.13 & 0.08 \\
\hline
\end{tabular}


Water Velocity Measurements on an ESBS at John Day Dam

\begin{tabular}{|c|c|c|c|c|c|}
\hline 32.87 & 7.33 & 0.10 & 0.13 & 0.12 & 0.09 \\
\hline 32.87 & 12.67 & 0.10 & 0.15 & 0.13 & 0.08 \\
\hline 32.87 & 13.45 & 0.10 & 0.12 & 0.15 & 0.10 \\
\hline 32.87 & 14.22 & 0.10 & 0.19 & 0.15 & 0.08 \\
\hline 32.87 & 15.00 & 0.11 & 0.19 & 0.19 & 0.08 \\
\hline 32.87 & 15.78 & 0.08 & 0.15 & 0.14 & 0.06 \\
\hline 32.87 & 16.55 & 0.09 & 0.12 & 0.13 & 0.08 \\
\hline 32.87 & 17.33 & 0.08 & 0.11 & 0.10 & 0.07 \\
\hline 32.95 & 2.67 & 0.08 & 0.13 & 0.11 & 0.07 \\
\hline 32.95 & 3.45 & 0.09 & 0.12 & 0.11 & 0.09 \\
\hline 32.95 & 4.22 & 0.08 & 0.13 & 0.15 & 0.07 \\
\hline 32.95 & 5.00 & 0.11 & 0.17 & 0.23 & 0.08 \\
\hline 32.95 & 5.78 & 0.09 & 0.13 & 0.13 & 0.07 \\
\hline 32.95 & 6.55 & 0.11 & 0.14 & 0.14 & 0.09 \\
\hline 32.95 & 7.33 & 0.11 & 0.16 & 0.13 & 0.09 \\
\hline 32.95 & 12.67 & 0.10 & 0.15 & 0.11 & 0.09 \\
\hline 32.95 & 13.45 & 0.10 & 0.16 & 0.15 & 0.10 \\
\hline 32.95 & 14.22 & 0.09 & 0.19 & 0.16 & 0.06 \\
\hline 32.95 & 15.00 & 0.12 & 0.23 & 0.12 & 0.08 \\
\hline 32.95 & 15.78 & 0.10 & 0.13 & 0.13 & 0.08 \\
\hline 32.95 & 16.55 & 0.12 & 0.14 & 0.13 & 0.09 \\
\hline 32.95 & 17.33 & 0.10 & 0.13 & 0.11 & 0.08 \\
\hline 33.31 & 7.33 & 0.11 & 0.14 & 0.13 & 0.09 \\
\hline 33.31 & 17.33 & 0.11 & 0.14 & 0.13 & 0.07 \\
\hline 33.66 & 7.33 & 0.51 & 0.28 & 0.19 & 0.63 \\
\hline 33.66 & 17.33 & 0.50 & 0.32 & 0.16 & 0.63 \\
\hline 34.02 & 7.33 & 0.09 & 0.18 & 0.13 & 0.09 \\
\hline 34.02 & 17.33 & 0.08 & 0.14 & 0.12 & 0.08 \\
\hline 34.37 & 7.33 & 0.07 & 0.14 & 0.14 & 0.06 \\
\hline 34.37 & 17.33 & 0.08 & 0.15 & 0.17 & 0.10 \\
\hline 34.73 & 7.33 & 0.08 & 0.15 & 0.12 & 0.07 \\
\hline 34.73 & 17.33 & 0.08 & 0.17 & 0.12 & 0.11 \\
\hline 35.08 & 2.67 & 0.16 & 0.33 & 0.34 & 0.16 \\
\hline 35.08 & 3.45 & 0.17 & 0.37 & 0.38 & 0.17 \\
\hline 35.08 & 4.22 & 0.17 & 0.36 & 0.36 & 0.16 \\
\hline 35.08 & 5.00 & 0.19 & 0.40 & 0.39 & 0.17 \\
\hline 35.08 & 5.78 & 0.19 & 0.41 & 0.36 & 0.18 \\
\hline 35.08 & 6.55 & 0.19 & 0.40 & 0.39 & 0.17 \\
\hline 35.08 & 7.33 & 0.19 & 0.41 & 0.41 & 0.19 \\
\hline 35.08 & 12.67 & 0.17 & 0.34 & 0.33 & 0.18 \\
\hline 35.08 & 13.45 & 0.15 & 0.32 & 0.33 & 0.17 \\
\hline 35.08 & 14.22 & 0.16 & 0.33 & 0.35 & 0.17 \\
\hline 35.08 & 15.00 & 0.15 & 0.30 & 0.34 & 0.15 \\
\hline 35.08 & 15.78 & 0.16 & 0.34 & 0.34 & 0.16 \\
\hline 35.08 & 16.55 & 0.18 & 0.35 & 0.35 & 0.17 \\
\hline 35.08 & 17.33 & 0.19 & 0.36 & 0.36 & 0.19 \\
\hline 35.21 & 2.67 & 0.16 & 0.33 & 0.31 & 0.16 \\
\hline 35.21 & 12.67 & 0.16 & 0.30 & 0.32 & 0.16 \\
\hline 35.35 & 2.67 & 0.15 & 0.35 & 0.30 & 0.15 \\
\hline 35.35 & 12.67 & 0.16 & 0.31 & 0.31 & 0.16 \\
\hline 35.49 & 2.67 & 0.16 & 0.36 & 0.31 & 0.15 \\
\hline 35.49 & 12.67 & 0.16 & 0.32 & 0.31 & 0.16 \\
\hline 35.62 & 2.67 & 0.15 & 0.33 & 0.31 & 0.15 \\
\hline 35.62 & 12.67 & 0.16 & 0.30 & 0.32 & 0.16 \\
\hline 35.76 & 2.67 & 0.14 & 0.33 & 0.31 & 0.14 \\
\hline 35.76 & 12.67 & 0.15 & 0.30 & 0.32 & 0.15 \\
\hline 35.89 & 2.67 & 0.14 & 0.35 & 0.32 & 0.14 \\
\hline 35.89 & 3.45 & 0.14 & 0.35 & 0.37 & 0.14 \\
\hline 35.89 & 4.22 & 0.14 & 0.33 & 0.36 & 0.14 \\
\hline
\end{tabular}


Water Velocity Measurements on an ESBS at John Day Dam

\begin{tabular}{|r|r|r|r|r|r|}
\hline 35.89 & 5.00 & 0.14 & 0.34 & 0.33 & 0.14 \\
\hline 35.89 & 5.78 & 0.16 & 0.38 & 0.34 & 0.16 \\
\hline 35.89 & 6.55 & 0.16 & 0.37 & 0.35 & 0.16 \\
\hline 35.89 & 7.33 & 0.16 & 0.39 & 0.32 & 0.16 \\
\hline 35.89 & 12.67 & 0.16 & 0.36 & 0.34 & 0.16 \\
\hline 35.89 & 13.45 & 0.15 & 0.36 & 0.37 & 0.15 \\
\hline 35.89 & 14.22 & 0.15 & 0.35 & 0.36 & 0.16 \\
\hline 35.89 & 15.00 & 0.16 & 0.35 & 0.33 & 0.16 \\
\hline 35.89 & 15.78 & 0.15 & 0.36 & 0.33 & 0.16 \\
\hline 35.89 & 16.55 & 0.17 & 0.37 & 0.37 & 0.17 \\
\hline 35.89 & 17.33 & 0.16 & 0.39 & 0.35 & 0.17 \\
\hline
\end{tabular}




\section{Appendix D}

\section{$\mathrm{X}, \mathrm{Y}$, and Z Vector Velocities at $138 \mathrm{MW}$ Turbine Load}




\section{Appendix D}

\section{$\mathrm{X}, \mathrm{Y}$, and Z Vector Velocities at $138 \mathrm{MW}$ Turbine Load}

The table in this appendix lists $\mathrm{X}, \mathrm{Y}$, and $\mathrm{Z}$ vector velocities ( $\mathrm{ft} / \mathrm{sec}$ ) for flow data collected at locations along the bar screen surface of the ESBS at $138 \mathrm{MW}$ turbine load. Mean, minimum, and maximum vector velocities and standard error are provided for each sample location.

\begin{tabular}{|c|c|c|c|c|c|c|c|c|c|c|c|c|c|}
\hline \multicolumn{2}{|c|}{ Location (ft.) } & \multicolumn{4}{|c|}{ X Vector (ft/sec) } & \multicolumn{4}{|c|}{ Y Vector (ft/sec) } & \multicolumn{4}{|c|}{ Z Vector (ft/sec) } \\
\hline Vertical & Horizontal & Mean & Min & Max & Stderr & Mean & Min & Max & Stderr & Mean & Min & Max & Stderr \\
\hline 0.03 & 2.67 & 5.34 & 3.08 & 7.40 & 0.02000 & 0.31 & -2.11 & 2.75 & 0.02000 & -3.94 & -5.31 & -2.53 & 0.01000 \\
\hline 0.03 & 3.45 & 5.13 & 4.41 & 5.97 & 0.03083 & 0.23 & -0.30 & 0.81 & 0.02254 & -4.07 & -4.69 & -3.44 & 0.02578 \\
\hline 0.03 & 4.22 & 4.47 & 3.88 & 5.09 & 0.02481 & 0.76 & -0.06 & 1.29 & 0.02577 & -4.09 & -4.63 & -3.41 & 0.02951 \\
\hline 0.03 & 5.00 & 4.38 & 3.88 & 5.32 & 0.02804 & 0.53 & -0.32 & 1.44 & 0.03144 & -3.33 & -4.07 & -2.65 & 0.02767 \\
\hline 0.03 & 5.78 & 5.10 & 4.25 & 5.99 & 0.03182 & -0.06 & -0.66 & 0.54 & 0.01903 & -3.34 & -3.92 & -2.80 & 0.02182 \\
\hline 0.03 & 6.55 & 5.36 & 4.83 & 6.17 & 0.02396 & -0.10 & -0.53 & 0.48 & 0.01842 & -3.62 & -4.14 & -2.93 & 0.02020 \\
\hline 0.03 & 7.33 & 5.48 & 5.04 & 6.05 & 0.03000 & -0.06 & -0.54 & 0.42 & 0.02000 & -3.71 & -4.31 & -3.08 & 3000 \\
\hline 0.03 & 12.67 & 5.56 & 4.05 & 6.98 & 0.01000 & 0.95 & -0.70 & 2.39 & 0.01000 & -3.79 & -4.96 & -2.52 & 0.01000 \\
\hline 0.03 & 13.45 & 5.56 & 4.89 & 6.63 & 0.02514 & 0.97 & 0.15 & 1.77 & 0.02179 & -3.52 & -4.07 & -2.72 & 0.02731 \\
\hline 0.03 & 14.22 & 4.97 & 4.17 & 5.95 & 0.03845 & 0.65 & -0.40 & 1.36 & 3039 & -3.17 & -3.83 & -2.54 & 444 \\
\hline 0.03 & 15.00 & 4.47 & 3.94 & 5.03 & 0.02172 & -0.23 & -0.71 & 0.58 & 0.02467 & -3.75 & -4.55 & -2.98 & 3502 \\
\hline 0.03 & 15.78 & 4.84 & 3.88 & 5.72 & 0.03490 & 0.37 & -0.62 & 1.31 & 0.04168 & -4.34 & -4.92 & -3.67 & 410 \\
\hline 0.03 & 16.55 & 5.51 & 4.85 & 6.53 & 362 & 0.85 & -0.02 & 1.76 & 848 & -3.84 & -4.49 & -3.12 & 888 \\
\hline 0.03 & 17.33 & 5.61 & 4.52 & 6.49 & 0.04000 & 0.81 & -0.80 & 1.52 & 0.04000 & -3.91 & -4.80 & -3.30 & 4000 \\
\hline 0.35 & 2.67 & 5.38 & 3.72 & 6.99 & 0.03146 & 0.32 & -0.96 & 1.98 & 3091 & -4.07 & -5.23 & -2.79 & 169 \\
\hline 0.35 & 12.67 & 5.65 & 4.34 & 6.97 & 0.0 & 1.07 & -0.09 & 2.00 & 332 & -3.82 & .17 & -2.74 & 321 \\
\hline 0.66 & 2.67 & 5.38 & 3.24 & 6.93 & 0.02993 & 0.34 & -0.98 & 1.99 & 0.02778 & -4.02 & -5.22 & -2.97 & 194 \\
\hline 0 & 12.67 & 5.57 & 4.11 & 6.96 & 0.0 & 0.95 & -0.13 & & 323 & -3.89 & 97 & -2.76 & 235 \\
\hline 0.98 & 2.67 & 5.54 & 3.73 & 6.94 & 897 & 0.23 & -0.99 & 1.98 & 893 & -3.99 & 5.24 & -2.64 & 440 \\
\hline 0.98 & 12.67 & 5.61 & 4.04 & 6.98 & 665 & 0.95 & -0.11 & 1.98 & 2288 & -3.88 & -4.87 & -2.35 & 2133 \\
\hline 1.29 & 2.67 & 5.02 & 2.71 & 6.96 & 0.0 & 0.37 & -0.98 & 1.91 & 0.02832 & -3.86 & -5.44 & -2.54 & 559 \\
\hline 1.29 & 12.67 & 5.23 & 3.24 & 6.98 & 357 & 0.95 & -0.06 & 1.99 & 0.02363 & -3.70 & -4.84 & -2.37 & 2504 \\
\hline 1.61 & 2.67 & 3.41 & 2.01 & 5.07 & 636 & 0.14 & -0.99 & 1.89 & 523 & -1.99 & -4.23 & -0.72 & 077 \\
\hline 1.61 & 12.67 & 3.54 & 2.28 & 5.35 & 0.0 & 0.62 & -0.15 & & 59 & -1.99 & 87 & -0 & 472 \\
\hline 1.96 & 2.67 & 3.33 & 2.04 & 4.46 & 482 & 0.17 & -0.74 & 1.59 & 572 & -1.57 & -2.30 & -0.63 & 1072 \\
\hline 1.96 & 3.45 & 3.26 & 1.66 & 4.97 & & 0.15 & -0.99 & & 315 & -1.61 & -2.59 & -0.59 & \\
\hline 1.96 & 4.22 & 3.22 & 1.47 & 4.86 & & 0.32 & -1.00 & & 426 & -1.71 & -2.58 & -0.90 & \\
\hline 1.96 & 5.00 & 3.12 & 1.30 & 4.62 & 258 & 0.55 & -0.96 & 1.97 & 352 & -1.41 & -2.36 & -0.43 & 933 \\
\hline 1.96 & 5.78 & 3.23 & 1.82 & 4.75 & 0.01246 & 0.25 & -1.00 & 1.77 & 0.01271 & -1.18 & -2.17 & -0.34 & 919 \\
\hline 1.9 & 6.55 & 3.37 & 1.86 & 4.65 & 37 & 0.19 & -0.90 & 1.83 & 233 & -1.20 & -1.98 & -0.35 & 855 \\
\hline 1.96 & 7.33 & 3.38 & 2.04 & 4.36 & 348 & 0.17 & -0.87 & 1.60 & 1404 & -1.21 & -1.90 & -0.51 & 0984 \\
\hline 1.96 & 12.67 & 3.33 & 2.22 & 4.43 & 0.01287 & 0.58 & -0.72 & 1.59 & 0.01378 & -1.56 & -2.41 & -0.62 & 1089 \\
\hline 1.96 & 13.45 & 3.37 & 1.78 & 4.61 & 114 & 0.54 & -0.83 & 1.99 & 0.01215 & -1.50 & -2.55 & -0.45 & 956 \\
\hline 1.96 & 14.22 & 3.47 & 2.23 & 4.94 & 0.01172 & 0.60 & -0.94 & 1.87 & 0.01214 & -1.32 & -2.26 & -0.32 & 0.00988 \\
\hline 1.96 & 15.00 & 3.14 & 1.91 & 4.80 & 0.01135 & 0.32 & -0.89 & 1.82 & 0.01325 & -1.32 & -2.21 & -0.40 & 0.00913 \\
\hline 1.96 & 15.78 & 3.07 & 1.88 & 4.41 & 0.01102 & 0.38 & -0.89 & 1.90 & 0.01367 & -1.54 & -2.29 & -0.42 & 0.00768 \\
\hline 1.96 & 16.55 & 2.98 & 1.68 & 4.42 & 0.01305 & 0.77 & -0.86 & 1.99 & 0.01428 & -1.40 & -2.40 & -0.29 & 0.01079 \\
\hline 1.96 & 17.33 & 3.10 & 2.04 & 4.21 & & 0.84 & -0.81 & 1.86 & 0.01663 & -1.28 & -2.00 & -0.51 & 0.01121 \\
\hline 6.41 & 2.67 & 2.69 & 1.51 & 3.85 & 0.00954 & 0.23 & -0.50 & 1.42 & 0.00956 & -0.09 & -0.60 & 0.65 & 0.00564 \\
\hline 6.41 & 3.45 & 2.65 & 1.06 & 3.80 & 0.01327 & 0.14 & -0.94 & 1.84 & 0.01343 & -0.17 & -0.81 & 0.49 & 0.00795 \\
\hline 6.41 & 4.22 & 2.69 & 1.20 & 3.84 & 0.01388 & 0.30 & -0.79 & 1.92 & 0.01423 & -0.30 & -0.96 & 0.47 & 0.00799 \\
\hline 6.41 & 5.00 & 2.41 & 1.05 & 3.93 & 0.01404 & 0.50 & -0.61 & 1.82 & 0.01331 & -0.30 & -0.88 & 0.60 & 0.00820 \\
\hline 6.41 & 5.78 & 2.50 & 1.05 & 3.85 & 0.01472 & 0.28 & -0.94 & 1.43 & 0.01349 & -0.10 & -0.94 & 0.83 & 0.01120 \\
\hline 6.41 & 6.55 & 2.83 & 1.05 & 3.95 & & 0.44 & -0.80 & 1.65 & 0.01241 & 0.17 & -0.59 & 0.95 & 0.01014 \\
\hline 6.41 & 7.33 & 2.90 & 1.58 & 3.97 & 0.00957 & 0.42 & -0.50 & 1.57 & 0.00962 & 0.31 & -0.70 & 1.34 & 0.00781 \\
\hline
\end{tabular}


Water Velocity Measurements on an ESBS at John Day Dam

\begin{tabular}{|l|l|l|l|l|l|l|l|l|l|l|l|l|l|}
\hline 6.41 & 12.67 & 2.88 & 1.78 & 3.97 & 0.00858 & 0.62 & -0.41 & 1.50 & 0.00810 & 0.32 & -0.44 & 0.99 & 0.00613 \\
\hline 6.41 & 13.45 & 2.91 & 1.78 & 3.88 & 0.01268 & 0.66 & -0.46 & 1.94 & 0.01176 & 0.45 & -0.41 & 1.22 & 0.00979 \\
\hline 6.41 & 14.22 & 2.89 & 1.87 & 3.92 & 0.01320 & 0.79 & -0.39 & 1.79 & 0.01204 & 0.56 & -0.40 & 1.21 & 0.00901 \\
\hline 6.41 & 15.00 & 2.33 & 1.14 & 3.62 & 0.01322 & 0.48 & -0.60 & 1.42 & 0.01180 & 0.42 & -0.22 & 1.21 & 0.00943 \\
\hline 6.41 & 15.78 & 2.46 & 1.16 & 3.81 & 0.01224 & 0.48 & -0.85 & 1.58 & 0.01185 & 0.38 & -0.48 & 1.17 & 0.00883 \\
\hline 6.41 & 16.55 & 2.49 & 1.35 & 3.94 & 0.01332 & 0.72 & -0.66 & 1.88 & 0.01397 & 0.60 & -0.23 & 1.54 & 0.01006 \\
\hline
\end{tabular}




\section{Appendix E}

\section{Spherical Coordinates for Flow Data at 138 MW Turbine Load}




\section{Appendix E}

\section{Spherical Coordinates for Flow Data at 138 MW Turbine Load}

The table in this appendix lists spherical coordinates for flow data collected at locations along the bar screen surface of the ESBS at $138 \mathrm{MW}$ turbine load, where Rho is the magnitude flow velocity ( $\mathrm{ft} / \mathrm{sec}$ ) and phi and theta are angles of the flow.

\begin{tabular}{|c|c|c|c|c|c|c|c|c|c|c|c|c|c|}
\hline \multicolumn{2}{|c|}{ Location (ft.) } & \multicolumn{4}{|c|}{ Rho (magnitude velocity) } & \multicolumn{4}{|l|}{ Phi } & \multicolumn{4}{|l|}{ Theta } \\
\hline Vertical & Horizontal & Mean & Min & Max & Stderr & Mean & Min & Max & Stderr & Mean & Min & Max & Stderr \\
\hline 0.03 & 2.67 & 6.71 & 4.77 & 8.39 & 0.01056 & 126.35 & 109.34 & 145.34 & 0.12098 & 3.42 & -30.04 & 39.67 & 0.18516 \\
\hline 0.03 & 3.45 & 6.57 & 6.26 & 6.97 & 0.01336 & 128.37 & 121.08 & 135.68 & 0.32612 & 2.59 & -3.35 & 10.07 & 0.26309 \\
\hline 0.03 & 4.22 & 6.12 & 5.47 & 6.59 & 0.02503 & 131.95 & 124.51 & 138.51 & 0.27067 & 9.61 & -0.63 & 15.82 & 0.33138 \\
\hline 0.03 & 5.00 & 5.55 & 5.16 & 6.05 & 0.01697 & 127.01 & 118.43 & 134.90 & 0.36213 & 6.91 & -3.92 & 18.57 & 0.40743 \\
\hline 0.03 & 5.78 & 6.11 & 5.48 & 6.75 & 0.02404 & 123.25 & 116.44 & 130.88 & 0.28331 & -0.62 & -7.03 & 5.84 & 0.21362 \\
\hline 0.03 & 6.55 & 6.48 & 6.05 & 6.83 & 0.01323 & 124.06 & 115.43 & 129.96 & 0.24938 & -1.08 & -6.03 & 5.63 & 0.20016 \\
\hline 0.03 & 7.33 & 6.63 & 6.33 & 7.01 & 0.01466 & 124.10 & 117.34 & 130.17 & 0.29600 & -0.66 & -5.57 & 4.61 & 0.22845 \\
\hline 0.03 & 12.67 & 6.85 & 5.54 & 8.13 & 0.00726 & 123.91 & 109.94 & 137.71 & 0.09656 & 9.62 & -9.32 & 28.94 & 0.10155 \\
\hline 0.03 & 13.45 & 6.67 & 6.28 & 7.49 & 0.01660 & 121.97 & 113.46 & 129.21 & 0.29038 & 9.87 & 1.58 & 15.59 & 0.21103 \\
\hline 0.03 & 14.22 & 5.95 & 5.32 & 6.59 & 0.02973 & 122.41 & 113.01 & 131.63 & 0.34581 & 7.37 & -4.66 & 14.96 & 0.32994 \\
\hline 0.03 & 15.00 & 5.85 & 5.29 & 6.52 & 0.02629 & 129.87 & 121.93 & 138.30 & 0.30905 & -2.97 & -9.46 & 7.87 & 0.32169 \\
\hline 0.03 & 15.78 & 6.54 & 6.12 & 7.13 & 0.01749 & 131.73 & 123.28 & 141.63 & 0.35375 & 4.15 & -7.72 & 14.12 & 0.47316 \\
\hline 0.03 & 16.55 & 6.79 & 6.34 & 7.36 & 0.01897 & 124.64 & 115.97 & 131.55 & 150 & 8.66 & -0.26 & 16.49 & 0.26701 \\
\hline 0.03 & 17.33 & 6.91 & 6.33 & 7.51 & 0.02356 & 124.63 & 116.36 & 135.83 & 0.42590 & 8.10 & -10.01 & 13.77 & 0.37454 \\
\hline 0.35 & 2.67 & 6.80 & 5.62 & 7.90 & 0.02396 & 127.05 & 113.75 & 138.50 & 0.24231 & 3.45 & -11.14 & 25.81 & 0.34527 \\
\hline 0.35 & 12.67 & 6.94 & 5.80 & 7.88 & 0.01821 & 123.63 & 111.96 & 136.14 & 0.24832 & 10.67 & -0.86 & 20.53 & 0.22766 \\
\hline 0.66 & 2.67 & 6.77 & 5.47 & 8.00 & 0.02171 & 126.72 & 114.45 & 143.58 & 0.25315 & 3.61 & -13.19 & 26.64 & 0.30622 \\
\hline 0.66 & 12.67 & 6.90 & 5.66 & 8.24 & 0.02075 & 124.56 & 111.94 & 136.39 & 550 & 9.69 & -1.66 & 21.40 & 507 \\
\hline 0.98 & 2.67 & 6.88 & 5.52 & 8.27 & 0.02167 & 125.67 & 112.40 & 138.14 & 488 & 2.41 & -10.78 & 27.82 & 891 \\
\hline 0.98 & 12.67 & 6.92 & 5.85 & 8.08 & 0.01899 & 124.28 & 110.49 & 136.37 & 0.23929 & 9.56 & -1.32 & 21.78 & 0.22561 \\
\hline 1.29 & 2.67 & 6.40 & 4.20 & 7.86 & 0.03262 & 127.60 & 112.87 & 146.65 & 0.30482 & 4.36 & -10.39 & 28.61 & 729 \\
\hline 1.29 & 12.67 & 6.52 & 4.76 & 7.89 & 0.02909 & 124.90 & 108.49 & 142.39 & 0.27072 & 10.32 & -0.74 & 23.80 & 0.25167 \\
\hline 1.61 & 2.67 & 4.04 & 2.75 & 6.24 & 0.03322 & 119.26 & 101.29 & 148.16 & 0.48742 & 2.36 & -24.10 & 38.20 & 0.43633 \\
\hline 1.61 & 12.67 & 4.16 & 2.67 & 6.06 & 0.03497 & 118.42 & 102.62 & 142.66 & 0. & 9.84 & -2.87 & 35.23 & 053 \\
\hline 1.96 & 2.67 & 3.73 & 2.30 & 4.83 & 0.01361 & 115.19 & 99.30 & 132.73 & 0.18428 & 3.09 & -14.94 & 34.86 & 0.27806 \\
\hline 1.96 & 3.45 & 3.69 & 2.13 & 5.46 & 0.01077 & 116.14 & 98.63 & 140.90 & 893 & 2.68 & $\begin{array}{l}-17.89 \\
\end{array}$ & 37.42 & 3184 \\
\hline 1.96 & 4.22 & 3.71 & 2.39 & 5.18 & 166 & 117.91 & 103.06 & 143.16 & 19 & 5.70 & -17.99 & 39.21 & 260 \\
\hline 1.96 & 5.00 & 3.51 & 2.06 & 4.88 & 0.01199 & 113.84 & 96.45 & 141.15 & 0.16402 & 9.92 & -25.52 & 48.15 & 0.25030 \\
\hline 1.96 & 5.78 & 3.49 & 2.32 & 4.99 & 0.01183 & 110.03 & 95.35 & 129.39 & 0.16341 & 4.41 & -22.15 & 43.38 & 0.22989 \\
\hline 1.96 & 6.55 & 3.62 & 2.19 & 4.90 & 079 & 109.60 & 95.59 & 124.94 & 613 & 3.22 & -22.28 & 37.19 & 254 \\
\hline 1.96 & 7.33 & 3.63 & 2.35 & 4.62 & 0.01256 & 109.66 & 97.43 & 124.29 & 0.17358 & 2.88 & -18.60 & 29.52 & 0.24054 \\
\hline 1.96 & 12.67 & 3.76 & 2.50 & 4.95 & 0.01186 & 114.80 & 100.83 & 132.22 & 0.18871 & 9.77 & -12.84 & 27.78 & 0.23007 \\
\hline 1.96 & 13.45 & 3.77 & 1.96 & 5.06 & 0.01040 & 113.73 & 97.10 & 134.50 & 0.16062 & 9.06 & -12.43 & 29.52 & 0.20194 \\
\hline 1.96 & 14.22 & 3.80 & 2.46 & 5.35 & 0.01118 & 110.66 & 94.87 & 124.41 & 0.16296 & 9.73 & -15.34 & 34.20 & 0.19435 \\
\hline 1.96 & 15.00 & 3.47 & 2.21 & 4.87 & 0.01068 & 112.68 & 96.69 & 130.76 & 0.16646 & 5.67 & -21.77 & 32.35 & 0.23866 \\
\hline 1.96 & 15.78 & 3.50 & 2.35 & 4.79 & 0.01033 & 116.43 & 97.31 & 131.14 & 0.14422 & 6.98 & -20.88 & 31.09 & 0.25017 \\
\hline 1.96 & 16.55 & 3.43 & 2.17 & 4.76 & 0.01195 & 114.46 & 94.29 & 137.16 & 0.20962 & 14.24 & -18.40 & 45.06 & 0.25953 \\
\hline 1.96 & 17.33 & 3.51 & 2.41 & 4.70 & 0.01353 & 111.62 & 97.48 & 128.86 & 0.20370 & 15.03 & -14.82 & 35.84 & 0.29337 \\
\hline 6.41 & 2.67 & 2.73 & 1.52 & 3.91 & 0.00934 & 91.84 & 74.94 & 104.81 & 0.12120 & 4.96 & -16.43 & 41.89 & 0.20899 \\
\hline 6.41 & 3.45 & 2.69 & 1.07 & 3.94 & 0.01290 & 93.83 & 77.79 & 108.65 & 0.17515 & 3.10 & -24.42 & 54.56 & 0.29817 \\
\hline 6.41 & 4.22 & 2.76 & 1.34 & 3.89 & 0.01323 & 96.50 & 80.60 & 116.34 & 0.17808 & 6.66 & -18.16 & 38.84 & 0.31150 \\
\hline 6.41 & 5.00 & 2.52 & 1.24 & 4.11 & 0.01378 & 96.97 & 76.01 & 112.02 & 0.19590 & 11.90 & -10.70 & 49.85 & 0.31170 \\
\hline 6.41 & 5.78 & 2.57 & 1.18 & 4.08 & 0.01471 & 92.52 & 72.48 & 113.81 & 0.25386 & 6.21 & -26.03 & 40.11 & 0.31119 \\
\hline 6.41 & 6.55 & 2.90 & 1.56 & 4.07 & 0.01335 & 86.81 & 65.44 & 104.80 & 0.20218 & 8.76 & -19.89 & 46.33 & 0.24833 \\
\hline 6.41 & 7.33 & 2.98 & 1.70 & 4.05 & 0.00972 & 84.18 & 66.70 & 104.02 & 0.14817 & 8.26 & $\begin{array}{l}-14.11 \\
\end{array}$ & 32.63 & 0.18958 \\
\hline
\end{tabular}


Water Velocity Measurements on an ESBS at John Day Dam

\begin{tabular}{|c|c|c|c|c|c|c|c|c|c|c|c|c|}
\hline 6.41 & 12.67 & 2.99 & 1.81 & 4.08 & 0.00884 & 83.95 & 71.24 & 99.22 & 0.11466 & 12.15 & -6.72 & \begin{tabular}{|l|l|}
31.43 & 0.15554 \\
\end{tabular} \\
\hline 6.41 & 13.45 & 3.05 & 2.15 & 4.00 & 0.01323 & 81.64 & 68.05 & 98.51 & 0.17512 & 12.76 & -10.22 & \begin{tabular}{l|l}
34.58 & 0.22223 \\
\end{tabular} \\
\hline 6.41 & 14.22 & 3.08 & 2.07 & 4.22 & 0.01324 & 79.56 & 66.17 & 97.96 & 0.16251 & 15.34 & -6.80 & \begin{tabular}{l|l|}
33.69 & 0.23617 \\
\end{tabular} \\
\hline 6.41 & 15.00 & 2.45 & 1.21 & 3.79 & 0.01323 & 80.34 & 62.45 & 95.28 & 0.20735 & 11.87 & -13.90 & \begin{tabular}{l|l}
39.94 & 0.29516 \\
\end{tabular} \\
\hline 6.41 & 15.78 & 2.57 & 1.26 & 3.96 & 0.01256 & 81.50 & 59.18 & 112.09 & 0.19580 & 11.09 & -15.25 & \begin{tabular}{l|l|}
35.14 & 0.26514 \\
\end{tabular} \\
\hline 6.41 & 16.55 & 2.70 & 1.60 & 4.05 & 0.01431 & 77.19 & 55.52 & 94.27 & 0.20433 & 15.95 & -18.85 & \begin{tabular}{|l|l|}
50.13 & 0.29974 \\
\end{tabular} \\
\hline
\end{tabular}




\section{Appendix F}

\section{Turbulence Intensity Values at 138 MW Turbine Load}




\section{Appendix F}

\section{Turbulence Intensity Values at 138 MW Turbine Load}

The table in this appendix lists turbulence intensity values for $\mathrm{X}, \mathrm{Y}$, and Z-vectors and magnitude turbulence intensity for flow data collected at locations along the bar screen surface of the ESBS at 138 MW turbine load.

\begin{tabular}{|c|c|c|c|c|c|}
\hline \multicolumn{2}{|c|}{ Location (ft.) } & \multicolumn{4}{|c|}{ RMS Turbulence Intensity (ft/sec) } \\
\hline Vertical & Horizontal & X-Vector & Y-Vector & Z-Vector & Magnitude \\
\hline 0.03 & 2.67 & 0.66 & 0.68 & 0.43 & 0.45 \\
\hline 0.03 & 3.45 & 0.32 & 0.24 & 0.27 & 0.14 \\
\hline 0.03 & 4.22 & 0.26 & 0.27 & 0.31 & 0.26 \\
\hline 0.03 & 5.00 & 0.29 & 0.33 & 0.29 & 0.18 \\
\hline 0.03 & 5.78 & 0.33 & 0.20 & 0.23 & 0.25 \\
\hline 0.03 & 6.55 & 0.25 & 0.19 & 0.21 & 0.14 \\
\hline 0.03 & 7.33 & 0.23 & 0.19 & 0.24 & 0.13 \\
\hline 0.03 & 12.67 & 0.55 & 0.52 & 0.44 & 0.38 \\
\hline 0.03 & 13.45 & 0.26 & 0.23 & 0.29 & 0.17 \\
\hline 0.03 & 14.22 & 0.40 & 0.32 & 0.26 & 0.31 \\
\hline 0.03 & 15.00 & 0.23 & 0.26 & 0.37 & 0.28 \\
\hline 0.03 & 15.78 & 0.36 & 0.44 & 0.25 & 0.18 \\
\hline 0.03 & 16.55 & 0.35 & 0.30 & 0.30 & 0.20 \\
\hline 0.03 & 17.33 & 0.37 & 0.34 & 0.31 & 0.21 \\
\hline 0.34 & 2.67 & 0.60 & 0.58 & 0.41 & 0.46 \\
\hline 0.34 & 12.67 & 0.50 & 0.45 & 0.45 & 0.34 \\
\hline 0.66 & 2.67 & 0.56 & 0.53 & 0.40 & 0.41 \\
\hline 0.66 & 12.67 & 0.55 & 0.44 & 0.44 & 0.40 \\
\hline 0.98 & 2.67 & 0.53 & 0.53 & 0.47 & 0.39 \\
\hline 0.98 & 12.67 & 0.50 & 0.45 & 0.41 & 0.36 \\
\hline 1.29 & 2.67 & 0.63 & 0.55 & 0.47 & 0.48 \\
\hline 1.29 & 12.67 & 0.60 & 0.44 & 0.43 & 0.46 \\
\hline 1.61 & 2.67 & 0.65 & 0.51 & 1.00 & 0.92 \\
\hline 1.61 & 12.67 & 0.67 & 0.41 & 0.83 & 0.91 \\
\hline 1.96 & 2.67 & 0.36 & 0.43 & 0.29 & 0.33 \\
\hline 1.96 & 3.45 & 0.33 & 0.41 & 0.30 & 0.30 \\
\hline 1.96 & 4.22 & 0.40 & 0.42 & 0.27 & 0.35 \\
\hline 1.96 & 5.00 & 0.36 & 0.38 & 0.33 & 0.36 \\
\hline 1.96 & 5.78 & 0.36 & 0.35 & 0.31 & 0.34 \\
\hline 1.96 & 6.55 & 0.32 & 0.36 & 0.29 & 0.30 \\
\hline 1.96 & 7.33 & 0.35 & 0.37 & 0.31 & 0.32 \\
\hline 1.96 & 12.67 & 0.35 & 0.39 & 0.31 & 0.31 \\
\hline 1.96 & 13.45 & 0.35 & 0.38 & 0.32 & 0.32 \\
\hline 1.96 & 14.22 & 0.36 & 0.38 & 0.33 & 0.34 \\
\hline 1.96 & 15.00 & 0.35 & 0.43 & 0.31 & 0.33 \\
\hline 1.96 & 15.78 & 0.32 & 0.43 & 0.26 & 0.30 \\
\hline 1.96 & 16.55 & 0.42 & 0.47 & 0.37 & 0.38 \\
\hline 1.96 & 17.33 & 0.40 & 0.48 & 0.35 & 0.38 \\
\hline 6.41 & 2.67 & 0.33 & 0.34 & 0.23 & 0.32 \\
\hline 6.41 & 3.45 & 0.31 & 0.31 & 0.21 & 0.31 \\
\hline 6.41 & 4.22 & 0.35 & 0.36 & 0.22 & 0.33 \\
\hline 6.41 & 5.00 & 0.35 & 0.32 & 0.24 & 0.34 \\
\hline 6.41 & 5.78 & 0.37 & 0.36 & 0.32 & 0.37 \\
\hline 6.41 & 6.55 & 0.32 & 0.32 & 0.28 & 0.34 \\
\hline 6.41 & 7.33 & 0.32 & 0.35 & 0.30 & 0.33 \\
\hline
\end{tabular}


Water Velocity Measurements on an ESBS at John Day Dam

\begin{tabular}{|r|r|r|r|r|r|}
\hline 6.41 & 12.67 & 0.32 & 0.30 & 0.25 & 0.33 \\
\hline 6.41 & 13.45 & 0.33 & 0.31 & 0.27 & 0.35 \\
\hline 6.41 & 14.22 & 0.36 & 0.32 & 0.25 & 0.36 \\
\hline 6.41 & 15.00 & 0.34 & 0.33 & 0.26 & 0.34 \\
\hline 6.41 & 15.78 & 0.33 & 0.33 & 0.25 & 0.34 \\
\hline
\end{tabular}




\section{Distribution}

No. of

Copies

ONSITE

28 Pacific Northwest National Laboratory

T.J. Carlson (20) BPO

C.B. Cook K9-33

T.L. Gilbride $\quad$ BPO

R.P. Mueller k6-85

M.A. Weiland (5) BPO

\section{OFFSITE}

Richard Vaughn

U.S. Army Corps of Engineers, Portland District

Tony Norris

U.S. Army Corps of Engineers, Portland District

Matt Hanson

U.S. Army Corps of Engineers, Portland District

Robert Davidson

WES, Vicksburg, Mississippi

Don Kum (2)

John Day Dam 